\title{
HONEYDEW AS A NEWLY DESCRIBED ROUTE OF INSECTICIDE EXPOSURE TO BENEFICIAL INSECTS
}

Miguel Calvo Agudo 


\section{Propositions}

1. Including honeydew as a route of exposure will improve environmental risk assessments to evaluate pesticide safety.

(this thesis)

2. Banning of water-soluble systemic pesticides that are persistent in the environment will mitigate insect decline.

(this thesis)

3. The publication of studies with only statistically non-significant findings makes science more efficient.

4. Changing the definition of intelligence to a non-anthropocentric perspective will allow appreciation of all living organisms and their role on Earth.

5. Countries with an average meat consumption of more than $60 \mathrm{~kg}$ per capita per year contribute disproportionately to climate change.

6. Supporting diversity, inclusiveness and equality in environmental scientific institutions will help in the fight against climate change.

7. Providing children access to environmental education leads to the development of love and respect for nature.

Propositions belonging to the thesis entitled:

Honeydew as a newly described route of insecticide exposure to beneficial insects Miguel Calvo Agudo

Wageningen, 4 November 2021 


\section{Honeydew as a newly described route of insecticide exposure to beneficial insects}

Miguel Calvo Agudo 


\section{Thesis committee}

\section{Promotor}

Prof. Dr Marcel Dicke

Professor of Entomology

Wageningen University \& Research

\section{Co-promotor}

Dr Alejandro Tena

Centro de Protección Vegetal y Biotecnología, Instituto Valenciano de Investigaciones Agrarias Spain

\section{Other members}

Prof. dr Liesje Mommer, Wageningen University \& Research

Prof. dr Paul J. van den Brink, Wageningen University \& Research

Prof. dr Paul C. Struik, Wageningen University \& Research

Dr Jetske G. de Boer, Netherlands Institute of Ecology and Aeres University of Applied Sciences,

Wageningen

This research was conducted under the auspices of the graduate school Production Ecology \& Resource Conservation 


\title{
Honeydew as a newly described route of insecticide exposure to beneficial insects
}

\author{
Miguel Calvo Agudo
}

\section{Thesis}

submitted in fulfilment of the requirements for the degree of doctor at Wageningen University by the authority of the Rector Magnificus,

Prof. Dr A.P.J. Mol, in the presence of the

Thesis Committee appointed by the Academic Board to be defended in public

on Thursday 4 November 2021 at 11 a.m. in the Aula. 
Miguel Calvo Agudo

Honeydew as a newly described route of insecticide exposure to beneficial insects 220 pages.

$\mathrm{PhD}$ thesis, Wageningen University, Wageningen, the Netherlands (2021)

With references, with summary in English

ISBN: 978-94-6395-925-4

DOI : https://doi.org/10.18174/551068 
"There is still so much in the world worth fighting for. So much that is beautiful, so many wonderful people working to reverse the harm, to help alleviate the suffering. And so many young people dedicated to making this a better world. All conspiring to inspire us and to give us hope that it is not too late to turn things around, if we all do our part"

By Jane Goodall 



\section{Table of contents}

\section{Chapter I}

\section{General introduction}

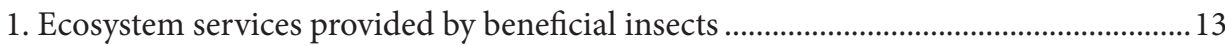

2. Plant-derived food sources for beneficial insects .............................................................. 15

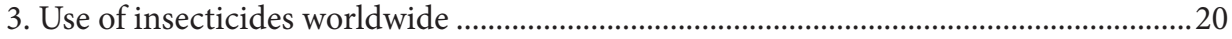

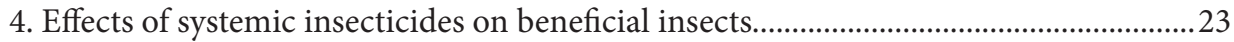

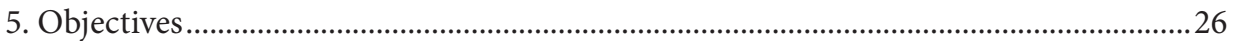

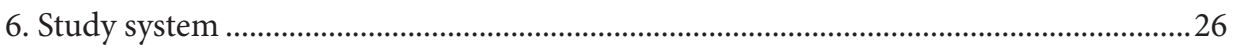

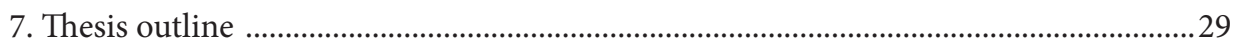

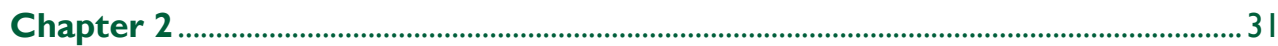

Neonicotinoids in excretion product of phloem-feeding insects kill beneficial insects

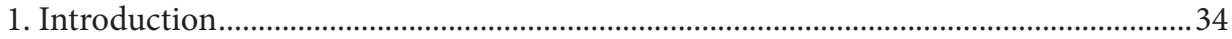

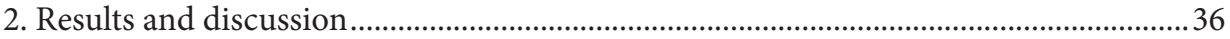

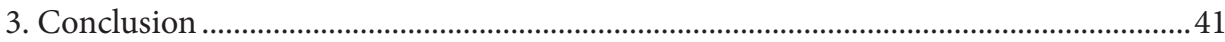

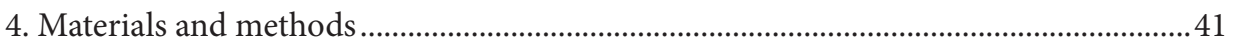

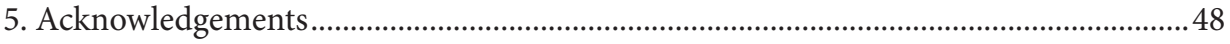

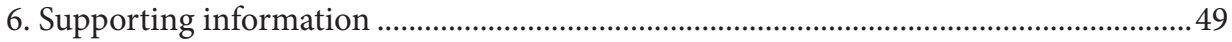

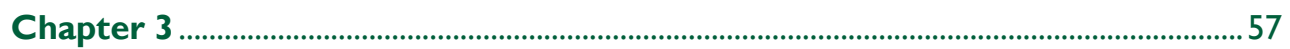

IPM-recommended insecticides harm beneficial insects through contaminated honeydew

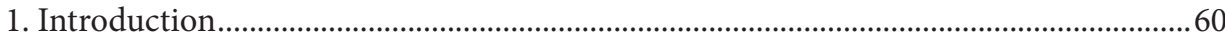

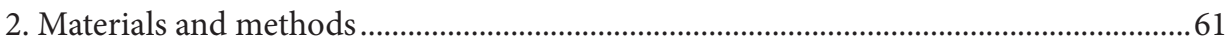

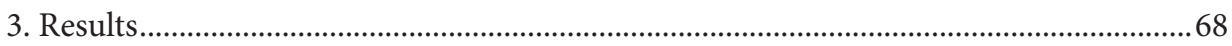

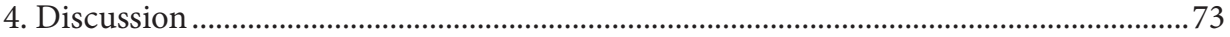

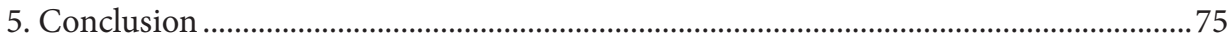

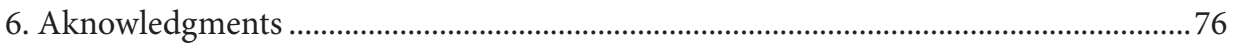

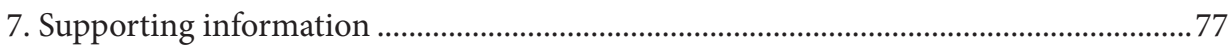




\section{Chapter 4}

Neonicotinoids from coated seeds toxic for honeydew-feeding biological control agents

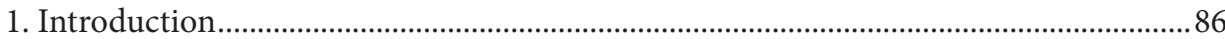

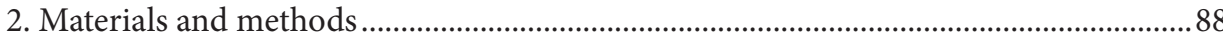

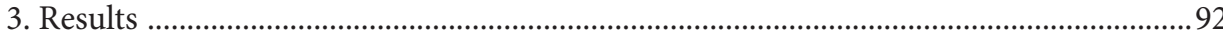

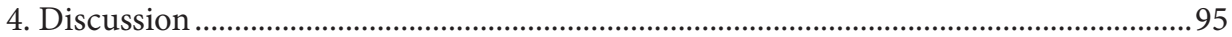

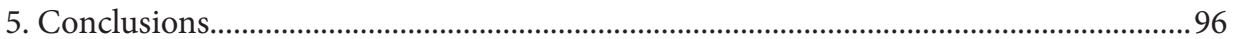

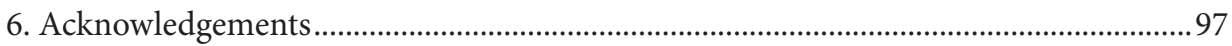

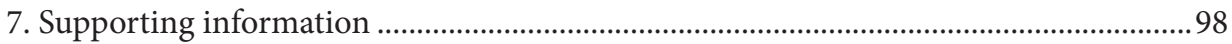

Chapter 5 ......................................................................................................... I 0 I

Beneficial insects do not avoid feeding on honeydew contaminated with neonicotinoids and may even prefer feeding on it

1. Introduction .104

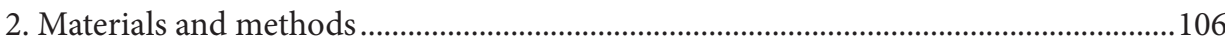

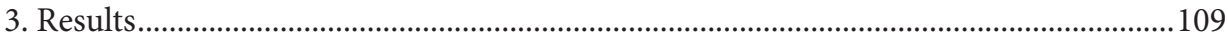

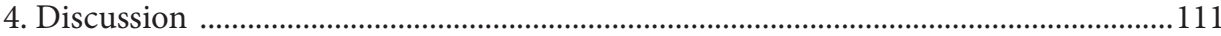

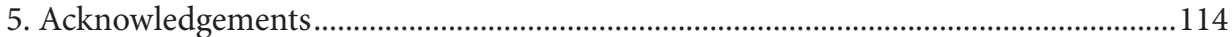

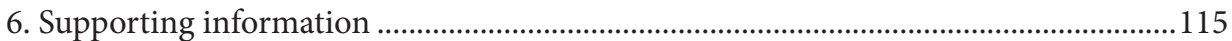

Chapter 6

Insecticide-contaminated honeydew: risks for beneficial insects in agriculture

1. Introduction 120

2. How can systemic insecticides reach honeydew?

3. What hemipteran species are more likely to excrete contaminated honeydew? .........124

4. What systemic insecticides are more likely to contaminate honeydew?

5. Potential crops in which honeydew can be contaminated with systemic insecticides

6. Conclusion

7. Supporting information

\section{Chapter 7}

\section{General discussion}


2. New frameworks for environmental risk assessments

3. Contaminated honeydew as a contributor to global insect decline .155

4. Concluding remarks on how this thesis contributes to science and society .158

References. 159

Summary 199

Acknowledgements .205

About the author .213

List of publications by Miguel Calvo-Agudo .221 



\section{Chapter I}

General introduction

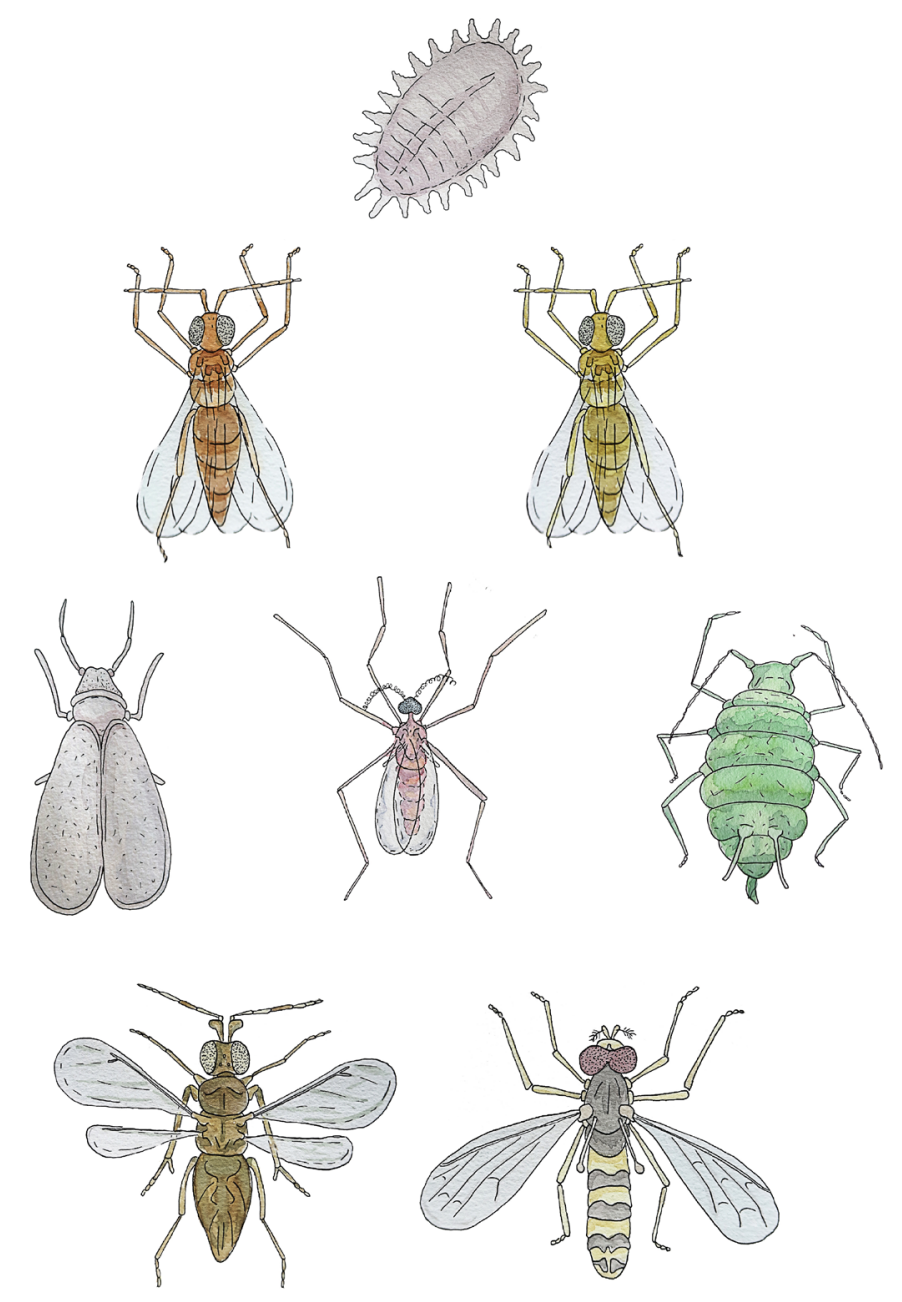





\section{ECOSYSTEM SERVICES PROVIDED BY BENEFICIAL INSECTS}

Beneficial insects provide vital ecosystem services including pollination, biological control of pests and weeds, nutrient cycling and providing food sources to higher trophic levels in the food web (Resh and Cardé, 2009). These ecosystem services are of such importance that their annual value has been estimated to be at least $\$ 57$ billion, only in the United States (Losey and Vaughan, 2006). Here, we will focus on two of the main groups of beneficial insects: biological control agents of agricultural pests and pollinators.

Biological control is the use of a population of one living organism to reduce the population of another organism (van Lenteren et al., 2017). Insects that are currently used in biological control comprise parasitic wasps and predators. Biological control can be subdivided in four categories. i) Natural biological control is an ecosystem service whereby pest populations are reduced by naturally occurring beneficial organisms. Humans do not interfere in this type of biological control. Nonetheless, this is the most valuable type of biological control to agriculture in economic terms, with an annual estimated value of $\$ 4.49$ billion, or $\$ 417$ per ha and year, only in the United States (Costanza et al., 1998; Losey and Vaughan, 2006; van Lenteren et al., 2017). ii) Conservation biological control consists of enhancing the performance of naturally occurring biological control agents by conserving their resources and reducing the pesticide-induced mortality of biological control agents (Holland et al., 2016; van Lenteren et al., 2017). iii) Classical biological control consists of the introduction, in an area where a pest is invasive, of a biological control agent collected from the area where the pest is native (van Lenteren et 
al., 2017). Classical biological control often results in enormous economic benefits due to a permanent pest population reduction (Cock et al., 2010; van Lenteren et al., 2017). For instance, the African citrus psyllid, Trioza erytreae (Del Guercio) (Hemiptera: Triozidae), is a vector of the citrus greening or huanglongbing (HLB) disease. This pest has recently invaded the Iberian Peninsula and Canary Islands. In order to reduce its population levels, a classical biological control program has been successfully implemented with the introduction of the South African parasitoid Tamarixia dryi (Waterson) (Hymenoptera: Eulophidae) (Urbaneja-Bernat et al., 2020a, 2019). iv) Augmentative biological control consists of mass-rearing and releasing large numbers of living organisms for pest control. In 2015, augmentative biological control was applied on more than 30 million ha worldwide with a market value of $\$ 1.7$ billion (van Lenteren et al., 2017).

Pollination is the process by which flowering plants reproduce and develop offspring (i.e. seeds). Insects, the main pollination vectors, are responsible of the reproduction of $87 \%$ of plants worldwide (Ollerton et al., 2011) and 35\% of the global crop-based food production (Klein et al., 2007). The global economic value of pollination from domesticated and wild animals has been estimated at $€ 153$ billion (Gallai et al., 2009). The honeybee Apis mellifera L. (Hymenoptera: Apidae) is the most versatile, ubiquitous and commonly used pollinator in agriculture. Recent research estimated that honeybees increased fruit set in only $14 \%$ of the systems surveyed and they are responsible for $39 \%$ of the visits to crop flowers (Garibaldi et al., 2013; Rader et al., 2015). However, the value of other wild pollinators, including non-bee species, is key to maintain yields and enhance fruit set in agriculture and sustain life on earth (Rader et al., 2015). Non-bee insects such as dipterans, lepidopterans, coleopterans or non-bee hymenopterans are also key pollinators with $38 \%$ of the visits to crop flowers. Among this non-bee pollination group, flies of the family Syrphidae (commonly known as hoverflies) are the most important pollinators.

Growing evidence of important declines in insect populations has caused great concern among the scientific community (Biesmeijer et al., 2006; Dirzo et al., 2014; Goulson et al., 2008; Hallmann et al., 2017; Harvey et al., 2020; Potts et al., 2010). Studies carried out in a rainforest of Puerto Rico (Lister and Garcia, 2018) and European protected areas (Hallmann et al., 2017) showed insect biomass losses of more than 75\% between 1989 and 2016. Most studies have pointed out that the main factors of insect decline are habitat degradation, scarcity of floral resources, global pollution with insecticides, fertilizers or herbicides, the increasing number 
of invasive species, and climate change. However, the relative importance of each factor that causes insect decline is still poorly understood. In addition, it is unknown whether insect decline has negative effects on biological control and pollination in agricultural lands. What is well known is that insecticides used to control pests have negative effects on non-target living organisms via many different routes with largely unknown effects (Frank and Tooker, 2020). This thesis aims to provide new evidence on how insecticides reach non-target beneficial organisms through plantderived food sources, which will further help research institutes and environmental protection agencies to carry out new studies that evaluate insect decline.

\section{PLANT-DERIVED FOOD SOURCES FOR BENEFICIAL INSECTS}

Many beneficial insect species, at least at some stage during their life cycle, feed on plantderived materials to meet their requirements for daily physical activities and metabolic processes (Heimpel and Jervis, 2005; Nicolson et al., 2007). They feed: i) directly upon plants, consuming fruits, nectar and extrafloral nectar, guttation, pollen, seeds, pearl bodies, sap, epidermis, or trichomes (Heimpel and Jervis, 2005; Singh et al., 2021; Urbaneja-Bernat et al., 2020b); and, indirectly, consuming honeydew excreted by several groups of herbivorous insects (Heimpel and Jervis, 2005).

The carbohydrates and other nutrients in minor proportion obtained from nectar and honeydew can enhance the longevity, fecundity (Berndt and Wratten, 2005), progeny fitness (Amorós-Jiménez et al., 2014; Lundgren, 2009), and proportion of female offspring (Berndt and Wratten, 2005; Heimpel and Lundgren, 2000) of beneficial insects. Moreover, they may also affect other biological traits such as diapause and quiescence (Michaud and Qureshi, 2006). Therefore, the scarcity or contamination of these plant-derived food sources in agricultural lands have negative impacts on pollination and biological control services provided by beneficial insects (Botías et al., 2015; Gurr et al., 2017; Rundlöf et al., 2015).

\section{I. Main plant-derived food sources for beneficial insects in agroecosystems}

In this thesis, I will focus on plant-derived food sources that are mainly composed of carbohydrates. In agriculture, beneficial insects find carbohydrates mainly in fruit, floral nectar, extrafloral nectar, guttation, or honeydew (Girolami et al., 2009; Heimpel and Jervis, 2005; Lundgren, 2009; Wäckers et al., 2008, 2005). These sources, however, have a highly diverse nutritional quality, and their availability depends on the crop and season (Figure 1). 


\begin{tabular}{|c|c|c|c|c|c|}
\hline & \multicolumn{5}{|c|}{ MAIN PLANT-DERIVED FOOD SOURCES FOR INSECTS IN AGRICULTURE } \\
\hline & $\Re$ & Л & ת & I & 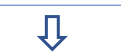 \\
\hline & HONEYDEW & NECTAR & EXTRAFLORAL NECTAR & GUTTATION & FRUIT \\
\hline $\begin{array}{l}\text { NUTRITIONAL } \\
\text { QUALITY }\end{array}$ & Variable & High & High & Variable & High \\
\hline $\begin{array}{l}\text { SEASONAL } \\
\text { AVAILABILITY }\end{array}$ & High & Low & High & $\begin{array}{l}\text { Depends on } \\
\text { weather and soil } \\
\text { conditions }\end{array}$ & Low \\
\hline CROPS & All crops & $\begin{array}{l}\text { All crops except cereals and } \\
\text { those harvested before } \\
\text { flowering (broccoli, } \\
\text { cauliflower, leafy greens, } \\
\text { carrots, leeks, etc.) }\end{array}$ & $\begin{array}{l}\text { Cotton, pumpkin, } \\
\text { zucchini, cassava, bean, } \\
\text { pea, almond, peach, } \\
\text { cherry, etc. }\end{array}$ & $\begin{array}{l}\text { Cereals, tomato, } \\
\text { cucumber, tobaco, } \\
\text { berries, cotton }\end{array}$ & $\begin{array}{l}\text { Vegetables, } \\
\text { fruits }\end{array}$ \\
\hline
\end{tabular}

Figure 1 | Summary of the main plant-derived food sources for beneficial insects in agriculture, their nutritional quality for insects and seasonal availability, the main crops in which they are present. Data obtained from: Girolami et al. (2009); Lundgren (2009); Tena et al. (2016); Urbaneja-Bernat et al. (2020); Wäckers et al. (2008).

\section{I.I. Fruits}

Fruits are a nutrient-rich food source for beneficial insects. However, fruits are scarce in nature and inaccessible for many beneficial insects because: i) fruits have physical barriers, such as skin, shells, waxes or trichomes that among other functions, obstructs herbivores from feeding on the fruit (Fernández et al., 2011); ii) fruits have low seasonal availability; iii) most fruits are harvested before they are mature enough to become accessible for beneficial insects; and iv) humans protect fruits against herbivorous pests by using covers or applying insecticides that make this nutritious food inaccessible or toxic for beneficial insects.

\section{I.2. Floral nectar}

Floral nectaries are structures used by the plant to accomplish pollination. For nectar producing plants, the amount of nectar produced per flower can vary from less than $1 \mu \mathrm{l}$ to a few $\mathrm{ml}$ (Nicolson et al., 2007). The primary nutrients in floral nectar are carbohydrates such as the sugars sucrose, glucose and fructose; although amino acids, lipids, enzymes antibiotics, antioxidants or toxic compounds can also be present in low quantities in some nectars (Lundgren, 2009; Nicolson et al., 2007). Generally, the nutritional quality of nectar is high, and numerous sugar-feeding beneficial insects feed on nectar when it is available. However, the mere presence of flowering plants is no guarantee of benefits to beneficial insects (Gurr et al., 2017). Indeed, many factors including floral architecture, flowering 
period, floral area or flower color might limit the value of floral resources to beneficial insects (Heimpel and Jervis, 2005). In most agroecosystems, floral nectar is scarce, because: i) several crops including cereals, tomato, kiwi, grape vine, or some ornamental flowers such as roses do not have nectar in their flowers, ii) numerous crops such as most Brassica species, leeks, onions, carrots, plants, leeks, chard, spinaches, artichokes, etc. are harvested before the flowering period, iii) nectar is limited to the brief flowering period of the crop and therefore, it is ephemeral (Gurr et al., 2017; Lundgren, 2009; Wäckers et al., 2008), iv) droughts resulting from hot weather reduce the abundance of floral units and the volume of nectar per plant (Phillips et al., 2018), and v) flowering nectar plants along crop borders, ditches, and roadsides in conventional agricultural landscapes are scarce or absent.

\section{I.3. Extrafloral nectar}

Extrafloral nectaries are sugar-secreting organs located in leaf laminae, petioles, rachids, bracts, stipules, pedicels, etc. that appear in numerous plant species worldwide (Lundgren, 2009; Rogers, 1985). The main role of extrafloral nectar is to attract plantprotecting biological control agents and provide them with food in exchange for their protection against herbivores (Choh and Takabayashi, 2006; Heil, 2015; Lundgren, 2009). Extrafloral nectar is mainly composed of mono- and di-saccharides, with glucose, fructose and sucrose being the dominants (Baker et al., 1978). As occurs with floral nectar, amino acids (Keeler, 1977), micronutrients and secondary chemicals are present in extrafloral nectar at much lower concentrations than carbohydrates. Lipids are seldom found (Caldwell and Gerhardt, 1986). Extrafloral nectar is available during most of the season and has a great nutritional quality for biological control agents (Stapel et al., 1997). However, most crops do not have extrafloral nectar. Indeed, only 300 plant species from more than 40 families have extrafloral nectaries (Rogers, 1985), and from those, few are crops (Figure 1).

\section{I.4. Guttation}

Plant guttation is composed of droplets secreted at the margins and tips of leaves through the hydathodes (pores). Guttation contains sugars and proteins at very variable concentrations (Goatley and Lewis, 1966; Grunwald et al., 2003; Komarnytsky et al., 2000; Singh and Singh, 2013; Urbaneja-Bernat et al., 2020b). This exudation is controlled by root pressure and is triggered by a combination of biotic (i.e. plant growth) and abiotic (i.e. ambient and soil temperatures, relative humidity and solar radiation, wind, soil moisture, soil nutrients, time of the day, etc.) factors that push up the plant assimilates through the xylem vessels and the apoplastic area (Grunwald et al., 2003; Singh, 2016). 
Guttation has been described in cereals, tobacco, tomato, berries or cucumber (Singh, 2016; Urbaneja-Bernat et al., 2020b) and is limited by climatic conditions that favor the exudations. For example, in nights where transpiration does not occur because stomata are closed and soil moisture level is high, roots take up water and create a hydrostatic pressure that forces guttation (Singh, 2016).

\subsubsection{Honeydew}

Honeydew is the excretion product of many hemipteran and few lepidopteran species that feed on the plant vessels. In general, honeydew excreted by hemipterans of the suborder Sternorrhyncha is rich in carbohydrates because they feed on the phloem, which transports the elaborated nutrients throughout the plant (Bollard, 1960). In fact, more than $80 \%$ of the honeydew dry weight can be composed of carbohydrates (Ewart and Metcalf, 1956) with highly diverse sugar profiles (Tena et al., 2018). Honeydew can contain a wide range of carbohydrates, that include those synthesized i) by the plant, such as fructose, glucose, sucrose, or maltose, and ii) by the honeydew producer, such as trehalose, erlose, melezitose, raffinose, stachyose, mannitol or sorbitol. (Tena et al., 2013b; van Neerbos et al., 2020). Sternorrhyncha honeydew can also contain amino acids, micronutrients, sterols, secondary plant metabolites and/or microorganisms (Leroy et al., 2011; Lundgren, 2009; Shaaban et al., 2020; Wäckers, 2000; Yao and Akimoto, 2001).

The Sternorrhyncha suborder comprises the superfamilies Phylloxeroidea [families: Adelgidae and Phylloxeridae], Aphidoidea [family Aphididae (aphids)] (Figure 2), Coccoidea [among other families: Coccidae (scales), Pseudococcidae (mealybugs), Dactylopiidae (cochineals) Margaroridae (ground pearls), Kerridae (lac scales)], Aleyrodoidea [family Aleyrodidae (whiteflies)] and Psylloidea [mainly families Psyllidae (psyllids) and Tryozidae (triozids)] (Figure 3).

Honeydew excreted by hemipterans from the suborder Auchenorrhyncha has no carbohydrates or only in very low concentrations, because they feed mostly on the xylem, although they sometimes feed on the phloem (Lundgren, 2009; Oya, 1980). This suborder includes the superfamilies Cercopoidea [family Cercopidae (froghoppers)], Membracoidea [families Cicadellidae (leafhoppers) and Membracidae (treehoppers)], Fulgoroidae (families Flatidae, Delphacidae, Hypochthonellidae, Meenoplidae, Tettigometridae, Cixiidae and Fulgoridae) (Figure 2). Similarly, lepidopterans from the families Lycaenidae and Riodinidae excrete honeydew in the larval stage through specialized glands, called Newcomer's glands, that are sometimes low in carbohydrates but may contain substantial quantities of amino acids (DeVries and Baker, 1989; Lundgren, 2009). 


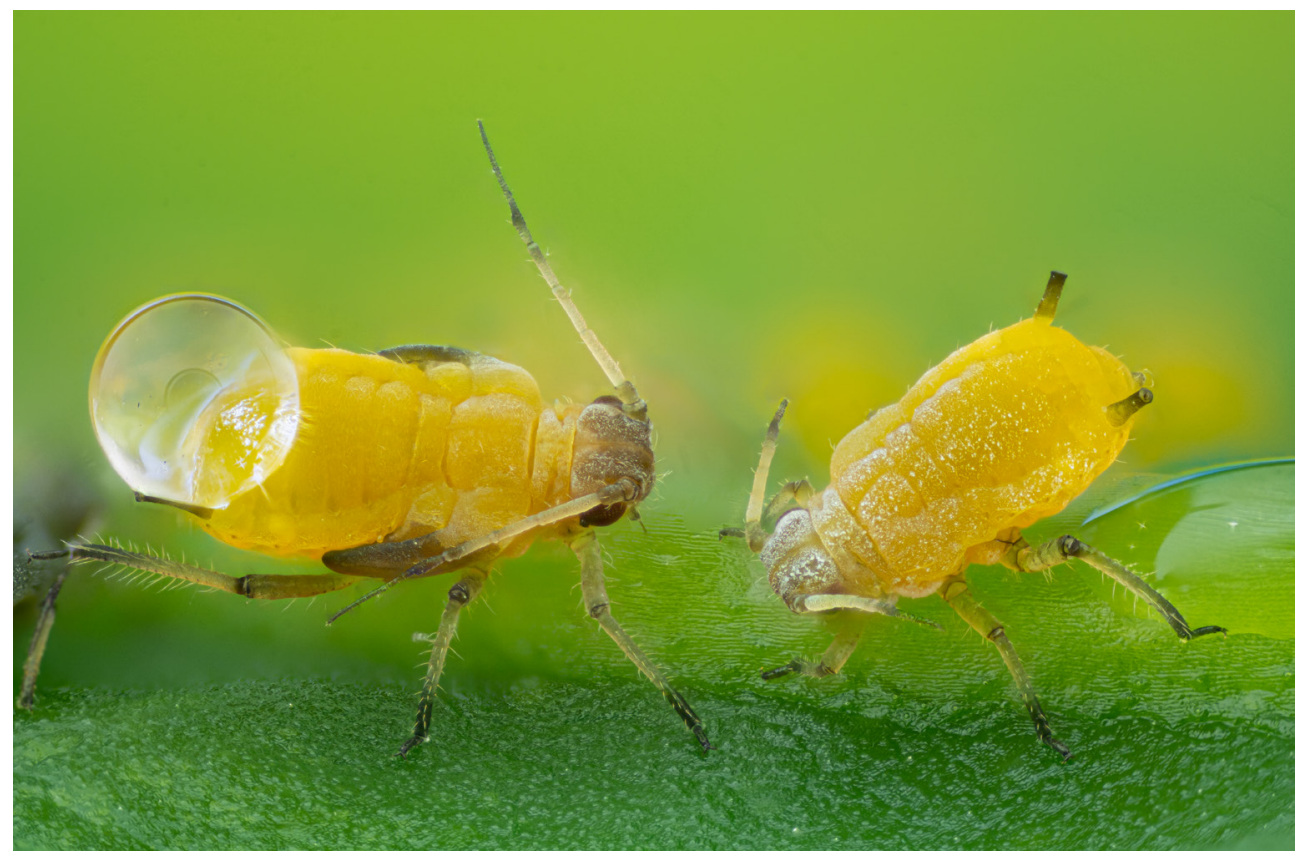

Figure 2 | Aphis sp. with a honeydew drop (Photo credits: Ángel Plata).

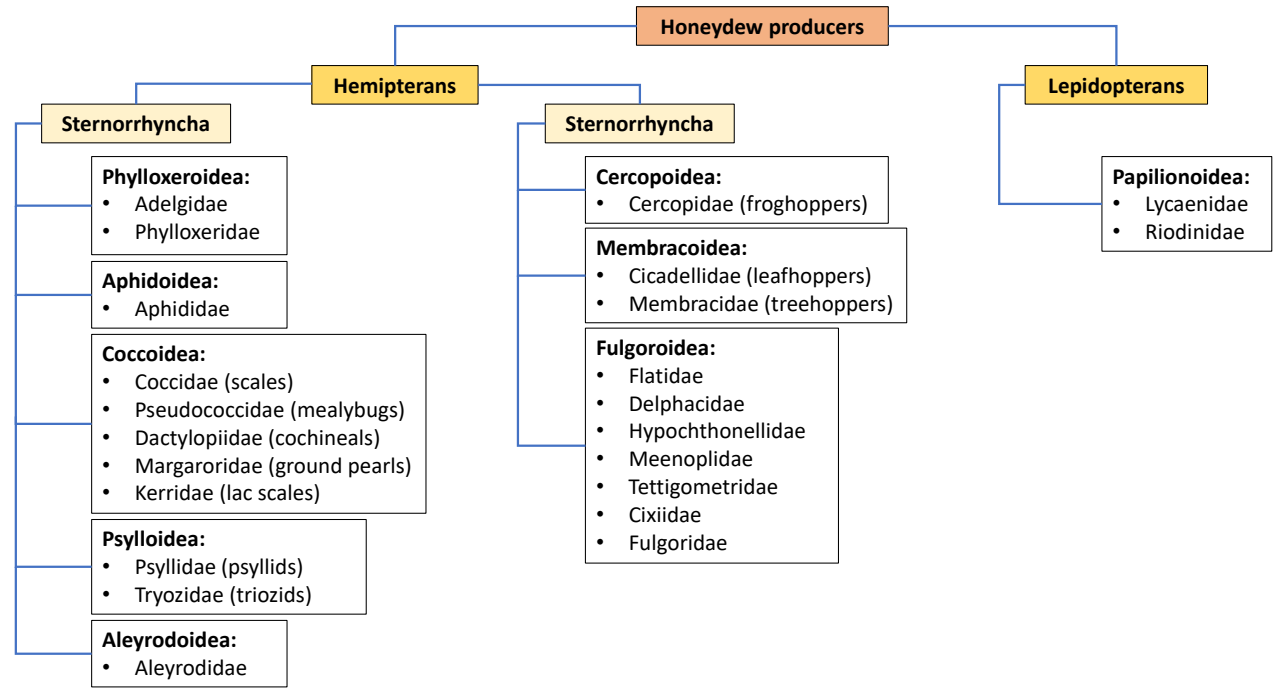

Figure 3 | Different honeydew producers across the class Insecta. From Moreno Ramírez (2020), with permission. 
Apart from the hemipteran species, the plant species, ant presence and hemipteran instar can also affect the chemical composition and nutritional value of honeydew for beneficial insects (Fischer and Shingleton, 2001; Tena et al., 2016; Yao and Akimoto, 2001). In some cases, the carbohydrate profile of honeydew is of such quality that it can extend the lifespan of honeydew-feeding biological control agents to the same extent as a highquality floral nectar (Rand and Waters, 2020). However, other honeydews do not extend the lifespan of honeydew-feeding biological control agents because their carbohydrate profile is poor (Tena et al., 2013a, 2013b).

Hemipterans feed on all crop species, making honeydew the most abundant and accessible carbohydrate source for beneficial insects that feed on plant-derived food sources in most agroecosystems. Unlike nectar, honeydew is present during most of the growing season (Tena et al., 2016; Wäckers et al., 2008). Indeed, this rich and ubiquitous food source is exploited by many insects with different feeding styles. For instance, many parasitic wasps, flies, ants, or predators rely on honeydew as a main carbohydrate source (Fratoni et al., 2019; Hogervorst et al., 2007; Lundgren, 2009; Rogers et al., 2007; Way, 1963). In addition, nectarivorous insects including bees, solitary bees, bumblebees, butterflies and moths will accept honeydew as food when encountered (Herrera, 1990; Konrad et al., 2009; Lundgren, 2009), especially during periods when nectar is scarce (Cameron et al., 2019; Meiners et al., 2017). In addition, some bee species are dependent on it as the main carbohydrate component of their diet (Dos Santos et al., 2019).

Honeydew is not only an important food source for beneficial insects. Many biological control agents such as parasitoids or predators use honeydew as an infochemical i.e. kairomone, to locate their hosts and prey (Budenberg, 1992, 1990; Ide et al., 2007). Interestingly, it has been found that host-associated bacteria emit specific volatiles that act as effective attractants for biological control agents to locate their host or prey (Fand et al., 2020; Leroy et al., 2011).

\section{USE OF INSECTICIDES WORLDWIDE}

Pesticides have undoubtedly contributed to a substantial increase in global food production (Silva et al., 2019). Indeed, the agricultural intensification that occurred between 1955 and 2000, which led to yield increases, came hand in hand with a $>750 \%$ increase in pesticide production (Guedes et al., 2016; Stehle and Schulz, 2015). Pesticides can be an important part of Integrated Pest Management (IPM) programs, when they are applied only as the last resort to prevent pest population outbreaks 
that cause economic damage (Tooker and Pearsons, 2021). However, pesticide use has shifted away from IPM programs towards preventative and prophylactic uses such as seed coatings or transgenic traits. As a consequence, pesticides are now applied in more crops and landscapes than at any time in history (Bernhardt et al., 2017; Stehle and Schulz, 2015; Tooker and Pearsons, 2021). Agricultural landscapes currently occupy ca. $40 \%$ of the world's total land surface (Foley et al., 2011; Stehle and Schulz, 2015). This intensification is linked directly with the use of pesticides, in which insecticides represent 7.5-10\% of the global pesticide use (Zhang, 2018).

\section{I. Physiochemical properties of insecticides}

The physiochemical properties of insecticides directly influence their solubility, uptake and mobility in the plant, which ultimately will affect the mode of application of each insecticide. These properties are: octanol/water-partition coefficient (log Kow), and the charge of their molecules at different $\mathrm{pH}$, which is measured with the acid dissociation constant (pKa) (Bromilow et al., 1990). These two properties are used to classify insecticides according to their mobility in the plant (Figure 4).

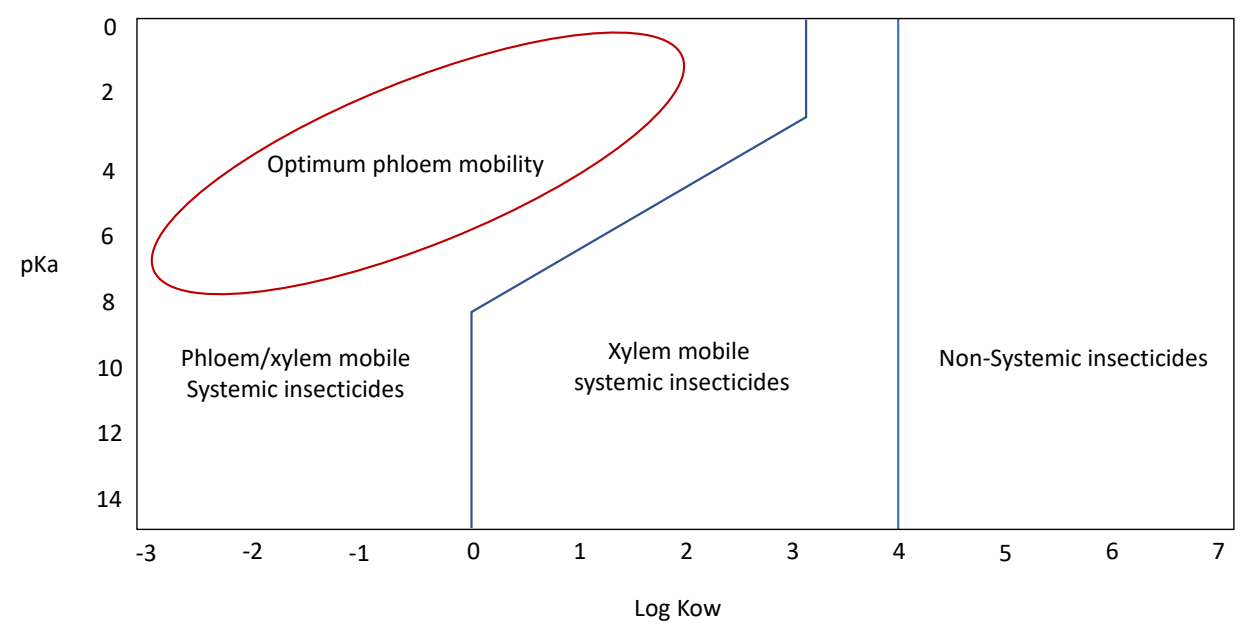

Hydrophilic

Lipophilic

Figure 4 | Expanded Bromilow model to predict the uptake and mobility of different active ingredients of plant protection products (Obtained from Bromilow and Chamberlain, 1991).

\section{I.I. Insecticide solubility and uptake}

Lipophilic active-ingredient molecules (log Kow values higher than 3 ) resist solubility in water (Teicher, 2017) (Figure 4). Therefore, to increase the solubility in water, lipophilic insecticides 
are typically mixed with other solvents or adjuvants. Furthermore, lipophilic insecticides are rapidly retained by the biological membranes of insect or leaf tissues and do not penetrate the biological membranes. These types of insecticides are classified as contact insecticides (Teicher, 2017) (Figure 4). Contact insecticides are usually sprayed onto the foliage.

Instead, hydrophilic insecticides solubilize easily in aqueous spray solutions, but they require the addition of surfactants in the mixture to facilitate the absorption across the lipophilic biological membranes (Figure 4). These insecticides are classified as systemic because once they are taken up by the plant they distribute to all plant tissues (Figure 4). Systemic insecticides tend to be hydrophilic to allow transportation and distribution within the plant (hydrophilicity: $\log \mathrm{K}_{\mathrm{ow}}<0$ ). However, having some non-aqueous solubility (lipophilicity: $\log \mathrm{K}_{\mathrm{ow}}>0$ ) can help the insecticide to permeate across biological membranes (Teicher, 2017).

\section{I.2. Mobility of systemic insecticides}

Systemic insecticides can be transported via phloem (symplastic transport) and/or xylem (apoplastic transport). The phloem mobility is bidirectional, downwards from existing leaves to the roots and upwards to new flushes, whereas xylem mobility is unidirectional, from roots to leaves.

Compared to contact insecticides, systemic insecticides were originally hailed as a perfect approach to IPM programmes because they could be selective to herbivorous insects that feed on treated plants. In this Thesis, I will focus on systemic insecticides, because their use has increased over the last decades and the security concerning beneficial insects should be re-evaluated (Krupke and Tooker, 2020).

\subsection{Main groups of systemic insecticides}

The Insecticide Resistant Action Committee (IRAC) classifies insecticides in different groups according to their mode of action. Systemic insecticides include many groups such as: carbamates, organophosphates, neonicotinoids; sulfoximines, flonicamid, pyridine azomethine derivatives, tetronic and tetramic acid derivatives, cyromazine, diacylhydrazines, phenyl-pyrazoles, methyl isothiocyanate generators, or diamides. This Thesis focusses on neonicotinoids because they are the most widely used insecticides worldwide, and on flonicamid and pymetrozine because their use is recommended in IPM programs.

Neonicotinoids are systemic insecticides that bind to the acetylcholine site on the nicotinic acetylcholine receptors (nAChRs), causing a range of symptoms from hyper- 
excitation to lethargy and paralysis (Insecticide Resistance Action Committee, 2020). These systemic insecticides are globally most widely used (Simon-Delso et al., 2014) because they can be applied against a broad range of insect pests in most crops and ornamentals. Neonicotinoids are highly persistent, as they can remain for more than one year in plant tissues (Byrne et al., 2014), and for more than ten years in the environment (Humann-Guilleminot et al., 2019). Due to their persistence and negative effects on nontarget beneficial insects (Pisa et al., 2015), the European Union banned the neonicotinoids imidacloprid, thiamethoxam and clothianidin for outdoor uses in 2019. This group of insecticides is, however, still allowed in most countries.

Flonicamid and pymetrozine, are newer classes of insecticides than carbamates, organophosphates and neonicotinoids. These insecticides are increasingly being used in agriculture because they are selective to insect pests or/and are less persistent in the environment than neonicotinoids (Harrewijn and Kayser, 1997; Liu et al., 2014; Morita et al., 2007; Shen et al., 2009). Flonicamid and pymetrozine are applied against numerous pests such as whiteflies, aphids, planthoppers or leafhoppers (Belchim, 2020; Syngenta, 2020). The metabolites of flonicamid and pymetrozine can remain in citrus for more than 60 and 21 days respectively, after their application (Belchim, 2020; Syngenta, 2020).

\section{EFFECTS OF SYSTEMIC INSECTICIDES ON BENEFICIAL INSECTS}

Insecticides are produced to control insect pests but they can also kill beneficial insects. In the last 30 years, the effects of insecticides on beneficial arthropods have been the subject of a growing number of studies, and the potential negative effects have been reviewed numerous times (Desneux et al., 2007).

\section{I. Lethal and sublethal effects of insecticides on beneficial insects}

Generally, the median lethal dose $\left(\mathrm{LD}_{50}\right)$ or lethal concentration $\left(\mathrm{LC}_{50}\right)$ are used to assess and compare the direct mortality caused by insecticides after the exposure to insecticides (Desneux et al., 2007). Furthermore, those insecticides that do not induce apparent mortality in beneficial insects, can cause physiological and behavioral effects on individuals that survive the exposure to a pesticide. For instance, a plethora of sublethal effects on the insect physiology such as an increase of offspring mortality, a reduction of longevity, fecundity or fertility of adults, or an increase in male-to-female sex ratio may occur. Furthermore, effects on the mobility, navigation and orientation, feeding behavior, oviposition behavior, and learning performance often occur. Some of these detrimental effects are summarized by Desneux et al. (2007). 


\subsection{Routes of exposure to systemic insecticides for beneficial insects}

In agro-ecosystems, beneficial insects can be exposed to systemic insecticides through several routes of exposure.

\subsection{Direct contact}

The most obvious and well-known exposure is when insecticides reach beneficial insects during their application. Insecticides can arrive via direct exposure of the insecticide, or via droplets and dust particles derived from drift (Girolami et al., 2012; Martinou et al., 2014; Nuyttens et al., 2013; Planes et al., 2013; Sgolastra et al., 2012; Thompson, 2001). For example, drift of insecticide-containing particles released during the sowing of seeds coated with systemic insecticides can reach beneficial insects and some spring bee losses have been attributed to this drift in corn fields (Girolami et al., 2012). Moreover, beneficial insects can be harmed when they move on surfaces treated with insecticides (i.e. plant surface, soil and/or water) (Krupke et al., 2012; Martinou et al., 2014). For instance, some pollinator species are exposed to insecticides and harmed while nesting in contaminated soils or when using contaminated mud to build their nests (Anderson and Harmon-Threatt, 2019).

\subsubsection{Feeding on treated prey and hosts}

Biological control agents might be harmed when parasitic wasp larvae feed on their contaminated hosts (Taylor et al., 2015), or when predators consume contaminated prey (Yao et al., 2015). For instance, adults of the parasitic wasp Toxoneuron nigriceps (Viereck) (Hymenoptera: Braconidae) parasitize fewer hosts and live shorter when they have developed inside noctuid hosts fed on tobacco plants treated with imidacloprid than when they have developed inside noctuid hosts fed on untreated tobacco plants (Taylor et al., 2015). Also, larvae of the predator Cryptolaemus montrouzieri Mulsant (Coleoptera: Coccinellidae) do not reach the adult stage when they feed on pyriproxyfen-treated prey (Planes et al., 2013).

\subsubsection{Feeding on plant tissue}

Zoophytophagy is a type of omnivorous behavior that occurs when plant tissues are consumed by primarily predaceous species to increase the fecundity, and to reduce developmental time and cannibalism (Moser and Obrycki, 2009). For instance, zoophytophagous biological control agents such as the mirids Macrolophus pygmaeus (Rambur) and Nesidiocoris tenuis Reuter (Hemiptera: Miridae), the coccinellids Coleomegilla maculate De Geer and Harmonia axarydis Pallas (Coleoptera: Coccinellidae), and the phytoseiid mites Euseius stipulatus (Athias-Henriot) and Typhlodromalus aripo 
De Leon (Mesostigmata: Phytoseiidae) can be harmed while feeding on plant tissue that contains insecticides (Martinou et al., 2014; Moser and Obrycki, 2009).

\subsubsection{Feeding on plant-derived food sources}

Systemic insecticides can reach plant-derived food sources such as fruits, nectar, extrafloral nectar, guttation or pollen, which are some of the main food sources for pollinators and biological control agents (Azpiazu et al., 2019; Dively and Kamel, 2012; Girolami et al., 2009; Jones et al., 2020; Stoner and Eitzer, 2012). The translocation of systemic insecticides to plant-derived food has been considered one of the main routes of exposure to beneficial insects (Kyriakopoulou et al., 2017). While reaching plant-derived food sources, insecticides can cause lethal and sublethal effects on biological control agents (Krischik et al., 2007; Rogers et al., 2007; Stapel et al., 2000) and pollinators (Azpiazu et al., 2019; Whitehorn et al., 2012). Furthermore, pollinators prefer food contaminated with neonicotinoids when they have had prior access to contaminated food sources (Arce et al., 2018; Kessler et al., 2015).

One of the most important modes of application of systemic insecticides is as seed coating (seed treatment). This is the leading delivery method of some insecticides such as neonicotinoids (Frank and Tooker, 2020; Matsuda et al., 2020). For example, the seeds of over $50 \%$ of soybeans, $52-77 \%$ of cotton, and $79-100 \%$ of maize sown in the United States were coated with neonicotinoids in 2011 (Douglas and Tooker, 2015; Hurley and Mitchell, 2017). When a plant grows from a coated seed, systemic insecticides distribute to all tissues including floral and extrafloral nectar, and insects that feed on these food sources become exposed. Due to the negative impact on pollinators, this route of exposure has been highlighted as one of the main causes of pollinator declines (Rundlöf et al., 2015; Woodcock et al., 2017, 2016). For this reason, the use of three neonicotinoids (imidacloprid, thiamethoxam and clothianidin) was banned in the European Union for outdoor uses (European Food Safety Authority, 2018, 2013a).

\subsubsection{Honeydew}

As plant-derived source, honeydew can contain plant secondary compounds that are excreted by honeydew producers (Züst and Agrawal, 2015). However, it has never been investigated whether honeydew excreted by hemipterans feeding on insecticidetreated plants contain insecticides and becomes toxic to those arthropods that feed on it. This would represent a novel route of insecticide exposure for arthropods. This route of exposure could arise when honeydew producers survive insecticide treatments and excrete honeydew contaminated with insecticides. Such a route of insecticide exposure 
to beneficial insects could be very important because, unlike nectar, honeydew is highly abundant and accessible in most agroecosystems.

\section{OBJECTIVES}

Honeydew is the most abundant and accessible sugar source for beneficial insects in many agroecosystems. Despite its importance, this ubiquitous food source has been neglected as a potential source of insecticide exposure to beneficial insects. Therefore, the aim of this Thesis was to explore honeydew as a route of insecticide exposure for beneficial insects addressing several questions:

- Goal II. Is honeydew a route of exposure to systemic insecticides (neonicotinoids and feed deterrents) for beneficial insects? (chapter 2 for neonicotinoid insecticides and chapter 3 for feeding deterrent insecticides)

- Goal 2. Do neonicotinoids from coated seeds reach honeydew excreted by aphids and if so, are they toxic to beneficial insects? (chapter 4)

- Goal 3. Can beneficial insects discriminate between honeydew contaminated with neonicotinoids and uncontaminated honeydew? (chapter 5)

- Goal 4. What are the potential pathways of honeydew contamination? Which hemipteran families are more likely to excrete contaminated honeydew? Which insecticides are more likely to reach honeydew due to their physiochemical properties? Are there crops in which honeydew is more likely to be contaminated? (chapter 6)

\section{STUDY SYSTEM}

\section{I. Plant}

For goals 1 and 3, I selected citrus as crop because it is one of the most important crops in Spain and one of the main fruit crops cultivated globally. Citrus crops are being grown in $17.45 \%$ of the 55.4 Mhas that are used globally to grow citrus crops and other fruit crops such as: apple, grape, mango, banana, plum, peach, nectarine, apricot, persimmon, kiwi, melon, watermelon, strawberry or raspberry (FAOSTAT, 2021). Citrus crops harbour a diverse and dynamic complex of hemipterans that excrete honeydew during most of the growing season (Pekas et al., 2011; Tena et al., 2013a)

For goal 2, we used soybean because it is an herbaceous crop, typically grown in monocultures, representing $8.67 \%$ of the worldwide agricultural area (FAOSTAT, 2021). 


\subsection{Honeydew producer}

For goals 1 and 3, I used the mealybug Planococcus citri (Risso) (Hemiptera: Pseudococcidae) because it is a common pest in citrus-producing areas of the world (Urbaneja et al., 2020), whose honeydew increases the longevity and fecundity of beneficial insects (Tena et al., 2013a).

For goal 2, I used the soybean aphid Aphis glycines Matsumura (Hemiptera: Aphididae) because it is a common pest of soybean crops. In the USA, where it is an invasive species, it is a dominant pest that can reduce yields by 2.4 billion dollars annually if left untreated (Tilmon et al., 2011). Aphis glycines honeydew increases longevity of parasitic wasps (Tena et al., 2018; Wyckhuys et al., 2008).

\subsection{Systemic insecticides}

I selected four systemic insecticides with three different modes of action.

For goals 1, 2 and 3, I selected neonicotinoid insecticides because they are the most widely used insecticides against hemipteran pests (Frank and Tooker, 2020; Jeschke et al., 2011). The neonicotinoids used were thiamethoxam and imidacloprid because they use different translocation systems: via phloem and xylem, respectively (Jeschke et al., 2011). Neonicotinoids were applied using the three most common modes of application: soil drench and foliar spray in goal 1 and coated on seeds in goal 2 (Jeschke et al., 2011).

For goal 1, we also selected two phloem-transported feeding deterrent insecticides: pymetrozine and flonicamid. These insecticides are applied against aphids and whiteflies but mealybugs, which are concurrently infesting plants, are tolerant/resistant to these insecticides. Therefore, mealybugs may excrete honeydew with insecticide at high concentrations for long periods.

\subsection{Beneficial insects}

For goals 1 and 3, I selected two beneficial insects to test whether honeydew contaminated with insecticides is toxic for them. First, we used the hoverfly Sphaerophoria rueppellii (Wiedemann) (Diptera: Syrphidae) (Figure 5), because it i) is a pollinator (as adult) and a predator (during larval stages) (Pekas et al., 2020), ii) is commercially available, and iii) hoverflies are sensitive to insecticides (Vogel, 2017). Second, we selected the parasitic wasp Anagyrus vladimiri (Girault) (Hymenoptera: Encyrtidae) because: i) it is the main parasitic wasp of $P$. citri, ii) is commercially available, and iii) this species has been used to 
test whether neonicotinoids can reach parasitic wasps through extrafloral nectar (Krischik et al., 2007). Both beneficial insects are expected to feed on honeydew excreted by $P$. citri.

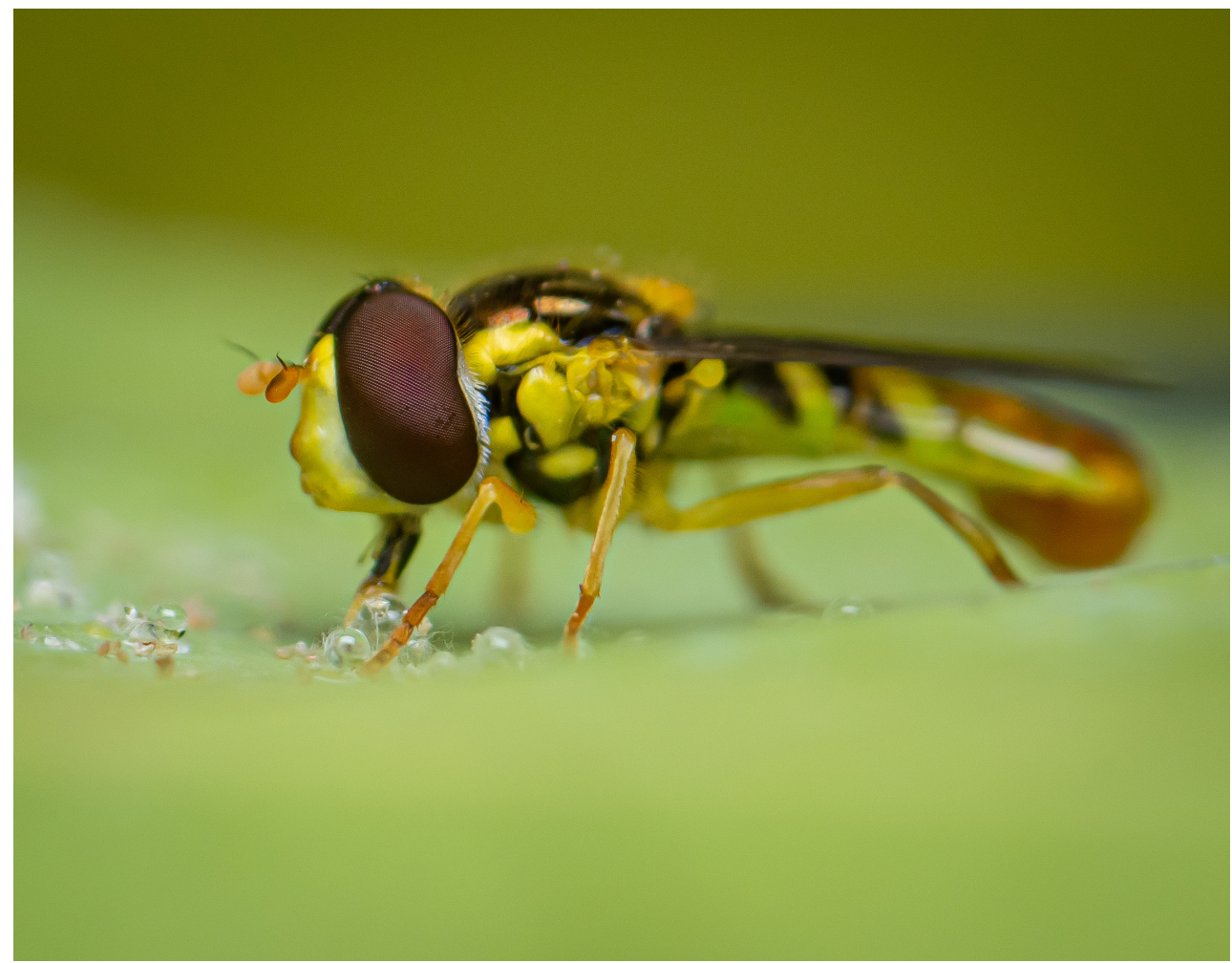

Figure 5 | The hoverfly Sphaerophoria rueppellii feeding on honeydew (Photo credits: Ángel Plata).

For goal 2, we selected the aphid-feeding predatory midge Aphidoletes aphidimyza (Rondani) (Diptera: Cecidomyiidae) because: i) it uses honeydew as food source and kairomone (Choi et al., 2004; Watanabe et al., 2014); and ii) is the most abundant dipteran predator in soybean fields from north central USA (Kaiser et al., 2007). We also selected the soybean aphid parasitic wasps Aphelinus glycinis Hopper and Woolley (Hymenoptera: Aphelinidae) and Aphelinus certus Yasnosh (Hymenoptera: Aphelinidae) because they are two of the main parasitic wasps of the soybean aphid A. glycines in North America. The former parasitic wasp was purposefully introduced in United States to control the soybean aphid (Hopper et al., 2017), whereas the latter parasitic wasp was accidentally introduced and is now abundant throughout soybean growing areas of North America (Frewin et al., 2010; Kaser and Heimpel, 2018; Miksanek, 2020; Miksanek and Heimpel, 2019). 


\section{THESIS OUTLINE}

Chapter 2 addresses honeydew as a newly discovered route of insecticide exposure to beneficial insects. For this, I first quantified the neonicotinoid concentration in the hemipteran honeydew after a foliar- or soil-application of insecticides. Afterwards, I carried out longevity bioassays to understand the toxicity of honeydew from mealybugs feeding on trees treated with either thiamethoxam or imidacloprid, on two beneficial insects: the parasitic wasp A. vladimiri and the hoverfly S. rueppellii.

Chapter 3 investigates whether apart from neonicotinoids, also systemic insecticides from other groups can reach honeydew. For this, I carried out similar investigations as those of chapter 2 but with the feeding-deterrent insecticides flonicamid and pymetrozine. These insecticides are commonly used in IPM programs. First, I quantified the concentration of flonicamid and pymetrozine in hemipteran honeydew. Second, I studied the toxicity on beneficial insects by feeding S. rueppellii and A. vladimiri with honeydew excreted by hemipterans feeding on trees treated with either pymetrozine or flonicamid. I collected hemipteran honeydew in field conditions and analysed it to study whether this route occurs in field conditions.

The research presented in Chapter 4 was conducted in Minnesota, one of the world's largest regions of soybean production. Here, I studied whether neonicotinoids from coated seeds reach honeydew excreted by the soybean aphid A. glycines in a soybean crop under field conditions. For this study, I infested soybean plants with aphids and collected their honeydew one month after sowing the plants in the field. This honeydew was analysed for neonicotinoid presence. Moreover, I offered aphid honeydew collected 35-36 days after sowing the soybean plants to the predatory midge A. aphidimyza and the parasitic wasps A. certus and A. glycinis to estimate the toxicity of contaminated honeydew.

Chapter 5 tests whether beneficial insects can discriminate between honeydew contaminated with neonicotinoids or uncontaminated honeydew. For this, I examined in choice tests whether the parasitic wasp A. vladimiri and the hoverfly S. rueppellii could discriminate between honeydew excreted by mealybugs feeding on plants treated with imidacloprid or thiamethoxam (contaminated honeydew) and honeydew excreted by mealybugs feeding on untreated plants (uncontaminated honeydew). This study was carried out for beneficial insects with and without previous feeding experience. 
Chapter 6 explores the potential pathways in which honeydew might be contaminated with insecticides. In detail, this perspective review hypothesizes: i) which hemipteran families are more likely to excrete contaminated honeydew and ii) which systemic insecticides are more likely to contaminate honeydew. Finally, we analyse several model crops in Europe and/or the USA where contaminated honeydew could be problematic for beneficial organisms that feed on this ubiquitous carbohydrate source.

Chapter 7 discusses the main contributions of my $\mathrm{PhD}$ to the field of insect ecotoxicology and connects the results of each chapter aiming to highlight the main ecological implications of this route of exposure. Furthermore, I explain how this thesis contributes to science and society. 


\section{Chapter 2}

\section{Neonicotinoids in excretion product of phloem-feeding insects kill beneficial insects}

Miguel Calvo-Agudo, Joel González-Cabrera, Yolanda Picó,

Pau Calatayud-Vernich, Alberto Urbaneja, Marcel Dicke, \& Alejandro Tena

Published in Proceedings of the National Academy of Sciences of the U.S.A.

https://doi.org/10.1073/pnas.1904298116
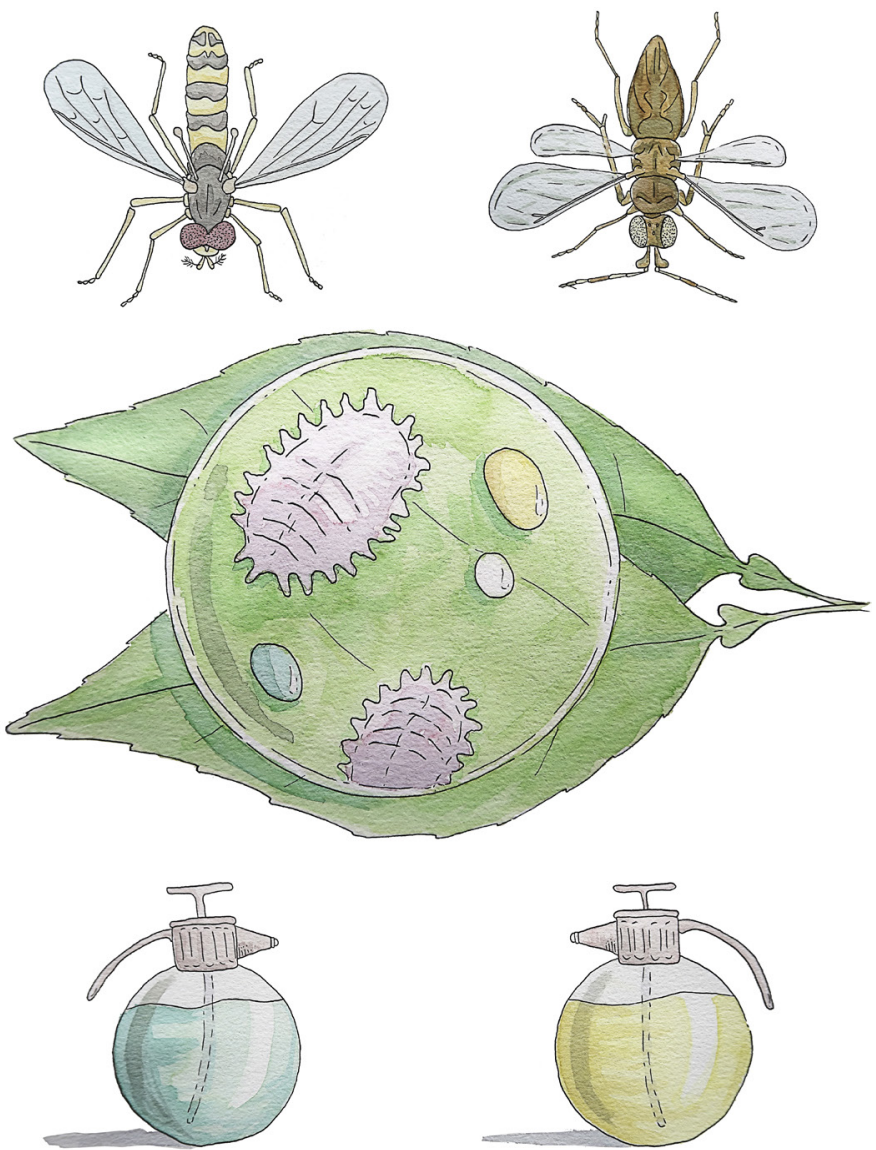



\section{ABSTRACT}

Pest control in agriculture is mainly based on the application of insecticides, which may impact non-target beneficial organisms leading to undesirable ecological effects. Neonicotinoids are among the most widely used insecticides. However, they have important negative side effects, especially for pollinators and other beneficial insects feeding on nectar. Here, we identify a new, more accessible exposure route: neonicotinoids reach and kill beneficial insects that feed on the most abundant carbohydrate source for insects in agroecosystems, honeydew. Honeydew is the excretion product of phloem-feeding hemipteran insects such as aphids, mealybugs, whiteflies and psyllids. We allowed parasitic wasps and pollinating hoverflies to feed on honeydew from hemipterans feeding on trees treated with thiamethoxam or imidacloprid, the most commonly used neonicotinoids. LC-MS/MS analyses demonstrated that both neonicotinoids were present in honeydew. Honeydew with thiamethoxam was highly toxic to both species of beneficial insects, and honeydew with imidacloprid was moderately toxic to hoverflies. Collectively, our data provide strong evidence for honeydew as a novel route of insecticide exposure that may cause acute or chronic deleterious effects on non-target organisms. This novel route should be considered in future environmental risk assessments of neonicotinoid applications. 


\section{INTRODUCTION}

Growing evidence of important declines in insect populations has caused great concern because of the valuable ecosystem services that insects provide, such as pollination, biological control, nutrient cycling, and providing food sources to higher trophic levels in the food web (Dirzo et al., 2014; Goulson et al., 2008; Hallmann et al., 2017; Ollerton et al., 2014; Potts et al., 2010; Sánchez-Bayo and Wyckhuys, 2019; Thomas et al., 2004). Some of the suggested causes for the decline in insect populations are the loss of their natural habitat, climate change and the widespread use of insecticides (Goulson et al., 2008; Hallmann et al., 2017; Ollerton et al., 2014; Potts et al., 2010; Sánchez-Bayo and Wyckhuys, 2019). Insecticide applications usually result in rapid mortality of the target herbivore species. However, insecticides can also affect beneficial insects directly, as well as indirectly through the food chain (Desneux et al., 2007; Stapel et al., 2000). Neonicotinoids are among the most widely used and toxic insecticides, accounting for more than $20 \%$ of the world's insecticide market (Jeschke et al., 2011). In 2012, they were used in important crops such as citrus, cotton, oilseed rape, soybean, ornamentals, fruits, greenhouse vegetables, potato, rice, sunflower seed, or maize (European Food Safety Authority, 2018). In that year, imidacloprid and thiamethoxam accounted for the largest share of authorized insecticide use in Europe, with 30 and 25\%, respectively (European Food Safety Authority, 2018). In Europe, 70\% of the neonicotinoid treatments were sprays, whereas less than $20 \%$ were seed treatments, and the rest were other application methods such as drip irrigation (European Food Safety Authority, 2018). In 2014, 33\% of the 239,000 ha dedicated to citrus production in California (USA) (Douglas and Tooker, 2015; Food Agriculture Organization of the United Nations, 2013) was treated with soil or foliar applications of imidacloprid and this insecticide remained in trees for more than one year (Byrne et al., 2014). These neonicotinoid-treated trees can be infested by various species of phloem-feeding insects that survive the treatment and excrete honeydew (Grafton-Cardwell, 1996; Grafton-Cardwell et al., 2013).

In contrast to previous generations of insecticides, neonicotinoids act systemically throughout the plant. Their use is questioned because of the impact on beneficial insects, mainly bees (Goulson et al., 2008; Whitehorn et al., 2012). One of the best-known routes of exposure of beneficial insects to neonicotinoids is through contaminated floral nectar and pollen (Stapel et al., 2000; Whitehorn et al., 2012). Neonicotinoids reach these plantderived food sources at concentrations ranging from 0.7 to $39 \mu \mathrm{g} / \mathrm{kg}$ (Byrne et al., 2014; Kessler et al., 2015; Whitehorn et al., 2012). Many insects are exposed to neonicotinoids 
when they feed on nectar and pollen during the flowering period of crops. However, floral nectar and pollen are scarce and limited to only the brief flowering period in many agroecosystems (Lundgren, 2009; Wäckers et al., 2008).

Honeydew is the most important source of carbohydrates in many ecosystems, especially in agricultural fields (Hogervorst et al., 2008; Lundgren, 2009; Tena et al., 2016; Wäckers et al., 2008). Honeydew is the sugar-rich excretion of phloem-feeding insects such as aphids, whiteflies, mealybugs, coccids, and psyllids that feed on crops, weeds, or the surrounding vegetation. This rich and ubiquitous food source is exploited by many beneficial insects, including bees, ants, parasitic wasps and predators (Lundgren, 2009; Tena et al., 2016), increasing their fitness by feeding on honeydew (Lundgren, 2009; Tena et al., 2018, 2016, 2013a; Wäckers et al., 2008). For instance, a great number of ant species, which protect honeydew producers, feed on honeydew and would not survive without it (Tena et al., 2016). Similarly, more than $50 \%$ of the naturally occurring parasitic wasps collected in wheat fields and citrus orchards had recently fed on honeydew (Hogervorst et al., 2007; Tena et al., 2013b). Most of these parasitic wasps would die in less than two days without feeding on honeydew (Wäckers et al., 2008). Bees, as well as other pollinators, also feed on honeydew when nectar is scarce (Konrad et al., 2009; Vosteen et al., 2016).

Because honeydew is produced by insects that feed on phloem, it can contain plant secondary metabolites that are excreted by these phloem feeders (Züst and Agrawal, 2015). Since neonicotinoids are transported through the phloem, honeydew may be an important source of these insecticides in the environment. This, however, has remained unexplored. Here, we investigated whether honeydew excreted by phloemfeeding insects contains neonicotinoid residues that can affect insects feeding on it. The presence of insecticide in honeydew would elucidate a novel route of insecticide exposure to the many organisms that feed on honeydew. To this aim, the hoverfly Sphaerophoria rueppellii, which is a pollinator in the adult stage and a predator in the juvenile stage, and the hymenopteran parasitic wasp Anagyrus pseudococci were fed ad libitum with honeydew excreted by Planococcus citri settled on one-year-old citrus trees. Infested trees were treated with the neonicotinoids thiamethoxam (trade name Actara 25WG) and imidacloprid (trade name Confidor 20LS) under two potential scenarios. To test the most common mode of application, insecticides were applied via the soil at the recommended concentrations. In a second scenario, insecticides were applied as a foliar spray at $50 \%$ of the recommended concentrations to test the effects when low doses of neonicotinoids reach honeydew producers. This second scenario 
represents exposure through i) insecticide drift to untreated plots, ii) partial exposure to insecticide when a spray does not reach all parts of the plant due to incorrect insecticide application or unfavorable climatic conditions, or iii) when neonicotinoids remain in the plant for long periods at lower concentrations (Byrne et al., 2014; Rondeau et al., 2015). Neonicotinoids can remain in plants for several months (Bonmatin et al., 2015; Byrne et al., 2012) and even for more than one to three years after the application in perennial crops such as citrus (Byrne et al., 2014; Cowles et al., 2006). During this long period, hemipterans can feed on plants and excrete honeydew contaminated with neonicotinoids at different concentrations that may cause lethal and sublethal effects on beneficial insects. Moreover, a recent study has demonstrated that neonicotinoids are present in lower than recommended rates in $93 \%$ of organic soils and crops, that had not been treated with neonicotinoids for the last 10 years (Humann-Guilleminot et al., 2019). The presence and concentration of imidacloprid and thiamethoxam in the honeydew samples were further analysed for both soil and foliar-treated trees using LC-MS/MS.

\section{RESULTS AND DISCUSSION}

\section{I. Toxicity of honeydew for hoverflies}

In soil-treated trees, $73.3 \pm 8.3 \%$ of the hoverflies died within three days of feeding on honeydew excreted by mealybugs feeding on thiamethoxam-treated trees, $33.3 \pm 8.8 \%$ of the hoverflies died in the imidacloprid treatment and $13.8 \pm 6.5 \%$ in the control treatment (GLM based on binomial distribution, $\chi_{86}^{2}=23.86, P<0.0001$ ) (Figure 1A, left panel) (SI Appendix). The corrected mortality was $69.1 \%$ for the hoverflies fed on honeydew excreted by mealybugs feeding on thiamethoxam treated trees. The longevity of the surviving hoverflies was assessed daily when they had continuous access to honeydew of the different treatments. After these three days, longevity of hoverflies fed on control honeydew (9.9 \pm 0.9 days) or honeydew from mealybugs fed on imidacloprid-treated trees $\left(8.3 \pm 0.7\right.$ days) was similar (Cox's Proportional Hazards: $\chi_{39}^{2}=2.97, P=0.085$ ) (Figure 2A, left panel).

In foliar-treated trees, all hoverflies died within three days of feeding on honeydew excreted by mealybugs feeding on thiamethoxam-treated trees, $53.5 \pm 10 \%$ of the hoverflies died in the imidacloprid treatment and only $10 \pm 6 \%$ in the control treatment (GLM based on quasi-binomial distribution, $F_{2,87}=46.22, P<0.0001$ ) (Figure 1A, right panel). The corrected mortality was $100 \%$ and $48.4 \%$ for the hoverflies fed on honeydew excreted by mealybugs feeding on thiamethoxam and imidacloprid-treated trees, respectively. After these three days, hoverflies that fed on honeydew excreted 
by mealybugs feeding on trees treated with imidacloprid ( $8.4 \pm 0.7$ days) lived significantly shorter than those fed on honeydew produced on control trees (11.3 \pm 0.6 days) (Cox's Proportional Hazards: $\chi_{1}^{2}=7.68, P=0.0056$ ) (Figure 2A, right panel). The different translocation routes of the two insecticides in the plant might explain the differential toxicity of honeydew excreted by mealybugs feeding on trees treated with thiamethoxam or imidacloprid. Thiamethoxam is a phloem-transported insecticide whereas imidacloprid is translocated mostly via xylem (Nauen et al., 2003; Weichel and Nauen, 2004). Therefore, phloem feeders such as $P$. citri are more likely to excrete thiamethoxam in their honeydew.
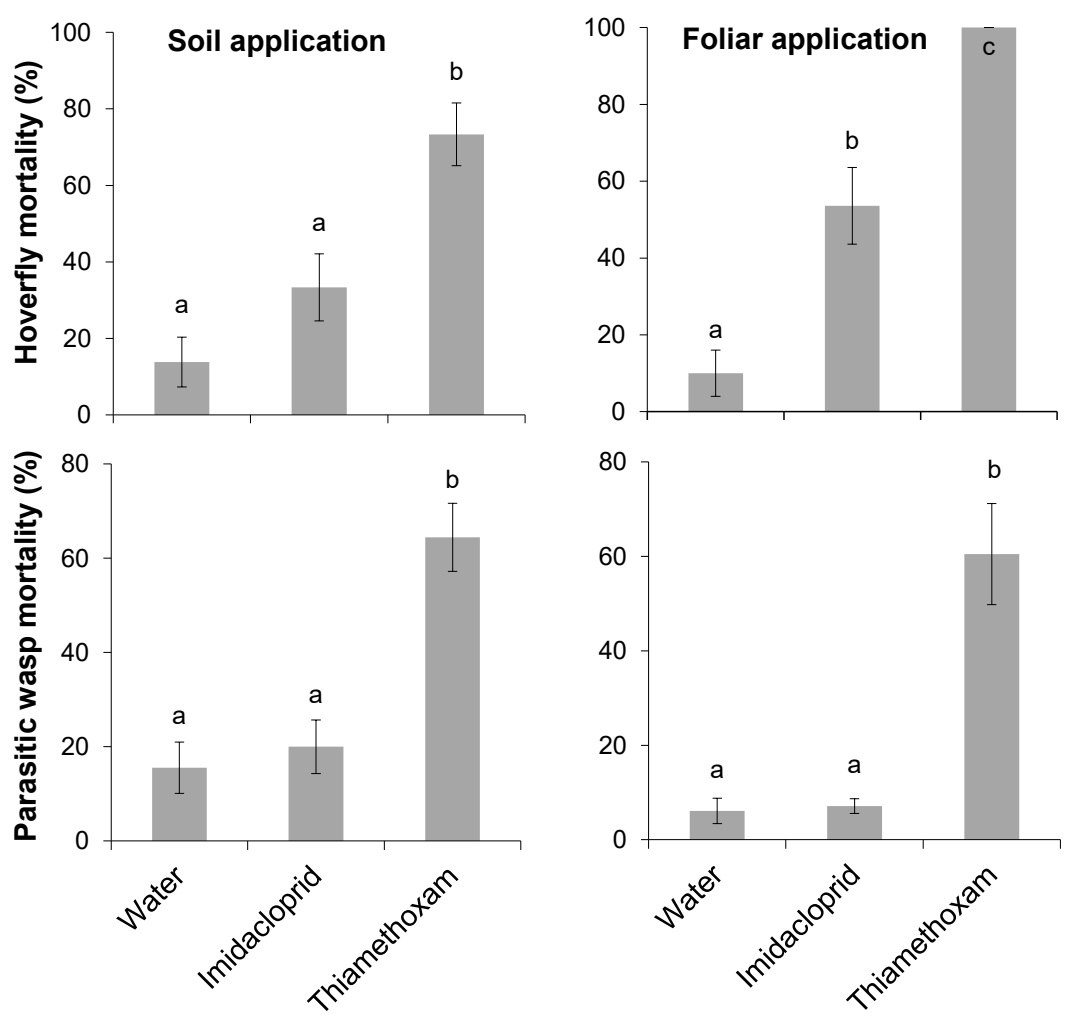

Source of honeydew

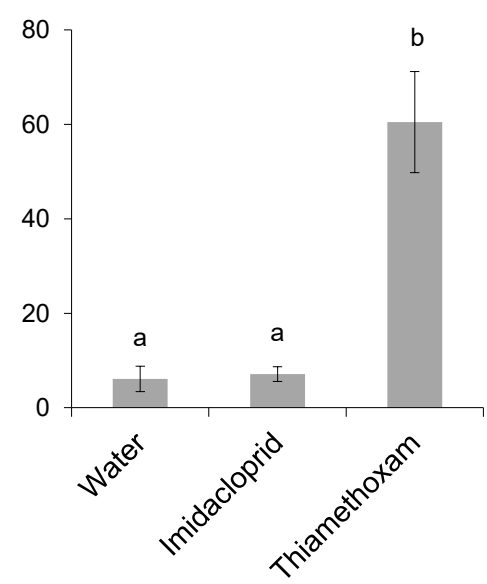

Source of honeydew

Figure 1 | Mortality of beneficial insects fed on honeydew contaminated with neonicotinoid insecticides. Mortality (mean \pm SE) of A) the pollinating hoverfly Sphaerophoria rueppellii and B) the parasitic wasp Anagyrus pseudococci fed on honeydew of Planococcus citri feeding on water-treated trees or on honeydew of $P$. citri feeding on soil- (left panels) or foliar-treated trees (right panels) with the neonicotinoid insecticides imidacloprid or thiamethoxam. Mortality was assessed after feeding on honeydew during 72 hours. Columns sharing the same letter are not significantly different from each other (Bonferroni test, $P<0.05$ ). 
Soil application
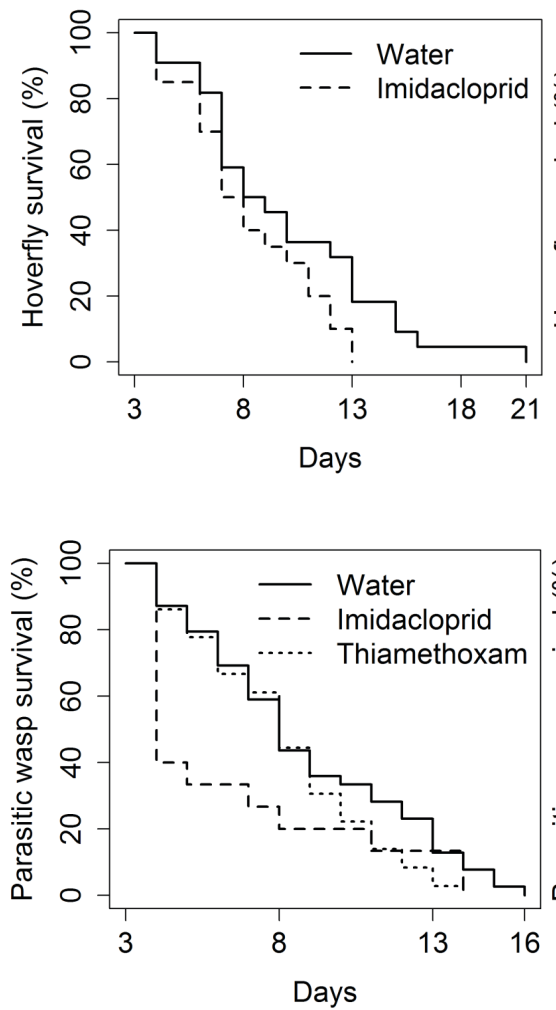

Foliar application
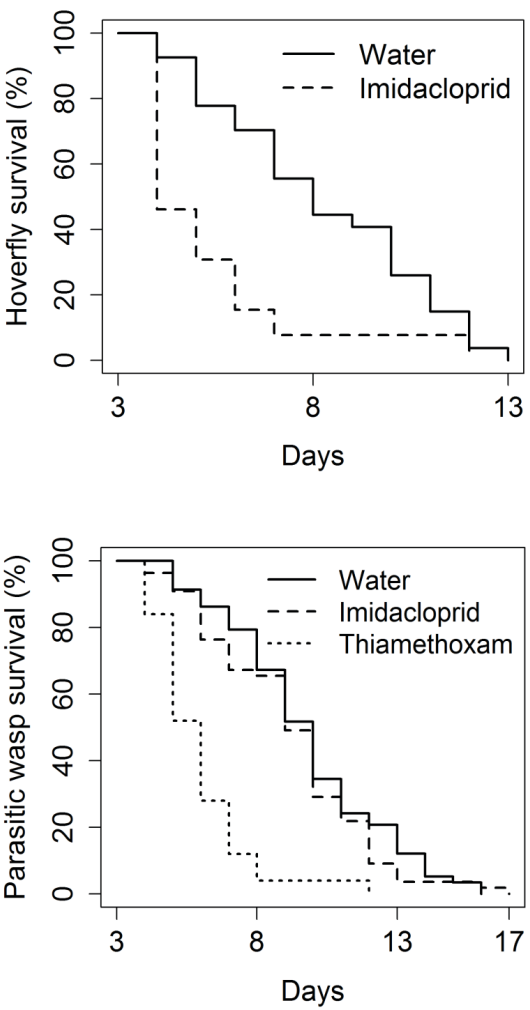

Figure 2 | Survival of beneficial insects fed on honeydew contaminated with neonicotinoid insecticides. Survival curves estimated by Kaplan-Meier of A) the pollinating hoverfly Sphaerophoria rueppellii, and B), the parasitic wasp Anagyrus pseudococci fed on honeydew of Planococcus citri feeding on watertreated trees or on honeydew of $P$. citri feeding on soil- (left panels) or foliar-treated trees (right panels) with the neonicotinoid insecticides imidacloprid or thiamethoxam.

\subsection{Toxicity of honeydew for parasitic wasps}

In soil-treated trees, $64.4 \pm 7.2 \%$ of the parasitic wasps died within three days of feeding on honeydew excreted by mealybugs that fed on trees treated with thiamethoxam, whereas $20 \pm 5.7 \%$ died in the imidacloprid treatment. Mortality in the control was 15.6 $\pm 5.5 \%$ (GLM, based on binomial distribution, $\chi^{2}{ }_{137}=31.87, P<0.0001$ ) (Figure 1B, left panel). The corrected mortality was $59 \%$ the parasitic wasps fed on honeydew excreted by mealybugs feeding on thiamethoxam treated trees. The longevity of the surviving parasitic wasps was assessed daily while they had continuous access to honeydew of the different treatments. After these three days, longevity of parasitic wasps fed on honeydew from control trees $(8.1 \pm 0.5$ days $)$ or honeydew from mealybugs fed on imidacloprid 
(8.8 \pm 0.6 days) or thiamethoxam-treated trees $(6.33 \pm 0.95$ days $)$ was similar (Cox's Proportional Hazards: $\chi_{87}^{2}=4.48, P=0.11$ ) (Figure $2 \mathrm{~B}$, left panel).

In foliar-treated trees, $60.1 \pm 10.7 \%$ of the parasitic wasps died within three days of feeding on honeydew excreted by mealybugs that fed on trees treated with thiamethoxam, whereas only $7.1 \pm 1.5 \%$ died in the imidacloprid treatment. Mortality in the control was $6.1 \pm 2.7 \%$ (GLM, based on quasi-binomial distribution, $F_{2,27}=23.98, P<0.0001$ ) (Figure 1B, right panel). The corrected mortality was $57.4 \%$ the parasitic wasps fed on honeydew excreted by mealybugs feeding on thiamethoxam treated trees. After these three days, parasitic wasps that fed on honeydew excreted by mealybugs feeding on thiamethoxam-treated trees lived significantly shorter $(7.8 \pm 0.5$ days $)$ than those fed on control honeydew (12.1 \pm 0.4 days) or on honeydew of mealybugs that had fed on imidacloprid-treated trees ( $11.4 \pm 0.4$ days) (Cox's Proportional Hazards: $\chi_{2}^{2}=43.06, P$ $<0.0001$ ) (Figure 2B, right panel). Longevity of parasitic wasps fed on control honeydew or honeydew from mealybugs fed on imidacloprid-treated trees was similar (Figure 2B). Both neonicotinoids resulted in higher mortality in the hoverfly than in the parasitic wasp. This may be due to a greater feeding rate and/or a lower detoxification capacity of the hoverfly. For example, bumblebees are more susceptible than honeybees to ingested neonicotinoids because their feeding rate is greater (Cresswell et al., 2014). In our study, we also observed qualitatively that the hoverflies ingested more honeydew than the parasitic wasps.

\subsection{Detection of neonicotinoids in honeydew}

The presence and concentration of imidacloprid and thiamethoxam in the honeydew samples were further analysed for both soil and foliar-treated trees using LC-MS/MS (SI Appendix, Figure S1, Figure S2 and Figure S3). In soil-treated trees, thiamethoxam was detected in mealybug-produced honeydew from $71.4 \pm 18.4 \%$ of the trees sampled throughout the five days that the experiment lasted (Figure 3, and SI Appendix, Table S4). These samples contained $18.3 \pm 7.6$ nanogram of thiamethoxam / $\mathrm{mL}$ of honeydew (ppb). Imidacloprid was detected in mealybug-produced honeydew from $42.9 \pm$ $20.2 \%$ of the trees sampled throughout the five days of the experiment. These samples contained $15.6 \pm 1.4$ nanogram of imidacloprid / $\mathrm{mL}$ of honeydew (ppb). Neither thiamethoxam nor imidacloprid were detected in honeydew produced by mealybugs feeding on water-treated trees (Fisher's exact test, $P=0.031$ ). In foliar-treated trees, thiamethoxam was detected in mealybug-produced honeydew from $66.7 \pm 21.1 \%$ of the trees sampled throughout the five days that the experiment lasted (Figure 3, and SI Appendix, Table S5). Imidacloprid was detected in mealybug-produced honeydew 
from $71.4 \pm 18.4 \%$ of the trees sampled throughout the five days of the experiment (Figure 3, and SI Appendix, Table S5). These samples contained 68.1 \pm 11.6 nanogram of imidacloprid / $\mathrm{mL}$ of honeydew (ppb). As in the previous experiment, neither thiamethoxam nor imidacloprid were detected in honeydew samples collected from control trees (Fisher's exact test, $P=0.023$ ).
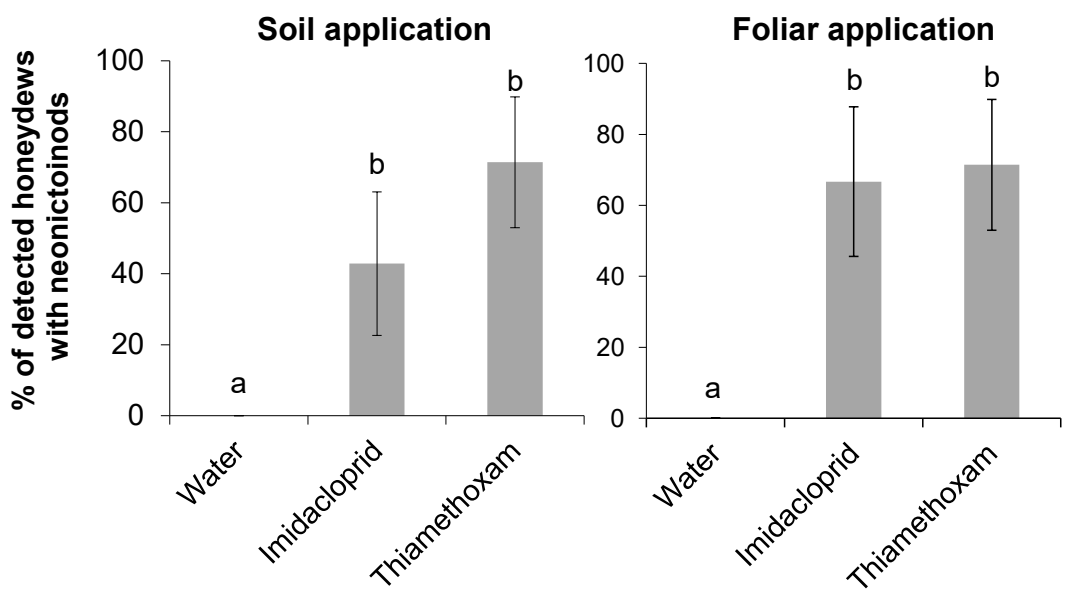

Figure 3 | Honeydew contaminated by neonicotinoid insecticides. Percentage (mean \pm SE) of soil-treated trees (left panel) or foliar-treated trees (right panel) with Planococcus citri honeydew contaminated by neonicotinoids. Neonicotinoids were detected using LC-MS/MS. Columns with different letters are significantly different from each other (Fisher's Exact Test, $P<0.05$; number of trees per treatment $=6-7)$.

Our results demonstrate that honeydew is a route of exposure to neonicotinoids for beneficial insects. Honeydew contaminated with neonicotinoids may be present in numerous ecosystems. These insecticides are used worldwide in many crops that, concurrently, are infested by honeydew producers. Moreover, these insecticides even occur in $93 \%$ of organic soils and crops, that had not been treated with neonicotinoids for the last 10 years (Humann-Guilleminot, 2019). Our study focused on citrus trees. As mentioned above, citrus is not the only crop in which neonicotinoids are routinely applied. For instance, in 2011, 79-100\% of corn and 34-44\% of soybean seeds were treated with neonicotinoids in the United States (35.1 and 32.5 million ha, respectively). These crops are infested by phloem-feeding insects that continuously excrete honeydew when they are resistant/tolerant to neonicotinoids or when neonicotinoid concentration in the plant decreases and they can feed and develop at these lower concentrations (Douglas and Tooker, 2015; Guedes et al., 2016). 
The high accessibility of honeydew excreted by numerous phloem-feeding insect species throughout the year suggests that contaminated honeydew represents a highly toxic carbohydrate source for beneficial arthropods (Lundgren, 2009; Tena et al., 2016; Wäckers et al., 2008). For example, predators (Hogervorst et al., 2008), ants (Tena et al., 2016), pollinators such as honeybees, solitary bees, bumblebees (Konrad et al., 2009; Lundgren, 2009; Tena et al., 2016) and even vertebrates like birds (Clout and Gaze, 1984) have been observed feeding on honeydew. Unavoidably, insecticides applied to control insect pests may have repercussions on organisms at different trophic levels. Insecticides taken up by lower trophic levels, i.e. herbivores, can cascade up to higher trophic levels of a food web. In addition to the direct pathway of contamination through nectar, honeydew readily drops from colonies and hence there is further potential for non-target soil-dwelling organisms to be affected via this route.

\section{CONCLUSION}

Due to the negative effects of neonicotinoids on non-target organisms, especially honeybees, the European Commission has recently banned the use of imidacloprid, thiamethoxam and clothianidin in open agroecosystems in the member states after a risk assessment report of the European Food Safety Authority (EFSA) (European Food Safety Authority, 2018). As with the previous assessments, exposure of beneficial insects to the substances was assessed via three routes: residues in bee pollen and nectar; dust drift during the sowing/application of the treated seeds; and water consumption. These decisions, however, did not consider that honeydew, which is more abundant than nectar, could be an important additional route of insecticide exposure for beneficial insects, including pollinators. This novel route of exposure is likely to affect a much wider range of beneficial insects than contaminated nectar and, thus, should be included in future environmental risk assessments.

\section{MATERIALS AND METHODS}

\section{I. Insects and experimental conditions}

The phloem-feeding herbivorous insect Planococcus citri was obtained from the State Insectary of Valencia (Almassora, Spain), where it was reared on potato sprouts and transported to the Instituto Valenciano de Investigaciones Agrarias (IVIA) (Moncada, Spain) as crawlers (first nymphal instar). The parasitic wasp Anagyrus pseudococci and the predator-pollinator Sphaerophoria rueppellii were obtained from the commercial companies Koppert Biological Systems S.L (Águilas, Spain) and Biobest Biological Systems (Westerlo, Belgium), respectively. Pupae of both species were introduced into wooden and glass rearing boxes $(51 \times 51 \times 41 \mathrm{~cm})$ with holes in the wall that were 
covered with mesh. Rearing boxes were kept in the laboratory at room temperature until adults emerged. Unfed newly emerged parasitic wasps and hoverflies were collected daily between 9:00 and 11:00 AM and used in the experiments. All experiments were carried out in different climatic chambers for each insect at $25 \pm 2{ }^{\circ} \mathrm{C}, 75 \pm 10 \% \mathrm{RH}$ and a photoperiod of 14:10h (L:D).

We selected hoverflies and parasitic wasps of honeydew-producing insects because it is known that they feed on honeydew in the field and also use honeydew as cues to locate their hosts (Calabuig et al., 2015; Franco et al., 2008; Hogervorst et al., 2007; Lee et al., 2006; Steppuhn and Wäckers, 2004; Tena et al., 2013b; Vosteen et al., 2016). Therefore, they are extensively in contact with honeydew in the field. Moreover, we selected a hoverfly because hoverflies represent one of the most important groups of pollinators (Rader et al., 2015); some genera of hoverflies are also predators during their larval stage (Jervis, 2005); and, finally, they are highly sensitive to insecticides and their populations are in decline (Hallmann et al., 2017; Sanchez-Bayo, 2014). A parasitic wasp was selected because these wasps represent one of the main groups of beneficial insects in agriculture (Heimpel and Mills, 2017; Jervis, 2005; Wajnberg et al., 2008). One of the most important examples of biological control in the world is based on Anagyrus parasitoids (Herren and Neuenschwander, 1991; Wyckhuys et al., 2018; Zeddies et al., 2001).

\subsection{Plant infestation and insecticide application}

Twenty-seven and forty-five potted clementine trees cv. Clementina de Nules grafted on 'Macrophyla' (Citrus sinensis $\times$ Poncirus trifoliata) were reared and infested for the foliar and soil insecticide applications, respectively. Trees were two-year-old and $\sim 1 \mathrm{~m}$ high and they were maintained in a greenhouse at IVIA. The environmental conditions were 22 $\pm 5^{\circ} \mathrm{C}, 70 \pm 20 \% \mathrm{RH}$ and natural photoperiod (January-April). Clementine trees were watered three times per week and were fertilized once per week with Sofertirrig ${ }^{\circledR}$ fertilizer (18-18-18 N-P-K). They were infested with Planococcus citri crawlers on February $28^{\text {th }}$, 2018 , for the foliar insecticide application and January $22^{\text {nd }}, 2017$, for the soil insecticide application. To infest them, $1.5 \mathrm{ml}$ centrifuge tubes half-filled with $P$. citri crawlers were placed on the crown of each plant.

The neonicotinoids used in this research were thiamethoxam (Thiamethoxam (25\%), Actara 25 WG, Syngenta) and imidacloprid (Imidacloprid (20\%), Confidor 20 LS, Bayer). Two potential scenarios were tested. First scenario: Insecticides were applied via the soil at the recommended concentrations to test the most common mode of application (Boina and Bloomquist, 2015; Cocuzza et al., 2017; Grafton-Cardwell et al., 2013; Qureshi et al., 
2014). For this, we applied each insecticide solution or distilled water (control treatment) to fifteen clementine plants per treatment directly on the soil on March 23. Neonicotinoids were applied onto the soil at the dose recommended by the producer (Syngenta, 2019; Bayer Crop Science, 2019). A concentration of $0.3 \mathrm{~g}$ of a.i. of thiamethoxam/ $1 \mathrm{~L}$ of distilled water or $0.75 \mathrm{ml}$ of imidacloprid / $1 \mathrm{~L}$ of distilled water was applied on fifteen different plants per treatment. Untreated controls were watered using only distilled water. We used different $0.5 \mathrm{~L}$ glass jars for each treatment to water plants.

Second scenario: Insecticides were applied as a foliar spray at $50 \%$ of the recommended concentrations to test the effects when low doses of neonicotinoids reach honeydew producers. For this, we applied each insecticide or distilled water (control treatment) in separate chambers to nine clementine plants per treatment on April 19. Plants were temporarily removed from the greenhouse in order to prevent spray drift and crosscontamination of treatments. Neonicotinoids were applied onto the foliage at half the dose recommended by the producer (Syngenta, 2019; Bayer Crop Science, 2019). A concentration of $0.1 \mathrm{~g}$ of thiamethoxam / $1 \mathrm{~L}$ of distilled water and a concentration of $0.15 \mathrm{ml}$ of imidacloprid / $1 \mathrm{~L}$ of distilled water were applied on nine different plants per treatment. Untreated controls were sprayed using only distilled water. We used $2 \mathrm{~L}$ manual sprayers and a separate sprayer was used for each insecticide and the control. Insecticides were sprayed until run-off $(200 \mathrm{ml})$. One hour after spraying, we returned the trees to their previous positions in the greenhouse.

\subsection{Honeydew collection}

For soil application, we collected honeydew daily from March 24 (+1 days after treatment, DAT) to March 29 ( +5 DAT) by placing Parafilm ${ }^{\circledR}$ squares of $10 \mathrm{~cm} \times 10 \mathrm{~cm}$ below the plant for 24 hours. The collected honeydew for each treatment was labelled and stored at $-20{ }^{\circ} \mathrm{C}$ in Petri dishes until they were used (Hogervorst et al., 2007). Honeydew was labelled with information on treatment, tree number, and day of collection. The same procedure was carried out for the foliar application experiment from April 20 to 25.

\subsection{Amount of honeydew produced by the mealybugs and provided to the hoverflies and parasitic wasps.}

For the soil application experiment, the amount of honeydew produced by $P$. citri and the honeydew provided to the beneficial insects, the hoverfly $S$. rueppellii and the parasitic wasp A. pseudococci, was estimated. The amount of honeydew produced by $P$. citri per treatment and per day (1, 3, 5 and 10 DAT) in each tree was assessed by counting, under a stereo microscope, the total number of small (less than $150 \mu \mathrm{m} \varnothing$ ), medium (between 150 and 
$300 \mu \mathrm{m} \varnothing$ ) and large (more than $300 \mu \mathrm{m} \varnothing$ ) honeydew droplets on three squares of $1 \mathrm{~cm}^{2}$ each, for three randomly collected $25 \mathrm{~cm}^{2}$ Parafilm ${ }^{\oplus}$ pieces from the same tree and day. The volume of each categorized droplet was estimated as $\left(4 / 3 \times \pi \times r^{3}\right) \times 1 / 2$, where $r$ is the radius of the droplet. Subsequently, we estimated the total volume of honeydew for each $1 \mathrm{~cm}^{2}$ section by summing up the volume of all counted droplets (SI Appendix, Table S1).

To ensure that all insects received honeydew ad libitum in the toxicity assay, the amount of honeydew provided was estimated. The mean volume of honeydew per $\mathrm{cm}^{2}$ of Parafilm in each treatment was multiplied by the area of Parafilm ${ }^{\circledR}$ provided per day (SI Appendix, Table S2). The corresponding honeydew-containing Parafilm ${ }^{\circledR}$ sections were placed in the Petri dish or glass vials together with wet cotton wool. For all experiments, honeydew was renewed daily to avoid crystallization (Hogervorst et al., 2007; Tena et al., 2013b 3).

\subsection{Toxicity of honeydew excreted by mealybugs feeding on trees treated with neonicotinoids as assessed for hoverflies and parasitic wasps}

We fed the hoverfly S. rueppellii and the parasitic wasp A. pseudococci with honeydew excreted by $P$. citri feeding on trees that had been treated with thiamethoxam, imidacloprid or distilled water (control). For the hoverfly S. rueppellii, we confined 30 newly emerged and unfed adults individually in 5.3-cm-diameter Petri dishes with 3-cm-diameter holes covered with muslin mesh to allow ventilation. For the parasitic wasp A. pseudococci, on the soil application, between 45 and 50 parasitic wasps per treatment were used and placed individually in glass vials of $3 \mathrm{~cm}$ high and $0.8 \mathrm{~cm}$ diameter covered with wet cotton wool. Instead, for the foliar application, groups of ten newly emerged and unfed females per Petri dish were used. Ten replicates (each containing ten new parasitic wasps) per treatment were carried out (100 individuals per treatment).

For the soil and foliar application experiments, Parafilm ${ }^{\circledR}$ pieces with honeydew of each treatment were defrosted, observed under the stereo microscrope to check for the presence of honeydew, and cut into pieces of different sizes to provide honeydew ad libitum (ca. $4 \mathrm{~cm}^{2}$ for the Petri dishes and $0.5 \mathrm{~cm}^{2}$ for the glass vials). Petri dishes or glass vials containing the different beneficial insects were kept in the climatic chambers during 72 hours and afterwards mortality was assessed. Feeding beneficial insects with contaminated honeydew in a no-choice situation represents the most common scenario under field conditions because agriculture is based on large-scale uniformly-treated monocultures where floral nectar is scarce, and is limited to only the brief flowering period in flowering crops (Gurr et al., 2017). 


\subsection{Effects of honeydew excreted by mealybugs feeding on trees treated with neonicotinoids on hoverfly and parasitic wasp longevity}

After 72 hours, surviving hoverflies and parasitic wasps of each replicate were placed individually into new containers to study potential sublethal effects on longevity. The surviving hoverflies were kept in the same Petri dishes used previously for the toxicity study. For the soil application experiment, we analysed a total of 22 hoverflies fed on honeydew from mealybugs feeding on untreated trees, and 20 individuals fed on honeydew from mealybugs feeding on trees treated with imidacloprid. For the foliar application, we analysed a total of 27 hoverflies fed on honeydew from mealybugs feeding on untreated trees, and 13 fed on honeydew from mealybugs feeding on trees treated with imidacloprid. This experiment was not carried out for thiamethoxam because most individuals had died during the previous experiment.

For the parasitic wasp A. pseudococci in the soil application experiment, parasitic wasps were kept in the same glass vials used for the toxicity assay. We analysed a total of 36 parasitic wasps fed on honeydew from mealybugs feeding on trees treated with distilled water, 39 on honeydew from mealybugs feeding on trees treated with imidacloprid and 15 with thiamethoxam. For the foliar application experiment, between one and seven surviving females per replicate were placed individually into glass vials (subreplicates). Each surviving female was used as replicate because there were no significant differences between replicates (females coming from the same Petri dish) in any treatment: Survivorship of parasitic wasp females fed on honeydew excreted by mealybugs feeding on trees treated with water $\left(\chi_{9}^{2}=10.1, P=0.34\right)$; mealybugs feeding on trees treated with imidacloprid $\left(\chi_{9}^{2}=13.53, P=0.16\right)$ or thiamethoxam $\left(\chi_{7}^{2}=9.96, P=0.19\right)$ (number of individuals per replicate in SI Appendix, Table S3). Therefore, we analysed a total of 58 parasitic wasps fed on honeydew from mealybugs feeding on trees treated with distilled water only, 55 on honeydew from mealybugs feeding on trees treated with imidacloprid and 25 with thiamethoxam

Diets were provided ad libitum daily for each treatment and experiment on both beneficial insects. We checked survival daily until all adults had died. Glass vials and Petri dishes were kept in the climate chambers until all hoverflies and parasitic wasps had died.

\subsection{Neonicotinoid detection in honeydew samples}

After feeding the beneficial insects, the remaining honeydew for both insecticide applications experiment was used to assess the presence of insecticide. For the soil-treated trees, we analysed seven samples from each treatment as follows: control honeydew 
(excreted by mealybugs feeding on water-treated trees), samples of honeydew excreted by mealybugs feeding on trees treated with imidacloprid, honeydew excreted by mealybugs feeding on trees treated with thiamethoxam (SI Appendix, Table S4). For the foliartreated trees, we analysed eight samples of control honeydew (excreted by mealybugs feeding on water-treated trees) coming from five trees and three days; 17 samples of honeydew excreted by mealybugs feeding on trees treated with imidacloprid from seven trees and five days and 14 of honeydew excreted by mealybugs feeding on trees treated with thiamethoxam from six trees and five different days (SI Appendix,Table S5). Each sample comprised the remaining honeydew for tree and day. The amount of honeydew per sample was assessed as explained in the section "Amount of honeydew produced by mealybugs and provided to the hoverflies and parasitic wasps". Then, we extrapolated this value to estimate the total volume of honeydew on the Parafilm ${ }^{\oplus}\left(25 \mathrm{~cm}^{2}\right)$.

\subsection{Chemicals}

High purity (98-99.9\%) standards of desired insecticides, namely, imidacloprid, thiamethoxam and its metabolite clothianidin were purchased from Sigma-Aldrich (Steinheim, Germany). Individual standard solutions were prepared in methanol at a concentration of $1 \mathrm{~g} \cdot \mathrm{L}^{-}$ 1. The working standard solution was prepared by mixing the appropriate amounts of individual standard solutions and diluting with methanol to a final concentration of 0.5 $\mathrm{mg} \cdot \mathrm{L}^{-1}$. All solutions were stored in $10 \mathrm{~mL}$ glass vials at $4{ }^{\circ} \mathrm{C}$ in the dark.

Ammonium formate and methanol (gradient grade for liquid chromatography) were obtained from Sigma-Aldrich (Steinheim, Germany) and Panreac (Darmstadt, Germany), respectively. High purity water was prepared using a Milli-Q water purification system (Millipore, Milford, MA, USA). Ten millimolar ammonium formate solutions prepared in both Milli-Q water and methanol were used as mobile phase in LC-MS/MS.

\subsubsection{Insecticide extraction from honeydew}

All droplets of honeydew from the same tree and day were dissolved in 'Sample Diluent Buffer' (Imidacloprid ELISA, Microtiter Plate-kit, Abaraxis. Inc.) in case of foliar-treated trees or in $50 \%$ methanol in case of soil-treated trees. One hundred microliters of diluent solution were ejected on top of the Parafilm ${ }^{\circledR}$ piece containing the honeydew droplets. The diluent solution and the honeydew droplets were stirred gently with the same pipette to dissolve the honeydew and then draw into Eppendorf tubes. In the case of samples dissolved with 'Sample Diluent Buffer', these $100 \mu \mathrm{L}$ were mixed with $100 \mu \mathrm{L}$ of methanol and injected in the LC-MS/MS. The samples dissolved with 50\% methanol were used without further dilution to inject in the LC-MS/MS. 


\subsubsection{Chemical analysis using Liquid Chromatography-Mass Spectrometry (LC-MS/MS)}

The chromatographic instrument was an HP1200 series LC equipped with an automatic injector, a degasser, a quaternary pump, and a column oven-combined with an Agilent 6410 triple quadrupole (QQQ) mass spectrometer with an electrospray ionization (ESI) interface (Agilent Technologies, Waldbronn, Germany). Data were processed using a MassHunter Workstation Software for qualitative and quantitative analysis (GL Sciences, Tokio, Japan). The chromatographic column was a Luna C18 $(15.0 \mathrm{~cm} \times 0.21 \mathrm{~cm})$ with a $3 \mu \mathrm{m}$ particle size (Phenomenex, Torrance, USA). The column temperature was kept at $30{ }^{\circ} \mathrm{C}$ and the volume injected was $5 \mu \mathrm{L}$. An isocratic binary mobile phase consisted of $10 \mathrm{mM}$ ammonium formate: in Milli-Q water and in methanol (50:50, v/v) at flow rate of $0.3 \mathrm{~mL} \cdot \mathrm{min}^{-1}$ was used.

The ESI ionization source parameters were drying gas (nitrogen) flow of $11 \mathrm{~L} \mathrm{~min}^{-1}$ at temperature of $300{ }^{\circ} \mathrm{C}$, nebulizer pressure of 15 psi (1034.2 mbar) and capillarity voltage of $4000 \mathrm{~V}$. The triple quadrupole worked in Multiple Reaction Monitoring (MRM) with both mass spectrometers at unit resolution and a dwell time of $10 \mathrm{~ms}$ and a cell accelerator voltage of $7 \mathrm{eV}$. The particular conditions to determine each insecticide are specified in SI Appendix, Table S6.

\subsubsection{Method validation and quality control}

The linearity of the MS/MS method was established with six calibration points, using external standards over a concentration range of $1-250 \mathrm{ng} \cdot \mathrm{mL}^{-1}$ (equivalent to $2-500 \mathrm{ng} \cdot \mathrm{g}^{-1}$ in the extract). The peak area of target analytes was calculated using Mass Hunter software (Agilent). Each point was obtained as the mean of three independent injections. The data were fit to a linear least-squares regression curve with a $1 / x$ weighting that was not forced through the origin. The calibration curves were $y=359 x-42$ for thiamethoxam, $y=129 x$ +83 for imidacloprid and $y=132 x+27$ for clothianidin. All of them provided an $\mathrm{r}^{2}>0.99$.

The sensitivity of the method was estimated by establishing the limits of detection (LODs) and quantification (LOQs) using standard solutions prepared in spiked honey samples that were free of insecticides. The LODs were established as the lowest insecticide concentration whose qualified transition $\left(\mathrm{SRM}_{2}\right)$ presented a signal-to-noise ratio $(\mathrm{S} / \mathrm{N})$ $\geq 3$. They were $0.05,0.03$ and $0.04 \mathrm{ng} / \mathrm{mL}$ of extract for thiamethoxam, imidacloprid and clothianidin, respectively. The LOQs were determined also in pure solvent and in spiked honey as the minimum detectable amount of analyte with $S / N \geq 10$ for the quantifier $\left(\mathrm{SRM}_{1}\right)$ transition. All the LOQs were verified spiking the samples and analysing them. They were $0.15,0.1$ and $0.12 \mathrm{ng} / \mathrm{mL}$ of extract for thiamethoxam, imidacloprid and 
clothianidin, respectively. This level of sensitivity allowed the detection and quantification of very low amount of insecticide in the extracts that might be coming from residual contaminations from previous treatments of the trees used in the experiments. In case of soil-treated trees, we have detected imidacloprid in some of the water-treated trees with levels ranging from $<\mathrm{LOQ}$ to $0.5 \mathrm{ng} \mathrm{mL}^{-1}$. Hence, and for the sake of accuracy, we have subtracted $0.5 \mathrm{ng} \mathrm{mL}^{-1}$ to all imidacloprid values in this experiment (SI Appendix, Table S4) (Calatayud-Vernich et al., 2016; Masiá et al., 2013).

\subsection{Statistical analysis}

To analyse the mortality of the parasitic wasp and the hoverfly after feeding on honeydew for three days, we used a generalized linear model with binomial distribution (soil application) or quasi-binomial distribution (foliar application) of females after 72 hours of feeding on honeydew. The mortality of the parasitic wasps in the foliar insecticide application was calculated as the number of dead parasitic wasps divided by total number of parasitic wasps per Petri dish. In both analyses, honeydew type was the explanatory variable and mortality the dependent variable. A Bonferroni post-hoc test using "multcomp" package enabled pairwise comparisons between honeydew treatments. When significant differences between the control and the treated honeydews were found $(P<0.05)$, mortality was corrected using the Abbott formula. The effect of the honeydew treatments on the parasitic wasp or hoverfly survivorship was represented by KaplanMeier survivorship curves and analysed by a log-rank test using the survival functions of the "Survival" package. The percentage of trees in which neonicotinoids were detected in the collected honeydew was analysed using a Fisher's exact test. All tests performed were analysed using the computer programme R (version 3.3.2 for Macintosh).

\section{ACKNOWLEDGEMENTS}

We thank Biobest Biological Systems and Koppert Biological Systems for providing the beneficial arthropods used during the bioassays and the State Insectary of Valencia for providing P. citri. We acknowledge Dr. F.L. Wäckers for the recommendation to use the hoverfly in the assays. We also acknowledge P. Bru, J. Catalán, E. Rubio and P. Cuenca for their valuable help during the experiments, $\mathrm{M}$. Cendoya for helping during the statistical analysis and Dr. Wim van der Putten for comments on a previous version of the manuscript. This research was partially funded by an INIA project (Project No. RTA2017-00095) and the Conselleria d'Agricultura, Pesca i Alimentació de la Generalitat Valenciana. J.G.C was supported by the Spanish Ministry of Economy and Competitiveness, Ramón y Cajal Program (RYC-2013-13834) and M.C-A was recipient of grant from INIA (CPD20160085). Authors declare no competing interests. 


\section{SUPPORTING INFORMATION}
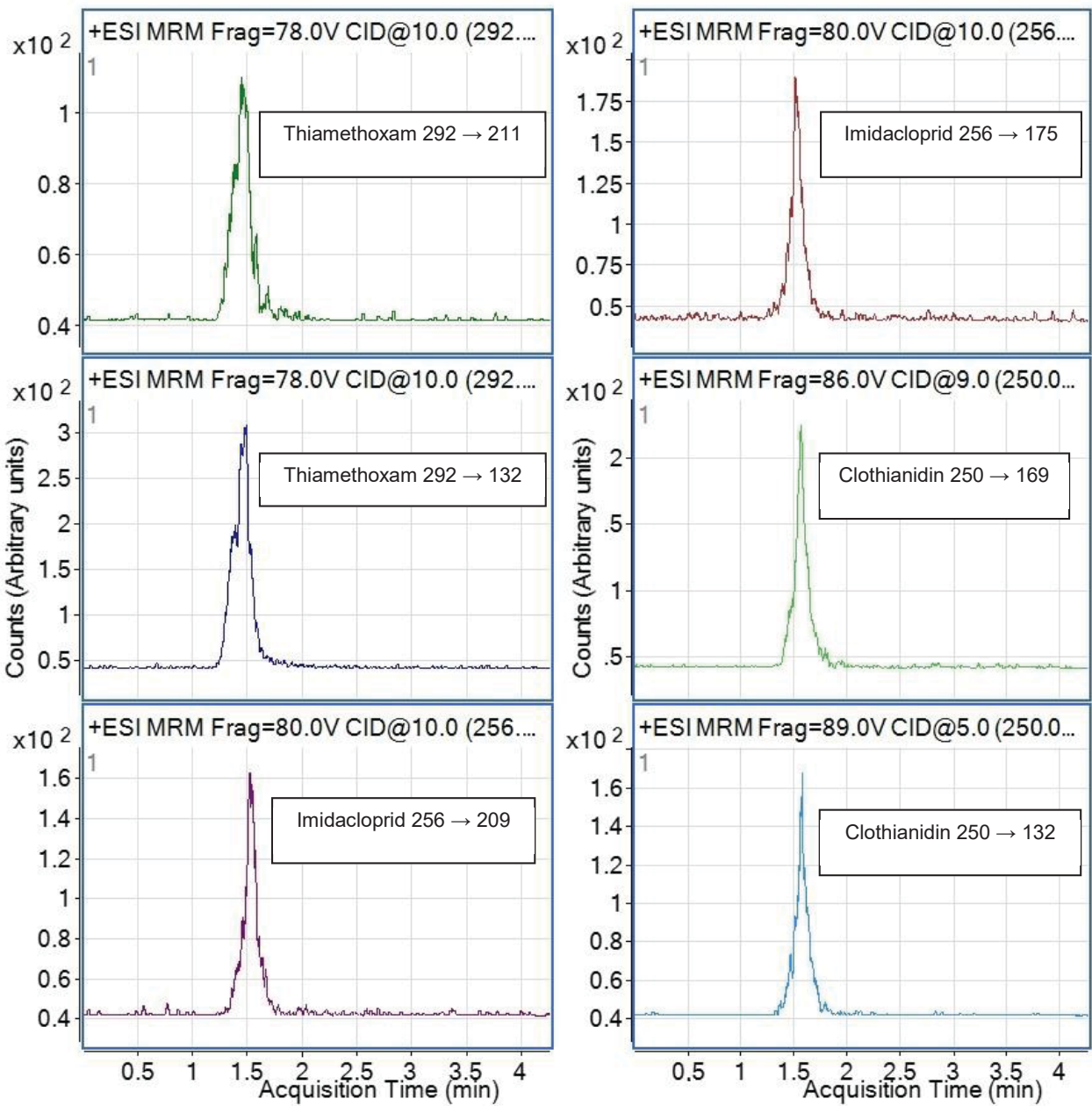

Figure S1 | Extracted ion LC-MS/MS chromatogram of an analytical standard of thiamethoxam, imidacloprid and clothianidin (metabolite of thiamethoxam) at $10 \mathrm{ng} \mathrm{mL}{ }^{-1}$ ach. Each panel shows one extracted ion chromatogram that means one precursor ion $\rightarrow$ product ion transition (two chromatograms per compound). 

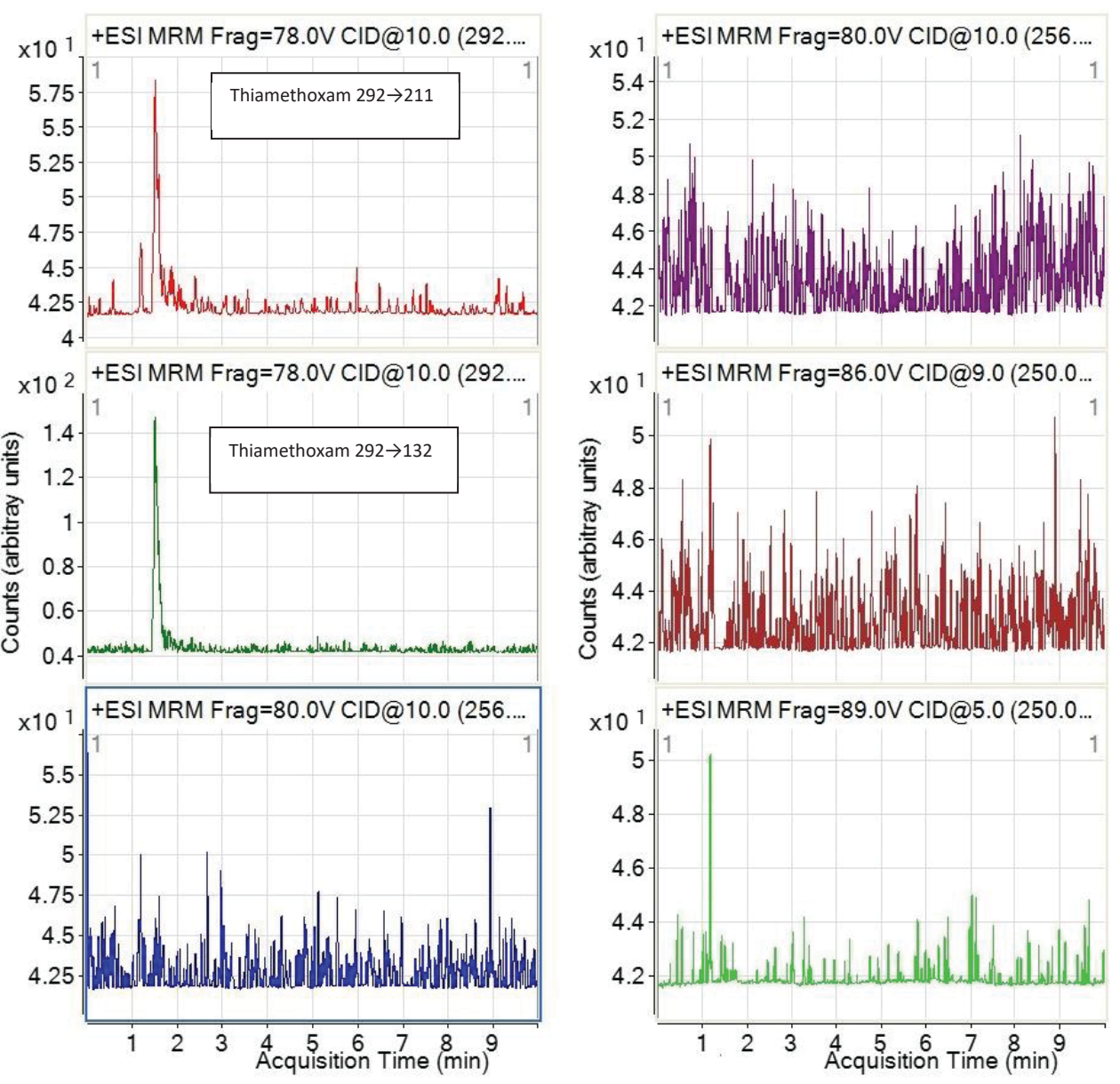

Figure S2 | Extracted LC-MS/MS chromatogram of a sample extract containing thiamethoxam at $2 \mathrm{ng}$ $\mathrm{mL}^{-1}$. Each panel shows one extracted ion chromatogram that means one precursor ion $\rightarrow$ product ion transition (two chromatograms per compound) as in Figure S1. Here only the compounds that provide a signal are labeled. 

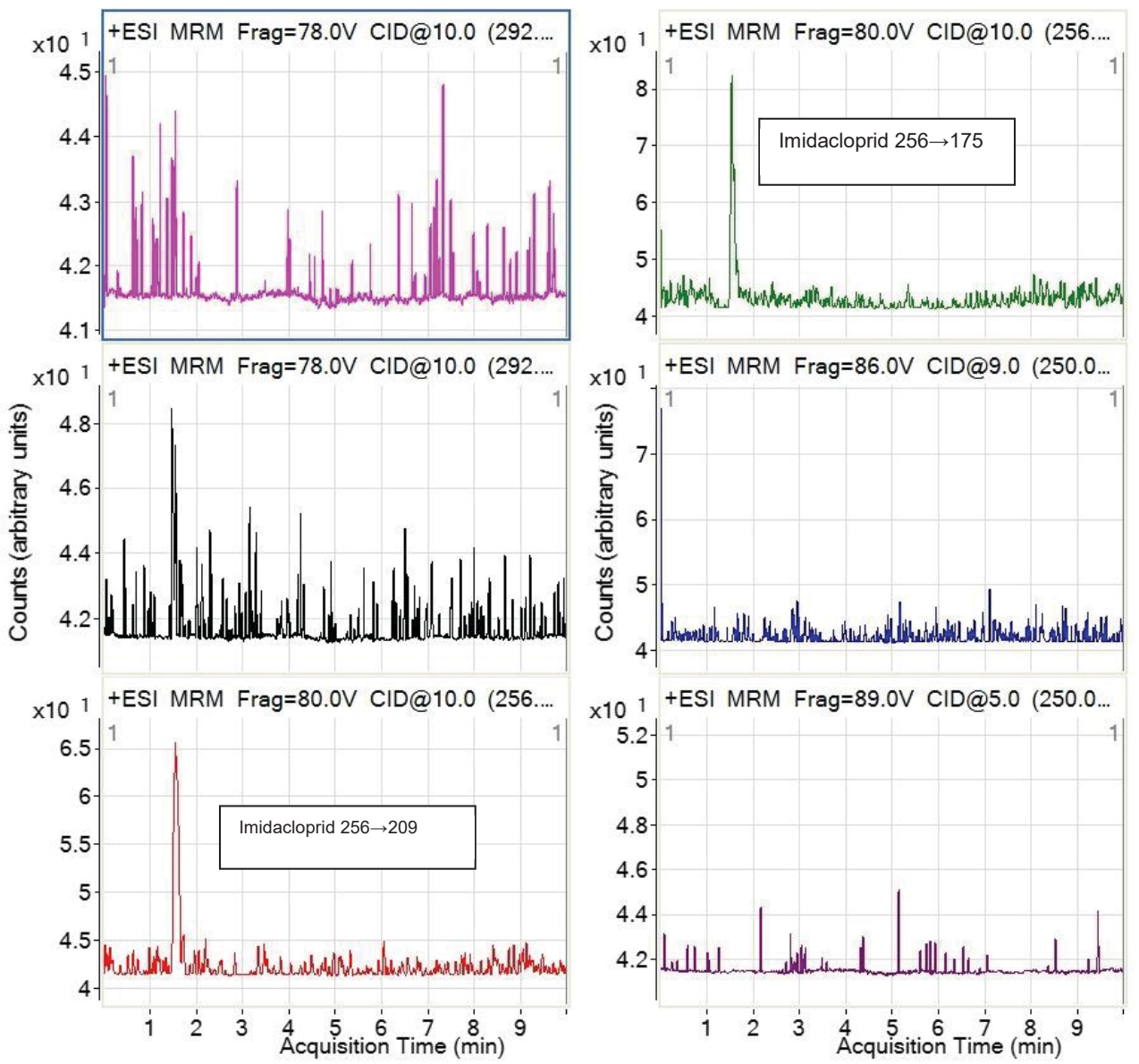

Figure S3 | Extracted ion LC-MS/MS chromatogram of a sample extract containing Imidacloprid at 3 $\mathrm{ng} \mathrm{mL} \mathrm{m}^{-1}$. Each panel shows one extracted ion chromatogram that means one precursor ion $\rightarrow$ product ion transition (two chromatograms per compound) as in Figure S1. Here only the compounds that provide a signal are labelled. 
Table S1 | Mean daily amount of honeydew ( \pm SE) produced by Planococcus citri throughout the experiment.

\begin{tabular}{lccccc}
\hline \multirow{2}{*}{ Treatments } & \multicolumn{4}{c}{ Mean honeydew $(\mu \mathrm{l})$ per $\mathrm{cm}^{2}$} & \\
& Day I & Day 3 & Day 5 & Day 10 & Mean days I-3 \\
\hline $\begin{array}{l}\text { Control honeydew } \\
\begin{array}{l}\text { Honeydew with } \\
\text { imidacloprid }\end{array}\end{array}$ & $0.16 \pm 0.04$ & $0.19 \pm 0.05$ & $0.17 \pm 0.02$ & $0.37 \pm 0.1$ & $0.18 \pm 0.01$ \\
$\begin{array}{l}\text { Honeydew with } \\
\text { thiamethoxam }\end{array}$ & $0.15 \pm 0.04$ & $0.19 \pm 0.05$ & $0.14 \pm 0.01$ & $0.03 \pm 0.01$ & $0.17 \pm 0.02$ \\
\hline
\end{tabular}

Table S2 | Mean amount of honeydew $( \pm$ SE) provided to the hoverfly Sphaerophoria rueppellii and the parasitic wasp Anagyrus pseudococci per day.

\begin{tabular}{|c|c|c|c|c|}
\hline $\begin{array}{l}\text { Beneficial } \\
\text { insect }\end{array}$ & Treatment & $\begin{array}{l}\text { Mean } \\
\text { honeydew } \\
\text { produced } \\
\left(\mu \mathrm{l} / \mathrm{cm}^{2}\right)^{*}\end{array}$ & $\begin{array}{l}\text { Number of } \\
\text { parafilm pieces } \\
\text { provided to } \\
\text { the beneficial } \\
\text { insect }^{* *}\end{array}$ & $\begin{array}{l}\text { Amount of } \\
\text { honeydew } \\
\text { provided to the } \\
\text { beneficial } \\
\text { insect }(\mu l)^{* *+*}\end{array}$ \\
\hline \multirow{3}{*}{ Hoverfly } & Control honeydew & $0.18 \pm 0.01$ & 3 & $2.10 \pm 0.18$ \\
\hline & Honeydew with imidacloprid & $0.17 \pm 0.02$ & 3 & $2.04 \pm 0.29$ \\
\hline & Honeydew with thiamethoxam & $0.065 \pm 0.01$ & 7 & $1.82 \pm 0.23$ \\
\hline \multirow{3}{*}{$\begin{array}{l}\text { Parasitic } \\
\text { wasp }\end{array}$} & Control honeydew & $0.18 \pm 0.01$ & 3 & $0.26 \pm 0.02$ \\
\hline & Honeydew with imidacloprid & $0.17 \pm 0.02$ & 3 & $0.25 \pm 0.03$ \\
\hline & Honeydew with thiamethoxam & $0.065 \pm 0.01$ & 6 & $0.20 \pm 0.02$ \\
\hline
\end{tabular}

\footnotetext{
* Data obtained from Table S1.

${ }^{* *}$ Area of the Parafilm ${ }^{\oplus}$ pieces provided to the hoverfly: $4 \mathrm{~cm}^{2}$. Area of the Parafilm pieces provided to the hoverfly: $0.5 \mathrm{~cm}^{2}$

*** Statistics (ANOVA) for hoverfly: $F_{1,34}=1.58 ; P=0.217$; for parasitic wasp: $F_{1,34}=1.55 ; P=0.22$
} 
Table S3 | Number of Anagyrus pseudococci used in the survival assay for each treatment and the initial replicate from where they were used.

\begin{tabular}{lccc}
\hline Replicate & Control honeydew & $\begin{array}{c}\text { Treatment } \\
\text { Honeydew with } \\
\text { imidacloprid }\end{array}$ & $\begin{array}{c}\text { Honeydew with } \\
\text { thiamethoxam }\end{array}$ \\
\hline $\mathbf{1}$ & 6 & 7 & 3 \\
$\mathbf{2}$ & 6 & 5 & 1 \\
$\mathbf{3}$ & 3 & 6 & 2 \\
$\mathbf{4}$ & 6 & 3 & 6 \\
$\mathbf{5}$ & 7 & 4 & 2 \\
$\mathbf{6}$ & 5 & 6 & 2 \\
$\mathbf{7}$ & 6 & 5 & 0 \\
$\mathbf{8}$ & 7 & 7 & 0 \\
$\mathbf{9}$ & 5 & 6 & 7 \\
$\mathbf{1 0}$ & 7 & 6 & 2 \\
\hline Total & $\mathbf{5 8}$ & $\mathbf{5 5}$ & $\mathbf{2 5}$ \\
\hline
\end{tabular}


Table S4 | Neonicotinoid detection for each treatment, day and tree in the soil-treated trees.

\begin{tabular}{|c|c|c|c|c|c|}
\hline Treatment & $\begin{array}{l}\text { Day after } \\
\text { treatment }\end{array}$ & $\begin{array}{c}\text { Tree } \\
\text { number }\end{array}$ & $\begin{array}{l}\text { Neonicotinoid } \\
\text { concentration } \\
\text { in the extract } \\
\left(\mathrm{ng} \mathrm{mL} \mathrm{mL}^{-1}\right)\end{array}$ & $\begin{array}{l}\text { Honeydew } \\
\text { volume } \\
\left(\mathrm{mm}^{3}\right)\end{array}$ & $\begin{array}{c}\text { Neonicotinoid } \\
\text { concentration in } \\
\text { honeydew volume } \\
\left(\mathrm{ng} \mathrm{mL} \mathrm{L}^{-1}\right)\end{array}$ \\
\hline \multirow{7}{*}{ Water } & 3 & 1 & 0 & 19.70 & 0 \\
\hline & 3 & 2 & 0 & 11.63 & 0 \\
\hline & 3 & 3 & 0 & 30.85 & 0 \\
\hline & 3 & 4 & 0 & 8.64 & 0 \\
\hline & 3 & 5 & 0 & 7.46 & 0 \\
\hline & 3 & 6 & 0 & 10.66 & 0 \\
\hline & 3 & 7 & 0 & 10.04 & 0 \\
\hline \multirow{7}{*}{ Imidacloprid } & 3 & 1 & 1.6 & 24.04 & 13.31 \\
\hline & 3 & 2 & 0 & 15.43 & 0 \\
\hline & 3 & 3 & 0.75 & 9.82 & 15.26 \\
\hline & 3 & 4 & 0 & 24.98 & 0 \\
\hline & 3 & 5 & 1.29 & 14.19 & 18.17 \\
\hline & 3 & 6 & 0 & 12.17 & 0 \\
\hline & 3 & 7 & 0 & 2.97 & 0 \\
\hline \multirow{7}{*}{ Thiamethoxam } & 3 & 1 & 0.56 & 4.18 & 26.76 \\
\hline & 3 & 2 & 0.18 & 0.83 & 43.11 \\
\hline & 3 & 3 & 0.1 & 4.08 & 4.89 \\
\hline & 3 & 4 & 0.11 & 16.16 & 1.86 \\
\hline & 3 & 5 & 0 & 0.76 & 0 \\
\hline & 3 & 6 & 0 & 1.20 & 0 \\
\hline & 3 & 7 & 0.28 & 3.82 & 14.67 \\
\hline
\end{tabular}


Table S5 | Neonicotinoid detection for each treatment, day and tree in the foliar-treated trees.

\begin{tabular}{|c|c|c|c|c|c|}
\hline Treatment & $\begin{array}{l}\text { Day after } \\
\text { treatment }\end{array}$ & $\begin{array}{c}\text { Tree } \\
\text { number }\end{array}$ & $\begin{array}{l}\text { Neonicotinoid } \\
\text { concentration } \\
\text { (ppb) }\end{array}$ & $\begin{array}{l}\text { Honeydew } \\
\text { volume } \\
\left(\mathrm{mm}^{3}\right)\end{array}$ & $\begin{array}{c}\text { Neonicotinoid } \\
\text { concentration } \\
\text { based on honeydew } \\
\text { volume (ppb) }\end{array}$ \\
\hline \multirow{8}{*}{ Water } & 3 & 7 & 0 & 7.51 & 0 \\
\hline & 4 & 1 & 0 & 4.24 & 0 \\
\hline & 4 & 3 & 0 & 13.87 & 0 \\
\hline & 4 & 7 & 0 & 8.31 & 0 \\
\hline & 4 & 9 & 0 & 8.94 & 0 \\
\hline & 5 & 1 & 0 & 5.17 & 0 \\
\hline & 5 & 4 & 0 & 10.75 & 0 \\
\hline & 5 & 6 & 0 & 1 & 0 \\
\hline \multirow{17}{*}{ Imidacloprid } & 1 & 1 & 1 & 2.64 & 75.76 \\
\hline & 1 & 2 & LOQ & 1.95 & - \\
\hline & 1 & 4 & 0 & 4 & 0 \\
\hline & 1 & 6 & 0 & 5.59 & 0 \\
\hline & 1 & 7 & 0 & 5.33 & 0 \\
\hline & 1 & 9 & 0 & 2.72 & 0 \\
\hline & 2 & 2 & 0 & 1.17 & 0 \\
\hline & 2 & 3 & 0 & 0.68 & 0 \\
\hline & 3 & 6 & 1.6 & 6.62 & 48.34 \\
\hline & 3 & 9 & 2 & 2.05 & 97.56 \\
\hline & 4 & 2 & 0 & 10.42 & 0 \\
\hline & 4 & 6 & 0 & 4.38 & 0 \\
\hline & 4 & 7 & 2.2 & 5.53 & 79.57 \\
\hline & 5 & 2 & 0 & 1.18 & 0 \\
\hline & 5 & 6 & LOQ & 7.31 & - \\
\hline & 5 & 7 & 1.1 & 10.14 & 21.70 \\
\hline & 5 & 7 & LOQ & 4.32 & 0 \\
\hline \multirow{14}{*}{ Thiamethoxam } & 1 & 3 & 0 & 1.72 & 0 \\
\hline & 1 & 4 & 0 & 2.59 & 0 \\
\hline & 1 & 6 & 0 & 0.86 & 0 \\
\hline & 1 & 9 & LOQ & 4.91 & - \\
\hline & 2 & 2 & 1.8 & 1.24 & 290.32 \\
\hline & 2 & 3 & 0 & 2.37 & 0 \\
\hline & 2 & 4 & LOQ & 0.51 & - \\
\hline & 2 & 5 & 0 & 1.41 & 0 \\
\hline & 2 & 9 & 0 & 1.26 & 0 \\
\hline & 3 & 2 & 0 & 0.33 & 0 \\
\hline & 3 & 9 & 0 & 6.93 & 0 \\
\hline & 4 & 4 & 0 & 1.3 & 0 \\
\hline & 5 & 4 & LOQ & 1.22 & - \\
\hline & 5 & 6 & LOQ & 6.19 & - \\
\hline
\end{tabular}

${ }^{*}$ LOQ: Limit of quantification 
Table S6 | LC-MS/MS retention time (TR), precursor ion $\rightarrow$ product ion transitions selected for selected reaction monitoring (SRM), fragmentor (Frag) and collision energy (CE) used to determine each insecticide.

\begin{tabular}{lcccccccc}
\hline Compound & $\mathrm{T}_{\mathrm{R}}$ & SRMI $^{*}$ & $\begin{array}{c}\text { Frag } \\
(\mathrm{eV})\end{array}$ & $\begin{array}{c}\mathrm{CE} \\
(\mathrm{eV})\end{array}$ & $\mathrm{SMR2}^{* *}$ & $\begin{array}{c}\text { Frag } \\
(\mathrm{eV})\end{array}$ & $\begin{array}{c}\mathrm{CE} \\
(\mathrm{eV})\end{array}$ & $\begin{array}{c}\text { SRM2/S } \\
\mathrm{RMI} \%\end{array}$ \\
\hline Thiamethoxam & 1.47 & $292 \rightarrow 211$ & 78 & 10 & $292 \rightarrow 132$ & 78 & 10 & 24.6 \\
Imidacloprid & 1.49 & $256 \rightarrow 209$ & 80 & 10 & $256 \rightarrow 175$ & 80 & 10 & 81.6 \\
Clothianidin & 1.52 & $250 \rightarrow 169$ & 86 & 5 & $250 \rightarrow 132$ & 86 & 9 & 60.7 \\
\hline
\end{tabular}

${ }^{*}$ SMR1: Precursor ion $\rightarrow$ product ion transitions used for quantitative purposes (calculation of peak area vs concentrations).

**SMR2: Precursor ion $\rightarrow$ product ion transitions used for quantitative purposes 


\section{Chapter 3}

\section{IPM-recommended insecticides harm beneficial insects through contaminated honeydew}

Miguel Calvo-Agudo, Joel González-Cabrera, Daniele Sadutto, Yolanda Picó, Alberto Urbaneja, Marcel Dicke, \& Alejandro Tena

Published in Environmental Pollution

https://doi.org/10.1016/j.envpol.2020.115581
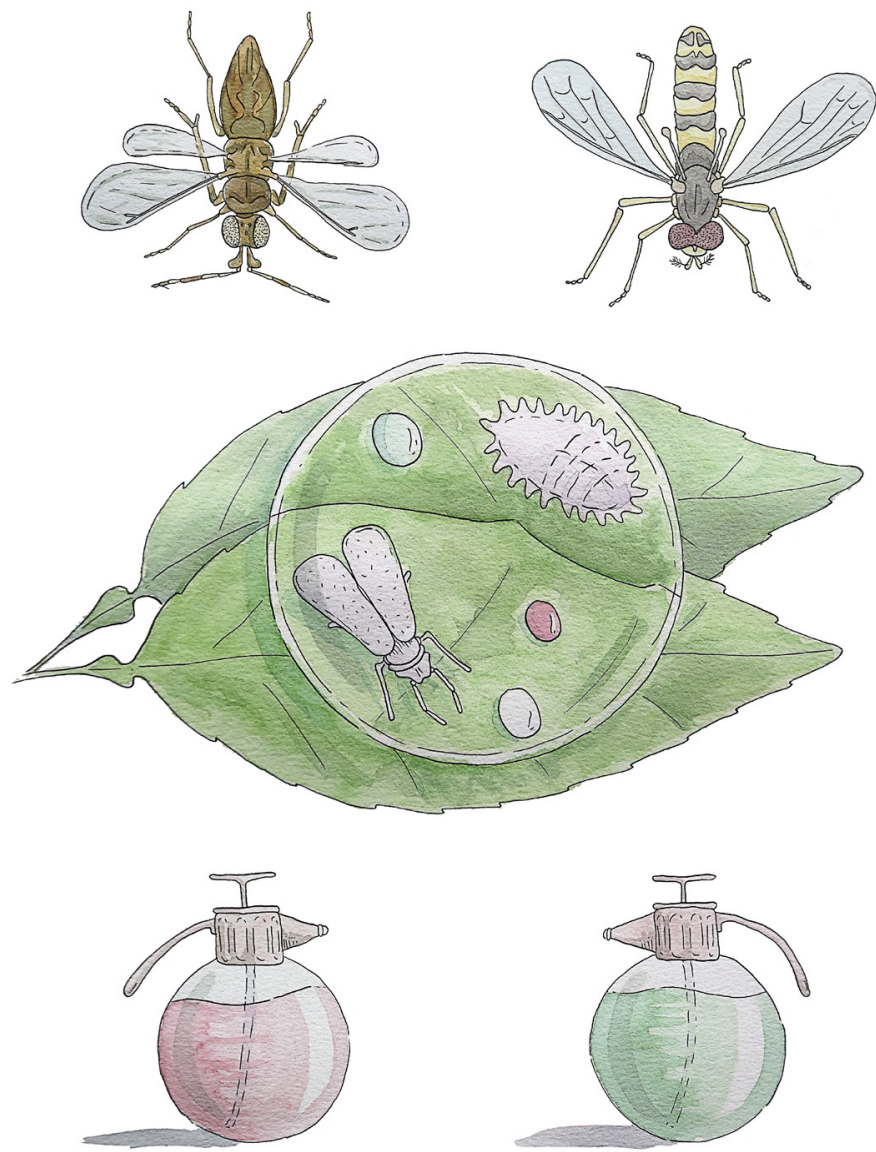



\section{ABSTRACT}

The use of some systemic insecticides has been banned in Europe because they are toxic to beneficial insects when these feed on nectar. A recent study shows that systemic insecticides can also kill beneficial insects when they feed on honeydew. Honeydew is the sugar-rich excretion of hemipterans and is the most abundant carbohydrate source for beneficial insects such as pollinators and biological control agents in agroecosystems. Here, we investigated whether the toxicity of contaminated honeydew depends on i) the hemipteran species that excretes the honeydew; ii) the active ingredient, and iii) the beneficial insect that feeds on it. HPLC-MS/MS analyses demonstrated that the systemic insecticides pymetrozine and flonicamid, which are commonly used in Integrated Pest Management programs, were present in honeydew excreted by the mealybug Planococcus citri. However, only pymetrozine was detected in honeydew excreted by the whitefly Aleurothixus floccosus. Toxicological studies demonstrated that honeydew excreted by mealybugs feeding on trees treated either with flonicamid or pymetrozine increased the mortality of the hoverfly Sphaerophoria rueppellii, but did not affect the parasitic wasp Anagyrus pseudococci. Honeydew contaminated with flonicamid was more toxic for the hoverfly than that contaminated with pymetrozine. Collectively, our data demonstrate that systemic insecticides commonly used in IPM programs can contaminate honeydew and kill beneficial insects that feed on it, with their toxicity being dependent on the active ingredient and hemipteran species that excretes the honeydew. 


\section{INTRODUCTION}

Systemic insecticides are widely used to manage insect pests in agriculture. However, these insecticides can impact non-target beneficial insects directly through contact when they are sprayed in crops, and indirectly through the food chain via cascading effects (Desneux et al., 2007; Kampfraath et al., 2017). One of the best-known routes of indirect exposure of systemic insecticides to beneficial insects is through the contamination of plant-derived food sources such as nectar and pollen (Bonmatin et al., 2015). For instance, the most widely used systemic insecticides, i.e. neonicotinoids, are well-known to reach these plant-derived food sources at concentrations ranging from 0.7 to $100 \mu \mathrm{g} /$ $\mathrm{kg}$ (Bonmatin et al., 2015; Whitehorn et al., 2012). A vast majority of beneficial insects, which provide ecosystem services like pollination or pest control (Losey and Vaughan, 2006), are highly dependent on these plant-derived food sources to support their daily physical activities and metabolic processes (Lundgren, 2009). As a consequence, a plethora of beneficial insects are exposed to lethal or sublethal concentrations of neonicotinoids when they feed on pollen and nectar. Their overuse has been considered one of the main stressors implicated in the decline of some pollinators (Henry et al., 2012; Sánchez-Bayo et al., 2017; Stapel et al., 2000; Tappert et al., 2017). For this reason, the use of several neonicotinoids was banned in Europe (European Food Safety Authority, 2018). However, there are other systemic insecticides that are still widely used. For example, the phloem-transported insecticides flonicamid and pymetrozine are applied against numerous pests in many crops. These insecticides have different modes of action, but ultimately both disrupt feeding and other behaviors in target insects (Belchim, 2020; Syngenta, 2020). Pymetrozine binds to and disrupts the gating properties of NanIav TRPV (Transient Receptor Potential Vanilloid) channel complexes in chordotonal stretch receptor organs. It induces, among other, neural inhibition of feeding behavior that eventually starves insects (Group 9B; Insecticide Resistance Action Committee, 2020). Flonicamid is also a modulator of the chordotonal organ function, but the specific site(s) responsible for its biological activity is still unknown. It is believed that it disturbs the insect feeding patterns (Group 29; Insecticide Resistance Action Committee, 2020). Both pymetrozine and flonicamid are considered selective and less toxic to beneficial insects than neonicotinoids. Therefore, they are recommended in different Integrated Pest Management (IPM) programs (Jansen et al., 2011).

Many ecotoxicological studies have evaluated the toxicity of these IPM-recommended insecticides on beneficial insects (Barbosa et al., 2018; Colomer et al., 2011; Jansen et al., 2011; Joseph et al., 2011; Moens et al., 2011; Tran et al., 2004). However, none of these studies have analysed a route of exposure that has been recently described, 
i.e. contaminated honeydew (Calvo-Agudo et al., 2019). Honeydew is the sugar-rich excretion product of hemipteran phloem feeders such as aphids, coccids, whiteflies, and psyllids that feed on crops, weeds or the surrounding natural vegetation (Heimpel and Jervis, 2005; Wäckers et al., 2005). Honeydew has typically been overlooked as a food source for beneficial insects because it was considered a carbohydrate source of poorer quality than nectar (Downes and Dahlem, 1987; Hagen, 1962; Lundgren, 2009; Wäckers et al., 2008). However, its quality as carbohydrate source for beneficial insects is variable and, due to its high degree of accessibility and abundance, it is the main carbohydrate source in most agroecosystems (Lundgren, 2009; Tena et al., 2016). In fact, honeydew is exploited by many beneficial insects including bees, hoverflies, ants, parasitic wasps and predators (Calabuig et al., 2015; Cameron et al., 2019; Hogervorst et al., 2007; Hölldobler and Wilson, 1990; Konrad et al., 2009; Lee et al., 2006; Tena et al., 2013b), likely because honeydew is more abundant than nectar and pollen in many agroecosystems (Lundgren, 2009; Tena et al., 2016; Wäckers et al., 2008).

It has been recently demonstrated that the systemic insecticides thiamethoxam, imidacloprid and spirotetramat are detected in honeydew excreted by hemipterans feeding on plants treated with these insecticides (Calvo-Agudo et al., 2019; Quesada et al., 2020). Furthermore, honeydew excreted by mealybugs feeding on trees treated with thiamethoxam or imidacloprid can be toxic for the pollinator and predator hoverfly Sphaerophoria rueppellii (Wiedemann) (Diptera: Syrphidae) and the parasitic wasp Anagyrus vladimiri (Girault) (Hymenoptera: Encyrtidae) (previously known as A. pseudococci) (Calvo-Agudo et al., 2019). Here, we explored whether the IPMrecommended insecticides pymetrozine and flonicamid: i) reach honeydew under controlled and field conditions when hemipterans feed on treated plants; ii) have lethal and/or sublethal effects on beneficial insects that feed on it; and iii) whether the excretion of insecticides differs between hemipteran species.

\section{MATERIALS AND METHODS}

\section{I. System}

We selected citrus as crop because numerous honeydew-producing species feed on citrus trees. Among the honeydew producers, the mealybug Planococcus citri (Risso) (Hemiptera: Pseudococcidae) was selected because: it is common in many citrus producing areas of the world although hardly ever reaching the economic injury level (Urbaneja et al., 2020); it excretes honeydew that increases the longevity and fecundity of beneficial insects (Tena et al., 2013a); and mealybugs are known to be tolerant to the insecticides pymetrozine and flonicamid (El-Zahi et al., 2016; Rezk et al., 2019). 
As beneficial insects, we selected the hoverfly S. rueppellii and the parasitic wasp A. vladimiri. Sphaerophoria rueppellii was selected because hoverflies are one of the most important groups of pollinators (Rader et al., 2015), their larvae feed on aphids and their populations are declining (Powney et al., 2019). Anagyrus vladimiri was selected because parasitic wasps represent one of the main groups of biological control agents in agriculture (Heimpel and Mills, 2017); it is the main biological control agent of $P$. citri and the genus Anagyrus represents one of the most successful examples used in biological control worldwide (Herren and Neuenschwander, 1991).

\subsection{Insects and experimental conditions}

The phloem-feeding herbivorous insect $P$. citri was obtained from the State Insectary of Generalitat Valenciana (Almassora, Spain), where it was reared on potato sprouts and transported to the Instituto Valenciano de Investigaciones Agrarias (IVIA) (Moncada, Spain) as crawlers (first instar) (Planes et al., 2013). The parasitic wasp A. vladimiri and the predator-pollinator $S$. rueppellii were obtained as pupae from the commercial companies Koppert Biological Systems S.L (Águilas, Spain) and Biobest Biological Systems (Westerlo, Belgium), respectively. Pupae were introduced into wooden and glass rearing boxes $(51 \times 51 \times 41 \mathrm{~cm})$ with holes in the wall that were covered with anti-aphid mesh. Rearing boxes were kept in the laboratory at room temperature until adults emerged. Unfed newly emerged parasitic wasps and hoverflies were collected daily between 9:00 and 11:00 AM and used in the experiments. All experiments were carried out in different climatic chambers for each insect species at $25 \pm 2{ }^{\circ} \mathrm{C}, 75 \pm 10 \% \mathrm{RH}$ and a photoperiod of $14: 10$ h (L:D).

\subsection{Plant infestation and insecticide application}

\subsection{Under controlled conditions}

Twenty-seven potted clementine trees cv. Clementina de Nules grafted on 'Macrophyla' (Citrus sinensis $\times$ Poncirus trifoliata) were grown in a greenhouse at IVIA until they were one-year-old and $\sim 1 \mathrm{~m}$ high. The environmental conditions in the greenhouse compartments were $22 \pm 5{ }^{\circ} \mathrm{C}, 70 \pm 20 \% \mathrm{RH}$ and natural photoperiod (February-April 2017). Clementine trees were watered three times per week and fertilized once per week with Sofertirrig ${ }^{\circledR}$ fertilizer (18-18-18 N-P-K). Plants were infested with $P$. citri crawlers on March 7, 2017. To infest them, $1.5 \mathrm{~mL}$ centrifuge tubes half-filled with $P$. citri crawlers were held on the crown of each plant (Calvo-Agudo et al., 2019). On 26 April 2017, we applied each insecticide or distilled water (control treatment) in separate chambers to nine clementine plants per treatment that we temporally removed from the greenhouse in order to prevent spray drift and cross-contamination of treatments. The insecticides 
used in this research were flonicamid [Flonicamid (50\%), Teppeki WG, Belchim)] and pymetrozine [(Pymetrozine (50\%), Plenum WG, Syngenta)]. Insecticides were sprayed at the dose recommended by the manufacturer. A concentration of $0.05 \mathrm{~g}$ of flonicamid / $\mathrm{L}$ of distilled water and a concentration of $0.4 \mathrm{~g}$ of pymetrozine / $\mathrm{L}$ of distilled water were applied on nine different plants per treatment. Water-treated trees (controls) were sprayed using only distilled water. We used $2 \mathrm{~L}$ manual sprayers and a different sprayer was used for each insecticide and the control. Insecticides were sprayed until run-off (200 $\mathrm{mL}$ per tree). One hour after spraying, the trees were returned to their previous positions in the greenhouse.

\subsubsection{Under field conditions}

Twelve 20-year-old untreated orange trees (Citrus sinensis) located at the Instituto Valenciano de Investigaciones Agrarias (UTM: 39³5'16.4”N 0²3'54.2”W) were selected and infested with $P$. citri crawlers on 20 August, 2018. Trees were approximately $2.5 \mathrm{~m}$ high. One twig per tree was infested. To infest the twigs, $1.5 \mathrm{~mL}$ centrifuge tubes half-filled with $P$. citri crawlers were held on the twig and covered individually with sleeve bags made from fine mesh organdy to allow ventilation and prevent $P$. citri crawlers from escaping. Mealybugs were kept undisturbed within the sleeve bags for 21 days. On 11 September 2018, we removed the exclusion bags and applied the insecticides flonicamid or pymetrozine or distilled water as control treatment. At this period of the year, the whitefly Aleurothrixus floccosus Maskell (Hemiptera: Aleyrodidae) had naturally infested all the selected trees. Two whitefly colonies were selected per tree in order to determine the presence of insecticides in A. floccosus honeydew. Whitefly colonies were settled on developed leaves and had more than 100 nymphs of different instars. The insecticides flonicamid and pymetrozine were applied onto the foliage at the dose recommended by the producer. Untreated controls were sprayed using only distilled water. Insecticides were applied until run-off using a wheelbarrow sprayer (Model ATASA MC-25) with a volume of about $5 \mathrm{~L}$ per tree.

\subsection{Honeydew collection}

\subsection{Under controlled conditions}

We collected fresh honeydew from the mealybug P. citri daily from 27 April 2017 (+1 day after treatment, DAT) to 2 May 2017 (+5DAT), by placing Parafilm ${ }^{\oplus}$ squares of $5 \mathrm{~cm} \times 5$ $\mathrm{cm}$ below the infested leaf during 24 hours. The collected honeydew for each treatment was labelled and stored at $-20^{\circ} \mathrm{C}$ in Petri dishes until samples were chemically analysed using HPLC-MS/MS or used in toxicity bioassays (Calvo-Agudo et al., 2019; Hogervorst et al., 2007; Tena et al., 2013b). The number of replicates per treatment, day and tree are provided in Tables 1 and 2. 


\subsubsection{Under field conditions}

Honeydew samples from the mealybug $P$. citri and the whitefly $A$. floccosus were collected on 14 September 2018 (+2DAT). Fresh honeydew was collected over a 24-h period by holding $10 \mathrm{~cm}$ wide and $17 \mathrm{~cm}$ long plastic punnets below each hemipteran colony. Within the punnets, two pieces of Parafilm ${ }^{\odot}$ were placed to collect the honeydew. To exclude ants from the samples, we used a wire coated with Tangle-trap (Tangle-foot; Biagro, Valencia, Spain) to hold the punnets. The collected honeydew for each treatment was labelled and stored at $-20^{\circ} \mathrm{C}$ in Petri dishes until they were used in the chemical analysis (Hogervorst et al., 2007; Tena et al., 2013b). Honeydew was labelled with information on the corresponding honeydew producer species, treatment, and tree number. The number of replicates per treatment, day and tree are provided in Tables 3 and 4.

\subsection{Chemical analysis of honeydew samples}

The presence and concentration of flonicamid and pymetrozine in the honeydew samples from both assays were further analysed using HPLC-MS/MS. Under controlled conditions, we collected honeydew samples excreted by the mealybug $P$. citri between +2 DAT and +5DAT. We used nine samples of honeydew excreted by mealybugs feeding on water-treated trees derived from six different trees; twelve samples of honeydew excreted by mealybugs feeding on trees treated with flonicamid derived from six trees; and fifteen samples of honeydew excreted by mealybugs feeding on trees treated with pymetrozine derived from nine trees (replicates per treatment and trees are provided in Tables 1 and 2).

Under field conditions, we collected honeydew samples excreted by the mealybug $P$. citri and the whitefly $A$. floccosus $+2 \mathrm{DAT}$. In total, after discarding some samples because of the small amount of honeydew collected, we analysed six samples of honeydew excreted by mealybugs feeding on water-treated trees derived from three trees; four samples of honeydew excreted by mealybugs feeding on trees treated with flonicamid from three trees; and seven samples of honeydew excreted by mealybugs feeding on trees treated with pymetrozine from four trees (replicates per treatment and trees are provided in Tables 3 and 4). For A. floccosus, we analysed eight samples of honeydew excreted by mealybugs feeding on water-treated trees derived from five trees; six samples of honeydew excreted by mealybugs feeding on trees treated with flonicamid from three trees and six samples of honeydew excreted by mealybugs feeding on trees treated with pymetrozine from three trees (Tables 3 and 4).

The numbers of honeydew droplets excreted by $P$. citri and A. floccosus were estimated following the methodology described by Calvo-Agudo et al., (2019). 


\subsection{Insecticide extraction from honeydew}

All honeydew droplets from the same honeydew producer species, same tree and day were dissolved in $200 \mu \mathrm{L}$ of $50 \%$ methanol. This diluent solution was deposited on top of the Parafilm ${ }^{\circledast}$ piece containing the honeydew droplets. The solution and the honeydew droplets were stirred gently with the same pipette to dissolve the honeydew and then filtered using acrodisc syringe filters of $13 \mathrm{~mm}$ with $0.2 \mu \mathrm{m}$ PTFE (Pall Corporation, New York, USA). Samples were drawn into $250 \mu \mathrm{L}$ propylene inserts (Agilent technologies) and subsequently frozen at $-20^{\circ} \mathrm{C}$ for HPLC-MS/MS analysis.

\subsection{Chemical analysis using HPLC-MS/MS}

The HPLC-MS/MS analysis was performed by using an infinity Ultra-High-performance Liquid Chromatography 1260 system coupled to Triple Quad Mass Spectrometry 6410 from Agilent Technologies (Santa Clara, CA, USA).

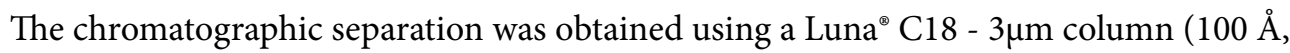
$150 \times 2.1 \mathrm{~mm}$; Phenomenex, Torrance, CA, USA). The analytical column temperature was kept at $25^{\circ} \mathrm{C}$ and the volume injected was $5 \mu \mathrm{L}$. The mobile phases were (A) Milli-Q water and (B) methanol, both with a $0.1 \%$ of formic acid. Working in isocratic conditions with an $80 \%$ of $\mathrm{A}$ and a $20 \%$ of $\mathrm{B}$. The flow rate was $0.3 \mathrm{~mL} \mathrm{~min}^{-1}$.

The ionization source was working in positive ionization mode (ESI+) with the following parameters: drying gas (nitrogen) flow of $11 \mathrm{~L} \mathrm{~min}^{-1}$ at $300{ }^{\circ} \mathrm{C}$, nebulizer pressure of 30 psi and capillarity voltage of $4000 \mathrm{~V}$. The Triple Quadrupole HPLC worked in SRM (selected reaction monitoring) mode. The MS/MS transitions were three for pymetrozine and two for flonicamid, as reported in detail in Table S1.

\subsection{Method validation and quality control.}

The calibration curve of the MS/MS analysis was performed using external standards dissolved in methanol, a concentration range of 2.5 to $25 \mathrm{ng} \mathrm{mL}^{-1}$ (six points) achieved by weighted least squares linear regression model $\left(1 / \mathrm{x}^{2}\right)$. Each curve was obtained by two independent injections. The calibration curves have coefficients of determination $\left(\mathrm{R}^{2}\right)>$ 0.99. The chromatograms were acquired and processed by Qualitative and Quantitative Mass Hunter Analysis software (Version 10.0) supplied by Agilent Technologies. Figures S1-S4 show several chromatograms that illustrate the method's performance. The limit of quantification (LOQ) and the limit of detection (LOD) were established as minimum concentrations of the analyte that can be the detected in spiked samples with $\mathrm{S} / \mathrm{N}$ (signal-to-noise), for the quantifier transition, $\geq 3$ for LOD and $\geq 10$ for LOQ (with 
the other transitions visible). LOD values were $0.007 \mathrm{ng} \mathrm{g}^{-1}$ for flonicamid, $0.660 \mathrm{ng} \mathrm{g}^{-1}$ for pymetrozine and LOQ values were $0.020 \mathrm{ng} \mathrm{g}^{-1}$ for flonicamid and $2.000 \mathrm{ng} \mathrm{g}^{-1}$ for pymetrozine.

\subsection{Mortality of beneficial insects}

Anagyrus vladimiri and S. rueppellii were fed on honeydew excreted by $P$. citri feeding on trees that had been sprayed three days before with flonicamid, pymetrozine or distilled water (control) under controlled conditions. For S. rueppellii, we individually confined newly emerged and unfed adults in 5.3-cm-diameter Petri dishes with 3 -cm-diameter holes covered with muslin mesh to allow ventilation. Thirty replicates per treatment were carried out (Calvo-Agudo et al., 2019). For A. vladimiri, we used groups of ten newly emerged and unfed females. These females were grouped in 5.3-cm-diameter Petri dishes with 3-cm-diameter holes covered with muslin mesh to allow ventilation. Ten replicates per treatment were carried out (100 individuals per treatment). Parafilm ${ }^{\oplus}$ pieces with honeydew of each treatment were defrosted and observed under the binocular to check for the presence of honeydew. Honeydew was administered ad libitum and renewed daily to avoid crystallization (Hogervorst et al., 2007). To ensure that honeydew had been provided ad libitum, the presence of honeydew on the Parafilm ${ }^{\odot}$ removed was checked after the renewal to assess that not all honeydew had been consumed. A piece of wet cotton wool was also placed and renewed daily to provide sufficient moisture. Petri dishes containing the beneficial insects were kept undisturbed in the climatic chambers for 72 hours and afterwards mortality was assessed.

\subsection{Sublethal effects on beneficial insects}

\subsection{Parasitic wasp longevity}

After 72 hours, between one and seven surviving females per replicate of the mortality experiment explained above were placed individually into glass vials (subreplicates) of $3 \mathrm{~cm}$ high and $0.8 \mathrm{~cm}$ diameter covered with wet cotton wool (number of individuals per replicate in Table S2). Parafilm ${ }^{\circledR}$ pieces with honeydew of each treatment were: defrosted, checked for the presence of honeydew; cut into pieces of different sizes (ca. $1.5-3 \mathrm{~cm}^{2}$ depending on the quantity of honeydew on each piece of Parafilm ${ }^{\circledast}$ ) to provide honeydew ad libitum (Calvo-Agudo et al., 2019); and placed in the glass vials. Diets were administered daily for each treatment and survival was checked. Glass vials with parasitic wasps were kept in a climate chamber until all individuals had died. Each surviving female was used as replicate because there were no significant differences between replicates (females coming from the same Petri dish) in any treatment. Therefore, we analysed 58 parasitic wasp individuals fed on honeydew from mealybugs feeding on trees treated 
with distilled water only, 56 on honeydew from mealybugs feeding on trees treated with flonicamid and 52 with pymetrozine.

\subsubsection{Parasitism and encapsulation}

After 72 hours, two or three surviving females per replicate were individually placed in 5.3-cm-diameter Petri dishes (subreplicates) with 3-cm-diameter holes covered with muslin mesh to allow ventilation (number of individuals per replicate in Table S3). Parafilm ${ }^{\oplus}$ pieces with honeydew of each treatment were: defrosted; checked for the presence of honeydew; cut into pieces of different sizes $\left(\mathrm{ca} .1 .5-3 \mathrm{~cm}^{2}\right.$ ) to provide honeydew ad libitum (Calvo-Agudo et al., 2019); and placed in the Petri dishes. Petri dishes also contained a piece of wet cotton wool, one A. vladimiri male previously fed on honey to allow mating and five third-instar $P$. citri hosts settled on a green bean. One day later, parasitic wasps were removed and the Petri dishes were kept in the climatic chamber for seven days. Then, the number of mummified (successful parasitism), dead and live mealybugs were counted. Live mealybugs were dissected on a drop of deionized water using entomological needles and scalpels under a stereo microscope to check for encapsulated eggs. We analysed the number of parasitized mealybugs (mummified and alive with encapsulated eggs) and encapsulation for: 27 parasitic wasp individuals fed on honeydew from mealybugs feeding on trees treated with distilled water only, 26 on honeydew from mealybugs feeding on trees treated with flonicamid and 27 with pymetrozine.

\subsection{Data analysis}

To analyse the mortality of the parasitic wasp and the hoverfly after feeding on honeydew for three days (lethal effect), we used a generalized linear model with quasi-binomial distribution. The mortality of the parasitic wasps was calculated as the number of dead parasitic wasps divided by total number of parasitic wasps per Petri dish. In both analyses, honeydew type was the explanatory variable and mortality the dependent variable. A Bonferroni post-hoc test using the "multcomp" package enabled pairwise comparisons between honeydew treatments.

We used different approaches to analyse sublethal effects of both insecticides present in the honeydew on the parasitic wasp: survivorship, number of parasitized mealybugs and encapsulation rate. The effect of the honeydew treatments on the survival of the parasitic wasp was represented by Kaplan-Meier survivorship curves and analysed by a Cox's Proportional Hazards model using the survival functions of the "survival" package. For this, we first checked that there were no significant differences between replicates 
(females coming from the same Petri dish used in mortality assays) in any treatment using Cox's Proportional Hazards models [Survivorship of parasitic wasp females fed on honeydew excreted by mealybugs feeding on trees treated with: water $\left(\chi_{9}^{2}=10.44, P\right.$ $=0.3)$, flonicamid $\left(\chi_{9}^{2}=14.76, P=0.1\right)$ or pymetrozine $\left(\chi_{7}^{2}=10.1, P=0.3\right)$ (number of individuals per replicate in Table S2)]. Then, we used each female as replicate. Parasitism was calculated by summing the number of successfully parasitized hosts, dead hosts with encapsulated eggs and alive hosts with encapsulated eggs divided by the total number of hosts:

$$
\text { Parasitism }=\frac{\begin{array}{c}
\text { number of successfully parasitized hosts + } \\
\text { dead hosts with encapsulated eggs }+ \\
\text { alive hosts with encapsulated eggs }
\end{array}}{\text { Total number of hosts }}
$$

Encapsulation was calculated by summing the number of dead hosts with encapsulated eggs and live hosts with encapsulated eggs and divided by the number of parasitized hosts:

$$
\text { Encapsulation }=\frac{\begin{array}{c}
\text { number of dead hosts with encapsulated eggs }+ \\
\text { live hosts with encapsulated eggs }
\end{array}}{\text { Number of parasitized hosts }}
$$

Both sublethal effects were then statistically analysed using a generalized linear mixed model with treatment as explanatory factor and replicate (parasitic wasps from the same Petri dish) as random factor using the "glmer" package. We assumed Poisson and binomial distributions for the number of eggs parasitized and encapsulation rates, respectively. All tests performed were analysed using $\mathrm{R}$ (version 3.3.2 for Mackintosh).

\section{RESULTS}

\section{I. Detection and quantification of insecticides under controlled conditions}

Under controlled conditions, flonicamid was detected in mealybug-produced honeydew from five out of the six trees treated with this insecticide and in $69.2 \%$ of the samples from these six trees (Table 1). These contaminated samples contained $215.8 \pm 52.3 \mathrm{ng}$ of flonicamid/mL of honeydew (ppb). No flonicamid was detected in honeydew produced by mealybugs feeding on water-treated trees.

Pymetrozine was detected in mealybug-produced honeydew from six out of the nine trees treated with this insecticide and in $60 \%$ of the samples from these nine trees (Table 2). These contaminated samples contained $37 \pm 12.1 \mathrm{ng}$ of pymetrozine/mL of honeydew (ppb). Pymetrozine was detected in one sample out of the nine samples analysed from the six control trees at a concentration of $56 \mathrm{ng}$ of pymetrozine/ $\mathrm{mL}$ of honeydew (ppb). 
Table 1 | Insecticide detection and quantification on honeydew excreted by the mealybug Planococcus citri feeding on water-treated trees or trees treated with flonicamid between +2 DAT and +5 DAT, under controlled conditions.

\begin{tabular}{lcccc}
\hline Treatment & Tree & $\begin{array}{c}\text { Number of samples } \\
\text { per tree between } \\
\text { +2DAT and +5DAT }\end{array}$ & $\begin{array}{c}\text { Number of samples } \\
\text { in which flonicamid } \\
\text { was detected }\end{array}$ & $\begin{array}{c}\text { Mean concentration } \\
\text { of flonicamid in the } \\
\text { tree }(\mathrm{ppb})^{*}\end{array}$ \\
\hline \multirow{5}{*}{ Control } & 1 & 2 & 0 & 0 \\
& 2 & 1 & 0 & 0 \\
& 3 & 1 & 0 & 0 \\
& 4 & 2 & 0 & 0 \\
& 5 & 1 & 0 & 0 \\
Flonicamid & 6 & 2 & 0 & 0 \\
& 1 & 2 & 1 & $64.7 \pm 64.7$ \\
& 2 & 3 & 3 & $355.9 \pm 110.3$ \\
& 4 & 4 & 3 & $95.1 \pm 50.2$ \\
& 5 & 2 & 1 & $29.5 \pm 29.5$ \\
\hline
\end{tabular}

*calculated as the mean \pm SE concentration for each tree.

Table 2 | Insecticide detection and quantification on honeydew excreted by the mealybug Planococcus citri feeding on water-treated trees or trees treated with pymetrozine between +2 DAT and +5 DAT, under controlled conditions.

\begin{tabular}{|c|c|c|c|c|}
\hline Treatment & Tree & $\begin{array}{l}\text { Number of samples } \\
\text { per tree between } \\
+2 \mathrm{DAT} \text { and }+5 \mathrm{DAT}\end{array}$ & $\begin{array}{c}\text { Number of samples } \\
\text { in which pymetrozine } \\
\text { was detected }\end{array}$ & $\begin{array}{l}\text { Mean concentration } \\
\text { of pymetrozine in } \\
\text { the tree (ppb) }\end{array}$ \\
\hline \multirow{6}{*}{ Control } & 1 & 2 & 1 & $28 \pm 28$ \\
\hline & 2 & 1 & 0 & 0 \\
\hline & 3 & 1 & 0 & 0 \\
\hline & 4 & 2 & 0 & 0 \\
\hline & 5 & 1 & 0 & 0 \\
\hline & 6 & 2 & 0 & 0 \\
\hline \multirow{9}{*}{ Pymetrozine } & 1 & 3 & 2 & $10.6 \pm 7.1$ \\
\hline & 2 & 2 & 0 & 0 \\
\hline & 3 & 2 & 2 & $33.5 \pm 0.5$ \\
\hline & 4 & 1 & 1 & 6.1 \\
\hline & 5 & 1 & 1 & 33 \\
\hline & 6 & 2 & 2 & $64.5 \pm 29.5$ \\
\hline & 7 & 1 & 0 & 0 \\
\hline & 8 & 2 & 0 & 0 \\
\hline & 9 & 1 & 1 & 1.4 \\
\hline
\end{tabular}

* calculated as the average concentration for each tree. 
3.2. Detection and quantification of insecticides in honeydew excreted by two species of honeydew producers under field conditions.

Two days after insecticide application in the field, flonicamid was detected in mealybugproduced honeydew from two out of the three trees treated (Table 3). These contaminated samples contained $30.1 \pm 5.6 \mathrm{ng}$ of flonicamid $/ \mathrm{mL}$ of honeydew (ppb). In contrast, no flonicamid was detected in honeydew excreted by the whitefly A. floccosus.

Table 3 | Insecticide detection and quantification of honeydew excreted by the mealybug Planococcus citri and the whitefly Aleurothrixus floccosus feeding on water-treated trees or trees treated with flonicamid under field conditions.

\begin{tabular}{|c|c|c|c|}
\hline $\begin{array}{l}\text { Honeydew } \\
\text { producer }\end{array}$ & Treatment & Tree & Concentration of flonicamid +2DAT (ppb) \\
\hline \multirow{6}{*}{ Planococcus citri } & \multirow{3}{*}{ Control } & 1 & 0 \\
\hline & & 2 & 0 \\
\hline & & 3 & 0 \\
\hline & \multirow{3}{*}{ Flonicamid } & 1 & 35.7 \\
\hline & & 2 & 24.5 \\
\hline & & 3 & 0 \\
\hline \multirow{7}{*}{$\begin{array}{l}\text { Aleurothrixus } \\
\text { floccosus }\end{array}$} & \multirow{4}{*}{ Control } & 1 & 0 \\
\hline & & 2 & 0 \\
\hline & & 3 & 0 \\
\hline & & 4 & 0 \\
\hline & \multirow{3}{*}{ Flonicamid } & 1 & 0 \\
\hline & & 2 & 0 \\
\hline & & 3 & 0 \\
\hline
\end{tabular}

Two days after insecticide application in the field, pymetrozine was detected in mealybug-produced honeydew from three out of the four trees treated (Table 4). These contaminated samples contained $93.6 \pm 50.3 \mathrm{ng}$ of pymetrozine $/ \mathrm{mL}$ of honeydew (ppb). Pymetrozine was also detected in whitefly-produced honeydew from all trees treated with this insecticide (Table 4). These contaminated samples contained 118.4 $\pm 48.4 \mathrm{ng}$ of pymetrozine $/ \mathrm{mL}$ of honeydew (ppb). Pymetrozine was detected in one tree out of the four control trees at a concentration of $9.7 \mathrm{ng}$ of pymetrozine/mL of honeydew (ppb). 
Table 4 | Insecticide detection and quantification of honeydew excreted by the mealybug Planococcus citri and the whitefly Aleurothrixus floccosus feeding on water-treated trees or trees treated with pymetrozine under field conditions.

\begin{tabular}{|c|c|c|c|}
\hline $\begin{array}{l}\text { Honeydew } \\
\text { producer }\end{array}$ & Treatment & Tree & Concentration of pymetrozine +2DAT (ppb) \\
\hline \multirow{7}{*}{ Planococcus citri } & \multirow{3}{*}{ Control } & 1 & 0 \\
\hline & & 2 & 0 \\
\hline & & 3 & 0 \\
\hline & \multirow{4}{*}{ Pymetrozine } & 1 & 0 \\
\hline & & 2 & 20.1 \\
\hline & & 3 & 189.8 \\
\hline & & 4 & 70.7 \\
\hline \multirow{7}{*}{$\begin{array}{l}\text { Aleurothrixus } \\
\text { floccosus }\end{array}$} & \multirow{4}{*}{ Control } & 1 & 0 \\
\hline & & 2 & 0 \\
\hline & & 3 & 0 \\
\hline & & 4 & 9.7 \\
\hline & \multirow{3}{*}{ Pymetrozine } & 1 & 27.2 \\
\hline & & 2 & 192.2 \\
\hline & & 3 & 135.7 \\
\hline
\end{tabular}

\subsection{Mortality of beneficial insects.}

All S. rueppellii hoverflies survived after three days feeding on honeydew excreted by mealybugs feeding on water-treated trees. In contrast, $56 \pm 10 \%$ of the hoverflies died in the flonicamid treatment and $22.2 \pm 8 \%$ in the pymetrozine treatment. Mortality siginficantly differed among the three treatments (GLM based on binomial distribution, $\chi_{2,53}=72.17, P<0.015$ ) (Figure 1.a).

Mortality of the parasitic wasp A. vladimiri was similar when it fed on honeydew excreted by mealybugs feeding on water-treated trees $(6.1 \pm 2.7 \%)$, trees treated with flonicamid $(11 \pm 4.8 \%)$ or pymetrozine ( $14 \pm 3.4 \%$ ) (GLM based on quasi-binomial distribution, $F_{2,27}$ $=1.21, P=0.31$ ) (Figure 1.b).

\subsection{Sublethal effects on parasitic wasps.}

The longevity of the surviving parasitic wasps was similar when feeding on honeydew excreted by mealybugs feeding on water-treated trees $(9.7 \pm 0.4$ days $)$, trees treated with flonicamid ( 8.7 \pm 0.4 ) or pymetrozine $(9 \pm 0.4)$ (Cox's Proportional Hazards: $\chi_{2}^{2}=1.97, P=0.37$ ) (Figure 2).

After feeding on honeydew for three days, parasitic wasps that fed on honeydew excreted by mealybugs feeding on trees treated with distilled water parasitized the same number 
of hosts ( $3.17 \pm 0.27$ parasitized mealybugs) as those fed on honeydew excreted by mealybugs feeding on trees treated with flonicamid ( $2.98 \pm 0.18$ parasitized mealybugs) or pymetrozine ( $3.47 \pm 0.25$ parasitized mealybugs) (GLMM based on Poisson, $\chi_{2}^{2}=1.25$, $P=0.54)$. Among the parasitized hosts, the percentage of encapsulated eggs was similar for the three treatments (water: $52.6 \pm 5.1 \%$; flonicamid: $52.8 \pm 10.1 \%$; pymetrozine: 55.7 $\pm 3.9 \%$; GLMM based on binomial, $\chi_{2}^{2}=0.04, P=0.98$ ).

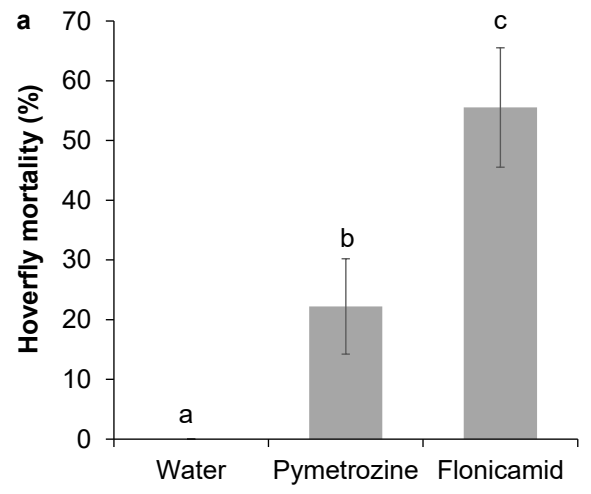

Source of honeydew

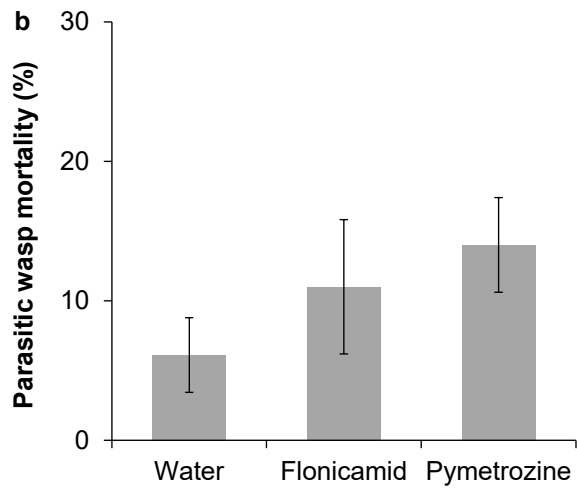

Source of honeydew

Figure 1 | Mortality (mean $\pm \mathrm{SE})$ of a) the parasitic wasp Anagyrus vladimiri $(\mathrm{N}=10$ replicates of 10 females each per treatment) and $\mathrm{b}$ ) the hoverfly Sphaerophoria rueppellii $(\mathrm{N}=30$ replicates per treatment) fed on honeydew of Planococcus citri feeding on water-treated trees or on honeydew of $P$. citri feeding on trees treated with the insecticides flonicamid or pymetrozine. Mortality was assessed after feeding on honeydew for 72 hours. Columns with different letters are significantly different from each other (GLM with quasibinomial distribution followed by a Bonferroni test, $P<0.05$ ).

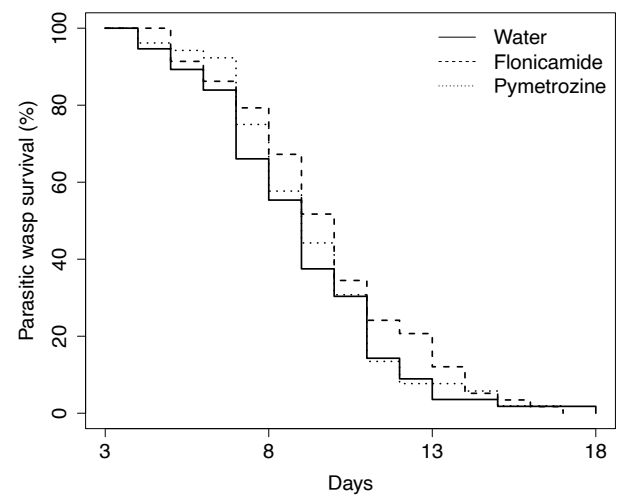

Figure 2 | Survival curve (mean \pm SE) estimated by Kaplan-Meier of the parasitic wasp Anagyrus vladimiri $\left(\mathrm{N}_{\text {Honeydew }}=58, \mathrm{~N}_{\text {Honeydew with flonicamid }}=56, \mathrm{~N}_{\text {Honeydew with pymetrozine }}=52\right)$ fed on honeydew of Planococcus citri feeding on water-treated trees or on honeydew of $P$. citri feeding on trees treated with the insecticides flonicamid or pymetrozine. 


\section{DISCUSSION}

Our results demonstrate that IPM-recommended insecticides, such as flonicamid and pymetrozine, reach honeydew at concentrations that can be toxic to beneficial insects. These insecticides were selected because they are phloem-transported and are applied in many crops including fruit trees, cereals, potatoes or vegetables to control numerous pests such as whiteflies, aphids, planthoppers or leafhoppers (Belchim, 2020; Syngenta, 2020). Flonicamid and pymetrozine are commonly foliar- or soil-applied. Here, we selected and tested the foliar application of both insecticides because it is the most common mode of application in citrus crops, as well as in other crops (Colomer et al., 2011; Qureshi et al., 2014). When sprayed, insecticides might reach honeydew through three different pathways: i) by contact with the honeydew producer - insecticides can be directly absorbed by the body of honeydew producers while it is sprayed, and honeydew producers excrete the insecticide via their honeydew; ii) through the plant - after spraying, systemic insecticides are translocated to all parts of the plant, the honeydew producer feeds on the plant and excretes the insecticide via its honeydew, and iii) insecticides can directly contaminate honeydew already present in crop. The third pathway can be excluded in our study because we did not collect honeydew just after the application. The collection started 48 hours after the insecticide application and the honeydew collected was excreted during the next 24 hours. The other two contamination routes cannot be differentiated and might occur simultaneously because both insecticides can act by contact and ingestion (Belchim, 2020; Syngenta, 2020).

Flonicamid and pymetrozine were detected in ca. $60-70 \%$ of the honeydew samples collected from the mealybug $P$. citri under controlled and field conditions. These results demonstrated that these insecticides are excreted by the mealybug under different conditions and at different times after their applications. In the field, where we collected honeydew excreted by the mealybug $P$. citri and the whitefly A. floccosus, flonicamid was detected in samples of honeydew excreted by the mealybug but not by the whitefly. This difference between hemipteran species might be explained by the different feeding behavior of honeydew producers and the physiochemical properties of flonicamid. Whiteflies such as $A$. floccosus feed mostly on plant phloem and stylets occasionally penetrate the xylem (Lei et al., 1997), whereas mealybugs such as $P$. citri feed frequently on both phloem and xylem (Obok et al., 2018). Therefore, A. floccosus is, compared to $P$. citri, less likely to excrete insecticides that move through the xylem. At $20^{\circ} \mathrm{C}$, flonicamid can move through xylem but, contrary to pymetrozine, does not have optimal phloem mobility and is less retained in the phloem sieve tubes (University of Herthfordshire, 2020; Bromilow et al., 1990). Overall, these results show that the presence and toxicity 
of insecticides via honeydew can vary not only among insecticides but also among honeydew-producing species. Therefore, further research that evaluates the presence of insecticides in honeydew should also take the honeydew-producing species into consideration.

Among honeydew-producing species, those that are tolerant or resistant to insecticides might excrete honeydew for a longer period of time. In our experiments, we used $P$. citri because it is tolerant to the insecticides pymetrozine and flonicamid (El-Zahi et al., 2016; Rezk et al., 2019). Since this mealybug is tolerant to these insecticides, it might excrete contaminated honeydew from a few days after the treatment, as occurred in our experiments, until these insecticides or their metabolites are completely degraded in the plant. The metabolites of flonicamid and pymetrozine can remain in citrus for more than 60 and 21 days after their application (Belchim, 2020; Syngenta, 2020). There are many other honeydew-producing species that are tolerant or resistant to insecticides. For example, the silverfleaf whitefly Bemisia tabaci (Gennadius) (Hemiptera: Aleyrodidae) and the green peach aphid Myzus persicae (Sulzer) (Hemiptera: Aphididae) feed on hundreds of plant species of more than forty families (Brown et al., 1995; Holman, 2009) and have developed resistance to more than 40 and 70 active ingredients, respectively (van Leeuwen et al., 2010), including pymetrozine (Gorman et al., 2010; Qiong et al., 2012). Therefore, the route of exposure described here, where tolerant/resistant hemipterans excrete contaminated honeydew, can be common in numerous crops.

The mortality of the hoverfly $S$. rueppellii was higher when it fed on honeydew contaminated by flonicamid than pymetrozine. However, honeydew contaminated with these insecticides was harmless to the parasitic wasp A. vladimiri. The hoverfly was also more susceptible than the parasitic wasp in our previous study with neonicotinoids (Calvo-Agudo et al., 2019). This difference between the two beneficial insects is likely because hoverflies are more sensitive to insecticides than parasitoids (Calvo-Agudo et al., 2019; Hallmann et al., 2017; Sanchez-Bayo, 2014), have a greater feeding rate (Cresswell et al., 2014), and/or a lower detoxification capacity (Manjon et al., 2018). Some studies have demonstrated lethal effect of flonicamid and pymetrozine, as well as a range of sublethal effects including a change in the feeding behavior, developmental period of nymphs, adult longevity, and fecundity of beneficial insects when these had been in contact with the insecticide residue (Jansen et al., 2011; Joseph et al., 2011; Moens et al., 2011). For instance, flonicamid increases the mortality of the parasitic wasp Aphidius rhopalosiphi (DeStefani-Perez) (Hymenoptera: Braconidae) (Jansen et al., 2011) and affects the reproductive performance (egg hatching and viable eggs per female) of the hoverfly 
Episyrphus balteatus (De Geer) (Diptera: Syrphidae) (Moens et al., 2011). Similarly, several studies have reported lethal and sublethal effects of pymetrozine. The parasitic wasp $A$. rhopalosiphi tends to die after contacting treated glass plates during 48 hours (Jansen et al., 2011) and the mortality of immature individuals of the hoverfly E. balteatus was also affected (Jansen et al., 2011). Sublethal effects include effects on Aphidius ervi Haliday (Hymenoptera: Braconidae) pre-imaginal development inside contaminated hosts (Joseph et al., 2011); male-biased sex ratio in A. ervi (Joseph et al., 2011); reduced host feeding in Neochrysocharis formosa (Westwood) (Hymenoptera: Eulophidae) (Tran et al., 2004); lower predation rate in Tenuisvalvae notata (Mulsant) (Coleoptera: Coccinellidae) larvae (Barbosa et al., 2018); or inability to discriminate between contaminated or uncontaminated hosts (Joseph et al., 2011). Most studies for both insecticides, however, did only consider toxicity through direct application or contact with residues. Only few studies took into account oral exposure through contaminated prey for predators (Colomer et al., 2011) or contaminated hosts for immature parasitoids (Joseph et al., 2011), but none explored the potential toxicity of contaminated carbohydrate sources such as floral and extrafloral nectar and honeydew. Therefore, to the best of our knowledge, this is the first study that considers oral toxicity of these insecticides in a carbohydrate source, although both insecticides are present not only in honeydew (presented here) but also in nectar and pollen (Azpiazu, 2020; Kyriakopoulou et al., 2017). Further studies should evaluate potential sublethal effects of pymetrozine and flonicamid on hoverflies when they feed on contaminated honeydew. In our study, flonicamid was more toxic than pymetrozine but these results are based on the lethal effects of these insecticides on $S$. rueppellii. As explained above, both insecticides can cause other detrimental effects that should be explored to evaluate the toxicity of these insecticides when hoverflies feed on carbohydrate sources contaminated with insecticides.

\section{CONCLUSION}

This study demonstrates that IPM-recommended insecticides such as pymetrozine and flonicamid may be present in honeydew excreted by hemipterans that are feeding on treated trees. We also show, for the first time, that the presence of insecticides in hemipteran honeydew depends on the hemipteran species. The results presented here, together with those of Calvo-Agudo et al. (2019) and Quesada et al. (2020) indicate that honeydew contaminated with insecticides can occur in many different agroecosystems. This route of exposure has been demonstrated for three species of honeydew producers belonging to three different families, five systemic insecticides with four different modes of action and translocation routes, and two plant species. Moreover, our results also suggest that honeydew-producing species that are tolerant or resistant to insecticides might excrete 
contaminated honeydew for longer periods. Therefore, contaminated honeydew is likely to affect a much wider range of beneficial insects than contaminated nectar and, thus, should be included in future environmental risk assessments.

\section{AKNOWLEDGMENTS}

We thank Biobest Biological Systems and Koppert Biological Systems for providing the beneficial arthropods used during the bioassays and the State Insectary of Generalitat Valenciana for providing P. citri. We acknowledge Dr. F.L. Wäckers for the recommendation to use the hoverfly in the assays. We also acknowledge P. Bru, J. Catalán, M. Montoro, A. Mouratidis, E. Rubio and P. Cuenca for their valuable help during the experiments. 


\section{SUPPORTING INFORMATION}

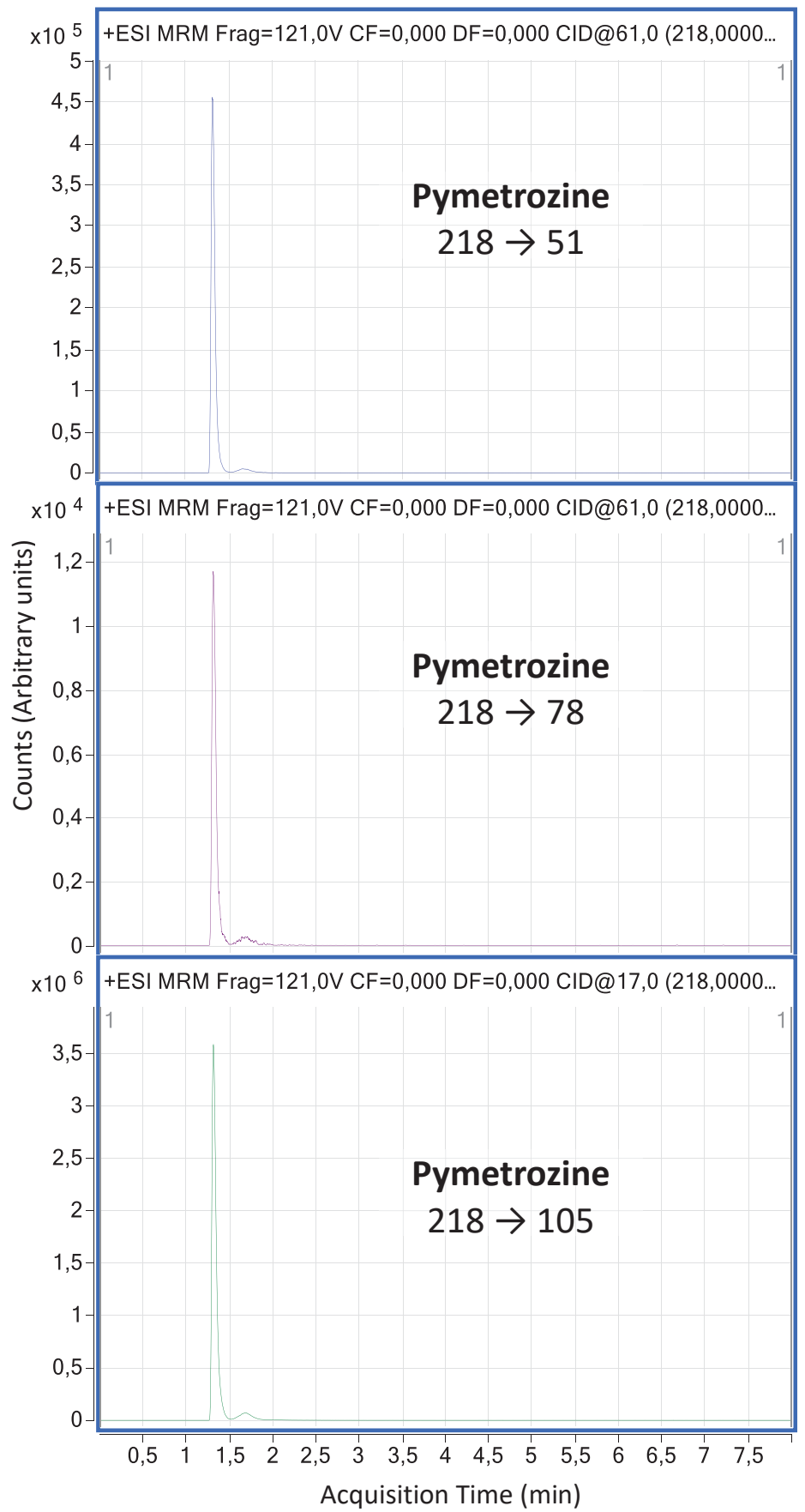

Figure S1 | LC-MS/MS chromatogram of an analytical standard is reported. It shows all multiple transitions (precursor ion $\rightarrow$ product ion transition) of pymetrozine at $25 \mathrm{ng} \mathrm{mL}^{-1}$. 


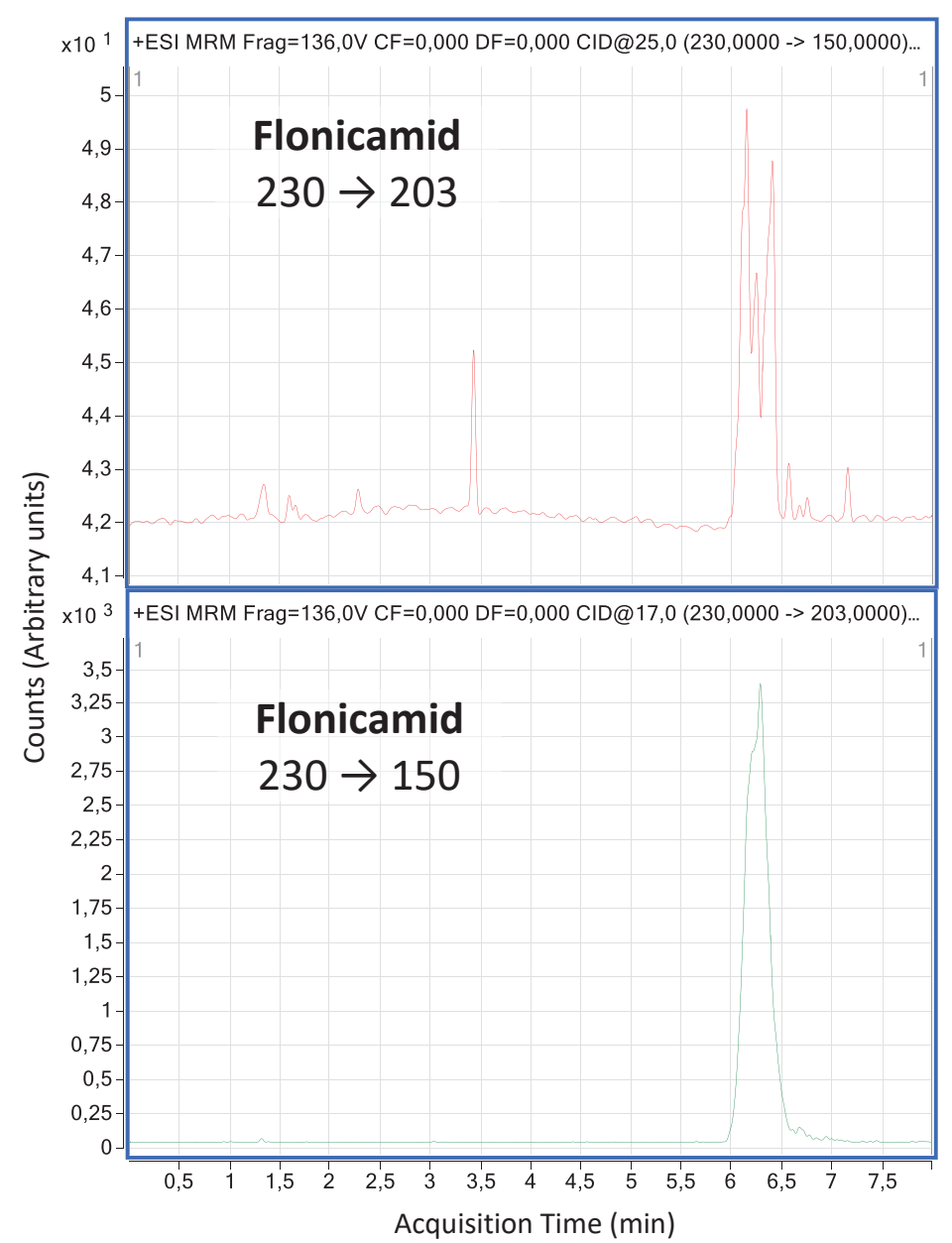

Figure S2 | LC-MS/MS chromatogram of an analytical standard is reported. It shows all multiple transitions (precursor ion $\rightarrow$ product ion transition) of flonicamid at $25 \mathrm{ng} \mathrm{mL}^{-1}$. 


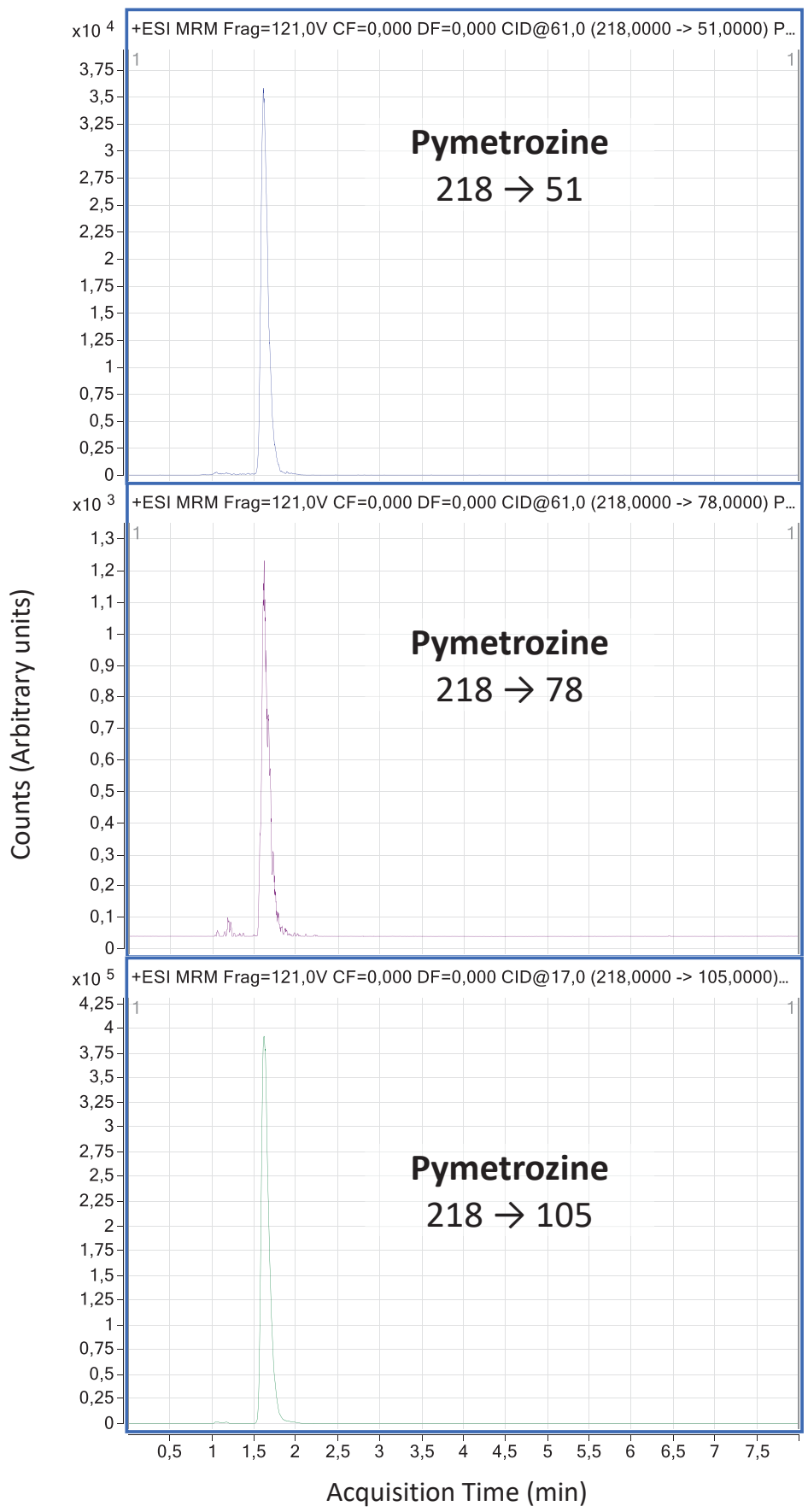

Figure S3 | LC-MS/MS chromatograms of pymetrozine in real sample is reported. It shows all multiple transitions (precursor ion $\rightarrow$ product ion transition) at $0.31 \mathrm{ng} \mathrm{mL}^{-1}$ in the final extract. 


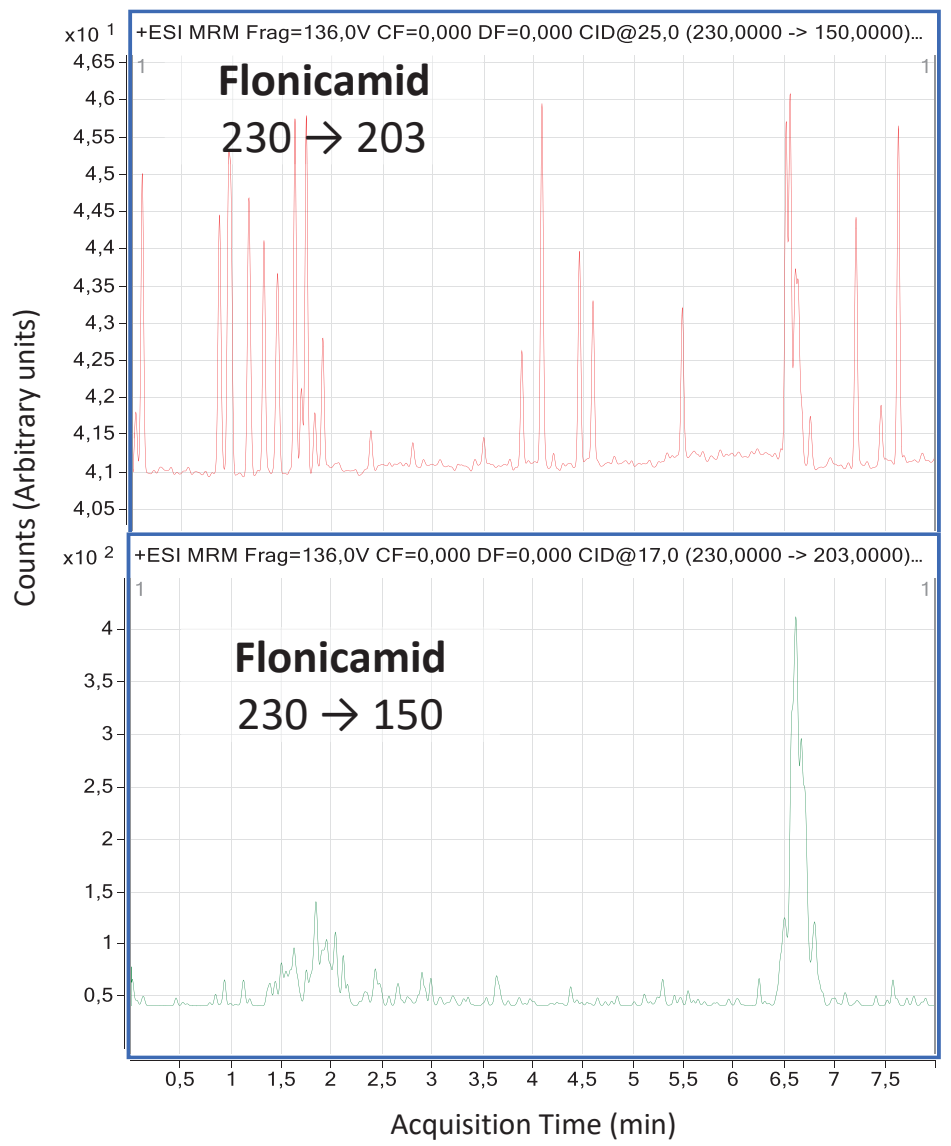

Figure S4 | LC-MS/MS chromatograms of flonicamid in real sample is reported. It shows all multiple transitions (precursor ion $\rightarrow$ product ion transition) at $4.30 \mathrm{ng} \mathrm{mL}^{-1}$ in the final extract. 
Table S1 | HPLC/MS-MS parameters, correlation coefficients $\left(\mathrm{R}^{2}\right)$, limit of detection (LOD) and limit of quantification (LOQ) of flonicamid and pymetrozine.

\begin{tabular}{|c|c|c|}
\hline & \multicolumn{2}{|c|}{ Compound Name } \\
\hline & Flonicamid & Pymetrozine \\
\hline Rt (min) & 6.45 & 1.31 \\
\hline Frag (V) & 136 & 121 \\
\hline SRM1 & $230>203$ & $218>105$ \\
\hline SRM2 & $230>150$ & $218>78$ \\
\hline SRM3 & 1 & $218>51$ \\
\hline $\mathrm{CE}_{1} / \mathrm{CE}_{2} / \mathrm{CE}_{3}(\mathrm{~V})$ & $17 / 25$ & $17 / 61 / 61$ \\
\hline Ratio \% ( \pm SD) & $8.1( \pm 1)$ & $0.2( \pm 0.1)$ \\
\hline \multicolumn{3}{|l|}{ (SRM1-SRM2) } \\
\hline $\mathbf{R}^{2}$ & 0.99 & 0.99 \\
\hline LOD (ng g $\left.\mathbf{g}^{-1}\right)$ & 0.020 & 0.007 \\
\hline LOQ (ng g g $\left.^{-1}\right)$ & 2.000 & 0.660 \\
\hline
\end{tabular}

Table S2 | Number of Anagyrus vladimiri used in the survival assay for each treatment and the initial replicate from where they were used.

\begin{tabular}{lccc}
\hline Replicate & $\begin{array}{c}\text { Control } \\
\text { honeydew }\end{array}$ & $\begin{array}{c}\text { Treatment } \\
\text { Honeydew with } \\
\text { flonicamid }\end{array}$ & $\begin{array}{c}\text { Honeydew with } \\
\text { Pymetrozine }\end{array}$ \\
\hline $\mathbf{1}$ & 6 & 5 & 5 \\
$\mathbf{2}$ & 6 & 6 & 4 \\
$\mathbf{3}$ & 3 & 5 & 6 \\
$\mathbf{4}$ & 6 & 4 & 4 \\
$\mathbf{5}$ & 7 & 7 & 6 \\
$\mathbf{6}$ & 5 & 7 & 7 \\
$\mathbf{7}$ & 6 & 6 & 5 \\
$\mathbf{8}$ & 7 & 3 & 7 \\
$\mathbf{9}$ & 5 & 6 & 4 \\
$\mathbf{1 0}$ & 7 & 7 & 10 \\
\hline Total & $\mathbf{5 8}$ & $\mathbf{5 6}$ & $\mathbf{5 2}$ \\
\hline
\end{tabular}


Table S3 | Number of Anagyrus vladimiri used in the parasitism and encapsulation assay for each treatment and the initial replicate from where they were used.

\begin{tabular}{lccc}
\hline Replicate & $\begin{array}{c}\text { Control } \\
\text { honeydew }\end{array}$ & $\begin{array}{c}\text { Treatment } \\
\text { Honeydew with } \\
\text { flonicamid }\end{array}$ & $\begin{array}{c}\text { Honeydew with } \\
\text { pymetrozine }\end{array}$ \\
\hline $\mathbf{1}$ & 3 & 3 & 3 \\
$\mathbf{2}$ & 3 & 3 & 3 \\
$\mathbf{3}$ & 3 & 3 & 3 \\
$\mathbf{4}$ & 3 & 3 & 3 \\
$\mathbf{5}$ & 3 & 3 & 3 \\
$\mathbf{6}$ & 2 & 2 & 2 \\
$\mathbf{7}$ & 2 & 2 & 2 \\
$\mathbf{8}$ & 2 & 2 & 2 \\
$\mathbf{9}$ & 3 & 3 & 3 \\
$\mathbf{1 0}$ & 3 & 2 & 3 \\
\hline Total & $\mathbf{2 7}$ & $\mathbf{2 6}$ & $\mathbf{2 7}$ \\
\hline
\end{tabular}




\section{Chapter 4}

\section{Neonicotinoids from coated seeds toxic for honeydew-feeding biological control agents}

Miguel Calvo-Agudo, Jonathan Dregni, Joel González-Cabrera,

Marcel Dicke, George E. Heimpel \& Alejandro Tena

Published in Environmental Pollution

https://doi.org/10.1016/j.envpol.2021.117813

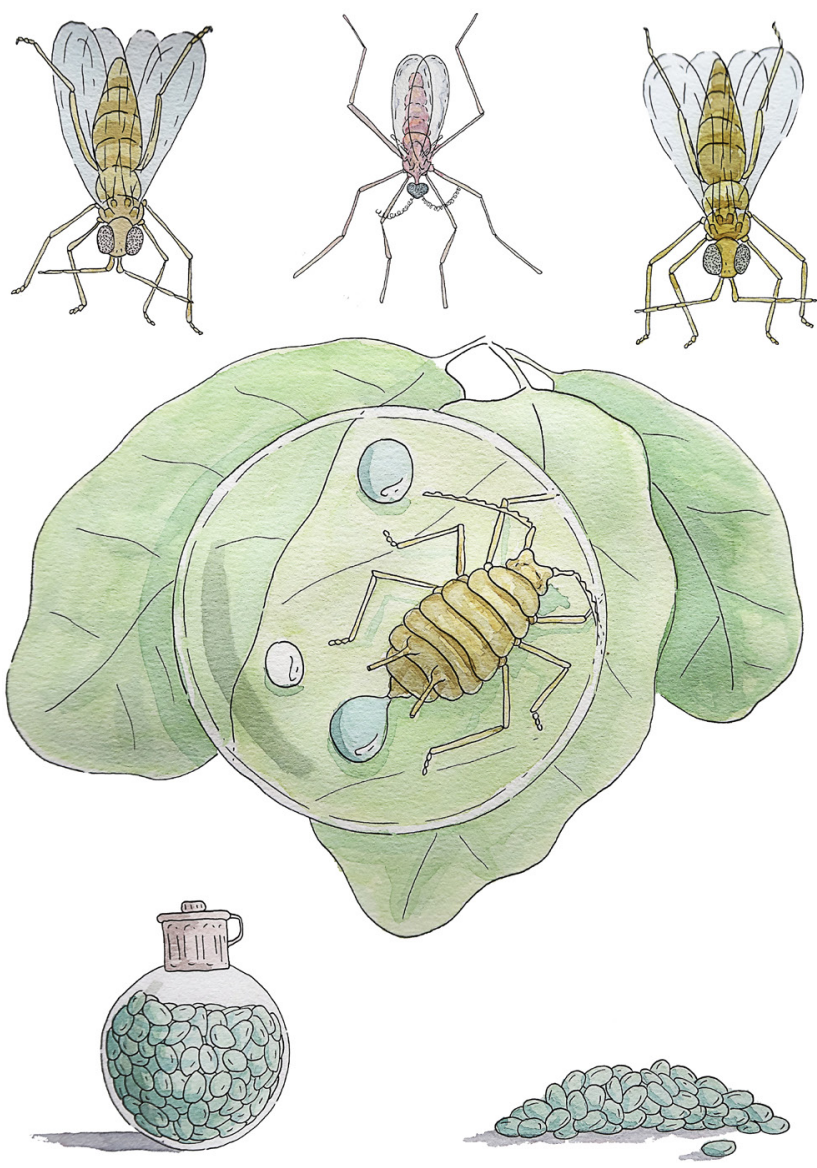





\section{ABSTRACT}

Seed coating ('seed treatment') is the leading delivery method of neonicotinoid insecticides in major crops such as soybean, wheat, cotton and maize. However, this prophylactic use of neonicotinoids is widely discussed from the standpoint of environmental costs. Growing soybean plants from neonicotinoid-coated seeds in field, we demonstrate that soybean aphids (Aphis glycines) survived the treatment, and excreted honeydew containing neonicotinoids. Biochemical analyses demonstrated that honeydew excreted by the soybean aphid contained substantial concentrations of neonicotinoids even one month after sowing of the crop. Consuming this honeydew reduced the longevity of two biological control agents of the soybean aphid, the predatory midge Aphidoletes aphidimyza and the parasitic wasp Aphelinus certus. These results have important environmental and economic implications because honeydew is the main carbohydrate source for many beneficial insects in agricultural landscapes. 


\section{INTRODUCTION}

Seed coating ('seed treatment') is the leading delivery method of neonicotinoid insecticides (Frank and Tooker, 2020; Matsuda et al., 2020). Seeds coated with neonicotinoids have been routinely used for major crops such as soybean, wheat, cotton and maize. For example, the seeds of over $50 \%$ of soybeans, $52-77 \%$ of cotton, and $79-100 \%$ of maize sown in the United States were coated with neonicotinoids in 2011 (Douglas and Tooker, 2015; Hurley and Mitchell, 2017), although they increased yield in less than 5\% of the cases (Labrie et al., 2020). Seeds coated with neonicotinoids have been extensively used over the last decades because they can be applied against a broad spectrum of insect pests at the beginning of the cropping cycle without the economic costs of spraying (Matsuda et al., 2020). However, evidence is growing for a causal link between the use of neonicotinoids and decreases in biomass and biodiversity of beneficial insects, including pollinators and biological control agents that regulate agricultural pests (Goulson, 2013; Krupke and Tooker, 2020; Woodcock et al., 2017). Neonicotinoids from coated seeds contaminate floral and extrafloral nectar because these pesticides are taken up systemically by the growing plant and distributed to all tissues (Goulson, 2013; Rundlöf et al., 2015; Whitehorn et al., 2012). Many beneficial insects therefore become exposed to neonicotinoids when they feed on contaminated nectar and pollen (Krischik et al., 2007; Rundlöf et al., 2015).

A recent study under controlled conditions identified a route of exposure of neonicotinoids to beneficial insects that can be more influential than nectar in extensive monocultures (Calvo-Agudo et al., 2019). In these landscapes, honeydew is often the main carbohydrate source for biological control agents because nectar is limited to the brief flowering period of the crop or to the presence of wild flowers or weeds growing in field margins (Lundgren, 2009; Tena et al., 2016). Honeydew is a nutritious and ubiquitous carbohydrate source excreted by hemipteran phloem-feeding insects such as aphids, whiteflies, mealybugs, coccids, and psyllids that feed on crops. Under controlled conditions, plants sprayed or watered with systemic insecticides including neonicotinoids can support hemipterans that survive the treatment and excrete contaminated honeydew that is toxic for biological control agents and pollinators (Calvo-Agudo et al., 2019). However, it remains unexplored whether this route of exposure is present in crops grown from neonicotinoid-coated seeds, which represents the main use of neonicotinoids worldwide, and thus has the potential to affect biological control agents at a large-scale worldwide ${ }^{1}$. In the United States alone, the ecosystem services provided by biological control agents have been conservatively estimated to be 4.5 billion dollars per year (Losey and Vaughan, 2006). 
Soybeans represent a major crop in the United States, with more than 35 million hectares planted in 2018 (FAOSTAT, 2021), and an estimated 85\% or more of it is sown with seeds coated with neonicotinoids combined with fungicides (Hurley and Mitchell, 2017). Neonicotinoid seed treatments are often combined with fungicides, which can synergize the toxic effect of the neonicotinoids in nectar on beneficial insects (Sgolastra et al., 2017). The main target pest of soybean grown from insecticide-coated seeds in the North-Central United States and Eastern Canada is the soybean aphid, Aphis glycines Matsumura (Hemiptera: Aphididae), an invasive pest first documented in North America in 2000 (Ragsdale et al., 2011). However, the usefulness of these seed treatments in controlling soybean aphid has been questioned (Krupke et al., 2017; Mourtzinis et al., 2019). The protection period of coated seeds lasts approximately 3-4 weeks after planting (Krupke et al., 2017; Mourtzinis et al., 2019; Seagraves and Lundgren, 2012), but the active ingredient remains in the plant for a longer period at lower concentrations (Krupke et al., 2017; Magalhaes et al., 2009). During this period of insecticide degradation, many aphids survive these sublethal concentrations (Krupke et al., 2017; Magalhaes et al., 2009; Mccornack and Ragsdale, 2006) and might excrete honeydew contaminated with neonicotinoids that harm biological control agents.

Soybean aphid honeydew represents the main carbohydrate source for many beneficial insects in soybean fields (Dieckhoff et al., 2014; Heimpel et al., 2004; Lee et al., 2006) and increases their fitness when they feed on it (Dieckhoff et al., 2014; Lee et al., 2006; Tena et al., 2018; Wyckhuys et al., 2008). Two groups of biological control agents that commonly feed on honeydew are predators and parasitic wasps of aphids. The aphid-feeding predatory midge Aphidoletes aphidimyza Rondani (Diptera: Cecidomyiidae) is the most abundant dipteran predator in soybean fields and uses honeydew as food source and kairomone (Boulanger et al., 2019; Kaiser et al., 2007). The parasitic wasp Aphelinus glycinis Hopper and Woolley (Hymenoptera: Aphelinidae) was purposefully introduced in United States to control the soybean aphid (Hopper et al., 2017), and Aphelinus certus Yasnosh (Hymenoptera: Aphelinidae) was accidentally introduced and is now abundant throughout soybean growing areas of North America (Frewin et al., 2010; Kaser and Heimpel, 2018; Miksanek, 2020; Miksanek and Heimpel, 2019). Importantly, Frewin et al. (2014) showed that $A$. certus is susceptible to the neonicotinoids thiamethoxam and imidacloprid presented as soybean seed treatments in laboratory studies, and suggested that such seed treatments may limit the effectiveness of this parasitoid as a biological control agent of soybean aphid.

Here, we aimed i) to determine whether the neonicotinoid thiamethoxam from coated seeds reaches honeydew excreted by the soybean aphid Aphis glycines in a soybean crop; 
ii) to evaluate the toxicity for three species of biological control agents of honeydew obtained from plants whose seeds had been coated with neonicotinoids; and iii) to determine whether the use of fungicides in coated seeds synergizes the toxic effects of neonicotinoids via honeydew.

\section{MATERIALS AND METHODS}

\section{I. Insect colonies}

The soybean aphid was reared under laboratory conditions at the University of Minnesota on soybean plants grown from uncoated seeds following the methodology of Desneux et al. (2009) and this colony was used to rear the parasitic wasps A. certus and A. glycinis. Parasitic wasp colonies were maintained by placing approximately twenty individual parasitic wasps onto two soybean plants infested with aphids inside plexiglass rearing cages $(30 \mathrm{x} 35 \mathrm{x}$ $40 \mathrm{~cm}$ ) with ventilation provided by three $10 \mathrm{~cm}$ diameter holes that were covered with fine mesh. Parasitic wasp colonies were initiated weekly to provide sufficient females for the entire experiment. Parasitized aphids containing pupae ('mummies') of either parasitic wasp species were placed individually into glass vials $(3 \mathrm{~cm}$ long $\mathrm{x} 0.8 \mathrm{~cm}$ diameter) plugged with cotton. Mummies were checked daily for emergence between 9:00 and 11:00 am and were sexed after emergence. Newly emerged females were used for the experiments.

The predatory midge was obtained from Koppert Biological Systems. Pupae were introduced into rearing cages of the type described above and kept in climatic chambers until emergence. All insects were kept separately by species in growth chambers at $25^{\circ} \mathrm{C}$, 65\% R.H. and 16:8 h L:D.

\subsection{Study site and experimental design}

The experiment was conducted at the University of Minnesota Agricultural Experiment Station, Saint Paul, U.S.A, between June and August 2019. The field experiment consisted of 24 soybean plots in a grid of 6 plots by 4 plots that were sown on June 19, 2019. Each plot was $2.5 \times 2.5 \mathrm{~m}$, and consisted of four soybean rows planted at a density of 35.6 seeds per $\mathrm{m}^{2}$ (355,831 seeds per ha). Rows within each plot were separated by $0.76 \mathrm{~m}$ and plots were separated by $7.25 \mathrm{~m}$. Buckwheat was planted and mown weekly between plots. Soybean and corn seeds coated with neonicotinoids were sowed in the same field the previous year, 2018.

We used a randomized complete block design of three different treatments each with eight replicates. Soybean seeds of the variety S14-B2X (Syngenta Crop Protection, USA) were uncoated or coated with either the insecticide thiamethoxam (Cruiser 
$5 \mathrm{FS}^{\oplus}$, Syngenta) at a concentration of $0.0756 \mathrm{mg}$ active ingredient per seed, or with the insecticide thiamethoxam in addition to the fungicides sedaxane, mefenoxam (also called R-metalaxyl), and fludioxonil (CruiserMaxx Vibrance ${ }^{\oplus}$, Syngenta) at a combined concentration of $0.0945 \mathrm{mg}$ per seed for all active ingredients per seed, of which 0.0756 mg was thiamethoxam.

\subsection{Plant infestation}

Soybean plants were infested with the soybean aphid on July 16, 2019 (27 days after $\underline{\text { sowing, }}$ DAS). For this, we placed infested leaves from a laboratory colony with approximately 50 A. glycines of different instars on approximately ten plants per plot. Two plants per plot that were infested in this way were subsequently covered with exclusion cages to protect the infested leaves from natural enemies (Kaser and Heimpel, 2018). Exclusion cages consisted of a wire frame cage of $85 \mathrm{~cm}$ tall, and $35 \mathrm{~cm}$ x $35 \mathrm{~cm}$ square and were covered with a fine mesh $(240 \mu \mathrm{m} \times 240 \mu \mathrm{m}$ gaps $)$.

\subsection{Honeydew collection}

Honeydew was collected in two temporal replicates. The first temporal replicate comprised honeydew collected on the following days: July 19, 2019 (+ 30 DAS), and every day from July 23 (+34 DAS) until July 26 (+37 DAS) inclusive. The second temporal replicate consisted of honeydew collected every day from July 30 (+41 DAS) to August $1(+43$ DAS) inclusive. To collect honeydew, Parafilm ${ }^{\circledast}$ squares of $10 \mathrm{~cm} \mathrm{x} 10 \mathrm{~cm}$ were placed singly inside 14-cm Petri dishes inside the exclusion cage, and the cover of the Petri dish was modified with a fine mesh for ventilation (Figure S1). A soybean leaf infested with 50-100 soybean aphids was inserted into the dish with the petiole passing through a hole in the side of the Petri dish in such a way that the infested leaf was suspended above the Parafilm ${ }^{\circledR}$ (Figure S1). The Parafilm ${ }^{\circledR}$ squares were left inside the Petri dishes in this manner for 24 hours and stored at $-20{ }^{\circ} \mathrm{C}$ until the honeydew was used in the bioassays described below.

\subsection{Concentration of thiamethoxam in honeydew}

We first estimated the amount of honeydew (i.e. the number of droplets) excreted by soybean aphids feeding on soybean plants following the methodology of Calvo-Agudo et al. (2019). The amount of honeydew produced by the soybean aphid per treatment, time replicate and plot was assessed by counting the total number of small (less than $150 \mu \mathrm{m} \varnothing$ ), medium (between 150 and $300 \mu \mathrm{m} \varnothing$ ), and large (more than $300 \mu \mathrm{m} \varnothing$ ) honeydew droplets on each Parafilm ${ }^{\odot}$ piece under a stereo microscope. The volume of each categorized droplet was estimated as $\left(4 / 3 \times \pi \times r^{3}\right) \times 1 / 2$, where $r$ is the radius of 
the droplet (Table S1). To ensure sufficient honeydew volume, we combined samples collected +30 DAS and +37 DAS for the first time replicate. For the second, we combined samples collected +41 DAS and +42 DAS. In total, we used honeydew samples from three treatments, seven to eight plots per treatment and from two time replicates to assess the presence of insecticide in the honeydew samples.

All droplets of honeydew from the same time replicate and plot were dissolved in 'Sample Diluent Buffer' (Imidacloprid ELISA, Microtiter Plate-kit, Abaraxis. Inc., Spain). Two hundred microliters of 'Sample Diluent Buffer' solution were pipetted onto the Parafilm ${ }^{\circledast}$ piece containing the honeydew droplets. The diluent solution and the honeydew droplets were stirred gently to dissolve the honeydew and then transferred into microcentrifuge tubes.

The presence and concentration of thiamethoxam in honeydew samples was estimated using an enzyme-linked immunosorbent assay for imidacloprid (ELISA-Imidacloprid, Microtiter Plate; Abraxis). This assay, although designed to detect imidacloprid, also detects clothianidin with $121 \%$ cross-reactivity, according to the manufacturer's specifications. Given that thiamethoxam is quickly metabolized to clothianidin in plants and insects and that the latter is the responsible for the insecticidal activity (Nauen et al., 2003; Tomizawa and Casida, 2005), we measured the presence and quantity of clothianidin in our samples as a proxy of that of thiamethoxam. All quantities were corrected considering the $121 \%$ cross-reactivity of clothianidin. This method allowed the quantification of very low amounts of insecticide, including potential residual contaminations from previous treatments in the experimental field (Masiá et al., 2013). In our assays, we detected the chemical in the samples coming from control treatments (average $0.13 \mathrm{ng} \mathrm{mL}^{-1}$; see Results). Hence, for the sake of accuracy, we corrected the values of all treatments by subtracting the average detection from the controls (CalvoAgudo et al., 2019; Masiá et al., 2013). Negative values after the correction were converted to zero (Calvo-Agudo et al., 2019; Masiá et al., 2013).

\subsection{Effect of honeydew on beneficial insects}

To determine the effects of seed treatments on the beneficial insects' longevity, we fed adults of the predatory midge and parasitoids of genus Aphelinus with honeydew excreted by the soybean aphid that had fed on plants whose seeds had been untreated or coated either with thiamethoxam or with thiamethoxam and fungicides. For the predatory midge, we confined between 40 and 42 newly emerged and unfed female adults individually in $6.1 \mathrm{~cm}$ high and $2.8 \mathrm{~cm}$ diameter vials covered with damp cotton for each 
of the three treatments for a total of 122 individuals. For the parasitic wasps, we placed between 38 and 41 newly emerged unfed females individually into $3 \mathrm{~cm}$ high and $0.8 \mathrm{~cm}$ diameter glass vials covered with damp cotton for each of the treatments for a total of 116 A. certus and 123 A. glycinis individuals. Honeydew from each plot was used to feed between two and eight individuals of each species.

Parafilm $^{\circledast}$ pieces with honeydew of each treatment were thawed, observed under a stereo microscope to check for the presence of honeydew and cut into pieces of different sizes to provide honeydew ad libitum (this was at least 10-15 and 25-30 droplets of different sizes for both parasitic wasp species and predator, respectively). Honeydew was renewed daily to avoid crystallization (Hogervorst et al., 2007). To confirm that all insects had received honeydew ad libitum, the presence of honeydew on the Parafilm ${ }^{\odot}$ piece was checked again when it was replaced. If there was no honeydew remaining on the Parafilm ${ }^{\otimes}$, the replicate was censored. Vials were kept in climatic chambers until the insects died and mortality was tabulated daily. The individuals from each treatment were fed on honeydew from six to eight plots of their corresponding treatments depending on the amount of honeydew available.

Climatic conditions for the predatory midge were $25^{\circ} \mathrm{C}, 80 \%$ relative humidity $(\mathrm{RH})$ and 16:8 h light:dark (L:D) and for A. certus and A. glycinis were $22^{\circ} \mathrm{C}, 80 \%$ relative humidity (RH) and 16:8 h light:dark (L:D)

\subsection{Statistical analysis}

To analyse the difference in the concentration of thiamethoxam in the honeydew samples, we used a generalized linear mixed model with gamma distribution. The field plot was included as a random factor and treatment and time replicate as fixed factors. Non-significant factors were excluded from the final model. A Tukey post hoc test using the "lsmeans" package in $\mathrm{R}$ enabled pairwise comparisons between the concentrations found in the honeydew treatments. The toxicity of each honeydew treatment on the beneficial insect's survivorship was represented by Kaplan-Meier survivorship curves and analysed by a Cox's Proportional Hazards for the predatory midge using the survival functions of the "Survival" package in $\mathrm{R}$ and by a log-rank test for both parasitic wasps. We censored those beneficial insects that escaped, died for other reasons, or had finished all the honeydew administered in one day (honeydew not ad libitum). For the predatory midge, we censored eighteen females out of 122 that escaped or died for other reasons and eleven out of 122 because they ran out of food during the trial. For A. certus, we censored nineteen females out of 116 that escaped or died for other reasons and five 
because honeydew had not been administered ad libitum. For A. glycinis, we censored 20 females out of 123 that escaped or died for other reasons and two because honeydew had run out during the trial. All tests performed were analysed using the computer program $\mathrm{R}$ (version 3.3.2 for Macintosh).

\section{RESULTS}

\section{I. Detection and quantification of neonicotinoids in aphid honeydew}

The concentration of clothianidin, the derivate metabolite of thiamethoxam responsible for the insecticidal activity (Nauen et al., 2003; Tomizawa and Casida, 2005), was 9 to 11 times higher in honeydew excreted by aphids feeding on soybean plants whose seeds were coated either with thiamethoxam $(46.76 \pm 27.17 \mathrm{ppb})$ or thiamethoxam and fungicides $(36.98 \pm 8.66 \mathrm{ppb})$ than in honeydew from untreated plants $(3.8 \pm 2.37 \mathrm{ppb})$ (GLMM, $\chi_{2}^{2}=13.57, P=0.001$; Figure 1 and Table S1). These concentrations of clothianidin were similar when they were collected 30-37 or 40-43 days after sowing the soybean (GLMM; days after treatment: $\left.\chi_{1}^{2}=1.18, P=0.27\right)$ with no significant interaction between treatment and days after treatment $\left(\chi_{2}^{2}=4.92, P=0.08\right)$.

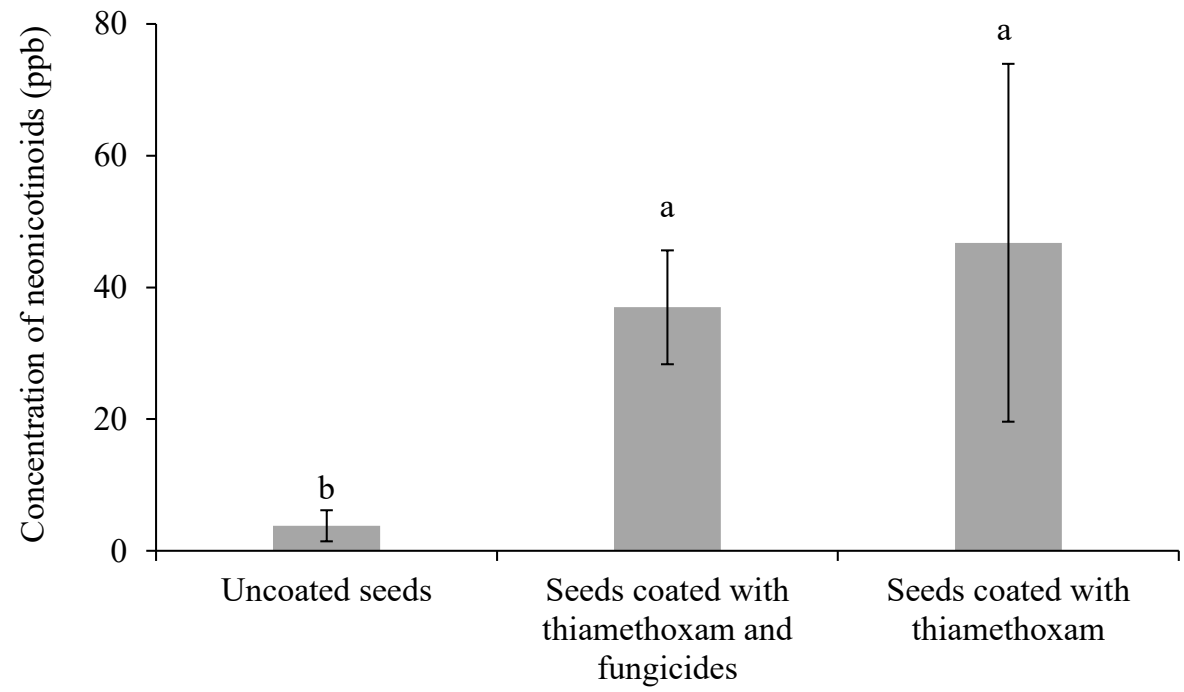

Source of honeydew

Figure 1 | Neonicotinoid concentration in honeydew samples. Concentration (mean $\pm \mathrm{SE}$ ) of clothianidin in honeydew excreted by Aphis glycines feeding on plants whose seeds were uncoated or had been coated either with thiamethoxam only or thiamethoxam and fungicides. Columns with different letters are significantly different from each other (GLMM, based on gamma distribution, $P<$ 0.01 ; number of plots per treatment $=14$ to 16 ). 


\subsection{Longevity of beneficial insects}

The longevity of female predatory midges that fed on honeydew produced by aphids feeding either on plants from seeds coated with thiamethoxam only or thiamethoxam plus fungicides was significantly shorter (median values 7 and 10 days, respectively) than of those fed on honeydew produced by aphids on untreated plants (median: 14 days) $($ Cox's Proportional Hazards $=12.69, P=0.002)($ Fig 2).

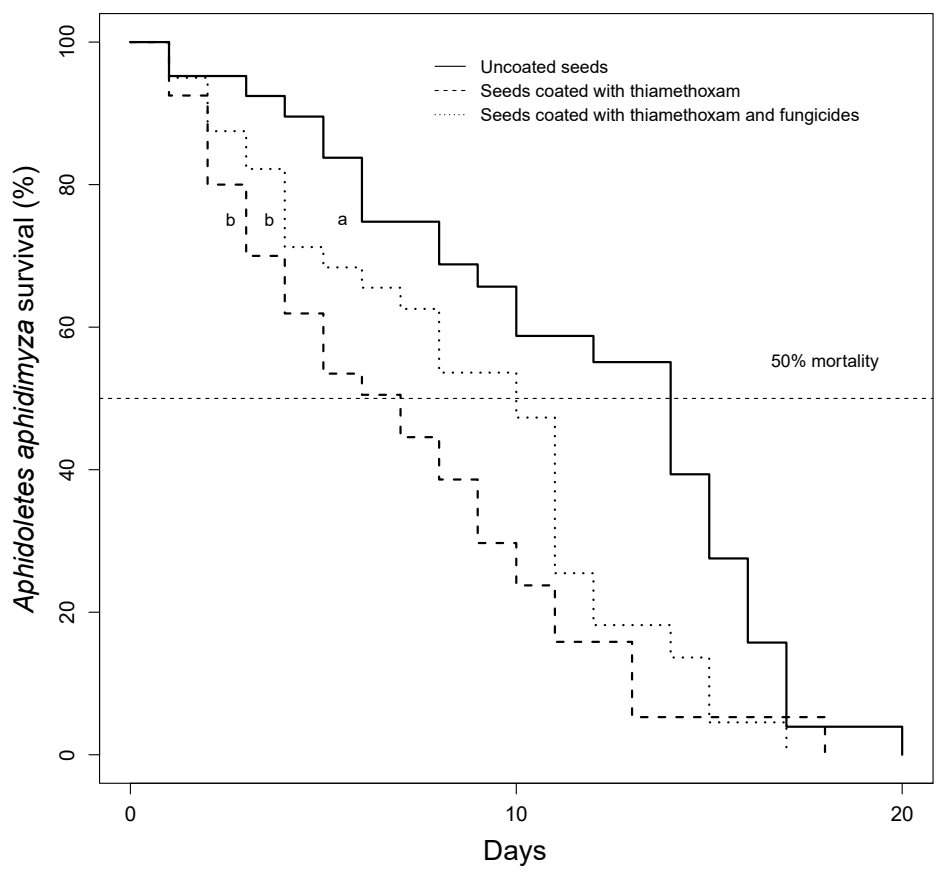

Figure 2 | Survival curves of the predatory midge Aphidoletes aphidimyza. Female midges were fed on honeydew excreted by soybean aphids feeding on plants whose seeds were uncoated or coated either with thiamethoxam only or thiamethoxam and fungicides.

The longevity of female A. certus parasitoids feeding on honeydew produced by aphids feeding on plants whose seeds had been coated with thiamethoxam only or with thiamethoxam plus fungicides was also significantly shorter (median: 12 and 14 days respectively) than that of females fed on honeydew produced by aphids feeding on untreated plants (median: 17 days) (Log-rank Test $=7.3, P=0.03$ ) (Figure 3 ). The longevity of $A$. glycinis females was not statistically different between the three honeydew types (median longevities were 18, 16 and 13 days, respectively for females fed on honeydew excreted by aphids feeding on untreated plants, plants whose seeds were treated with thiamethoxam only or with thiamethoxam and fungicides; Log-rank Test $=3.9, P=0.1$ ) (Figure 4). 


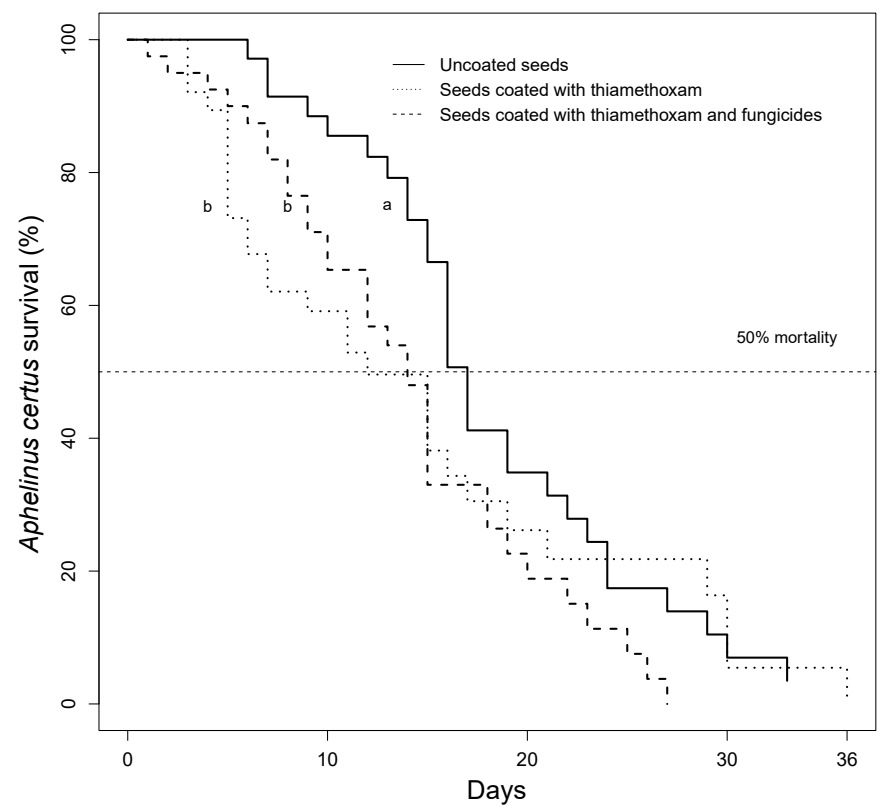

Figure 3 | Survival curves of the parasitic wasp Aphelinus certus. Female wasps were fed on honeydew excreted by the soybean aphids feeding on plants whose seeds were uncoated or coated either with thiamethoxam only or thiamethoxam and fungicides.

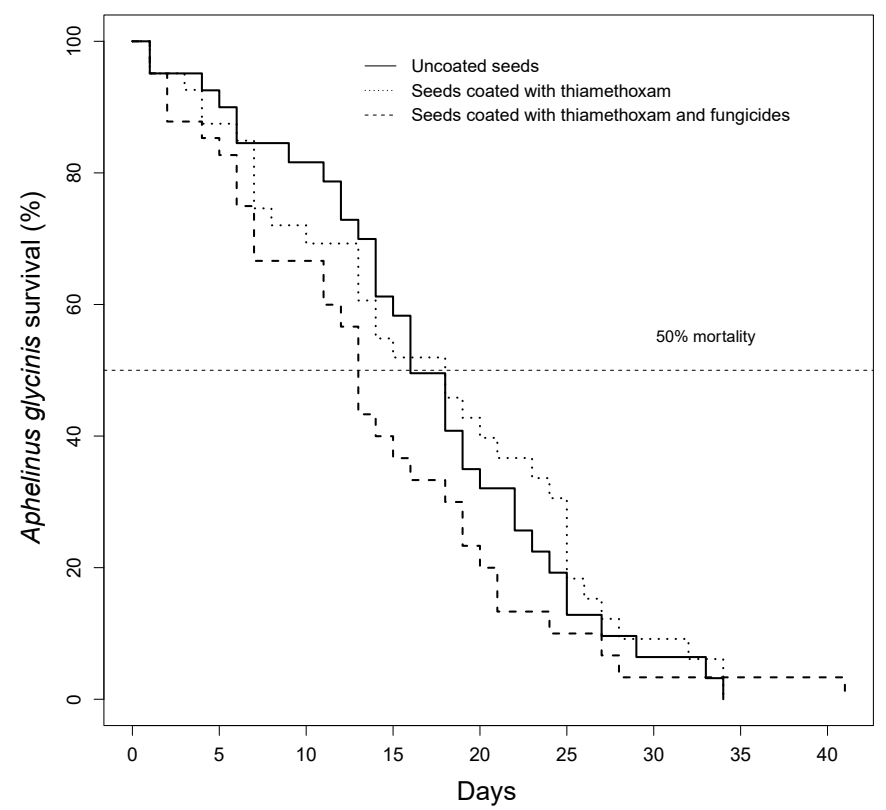

Figure 4 | Survival curves of the parasitic wasp Aphelinus glycinis. Female wasps were fed on honeydew excreted by the soybean aphids feeding on plants whose seeds were uncoated or coated either with thiamethoxam only or thiamethoxam and fungicides. 


\section{DISCUSSION}

Our results demonstrate, for the first time, that neonicotinoids reach honeydew at concentrations that harm biological control agents when plants are grown from soybean seeds coated with neonicotinoids. The soybean aphid excreted honeydew contaminated with $\sim 35-45 \mathrm{ppb}$ of clothianidin when feeding on soybean plants that had been sown 30-43 days previously. We measured the concentration of clothianidin instead of thiamethoxam because the latter is quickly metabolized to clothianidin in plants and insects (Nauen et al., 2003; Tomizawa and Casida, 2005), but some concentration of thiamethoxam might have remained in the plant after 30 days. Therefore, the total concentration of neonicotinoids might be higher than the reported here. In other crops, neonicotinoids derived from coated seeds have been detected in other plant-derived carbohydrate sources such as nectar, extrafloral nectar, or guttation fluids at concentrations as high as 1-8.6 ppb, 1-122 ppb, and 10 ppm, respectively (Girolami et al., 2009; Goulson, 2013; Jones et al., 2020; Rundlöf et al., 2015). However, these carbohydrate sources are absent in soybean agricultural landscapes where honeydew is the main, or only, carbohydrate source for biological control agents (Dieckhoff et al., 2014; Lee et al., 2006) and other beneficial insects.

We also detected low levels of clothianidin in honeydew excreted by aphids feeding on soybean plants from untreated seeds. This result might be explained by two nonexclusive reasons. First, plants might have absorbed residues from previous planting years as it was suggested by Krupke et al. (2017). In our study, soybean and corn seeds coated with neonicotinoids were sowed in some portions of the same field the previous year, 2018. Second, rainwater might have transported neonicotinoids from adjacent plots. Neonicotinoids are water soluble and plants take up only $2-20 \%$ of the neonicotinoid treatment with the remainder leaching into waterways (Sanchez-Bayo, 2014). In our study, it rained in 15 of the 31 days between sowing and honeydew collection (from June 19 until July 19, 2019) (US Climate Data, 2019). These rains caused intermittent runoff and, while we separated plots by 7 meters, neonicotinoids might have moved from treated to untreated plots. However, this second reason seems less plausible because the field was flat and the soil sandy.

Honeydew contaminated with clothianidin from treated seeds reduced the longevity of two of the main biological control agents of the soybean aphid, the predatory midge and the parasitic wasp A. certus, when compared to the honeydew associated with non-treated seeds. Therefore, in this proof-of-concept study, we have demonstrated, for the first time, that neonicotinoids from coated seeds can reach honeydew and harm biological control 
agents. Further research will be necessary to evaluate the effects of honeydew contaminated with neonicotinoids derived from coated seeds on the disruption of biological control under field conditions. This research is likely unfeasible because many parameters of the biological control agents might be affected by neonicotinoids, e.g. immature parasitoids developing in contaminated hosts, contaminated prey for predators, biological control agents searching on plant surfaces contaminated with neonicotinoid dust particles, contaminated nectar from adjacent plants (Goulson, 2013; Krupke et al., 2012). On the other hand, honeydew excreted by aphids feeding on soybean plants from coated seeds did not result toxic to the parasitic wasp A. glycinis but reduced the longevity of $A$. certus. Previous studies have demonstrated that the toxicity of thiamethoxam is species-specific in parasitic wasps, even within the same genus (Cheng et al., 2018).

The three fungicides (fludioxonil, mefenoxam and sedaxane,) used in the seed treatment did not synergize the toxicity of thiamethoxam. Fludioxonil is a phenylpyrrole fungicide used against a broad-spectrum of early-season pathogens that has limited systemic properties (Camargo, 2016). Therefore, it was not expected to contaminate honeydew. Instead, sedaxane and mefenoxam are systemic fungicides from the pyrazoles and phenylamides groups, respectively. Mefenoxam is one of the most commonly used products in soybean targeting Pythium spp., Phytophthora spp. and other plant pathogens of the order Peronosporales (Monkiedje et al., 2007), while sedaxane has a broader spectrum of activity (Zeun et al., 2013). To the best of our knowledge, only one study has evaluated the toxicity of mefenoxam and thiamethoxam on beneficial insects (Camargo, 2016). This study found no adverse effects on worker honey bees mortality and biological control agents when mefenoxam was administered alone via oral and contact exposure. In contrast, when mefenoxam was combined with thiamethoxam, unclear effects on honeybee mortality and no adverse effects for biological control agents were observed (Camargo, 2016).

\section{CONCLUSIONS}

This study demonstrates that the soybean aphid survives the seed-coated treatment and excretes honeydew that contains neonicotinoids derived from coated seeds. As explained in the introduction, soybean is not the only crop, whose seeds are commonly coated with neonicotinoids, and support a diverse group of aphids and other hemipterans that are resistant to neonicotinoids or tolerant to concentrations that degrade over time (De Ribeiro et al., 2018; van Leeuwen et al., 2010). These hemipterans likely also excrete honeydew contaminated with neonicotinoids rendering the honeydew toxic for biological control agents. Therefore, the prophylactic use of seeds coated with neonicotinoids may 
disrupt biological control through contaminated honeydew, increasing both the costs for growers and the environmental impacts of these major crops. Further studies are necessary to corroborate our results in other major crops.

Nowadays, only the European Union (which represents less than 4\% of the global agricultural land) has banned the application of neonicotinoids after a risk assessment report carried out by the European Food Safety Authority. This risk assessment, as well as others carried out by other environmental agencies, did not consider honeydew as route of exposure to beneficial insects. This route is especially relevant because environmental agencies are now evaluating the use of neonicotinoid coated-seeds in crops that are harvested before the flowering period, without considering that these plants can hold phloem-feeding herbivores that excrete contaminated honeydew as we demonstrate here under field conditions. Therefore, honeydew should be included in future environmental risk assessments as a route of exposure to neonicotinoids applied as seed coating.

\section{ACKNOWLEDGEMENTS}

We thank Syngenta Crop Protection for providing the seeds for the experiments. We acknowledge the research assistants Sarah Wood, Sierra Baum, and Amber Johnson who helped enormously with field and laboratory work. This research was partially funded by an INIA project (Project No. RTA2017-00095), an "Europa Excelencia" project (EUR2020-112293), the Conselleria d'Agricultura, Pesca i Alimentació de la Generalitat Valenciana, a grant from the University of Minnesota Rapid Agricultural Response Program, and the Minnesota Agricultural Experiment Station. J.G.C was supported by the Spanish Ministry of Economy and Competitiveness, Ramón y Cajal Program (RYC2013-13834) and M.C-A was recipient of grant from INIA (CPD2016-0085). 


\section{SUPPORTING INFORMATION}

Table S1 | Clothianidin detection in honeydew for each treatment, day and plot.

\begin{tabular}{|c|c|c|c|c|c|c|}
\hline Treatment & $\begin{array}{c}\text { Time } \\
\text { replicate }\end{array}$ & $\begin{array}{c}\text { Days } \\
\text { after } \\
\text { sowing }\end{array}$ & $\begin{array}{c}\text { Plot } \\
\text { number }\end{array}$ & $\begin{array}{l}\text { Neonicotinoid } \\
\text { concentration } \\
(\mathrm{ppb})\end{array}$ & $\begin{array}{c}\text { Honeydew } \\
\text { volume } \\
\left(\mathrm{mm}^{3}\right)\end{array}$ & $\begin{array}{c}\text { Neonicotinoid } \\
\text { concentration } \\
\text { based on } \\
\text { honeydew } \\
\text { volume (ppb) }\end{array}$ \\
\hline \multirow{16}{*}{$\begin{array}{l}\text { Uncoated } \\
\text { seeds }\end{array}$} & \multirow{8}{*}{1} & $30 \& 37$ & 1 & 0 & 0.41 & 0.00 \\
\hline & & $30 \& 37$ & 2 & 0 & 0.66 & 0.00 \\
\hline & & $30 \& 37$ & 3 & 0 & 0.42 & 0.00 \\
\hline & & $30 \& 37$ & 4 & 0.022 & 0.65 & 6.98 \\
\hline & & $30 \& 37$ & 5 & 0 & 0.41 & 0.00 \\
\hline & & $30 \& 37$ & 6 & 0 & 0.12 & 0.00 \\
\hline & & $30 \& 37$ & 7 & 0.0033 & 0.66 & 1.01 \\
\hline & & $30 \& 37$ & 8 & 0 & 0.58 & 0.00 \\
\hline & \multirow{8}{*}{2} & $41 \& 42$ & 1 & 0.13 & 0.68 & 37.65 \\
\hline & & $41 \& 42$ & 2 & 0 & 0.43 & 0.00 \\
\hline & & $41 \& 42$ & 3 & 0 & 0.27 & 0.00 \\
\hline & & $41 \& 42$ & 4 & 0 & 0.27 & 0.00 \\
\hline & & $41 \& 42$ & 5 & 0 & 0.45 & 0.00 \\
\hline & & $41 \& 42$ & 6 & 0.015 & 0.27 & 10.87 \\
\hline & & $41 \& 42$ & 7 & 0.0005 & 0.70 & 0.16 \\
\hline & & $41 \& 42$ & 8 & 0.14 & 1.42 & 20.27 \\
\hline \multirow{14}{*}{$\begin{array}{l}\text { Seeds } \\
\text { coated with } \\
\text { thiamethoxam }\end{array}$} & \multirow{7}{*}{1} & $30 \& 37$ & 1 & 0.27 & 0.47 & 116.73 \\
\hline & & $30 \& 37$ & 2 & 0.002 & 0.23 & 1.57 \\
\hline & & $30 \& 37$ & 3 & 0.02 & 0.70 & 5.29 \\
\hline & & $30 \& 37$ & 4 & 0.24 & 0.20 & 238.54 \\
\hline & & $30 \& 37$ & 6 & 0.20 & 0.66 & 61.73 \\
\hline & & $30 \& 37$ & 7 & 0.06 & 0.15 & 75.95 \\
\hline & & $30 \& 37$ & 8 & 0.07 & 0.33 & 41.20 \\
\hline & \multirow{7}{*}{2} & $41 \& 42$ & 1 & 0.08 & 0.35 & 47.13 \\
\hline & & $41 \& 42$ & 2 & 0 & 0.35 & 0.00 \\
\hline & & $41 \& 42$ & 3 & 0.03 & 0.43 & 13.85 \\
\hline & & $41 \& 42$ & 5 & 0.09 & 0.35 & 53.21 \\
\hline & & $41 \& 42$ & 6 & 0 & 0.40 & 0.00 \\
\hline & & $41 \& 42$ & 7 & 0 & 0.35 & 0.00 \\
\hline & & $41 \& 42$ & 8 & 0 & 0.84 & 0.00 \\
\hline
\end{tabular}


Table S1 | Continuation.

\begin{tabular}{|c|c|c|c|c|c|c|}
\hline Treatment & $\begin{array}{c}\text { Time } \\
\text { replicate }\end{array}$ & $\begin{array}{l}\text { Days } \\
\text { after } \\
\text { sowing }\end{array}$ & $\begin{array}{c}\text { Plot } \\
\text { number }\end{array}$ & $\begin{array}{l}\text { Neonicotinoid } \\
\text { concentration } \\
\text { (ppb) }\end{array}$ & $\begin{array}{l}\text { Honeydew } \\
\text { volume } \\
\left(\mathrm{mm}^{3}\right)\end{array}$ & $\begin{array}{l}\text { Neonicotinoid } \\
\text { concentration } \\
\text { based on } \\
\text { honeydew } \\
\text { volume (ppb) }\end{array}$ \\
\hline \multirow{16}{*}{$\begin{array}{l}\text { Seeds } \\
\text { coated with } \\
\text { thiamethoxam } \\
\text { and three } \\
\text { fungicides }\end{array}$} & \multirow{8}{*}{1} & $30 \& 37$ & 1 & 0.22 & 0.44 & 102.53 \\
\hline & & $30 \& 37$ & 2 & 0.04 & 0.25 & 28.93 \\
\hline & & $30 \& 37$ & 3 & 0.09 & 0.28 & 61.78 \\
\hline & & $30 \& 37$ & 4 & 0.06 & 0.34 & 36.69 \\
\hline & & $30 \& 37$ & 5 & 0.02 & 0.39 & 8.18 \\
\hline & & $30 \& 37$ & 6 & 0.06 & 0.19 & 66.85 \\
\hline & & $30 \& 37$ & 7 & 0.06 & 0.29 & 41.67 \\
\hline & & $30 \& 37$ & 8 & 0.03 & 0.19 & 28.10 \\
\hline & \multirow{8}{*}{2} & $41 \& 42$ & 1 & 0.07 & 0.44 & 31.63 \\
\hline & & $41 \& 42$ & 2 & 0.23 & 0.40 & 115.26 \\
\hline & & $41 \& 42$ & 3 & 0 & 0.45 & 0.00 \\
\hline & & $41 \& 42$ & 4 & 0 & 0.42 & 0.00 \\
\hline & & $41 \& 42$ & 5 & 0.01 & 0.23 & 10.27 \\
\hline & & $41 \& 42$ & 6 & 0.09 & 0.29 & 63.20 \\
\hline & & $41 \& 42$ & 7 & 0.17 & 0.39 & 88.82 \\
\hline & & $41 \& 42$ & 8 & 0.13 & 0.41 & 64.90 \\
\hline
\end{tabular}


Petri dish lid with mesh-covered hole

b

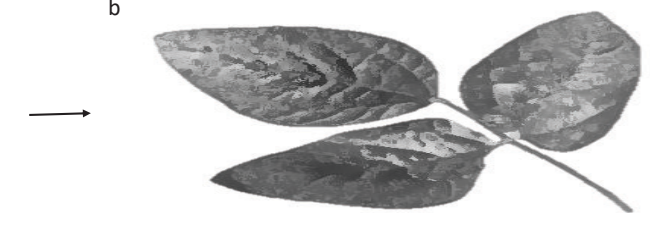

Soybean leaf infested with 50-100 Aphis glycines

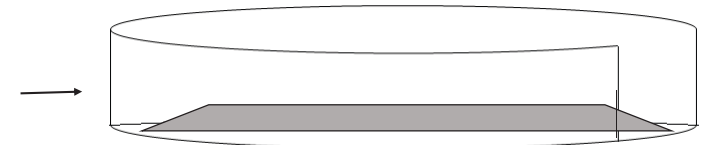

Figure S1 | Honeydew collection in exclusion Petri dish. Parafilm squares of $10 \mathrm{~cm} \times 10 \mathrm{~cm}$ were placed inside 14-cm Petri dishes modified with a mesh-covered hole in the lid and a hole in the lateral wall. One aphid infested soybean leaf was inserted through the lateral hole of the Petri dish. The entire Petri dish was supported in place so the infested leaf was suspended above the Parafilm ${ }^{\circ}$ to collect honeydew. 


\section{Chapter 5}

Beneficial insects do not avoid feeding on honeydew contaminated with neonicotinoids and may even prefer feeding on it
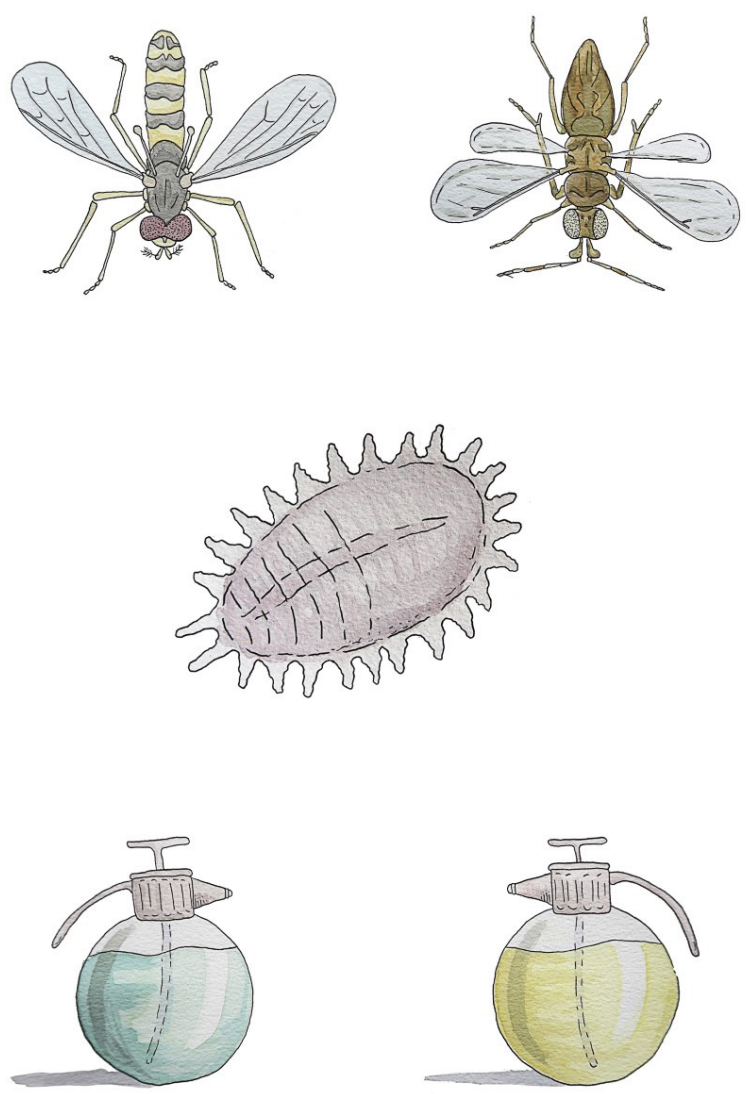



\section{ABSTRACT}

Imidacloprid and thiamethoxam are neonicotinoids toxic for beneficial insects that feed on plant-derived food sources rich in carbohydrates (i.e. nectar, guttation or honeydew) contaminated with these insecticides. Several studies have demonstrated that beneficial insects cannot discriminate between carbohydrate food sources uncontaminated and contaminated with neonicotinoids. However, this has never been tested for honeydew, the main carbohydrate food source in many agricultural landscapes. To address this, we examined whether the parasitic wasp Anagyrus vladimiri and the hoverfly Sphaerophoria rueppellii, with and without feeding experience, discriminate between honeydew excreted by hemipterans feeding on plants treated with imidacloprid or thiamethoxam (contaminated honeydew) and honeydew excreted by hemipterans feeding on untreated plants (uncontaminated honeydew). The hoverfly did not discriminate between honeydew excreted by hemipterans feeding on trees treated with thiamethoxam and on untreated trees, independent of its feeding experience and active ingredient. The parasitic wasp discriminated between honeydew excreted by hemipterans feeding on trees treated with thiamethoxam and honeydew derived from hemipterans feeding on untreated trees, when wasps had no feeding experience or had previously fed on honeydew with thiamethoxam. Contrary to expectations, wasps preferred to feed on honeydew excreted by hemipterans feeding on trees treated with thiamethoxam. These results have profound implications because honeydew is highly abundant and accessible for a plethora of beneficial insects in most agricultural landscapes and they either cannot discriminate or even prefer to feed on honeydew contaminated with thiamethoxam, which is one the most toxic neonicotinoids for beneficial insects. 


\section{INTRODUCTION}

Neonicotinoid insecticides have been extensively used over the last decades because they affect a broad-spectrum of insect pests and appear to have low toxicity to vertebrates (but see the negative effects on birds, mammals, fish, crustaceans, molluscs, annelids and microorganism in Goulson, 2013; Gunstone et al., 2021; Hallmann et al., 2014; Li et al., 2020; Yu et al., 2020). Neonicotinoids move systemically within plants, and hence can be applied using a variety of methods such as foliar sprays, soil drenches, soil granular applications, adding to irrigation systems, injection directly into trees, or by coating the seeds (Jeschke et al., 2011). Once neonicotinoids reach a plant, they are transported systemically to all plant tissues including nectar and pollen, and many beneficial insects i.e. pollinators and biological control agents, that feed on it are harmed (Whitehorn et al., 2012).

Due to their high toxicity for pollinators, the neonicotinoids imidacloprid, thiamethoxam and clothianidin were banned from open field crops in Europe, but this prohibition has been continuously challenged. One important argument against this ban was that some pollinators such as flies or beetles are repelled by neonicotinoid insecticides at field concentrations (Easton and Goulson, 2013). However, several laboratory studies demonstrated later that bumblebees and honeybees do not avoid feeding on food contaminated with neonicotinoids, even at high concentrations that reduce their survival (Arce et al., 2018; Kessler et al., 2015). Even more, these pollinators acquire a preference for neonicotinoid-treated food over time (Arce et al., 2018; Kessler et al., 2015). Probably, the preference for a contaminated food source arises from the pharmacological action of neonicotinoids on nicotinic acetylcholine receptors, by affecting the neural mechanisms required for learning and memory (Kessler et al., 2015; Stanley et al., 2015; Tasman et al., 2021).

It has been recently demonstrated that insecticides can also reach honeydew excreted by hemipterans that feed on plants treated with systemic insecticides, including neonicotinoids (Calvo-Agudo et al., 2021, 2020, 2019; Quesada et al., 2020). This contaminated honeydew can be toxic for several beneficial insect species (Calvo-Agudo et al., 2021, 2020, 2019). Compared to nectar, honeydew is the primary carbohydrate source throughout the year for biological control agents (Hogervorst et al., 2007; Lee et al., 2006; Lundgren, 2009; Luquet et al., 2021; Tena et al., 2016, 2013b; Wäckers et al., 2008), and most pollinators feed on it when nectar is scarce in many agricultural ecosystems (Cameron et al., 2019; Meiners et al., 2017). However, it remains unexplored whether beneficial insects can discriminate between honeydew with or without neonicotinoids. Neonicotinoids 
might affect the chemical composition and infochemicals of honeydew. If these changes occur, beneficial insects might discriminate between contaminated and uncontaminated honeydew. Discrimination between food sources in insects may depend on the feeding experience because some insects have advanced learning abilities (Arce et al., 2018; Loukola et al., 2017). Therefore, feeding experience might affect the ability of beneficial insects to discriminate and avoid honeydew contaminated with neonicotinoids.

Here, we aimed to test whether beneficial insects without feeding experience can discriminate between honeydew excreted by hemipterans feeding on plants treated with the neonicotinoids imidacloprid or thiamethoxam (contaminated honeydew) and honeydew excreted by hemipterans feeding on untreated plants (uncontaminated honeydew). We also investigated whether feeding experience on neonicotinoidcontaining food affects the discrimination between contaminated and uncontaminated honeydew. We expected that beneficial insects would be able to discriminate between uncontaminated and contaminated honeydew, and this discrimination to be dependent on their feeding experience. For these aims, beneficial insects were exposed to choice tests with two types of honeydew and a choice was recorded when the beneficial insect started to feed on one type of honeydew.

\section{System}

We selected citrus as the crop because it is one of the main fruit crops cultivated worldwide being grown on $17.59 \%$ of the global area used for fruit crops (FAOSTAT, 2021). Citrus crops have a diverse and dynamic complex of hemipterans that excrete honeydew during most of the growing season (Pekas et al., 2011; Tena et al., 2013a). Furthermore, neonicotinoids are applied against a diverse range of pest species (GIP Citricos, 2021; Insecticide Resistance Action Committee, 2020; Monzo and Stansly, 2017), and due to their high persistence, neonicotinoids can remain in citrus trees for more than one year (Byrne et al., 2014). The mealybug Planococcus citri (Risso) (Hemiptera: Pseudococcidae) was used because it is a common pest in citrus-producing areas of the world (Urbaneja et al., 2020), whose honeydew increases the longevity and fecundity of beneficial insects (Tena et al., 2013a). As beneficial insects, we selected the parasitic wasp Anagyrus vladimiri (Girault) (Hymenoptera: Encyrtidae) (previously known as A. pseudococci), and the hoverfly Sphaerophoria rueppellii (Wiedemann) (Diptera: Syrphidae). Anagyrus vladimiri is the main biological control agent of P. citri. Sphaerophoria rueppellii was selected because hoverflies represent one of the most important groups of pollinators (Rader et al., 2015), their larvae feed on aphids whereas adults feed on honeydew and nectar, and their populations are declining (Powney et al., 2019). 


\section{MATERIALS AND METHODS}

\section{I. Plant infestation and insecticide application}

The mealybug Planococcus citri was provided by the State Insectary of Generalitat Valenciana (Almassora, Spain), where it was reared on potato sprouts and transported to the Instituto Valenciano de Investigaciones Agrarias (IVIA) (Moncada, Spain) as crawlers (first instar) (Planes et al., 2013).

Forty-five potted clementine trees cv. Clementina de Nules grafted on 'Macrophyla' (Citrus sinensis $\times$ Poncirus trifoliata) were grown in a greenhouse at IVIA until they were one-year-old and $\sim 1 \mathrm{~m}$ high. The environmental conditions in the greenhouse compartments were $22 \pm 5{ }^{\circ} \mathrm{C}, 70 \pm 20 \% \mathrm{RH}$ and the natural photoperiod (FebruaryApril 2019). Clementine trees were watered twice per week and fertilized once per week with Sofertirrig ${ }^{\otimes}$ fertilizer (18-18-18 N-P-K). Plants were infested with $P$. citri crawlers on February 13 and March 12, 2019. To infest them, $1.5 \mathrm{~mL}$ centrifuge tubes half-filled with $P$. citri crawlers were held on the crown of each plant following the methodology of Calvo-Agudo et al. (2019). On April 6, 2019, we applied each insecticide or distilled water (control treatment) directly onto the soil. The neonicotinoids used in this research were thiamethoxam [Thiamethoxam (25\%), Actara 25 WG, Syngenta] and imidacloprid [Imidacloprid (20\%), Confidor 20 LS, Bayer]. A concentration of $0.15 \mathrm{~g}$ of active ingredient of thiamethoxam $/ 0.5 \mathrm{~L}$ of distilled water was applied on 20 different plants. A concentration of $0.375 \mathrm{~mL}$ of imidacloprid/0.5 L in distilled water was applied on 15 different plants. For the controls, $0.5 \mathrm{~L}$ of distilled water was applied on 15 different plants.

\subsection{Honeydew collection}

We collected fresh honeydew from the mealybug P. citri daily from April 8, 2019 ( +2 days after treatment, DAT) to April 10, 2019 (+4 DAT), by placing Parafilm squares of $5 \mathrm{~cm} \mathrm{x}$ $5 \mathrm{~cm}$ below the infested leaf during 24 hours. The collected honeydew for each treatment was labelled and stored at $-20^{\circ} \mathrm{C}$ in Petri dishes until samples were used in the bioassays (Calvo-Agudo et al., 2019; 2020; Hogervorst et al., 2007; Tena et al., 2013b).

\subsection{Beneficial insects}

The parasitic wasp A. vladimiri and the predator-pollinator hoverfly S. rueppellii were obtained as pupae from the commercial companies Koppert Biological Systems S.L (Águilas, Spain) and Biobest Biological Systems (Westerlo, Belgium), respectively. Pupae were introduced into fine-mesh rearing cages $(21 \times 21 \times 21 \mathrm{~cm})$, which were kept in the laboratory at room temperature until adults emerged. Once emerged, both beneficial insects were kept in climatic chambers at $25 \pm 2{ }^{\circ} \mathrm{C}, 75 \pm 10 \% \mathrm{RH}$ and a photoperiod 
of 14:10 h (L:D). For assays of the parasitic wasps, only females were used. Females and males of $S$. rueppellii could not be sexed and, therefore, both sexes were used in the assays.

\subsection{Arena}

The arena consisted of a $140 \mathrm{~mm}$ x $15 \mathrm{~mm}$ glass Petri dish with two Parafilm ${ }^{\bullet}$ pieces containing honeydew of different treatments (Table 1). Parafilm ${ }^{\oplus}$ pieces were placed in opposite extremes of the Petri dish. In order to standardize the amount of honeydew offered in each treatment, Parafilm ${ }^{\odot}$ pieces containing honeydew were observed under the binocular, and cut into pieces of $3.5 \pm 0.5 \mathrm{~cm}^{2}$ with $25 \pm 5$ droplets of honeydew. We selected Parafilm ${ }^{\oplus}$ pieces containing honeydew that had been collected from 7-10 trees per treatment at +3 DAT for the parasitic wasp A. vladimiri and the other 7-10 trees per treatment at +4 DAT for the hoverfly $S$. rueppellii. A piece of wet cotton wool was also placed in the centre of the Petri dish to avoid the crystallization of honeydew, and was removed just before the beginning of the choice test (Tena et al., 2013a). Beneficial insects were subsequently placed in the centre of the Petri dish. A cylinder-shaped filter paper of $10 \mathrm{~cm}$ high was used to enclose the Petri dish, and a cold light was held on top of the Petri dish to illuminate the arena. Petri dishes and Parafilm ${ }^{\circledR}$ pieces with honeydew were renewed for each replicate and individual beneficial insects were tested only once. The position of the pieces of Parafilm ${ }^{\circledR}$ with the honeydew was reversed after every observation. Petri dishes were washed with acetone to avoid contamination from previous replicates. All experiments were conducted inside an airflow cabinet to homogenize the light conditions.

\subsection{Choice tests}

We conducted two-choice tests to investigate whether beneficial insects with different feeding experience can discriminate between honeydew excreted by mealybugs feeding on untreated trees and trees treated with neonicotinoids (imidacloprid or thiamethoxam): i) no feeding experience; ii) feeding experience on honeydew excreted by mealybugs feeding on untreated trees; iii) feeding experience on honeydew excreted by mealybugs feeding on untreated trees (Table 1). A choice was recorded when the beneficial insect started to feed on one of the two types of honeydew. Each replicate lasted until beneficial insects made a choice or 20 minutes. Beneficial insects that did not make a choice within 20 min were recorded as no-choice and excluded from the statistical analyses; these numbers are provided in the figures. All choice tests were carried out until 29-31 beneficial insects responded. Tests were carried out between 10:00 and 15:00 at $22 \pm 2^{\circ} \mathrm{C}$. 


\subsection{No feeding experience}

This experiment was carried out with the parasitic wasp A. vladimiri. Newly-emerged (less than 16 hours old) female wasps were starved for 24 hours in rearing cages $(21 \times 21 \times$ $21 \mathrm{~cm}$ ). Water was provided with wet cotton wool. One day later, wasps were individually placed in glass vials of $3 \mathrm{~cm}$ high and $0.8 \mathrm{~cm}$ diameter covered with wet cotton wool during 30 minutes. Subsequently, females were individually introduced in the arena to carry out two-choice tests between: a) honeydew excreted by mealybugs feeding on untreated trees or trees treated with thiamethoxam; and b) honeydew excreted by mealybugs feeding on untreated trees or trees treated with imidacloprid (Table 1).

This assay could not be carried out for the hoverfly $S$. rueppellii because it only responds to carbohydrate sources after feeding on it (i.e. it needs feeding experience with carbohydrate sources) (Clem et al., 2020 and Supporting information).

Table 1 | Summary of all treatments conducted in the choice test experiment

\begin{tabular}{|c|c|c|}
\hline Beneficial insect & Status before the experiment & Honeydew treatments in choice test \\
\hline \multirow{6}{*}{$\begin{array}{l}\text { Anagyrus } \\
\text { vladimiri }\end{array}$} & \multirow{2}{*}{ Unfed } & Untreated vs thiamethoxam \\
\hline & & Untreated vs imidacloprid \\
\hline & \multirow{2}{*}{$\begin{array}{c}\text { Fed on honeydew excreted } \\
\text { by mealybugs feeding on } \\
\text { untreated trees }\end{array}$} & Untreated vs thiamethoxam \\
\hline & & Untreated vs imidacloprid \\
\hline & $\begin{array}{l}\text { Fed on honeydew excreted by } \\
\text { mealybugs feeding on trees treated } \\
\text { with imidacloprid }\end{array}$ & Untreated vs imidacloprid \\
\hline & $\begin{array}{l}\text { Fed on honeydew excreted by } \\
\text { mealybugs feeding on trees treated } \\
\text { with imidacloprid }\end{array}$ & Untreated vs thiamethoxam \\
\hline \multirow{4}{*}{$\begin{array}{l}\text { Sphaerophoria } \\
\text { rueppellii }\end{array}$} & \multirow{2}{*}{$\begin{array}{c}\text { Fed on honeydew excreted } \\
\text { by mealybugs feeding on } \\
\text { untreated trees }\end{array}$} & Untreated vs thiamethoxam \\
\hline & & Untreated vs imidacloprid \\
\hline & $\begin{array}{l}\text { Fed on honeydew excreted by } \\
\text { mealybugs feeding on trees treated } \\
\text { with imidacloprid }\end{array}$ & Untreated vs imidacloprid \\
\hline & $\begin{array}{l}\text { Fed on honeydew excreted by } \\
\text { mealybugs feeding on trees treated } \\
\text { with imidacloprid }\end{array}$ & Untreated vs thiamethoxam \\
\hline
\end{tabular}


2.5.2. Feeding experience on honeydew excreted by mealybugs feeding on untreated trees

Both beneficial insect species (0-16 hours since emergence) were fed on honeydew excreted by mealybugs feeding on untreated trees during 24 hours in separate rearing cages $(21 \times 21 \times 21 \mathrm{~cm})$. This honeydew was collected at $+2 \mathrm{DAT}$, and was provided ad libitum on several Parafilm ${ }^{\circledast}$ pieces of $25 \mathrm{~cm}^{2}$ during 24 hours and wet cotton wool was introduced in the rearing cage. Afterwards, honeydew was removed and the beneficial insects were starved for 24 hours and subsequently individualized during 30 minutes in glass vials of $3 \mathrm{~cm}$ high and $0.8 \mathrm{~cm}$ diameter (Table 1 ).

2.5.3. Feeding experience on honeydew excreted by mealybugs feeding on trees treated with either thiamethoxam or imidacloprid

Both beneficial insect species (0-16 hours since emergence) were fed on honeydew excreted by mealybugs feeding on trees treated with thiamethoxam or imidacloprid during 24 hours in separate rearing cages $(21 \times 21 \times 21 \mathrm{~cm})$. This honeydew was collected at +2 DAT, and was provided ad libitum on several Parafilm ${ }^{\otimes}$ pieces of $25 \mathrm{~cm}^{2}$ during 24 hours and wet cotton wool was introduced in the rearing cage. Afterwards, honeydew was removed and the beneficial insects were starved for 24 hours and subsequently individualized during 30 minutes in glass vials of $3 \mathrm{~cm}$ high and $0.8 \mathrm{~cm}$ diameter (Table 1).

\subsubsection{Statistical analysis}

The choices for each beneficial insect between honeydew excreted by mealybugs feeding on untreated trees or trees treated with either thiamethoxam or imidacloprid were analysed using a two-sided binomial test. This was done to investigate whether the distribution of beneficial insects differed from 50:50. Beneficial insects that did not make a choice within the $20 \mathrm{~min}$ exposure period were excluded from the statistical analyses. In total, 52 out of 235 (22.6\%) of the parasitic wasps, and 14 out of 134 (10.4\%) of the hoverflies did not make a choice.

\section{RESULTS}

\section{I. Response of A. vladimiri to honeydew excreted by mealybugs feeding on trees treated with thiamethoxam.}

In two-choice tests, $A$. vladimiri parasitic wasps without feeding experience discriminated between honeydew excreted by mealybugs feeding on trees treated with thiamethoxam and on untreated trees. They preferred to feed on honeydew excreted by mealybugs feeding on trees treated with thiamethoxam (binomial test, $P=0.002$ ) (Figure 1a). The same preference was observed when the parasitic wasps had fed on honeydew excreted by mealybugs feeding on trees treated with thiamethoxam (binomial test, $P=0.0005$ ) 
a Honeydew excreted by

Honeydew excreted by

mealybugs feeding on

mealybugs feeding on trees

untreated trees

treated with thiamethoxam

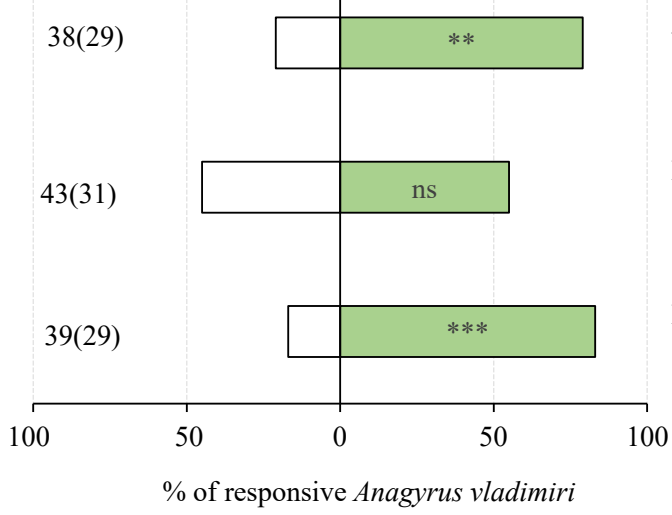

Unfed

Fed on honeydew excreted by mealybugs feeding on untreated trees

Fed on honeydew excreted by mealybugs feeding on thiamethoxam

b Honeydew excreted by Honeydew excreted by mealybugs feeding on mealybugs feeding on trees

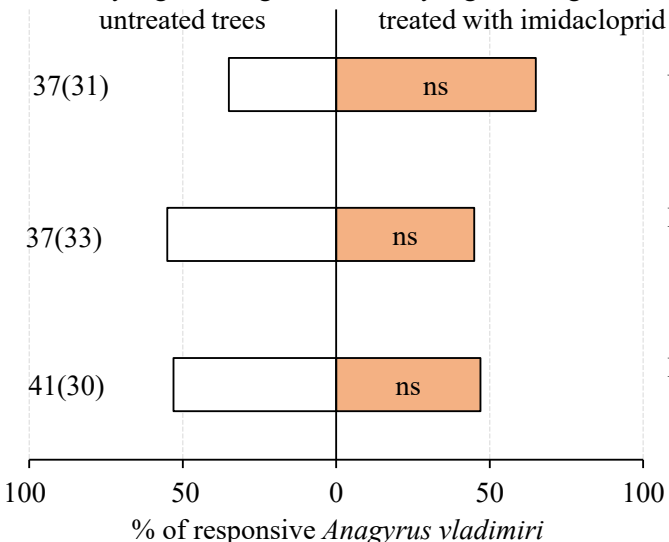

Unfed

Fed on honeydew excreted by mealybugs feeding on untreated trees

Fed on honeydew excreted by mealybugs feeding on imidacloprid

Figure 1 | Behavioural responses of Anagyrus vladimiri parasitic wasps towards honeydew excreted by mealybugs feeding on untreated plants or plants treated with (a) thiamethoxam or (b) imidacloprid. Parasitic wasps had been subjected to three different feeding experiences: unfed, fed on honeydew excreted by mealybugs feeding on untreated trees, or fed on honeydew excreted by mealybugs feeding on trees treated with the corresponding neonicotinoid: thiamethoxam or imidacloprid. Data represent the percentage of parasitic wasps that chose for either of the two honeydew types in choice tests. A selection was annotated when the beneficial insect started to feed on one type of honeydew. The number of tested and responsive (in parentheses) parasitic wasps is given for each test. Asterisks indicate a significant difference within a choice test: ${ }^{* \star} \mathrm{P}<0.001$; ${ }^{\star \star} \mathrm{P}<0.01$; "ns" indicates non-significant (binomial test). 
(Figure 1a). In contrast, A. vladimiri parasitic wasps that had been fed on honeydew excreted by mealybugs feeding on untreated trees did not discriminate between either type of honeydew (binomial test, $P=0.72$ ) (Figure 1a).

\subsection{Response of $A$. vladimiri to honeydew excreted by mealybugs feeding on trees treated with imidacloprid}

In two-choice tests between honeydew excreted by mealybugs feeding on untreated trees and trees treated with imidacloprid, A. vladimiri parasitic wasps did not discriminate between either type of honeydew, independent of their feeding experience (binomial tests: no feeding experience: $P=0.15$; feeding experience on honeydew excreted by mealybugs feeding on untreated trees: $P=0.73$; feeding experience on honeydew excreted by mealybugs feeding on trees treated with imidacloprid: $P=0.85$ ) (Figure $1 \mathrm{~b}$ ).

3.3. Response of S. rueppellii to honeydew excreted by mealybugs feeding on trees treated with thiamethoxam.

In two-choice tests between honeydew excreted by mealybugs feeding on untreated trees and trees treated with thiamethoxam, S. rueppellii hoverflies did not discriminate between either type of honeydew, independent of their feeding experience (binomial tests: feeding experience on honeydew excreted by mealybugs feeding on untreated trees: $P=1$; feeding experience on honeydew excreted by mealybugs feeding on trees treated with thiamethoxam: $P=0.72$ ) (Figure 2a).

\subsection{Response of S. rueppellii to honeydew excreted by mealybugs feeding on trees treated with imidacloprid.}

In two-choice tests between honeydew excreted by mealybugs feeding on untreated trees and trees treated with imidacloprid, S. rueppellii hoverflies did not discriminate between either type of honeydew, independent of their feeding experience (binomial tests, feeding experience on honeydew excreted by mealybugs feeding on untreated trees: $P=0.20$; feeding experience on honeydew excreted by mealybugs feeding on trees treated with imidacloprid: $P=0.061$ ) (Figure 2b).

\section{DISCUSSION}

Our results show, for the first time, that a beneficial insect without previous feeding experience preferred to feed on plant-derived food sources contaminated with the neonicotinoid thiamethoxam. The parasitic wasp A. vladimiri preferred honeydew excreted by mealybugs feeding on trees treated with thiamethoxam over honeydew from mealybugs feeding on untreated trees, when wasps had no previous feeding experience. 
a Honeydew excreted by Honeydew excreted by mealybugs feeding on mealybugs feeding on trees

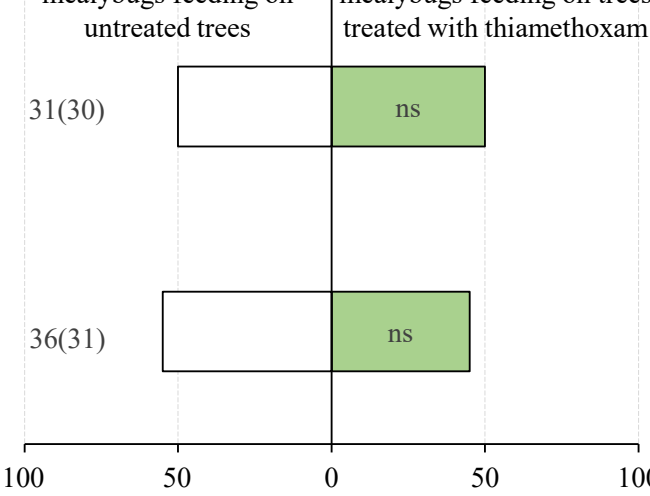

$\%$ of responsive Sphaerophoria rueppellii

b Honeydew excreted by Honeydew excreted by mealybugs feeding on mealybugs feeding on trees \begin{tabular}{l|l} 
untreated trees & treated with imidacloprid
\end{tabular}

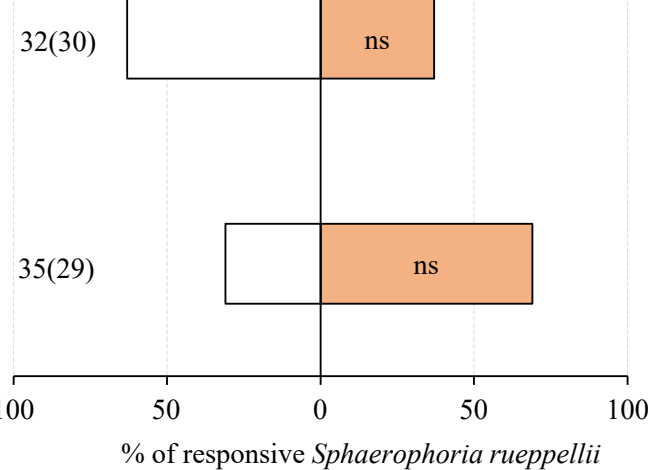

Fed on honeydew excreted by mealybugs feeding on untreated trees

Fed on honeydew excreted by mealybugs feeding on thiamethoxam

Fed on honeydew excreted by mealybugs feeding on untreated trees

Fed on honeydew excreted by mealybugs feeding on imidacloprid

Figure 2 | Behavioural responses of Sphaerophoria rueppellii hoverflies towards honeydew excreted by mealybugs feeding on untreated plants or plants treated with (a) thiamethoxam or (b) imidacloprid. Hoverflies had been subjected to two different feeding experiences: fed on honeydew excreted by mealybugs feeding on untreated trees, or fed on honeydew excreted by mealybugs feeding on trees treated with the corresponding neonicotinoid: thiamethoxam or imidacloprid. Data represent the percentage of hoverflies that chose for either of the two honeydew types in choice tests. A selection was annotated when the beneficial insect started to feed on one type of honeydew. The number of tested and responsive (in parentheses) hoverflies is given for each test. "ns" indicates non-significant (binomial test). 
Moreover, wasps also preferred to feed on honeydew contaminated with thiamethoxam when they had previously fed on it. Bumblebees and honeybees also prefer to feed on sugars contaminated with neonicotinoids once they have fed on it (Arce et al., 2018; Kessler et al., 2015). Further research is necessary to determine why A. vladimiri was able to discriminate and prefer to feed on honeydew contaminated with thiamethoxam. One hypothesis could be that honeydew excreted by mealybugs feeding on treated trees emitted different infochemicals or higher concentrations of infochemicals than uncontaminated honeydew and that this resulted in attraction of the wasps. A recent study has shown that the parasitic wasp Anagyrus dactylopii (Howard) (Hymenoptera: Encyrtidae) is attracted by infochemicals emitted by bacteria present in honeydew excreted by the mealybugs Maconellicoccus hirsutus (Green) (Hemiptera: Pseudococcidae) and Nipaecoccus viridis (Newstead) (Hemiptera: Pseudococcidae) (Fand et al., 2020). If microorganisms are responsible for the results obtained in the present study, the insecticide treatment might have affected them directly or indirectly through the mealybug (i.e. treated mealybug changed the chemical composition of honeydew). Previous studies have shown that neonicotinoids change the bacterial community in the soil (Yu et al., 2020). However, to the best of our knowledge, the effect of neonicotinoids has never been evaluated for the microorganisms that are associated with honeydew and the emission of infochemicals.

Our study also demonstrates that the parasitic wasp A. vladimiri and the predatorpollinator $S$. rueppellii did not discriminate between honeydew excreted by mealybugs feeding on untreated trees and trees treated with the neonicotinoid imidacloprid. In addition, the hoverfly did not discriminate between honeydew excreted by mealybugs feeding on untreated trees and trees treated with thiamethoxam, which is highly toxic for it (Calvo et al., 2019). Therefore, the beneficial insects tested here did not discriminate or, when they did, they preferred to feed on honeydew contaminated with thiamethoxam. In other words, and contrary to our expectations, beneficial insects did not reject to feed on honeydew contaminated with neonicotinoids. These results could have important implications because honeydew-feeding beneficial insects might find honeydew with or without neonicotinoids even within the same plant. In previous studies, we have found honeydew with and without systemic insecticides in samples from the same plant (see Supporting information in Calvo-Agudo et al., 2020). This is likely because the translocation of systemic insecticides can vary among different leaves within the same plant or plant organs (Nauen et al., 2003; Weichel and Nauen, 2004).

Neonicotinoids are highly persistent in plants, water and soil. In fact, they have been detected in fields in which neonicotinoids have not been applied for more than ten years 
(Humann-Guilleminot et al., 2019). Under this scenario, it would be beneficial for insects to discriminate and avoid food sources contaminated with neonicotinoids. However, our results with parasitic wasps and hoverflies and the previous studies with honeybees and bumblebees show that beneficial insects do not avoid feeding on food sources contaminated with neonicotinoids even when they have also access to uncontaminated food sources (Arce et al., 2018; Kessler et al., 2015). These results have profound implications because honeydew and nectar are the main carbohydrate sources in agricultural lands (Lundgren, 2009; Tena et al., 2016; Wäckers et al., 2008). Therefore, these results support the decision of the European Union to ban the neonicotinoids imidacloprid, thiamethoxam and clothianidin for outdoor uses (European Commission, 2020), and to include honeydew as a route of insecticide exposure in environmental risk assessments.

\section{ACKNOWLEDGEMENTS}

We thank Biobest Biological Systems and Koppert Biological Systems for providing the beneficial arthropods used during the bioassays and the State Insectary of Valencia for providing $P$. citri. We acknowledge Natalia Moreno-Ramirez for her valuable help during the experiments. This research was partially funded by an INIA project (Project No. RTA2017-00095) and the Conselleria d'Agricultura, Pesca i Alimentació de la Generalitat Valenciana. M.C-A was recipient of grant from INIA (CPD2016-0085). Authors declare no competing interests. 


\section{SUPPORTING INFORMATION}

\section{I. Effect of feeding status on Sphaerophoria rueppellii}

Previous studies have shown that hoverflies respond to carbohydrate sources only after feeding on them (Clem et al., 2020). For this reason, we tested whether the feeding status affected the response of the hoverfly $S$. rueppellii to honeydew.

To obtain hoverflies without feeding experience, newly emerged hoverflies (0-16 hours since emergence) with only access to water were kept during 24 hours in rearing cages $(21 \times 21 \times 21 \mathrm{~cm})$ with wet cotton wool. Subsequently, unfed hoverflies were individualized during 30 minutes in glass vials of $3 \mathrm{~cm}$ high and $0.8 \mathrm{~cm}$ diameter.

To obtain hoverflies that had fed on honeydew, newly emerged hoverflies (0-16 hours since emergence) with only access to water were fed on honeydew excreted by mealybugs feeding on untreated trees during 24 hours in rearing cages $(21 \times 21 \times 21 \mathrm{~cm})$. This honeydew was provided ad libitum on several Parafilm ${ }^{\circ}$ pieces of $25 \mathrm{~cm}^{2}$ during 24 hours and wet cotton wool was introduced in the rearing cage. Afterwards, honeydew was removed and hoverflies were starved for 24 hours and subsequently individualized during 30 minutes in glass vials of $3 \mathrm{~cm}$ high and $0.8 \mathrm{~cm}$ diameter.

These two groups of hoverflies were tested in two-choice tests following the same methodology explained in the Material and Methods. Here, hoverflies could choose between one piece of Parafilm ${ }^{\circ}$ with honeydew of mealybugs feeding on untreated trees and another piece of Parafilm ${ }^{\circ}$ without food.

Only two of the 15 unfed hoverflies without feeding experience responded but they selected the piece of Parafilm ${ }^{\circledR}$ without honeydew. Therefore, none fed on honeydew. On contrary, 14 out of the 14 hoverflies with feeding experience (fed on honeydew during 24 hours followed by 24 hours of starvation) responded and 12 of these 14 (85.7\%) fed on honeydew. 



\section{Chapter 6}

Insecticide-contaminated honeydew: risks for beneficial insects in agriculture

Miguel Calvo-Agudo, John F. Tooker, Marcel Dicke, Alejandro Tena
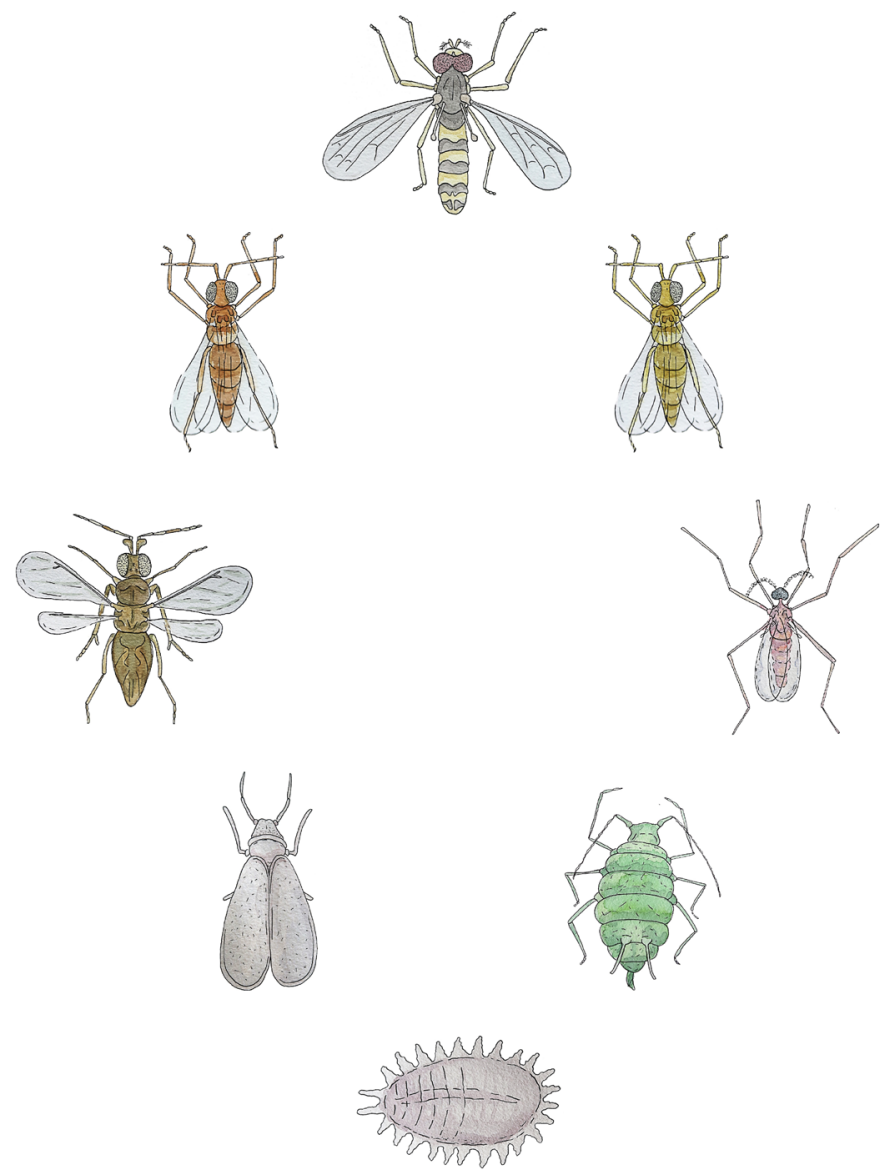



\section{ABSTRACT}

Honeydew is the sugar-rich excretion of phloem-feeding hemipteran insects such as aphids, mealybugs, whiteflies, and psyllids, and can be a main carbohydrate source for beneficial insects in agricultural landscapes. Recent research has revealed that water-soluble, systemic insecticides contaminate honeydew excreted by hemipterans that feed on plants treated with these insecticides. Furthermore, this contaminated honeydew can be toxic to beneficial insects, such as pollinators and biological control agents that feed on it. This route of exposure has now been demonstrated in three crop species, for five systemic insecticides and four hemipteran species, and therefore, we expect this route to be widely available in many agroecosystems. In this perspective paper, we highlight the importance of this route of exposure by exploring: i) potential pathways through which honeydew might be contaminated with insecticides; ii) hemipteran families that are more likely to excrete contaminated honeydew; and iii) systemic insecticides with different modes of action that might contaminate honeydew through the plant. Finally, we analyse several model scenarios in Europe and/or the U.S. where contaminated honeydew could be problematic for beneficial organisms that feed on this ubiquitous carbohydrate source. Overall, this review aims to open a new area of research in the field of ecotoxicology, to shed light on potential undescribed causes of insect declines in agricultural areas, and advocates for this route of exposure to be included in future environmental risk assessments. 


\section{INTRODUCTION}

Honeydew is the sugar excretion product of hemipterans, such as aphids, coccids, whiteflies, and psyllids, that feed on plants. This sugar source is exploited by many beneficial insects including bees, hoverflies, ants, parasitic wasps and predators (Calabuig et al., 2015; Cameron et al., 2019; Hogervorst et al., 2007; Hölldobler et al., 1990; Konrad et al., 2009; Lee et al., 2006; Meiners et al., 2017; Tena et al., 2013b). Compared to other carbohydrate sources present in agricultural lands and some forests, honeydew is highly accessible and can be abundant in nearly all crops and seasons (Lundgren, 2009). Notably, it was recently demonstrated that honeydew can contain insecticides that can negatively influence beneficial insect species (Calvo-Agudo et al., 2021, 2020, 2019). More specifically, it was shown that hemipterans feeding on plants treated with systemic insecticides (i.e., water-soluble insecticides that can move within plant vascular tissue) excreted honeydew laden with the active ingredient of the insecticides or its metabolites, and the honeydew was toxic to insects that consume it (Calvo-Agudo et al., 2021, 2020, 2019; Quesada et al., 2020).

Honeydew as route of exposure to water-soluble insecticides has now been demonstrated for four species of honeydew producers belonging to four different families of hemipterans, five systemic insecticides with four different modes of action and translocation routes, and three plant species (Calvo-Agudo et al., 2020, 2019; Quesada et al., 2020). This route of exposure, therefore, is likely to be abundant in agroecosystems where water-soluble and systemic insecticides are used. The aim of this perspective paper is to discuss the relative importance of this new pathway. For this, we i) review/identify the potential pathways in which honeydew can be contaminated with insecticides. We then ii) discuss which hemipteran families are more likely to excrete contaminated honeydew and; iii) provide a list of systemic insecticides with different modes of action that might contaminate honeydew. Finally, we iv) select several scenarios (model crop species and hemipterans) for which honeydew could be problematic for beneficial organisms. The crop species were selected because they have high economic importance in Europe and/or the United States (U.S.), are commonly treated with systemic insecticides, and honeydew can be the main carbohydrate source for beneficial insects in fields of these crops.

\section{HOW CAN SYSTEMIC INSECTICIDES REACH HONEYDEW?}

Broadly, water-soluble systemic insecticides might reach honeydew through three different pathways (Figure 1): 

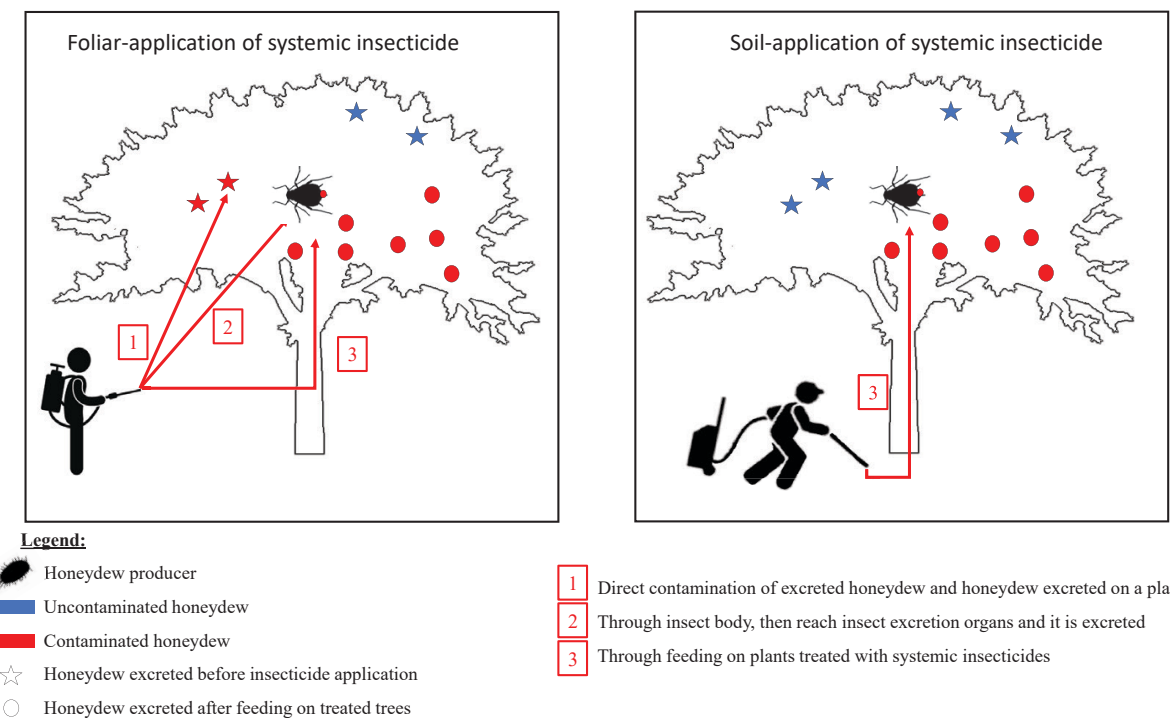

\begin{tabular}{|l|l}
\hline 1 & Direct contamination of excreted honeydew and honeydew excreted on a plant surface \\
\hline 2 & Through insect body, then reach insect excretion organs and it is excreted \\
\hline \hline 3 & Through feeding on plants treated with systemic insecticides
\end{tabular}

Figure 1 | Three pathways in which honeydew can be contaminated with insecticides.

Direct contamination of honeydew. This occurs when honeydew is already present in crops before the insecticide is sprayed or when honeydew is excreted on a plant surface contaminated with insecticides (Figure 1).

Through honeydew producers: insecticides can be directly absorbed into the body of honeydew producers while being sprayed, and honeydew producers might excrete the insecticide via their honeydew (Figure 1).

Through plants and honeydew producers: systemic insecticides are translocated to all parts of the plant, and honeydew producers that feed on treated plants can excrete the insecticide via their honeydew (Calvo-Agudo et al., 2019) (Figure 1). Systemic insecticides are applied using at least four techniques: sprayed, water system, injected in plant (mostly in tree crops) or as seed coats. When systemic insecticides are sprayed, all three pathways of exposure are likely to happen. In contrast, if systemic insecticides are applied in the water system (Calvo-Agudo et al., 2020), injected in the trunks or used as seed coats (Calvo-Agudo et al., 2021), only this third pathway can occur. Within this third pathway, systemic insecticides might reach honeydew under six possible scenarios (Figure 2): 


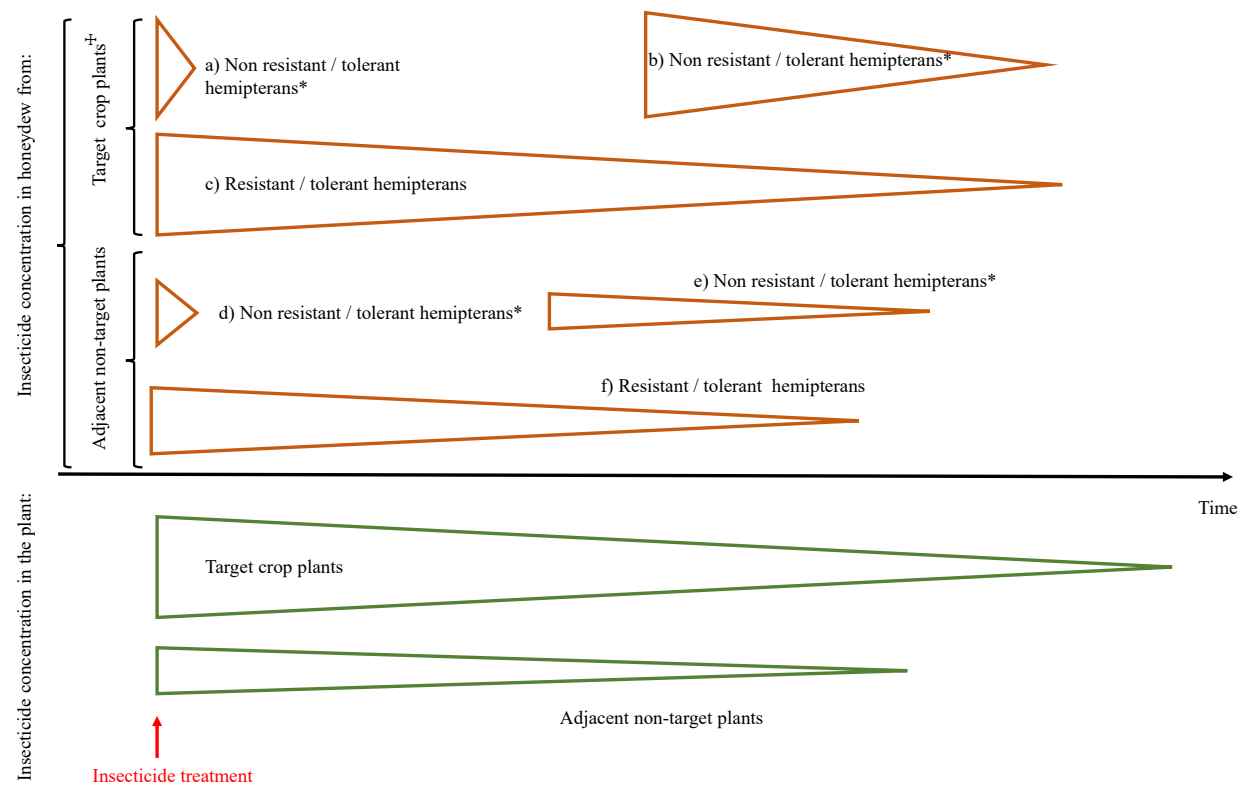

Figure 2 | Five proposed scenarios through which plant-incorporated insecticides can reach honeydew excreted by hemipterans. The width of triangles and rhombus represents insecticide concentration in plants (green) and honeydew (orange) after an insecticide treatment (red arrow). ${ }^{*}$ Resistant / tolerant hemipterans can occur here too.

\section{I. Through hemipterans that feed on treated crop plants}

2.I.I. Non-tolerant/non-resistant hemipterans excrete contaminated honeydew before they die

Non-tolerant/non-resistant honeydew producers are able to excrete contaminated honeydew during short periods of time, before they die as a consequence of the ingested insecticide (Figure 2a). This scenario might occur from a few hours after the insecticide application until hemipterans die due to the treatment. For instance, the mealybug Planococcus citri (Risso) (Hemiptera: Pseudococcidae) and the soft scale Toumeyella pini (King) (Hemiptera: Coccidae) excrete honeydew contaminated with systemic insecticides up to five to eight days after treatment (Supporting information in Calvo-Agudo et al., 2019; Quesada et al., 2020). The period in which these non-tolerant/non-resistant honeydew producers are excreting honeydew is likely to depend on the mode of action of the insecticide, mode of application, plant species, and honeydew producer species (Calvo-Agudo et al., 2020).

2.I.2. Non-tolerant/non-resistant hemipterans excrete contaminated honeydew once insecticide concentration decreases in the plant

Non-tolerant/non-resistant hemipterans might also recolonize insecticide treated plants after insecticide concentration has decreased to levels in which they can survive on the 
host plant (Figure 2b). We expect this scenario to be widely abundant because current agriculture is dominated by extensive monoculture crops whose seeds are commonly coated with systemic insecticides. The seeds of cereals, soybean, cotton, sunflower or rapeseed are coated with neonicotinoids everywhere except in Europe, which represents less than $4 \%$ of the world agricultural landscape (Worldbank, 2020). The protection period of systemic insecticides applied on seeds is limited; therefore, plants become infested with honeydew producers when insecticide concentration decreases and excrete contaminated honeydew (Calvo-Agudo et al., 2021). The same scenario might occur when the insecticides are sprayed or applied in the soil or injected on the trunk and the honeydew producers can tolerate low concentrations. In addition to the decrease in insecticide concentration in the plant with time, this second scenario could arise when systemic insecticides are sprayed, but the target plant receives lower volumes of insecticides due to incorrect insecticide application or unfavorable weather conditions.

2.I.3. Tolerant/resistant hemipterans excrete contaminated honeydew while feeding on treated plants until the insecticide is completely degraded

Another common scenario is when honeydew producers are resistant or tolerant to the active ingredient (Figure 2c). This scenario is likely to occur when the tolerant/resistant honeydew producer is not the target species of the insecticide application or when the target species has developed resistance. As one example of tolerant species, mealybugs are tolerant to the active ingredients flonicamid and pymetrozine (Barbosa et al., 2018; El-Zahi et al., 2016; Nagrare et al., 2016; Rezk et al., 2019) that are selectively used in numerous crops against aphids (Belchim, 2020; Syngenta, 2020). Aphids coexist with mealybugs in citrus, where these active ingredients are applied. The mealybug $P$. citri excretes contaminated honeydew when citrus trees are sprayed with either flonicamid or pymetrozine (Calvo-Agudo et al., 2020). Honeydew contaminated with these insecticides harmed the hoverfly Sphaerophoria rueppellii (Wiedemann) (Diptera: Syrphidae). Excretion of contaminated honeydew by resistant hemipteran species has yet to be demonstrated; nevertheless, this scenario appears to be plausible because key pest species such as, silverfleaf whitefly Bemisia tabaci (Gennadius) (Hemiptera: Aleyrodidae) and green peach aphid Myzus persicae (Sulzer) (Hemiptera: Aphididae), have developed resistance to more than 40 and 70 active ingredients, respectively, some of which can run systemically in plants (van Leeuwen et al., 2010). To our knowledge, at least 24 species of hemipterans that excrete honeydew are tolerant or have the potential to develop resistance to different systemic insecticides (Table S2). It is important to highlight that tolerant/ resistant hemipterans can excrete contaminated honeydew from a few hours after the treatment until these insecticides or their metabolites are completely degraded in the 
plant. Therefore, we expect that tolerant/resistant hemipterans excrete contaminated honeydew for a longer period of time than non-tolerant or non/resistant hemipteran species (Figure 2).

\subsection{Through hemipterans that feed on non-target plants}

Degradation of pesticides involves many abiotic (i.e. environmental conditions, chemical and photochemical reactions) and biotic factors (such as microorganisms, animals or plants) that determine its persistency in the environment (Boxall et al., 2004; Fenner et al., 2013). Systemic insecticides can persist in the environment from days until a few decades (Goulson, 2013; Humann-Guilleminot et al., 2019). For instance, the half-life $\left(\mathrm{DT}_{50}\right)$ in soil, i.e. time taken for the concentration to decrease to half, of clothianidin is 148-6931 days (Rexrode et al., 2003). However, half-lives are not the only important parameter to be considered, because very low concentrations of active ingredients or metabolites may be harmful for non-target organisms (Desneux et al., 2007; van den Brink et al., 2016).

Before systemic insecticides degrade, they can be transported to adjacent crops, cooccurring weeds, field side vegetation, or adjacent habitats or ecosystems by movement in water or insecticide drift (Goulson, 2013; Greatti et al., 2006; Hladik et al., 2014; Krupke et al., 2012; Pearsons et al., 2021; Tsvetkov et al., 2017) (Figure 2d, e, f). During these movements, systemic insecticides can reach non-target plants, even at concentrations exceeding those of the treated crop (Botías et al., 2015). Once insecticides have been absorbed by non-target plants, they can be ingested and excreted by hemipterans, as explained in the previous pathways for target plants (Figure 2a, b, c).

\section{WHAT HEMIPTERAN SPECIES ARE MORE LIKELY TO EXCRETE CONTAMINATED HONEYDEW?}

The feeding behaviour of hemipterans might also affect excretion of contaminated honeydew. For instance, whiteflies feed mostly on plant phloem (Lei et al., 1997); therefore, they will rarely excrete honeydew contaminated with insecticides that move through the xylem (Bromilow et al., 1990). On the other hand, mealybugs, aphids and psyllids feed frequently on both phloem and xylem and they might excrete insecticides that move through both vessels (Cen et al., 2011; Obok et al., 2018; Spiller et al., 1990). Under field conditions, mealybugs and whiteflies excreted the systemic insecticide pymetrozine, which moves through the xylem and phloem, but only mealybugs excreted flonicamid, an insecticide that moves through the xylem (Figure 3; Calvo-Agudo et al. 2020). 


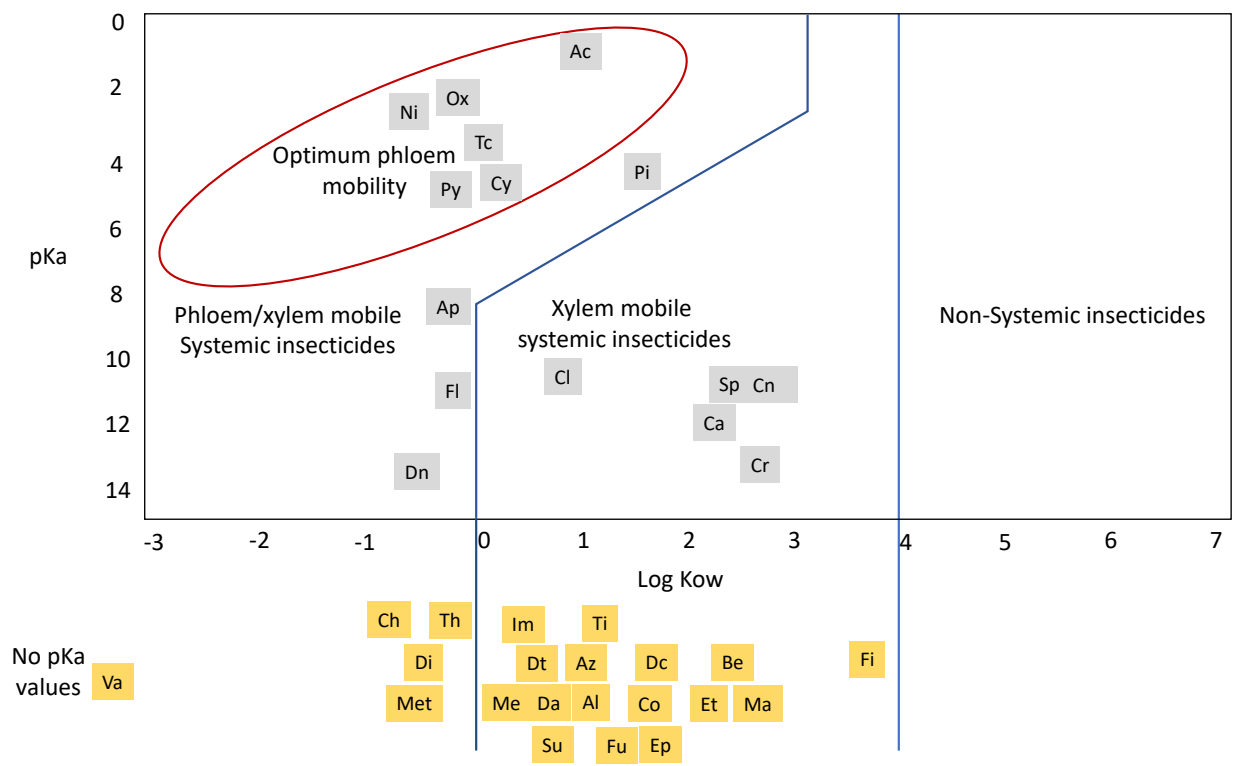

Figure 3 | Systemic behavior of systemic insecticides. Adapted from Bromilow et al. (1990) and University of Hertfordshire (2021). Yellow boxes represent insecticides that have missing $\mathrm{pKa}$ values or that the $\mathrm{pKa}$ cannot be dissociated. $\mathrm{Ac}=$ Acetamiprid, $\mathrm{Al}=$ Aldicarb, $\mathrm{Ap}=$ Acephate, $\mathrm{Az}=$ Azametiphos, $\mathrm{Be}=$ Benzoximate, $\mathrm{Ca}=$ Carbaryl, $\mathrm{Ch}=$ Cartap hydrochloride, $\mathrm{Cl}=$ Clothianidin, $\mathrm{Cn}=$ Chlorantraniliprole, $\mathrm{Co}=$ Carbofuran, $\mathrm{Cr}=$ Chromafenozide, $\mathrm{Cy}=$ Cyromazine, $\mathrm{Da}=$ Dazomet, $\mathrm{Dc}=$ Dichlorvos, $\mathrm{Di}=$ Dicrotophos, $\mathrm{Dn}=$ Dinotefuran, $\mathrm{Dt}=$ Dimethoate, $\mathrm{Ep}=$ Ethiprole, $\mathrm{Et}=$ ethiofencarb, $\mathrm{Fi}=$ Fipronil, $\mathrm{Fl}$ $=$ Flonicamid, $\mathrm{Fu}=$ Flupyradifuron, $\mathrm{Im}=$ Imidacloprid, $\mathrm{Mt}=$ Methamidophos, $\mathrm{Ma}=$ Malathion, $\mathrm{Me}=$ Methomyl, $\mathrm{Ni}=$ Nitenpyran, $\mathrm{Ox}=$ Oxamyl, $\mathrm{Pi}=$ Pirimicarb, $\mathrm{Py}=$ Pymetrozine, $\mathrm{Sp}=$ Spirotetramat, $\mathrm{Su}=$ Sulfoxaflor, $\mathrm{Th}=$ Thiamethoxam, $\mathrm{Ti}=$ Thiacloprid, $\mathrm{Tc}=$ Thiocyclam, $\mathrm{Va}=$ Vamidothion.

\section{WHAT SYSTEMIC INSECTICIDES ARE MORE LIKELY TO CONTAMINATE HONEYDEW?}

Following the previous example, it should be evident that translocation properties of systemic insecticides will also affect honeydew contamination. These properties include the water solubility, the capacity of insecticides to dissolve in lipophilic solutions (nonaqueous), measured as the octanol/water-partition coefficient $\left(\log \mathrm{K}_{\mathrm{ow}}\right)$, and the charge of their molecules at different $\mathrm{pHs}$, measured as the dissociation constant (pKa) (Bromilow et al., 1990). These properties are used to classify insecticides according to their mobility in plants (Figure 3). Here, we summarize some of the main groups of systemic insecticides that are more likely to contaminate honeydew according to: the different pathways explained previously (Figure 2), their mobility in phloem and xylem (Figure 3 ), and their persistence in the environment. These insecticides comprise the following groups: i) neonicotinoids; ii) sulfoximines (mostly the active ingredient sulfoxaflor); iii) flonicamid; iv) pyridine azomethine derivatives (pymetrozine), v) tetronic and tetramic 
acid derivatives (spirotetramat); vi) carbamates; vii) organophosphates. Other groups such as cyromazine, diacylhydrazines (chromafenozide), phenyl-pyrazoles (fipronil), methyl isothiocyanate generators (dazomet), and diamides (chlorantraniliprole, cyclaniliprole and cyantraniliprole) are also likely to contaminate honeydew, but have been excluded from this review because we found little published information on them. Moreover, it is important to highlight that mobility of insecticides in plants can increase with the use of certain copolymers, as occurs with the insecticide fipronil (Bonmatin et al., 2015), that has not been considered here.

\section{I. Neonicotinoids and sulfoximines}

Neonicotinoids and sulfoximines are systemic insecticides that bind to the acetylcholine site on the nicotinic acetylcholine receptors (nAChRs), causing a range of symptoms from hyper-excitation to lethargy and paralysis (Insecticide Resistance Action Committee, 2020). Neonicotinoid insecticides have been extensively used over the last decades because they were considered economic, highly effective against a broadspectrum of insect pests, toxic only for insects (but see that their accumulation has toxic effect on birds: Hallmann et al., 2014; Li et al., 2020), and can be applied in different modes: foliar spray, soil drench, soil granules, injected into irrigation systems, injected directly into trees, or coated on seeds (Jeschke et al., 2011). However, neonicotinoids can be highly persistent in water, plants and soils, where they can remain for years (Table S1) (Byrne et al., 2014; Humann-Guilleminot et al., 2019), and can be highly toxic to beneficial insects, especially pollinators (Pisa et al., 2015). Due to their high persistence and toxicity to beneficial insects, the European Union banned use of the neonicotinoids thiamethoxam, imidacloprid and clothianidin in outdoor crops in 2018. These three insecticides are, however, allowed in most countries outside of Europe. Therefore, we expect neonicotinoids to reach non-target insects when they feed on honeydew that has been contaminated with these three neonicotinoids (Figure 2).

The sulfoximine sulfoxaflor is a systemic insecticide used against hemipterans in a wide variety of crop species (Abdourahime et al., 2019). Sulfoxaflor, like neonicotinoid-based insecticides, is highly soluble in water and can be transported around plant tissues following foliar or seed applications (Siviter et al., 2018). Compared to neonicotinoids, however, it appears to have a relatively short half-life in soil $(\sim 2.2 \mathrm{~d})$ and plant tissues $(\sim 9$ d) (EPA, 2016), diminishing the period in which honeydew can become contaminated (Table S1) (European Food Safety Authority, 2014). Nevertheless, a risk assessment by the European Food Safety Authority (EFSA) indicated high acute oral risks to pollinators (Abdourahime et al., 2019; European Food Safety Authority, 2014); therefore, we expect 
scenarios of honeydew contamination and toxicity similar to neonicotinoids but during shorter periods of time.

\subsection{Flonicamid and pyridine azomethine derivatives}

Flonicamid and pyridine azomethine derivatives such as pymetrozine are systemic insecticides with different modes of action, but ultimately both disrupt feeding and other behaviors in target insects (Belchim, 2020; Syngenta, 2020). Both insecticides can be soil or foliar applied against numerous pests such as whiteflies, aphids, planthoppers or leafhoppers (Belchim, 2020; Syngenta, 2020), but, most mealybug and psyllid species survive exposure to these insecticides (El-Zahi et al., 2016; Qureshi et al., 2014; Rezk et al., 2019). Flonicamid and pymetrozine have high water solubility but their persistence in soil and plants is unclear (Table S1). For instance, flonicamid has under laboratory conditions a soil half-life of $1.1 \mathrm{~d}$ (University of Herthfordshire, 2021), but 2.04-14.2 $d$ in the field (Liu et al., 2014; Wang et al., 2018). In plants, residues of flonicamid or its metabolites can be found in plants 6-21 d after application (Liu et al., 2014; Wang et al., 2018). Tolerant mealybugs and psyllids might, therefore, excrete contaminated honeydew up to $21 \mathrm{~d}$ post-application (Figure $2 \mathrm{~b}, \mathrm{~d}$ ). Compared to neonicotinoids, flonicamid and pymetrozine are less toxic to beneficial insects (Calvo-Agudo et al., 2020, 2019).

\subsection{Tetramic and tetronic acids}

The tetramic-acid derivative spirotetramat inhibits lipid biosynthesis, leading to insect death (Insecticide Resistance Action Committee, 2020). Spirotetramat can be soil or foliar applied against scales, mealybugs, aphids, whiteflies, mites or thrips (Bayer Crop Science, 2020), and has medium mobility in soil, and a very short soil half-life (0.19 d) (Table S1). However, some of its metabolites such as spirotetramat-enol or spirotetramatketohydroxy, exhibit higher mobility and persistence in soil (European Food Safety Authority, 2013b). For instance, the metabolite spirotetramat-ketohydroxy has a half-life of 1.5-14.2 d in soil. Spirotetramat and its metabolites can remain in plants for nearly 30 $\mathrm{d}$ at low concentrations (Chen et al., 2016). When applied, spirotetramat can be excreted through non-resistant/tolerant hemipteran honeydew during short periods of time at high concentrations (Figure 3a). For example, T. pini excretes honeydew contaminated with spirotetramat during at least $8 \mathrm{~d}$ after treatment, before they die from ingesting the insecticide (Figure 3a; Quesada et al., 2020). In addition, tolerant/resistant hemipterans, such as B. tabaci (Bielza et al., 2019), might excrete spirotetramat or its metabolites in their honeydew for long periods of time until the insecticide is degraded (Figure 3b,d). Compared to neonicotinoids, spirotetramat is less toxic for parasitic wasps, predators and 
pollinators (Calvo-Agudo et al., 2019; European Food Safety Authority, 2013b; Planes et al., 2013; Vanaclocha et al., 2013)

\subsection{Carbamates and organophosphates}

Carbamates (CMs) and organophosphates (OPs) contain insecticides that inhibit acetylcholinesterase (AChE), causing hyperexcitation in insects, and some active ingredients within these two groups are systemic (Insecticide Resistance Action Committee, 2020). CMs and OPs are toxic to a broad range of insects (Chowdhury et al., 2012; DiBartolomeis et al., 2019; Donley, 2019; Gupta et al., 2017), and their use has decreased because of their negative effects on invertebrates, birds, fish and mammals (Eddleston et al., 2008). OPs and CMs were routinely applied between 1960 and 2000 and, as a consequence, many hemipterans have developed resistance/tolerance to several active ingredients (Table S2). Most systemic OPs and CMs are highly soluble in water and their persistence in soil and plants varies from low to medium (Table S1). For instance, the CMs pirimicarb and dimethoate can remain in plants for 31 and 38 days, respectively (Szeto et al., 1985). In general, OPs and CMs are highly toxic to many beneficial insects (Mommaerts and Smagghe, 2011). Pirimicarb and dimethoate are particularly likely to contaminate honeydew. Pirimicarb was found in more than $50 \%$ of the samples of surface water (Natale et al., 2018; Struger et al., 2016; Table S1), and many aphids have developed resistance to it (Table S2). Similarly, dimethoate is a common pesticide applied in US fields (van Scoy et al., 2016). More than 816 tons of dimethoate are applied annually mostly in wheat, cotton, corn and alfalfa. A study conducted on the surface water from California detected dimethoate in $9 \%$ of the samples analysed, with a highest concentration of 11.5 ppb (van Scoy et al., 2016). Furthermore, many hemipteran species have developed resistance to it (Table S2). We therefore expect ample risks for beneficial insects to be exposed to these active ingredients when feeding on honeydew from hemipterans on treated plants.

\section{POTENTIAL CROPS IN WHICH HONEYDEW CAN BE CONTAMINATED WITH SYSTEMIC INSECTICIDES.}

In 2018, the global total cropland area was more than 1430 Mhas (FAOSTAT, 2021). In Europe and the U.S., cropland area is about 184 Mhas (12.9\% of the total cropland surface) and 101 Mhas (7.1\%), respectively, and these areas contain several crop species in which honeydew is likely the main carbohydrate source for beneficial insects. We review these scenarios to emphasize the risk posed by insecticide-contaminated honeydew (Figure 4). The examples reviewed here can be extrapolated to other regions, crop species, hemipteran species, and insecticides. 


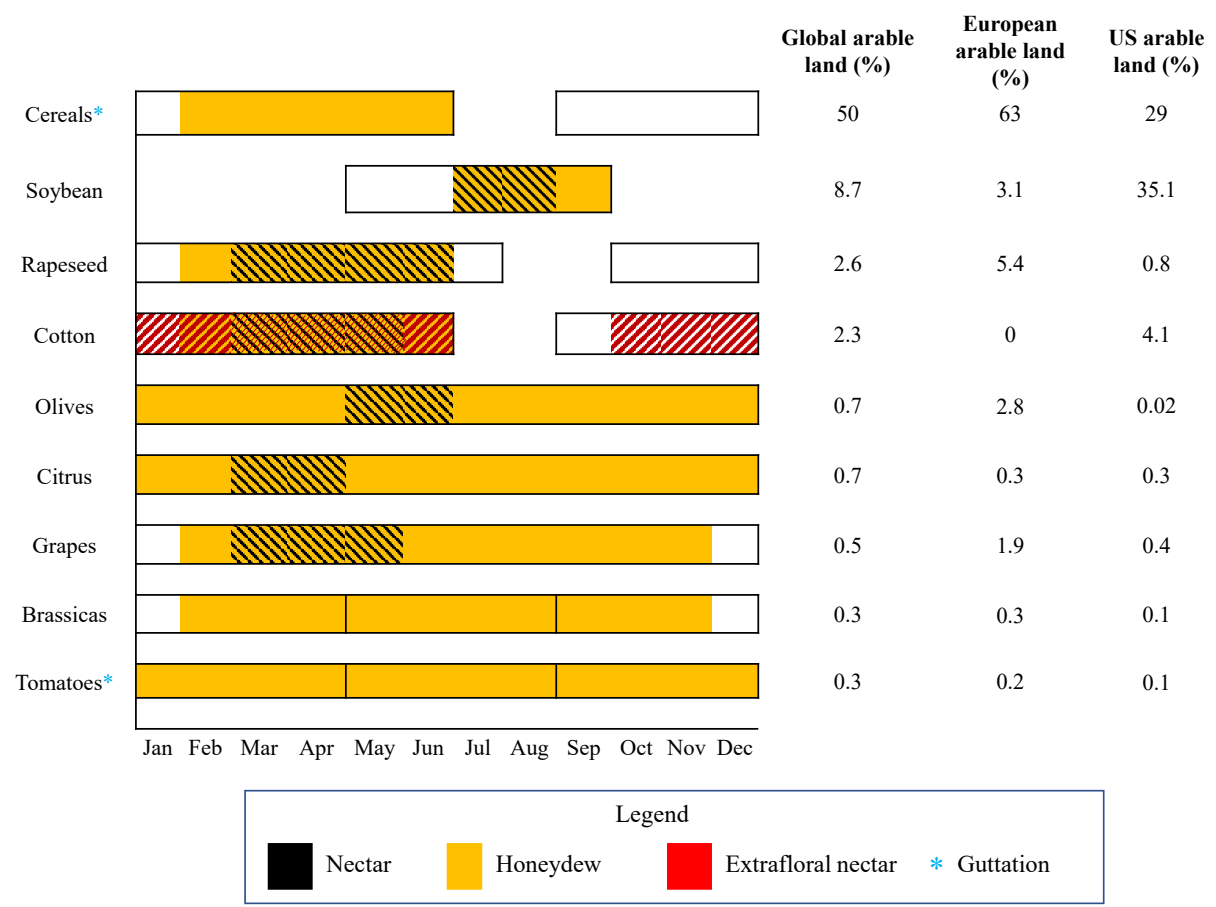

Figure 4 | Potential crops where honeydew contaminated with systemic insecticides might be the most common scenario of exposure to beneficial insects.

\section{I. Extensive crops}

The cereals wheat, maize, rice, barley, rice, sorghum, rye, oat, millet, and triticale occupy nearly $50.5 \%$ of the worldwide harvested area (723 Mhas). In Europe and the U.S., these crops represent 61.7\% (53.9 Mhas) and 29.1\% (53.7 Mhas) of the total agricultural land, respectively (Figure 4). Cereals do not produce nectar, but guttation drops may appear in some humid and windless days (Shawki et al., 2018; Urbaneja-Bernat et al., 2020b). These crops, however, can be infested with many hempiterans that may provide honeydew during the growing season to beneficial insects; these hemipterans include aphid species [E.g. Schizaphis graminum (Rondani), Diuraphis noxia (Kurdjumov), Sitobion avenae (Fabricius), Rophalosiphum maidis (Fitch), R. padi (Linnaeus), A. fabae Scop, M. persicae, Metopolophium dirhodum (Wlk.), root-aphids such as Tetraneura nigriabdominalis (Sasaki) (Hemiptera: Pseudococcidae)] and mealybugs [Brevannia rehi (Lindinger) (Hempitera: Aphididae)]. Therefore, depending on the surrounding landscapes, honeydew might represent a main sugar source for beneficial insects in these agroecosystems. In fact, $59 \%$ of the parasitic wasps and $44 \%$ of the hoverflies collected in spring wheat fields had recently fed on honeydew, and 55\% of the parasitic wasps from winter wheat fields 
had fed on honeydew (Hogervorst et al., 2007). Furthermore, parasitic wasps captured in wheat fields throughout the season can be found to have recently fed on honeydew (Luquet et al., 2021). In Europe, aphids can be treated with systemic insecticides, including acetamiprid, sulfoximines, spirotetramat, and flonicamid. In the U.S., this list includes several organophosphates, carbamates, phenylpyrazoles, and neonicotinoids (Insecticide Resistance Action Committee, 2020). When systemic insecticides are sprayed and aphids are not resistant, they can excrete honeydew during short periods until they die from the insecticide (Figure 2a). However, when seeds are coated with neonicotinoids (Figure 2b), or aphids develop resistance to the insecticide (Figure 2c), the time frame in which they can excrete honeydew with insecticides is likely to be longer.

Seeds of other herbaceous crop species, such as cotton, rapeseed or soybean, are commonly coated with neonicotinoids, such as imidacloprid, thiamethoxam or clothianidin. This is the leading delivery method of neonicotinoids throughout the world (Bonmatin et al., 2015). In fact, in the U.S, more than $50 \%$ of soybeans and $52-77 \%$ of cotton, and $79-100 \%$ of maize hectares were sown with seeds coated with neonicotinoids in 2011 (Douglas and Tooker, 2015) and these amounts appear to continue to increase (Mourtzinis et al., 2019; Tooker et al., 2017). For soybeans grown from seeds coated with thiamethoxam, the soybean aphid Aphis glycines Matsumura (Hemiptera: Aphididae) can colonize plants 25 days after sowing and excrete honeydew containing clothianidin, the derivate metabolite of thiamethoxam, until at least 42 days after sowing (Calvo-Agudo et al., 2021). Honeydew is a common food source for parasitoids in soybean fields (Lee et al., 2006), so it seems likely that honeydew contaminated with neonicotinoids will be problematic for resident natural enemies, consistent with previous research that showed that natural enemies of the soybean aphid were susceptible to neonicotinoid-contaminated honeydew (CalvoAgudo et al., 2021).

Cotton is an example in which three plant-derived sugar sources for beneficial insects may be simultaneously contaminated. Extrafloral nectar is the main food source because it has great nutritional quality and is available throughout the growing season, but it can be tainted with neonicotinoids (Jones et al., 2020). In addition, honeydew excreted by the cotton aphid Aphis gossypii Glover (Hemiptera: Aphididae) is also highly abundant during five months, and can be present at lower quantities during the rest of the season (University of California, 2020). Our research suggests that honeydew from A. gossypii is likely to be contaminated by neonicotinoids coated on seeds, or perhaps other applications later in the season. Floral nectar is the least abundant carbohydrate source because it is available during four to six weeks of the growing period, but it can be contaminated by systemic insecticides 
(Jiang et al., 2018). Contamination of honeydew, however, has been neglected although it is a main food source for beneficial insects in cotton fields (Hagenbucher et al., 2014).

\subsection{Fruit crops}

Citrus, grapes and olives are key crops of southern European and U.S. agriculture. For instance, citrus crops occupy $17.45 \%$ of the global area that is used for fruit crop species of the worldwide harvested area (9.67 Mhas). In Europe and the U.S., this crop represent 0.52 Mhas and 0.28 Mhas, respectively (FAOSTAT, 2021). The brief flowering period only lasts for two/three months (Figure 4), and permanent ground covers that provide additional nectar are scarce (Gómez et al., 2018; Tena et al., 2013b). In contrast, a diverse and dynamic complex of hemipterans feed on citrus and can excrete large amounts of honeydew throughout the year (Pekas et al., 2011; Tena et al., 2013a). In Mediterranean citriculture, there are numerous naturally controlled hemipterans that are often considered secondary pests and rarely need to be controlled chemically (Urbaneja et al., 2020). For instance, aphids [A. spiraecola Patch and A. gossypii (Hemiptera: Aphididae)] are highly abundant early in spring, coccids [Coccus hesperidum L. and Saissetia oleae Olivier (Hemiptera: Coccidae)] and pseudococcids [P. citri] are dominant at the end of the spring and during summer, and whiteflies [Aleurothrixus floccosus (Maskell) (Hemiptera: Aleyrodidae)] can be present on tender leaves in autumn (Pekas et al., 2011). Hence, honeydew is a commonly available food source for beneficial insects, including parasitic wasps of non-honeydew-producing herbivores in these agroecosystems (Calabuig et al., 2015; Tena et al., 2013b). Aphids or whiteflies can be treated with systemic insecticides such as sulfoxaflor, spirotetramat, acetamiprid or flonicamid when they exceed the economic injury level (GIP Citricos, 2021, Insecticide Resistance Action Committee, 2020). While aphids or whiteflies can excrete these insecticides via honeydew during short periods of time (Figure 2a) (unless they develop resistance, Figure 2c), tolerant hemipterans such as $P$. citri can excrete contaminated honeydew for longer periods of time (Figure 2c) (Calvo-Agudo et al., 2020).

In the citrus industry in Florida (U.S.), numerous broad-spectrum systemic insecticides such as organophosphates, carbamates, neonicotinoids, sulfoximines or spirotetramat are applied to control the Asian citrus psyllid, Diaphorina citri Kuwayama (Hemiptera: Liviidae) (Qureshi et al., 2014). This psyllid, which excretes honeydew (Ammar et al., 2013), is a vector of the "Candidatus Liberibacter" pathogen that is responsible for causing 'huanglongbing' (HLB) disease. Diaphorina citri colonizes citrus trees during the flushing periods of spring, summer and fall (Qureshi et al., 2014). Citrus growers tend to apply insecticides ca. twelve times per year against D. citri (Monzo and Stansly, 2017), mostly of systemic insecticides (Insecticide Resistance Action Committee, 2020). 
Some of these insecticides, such as neonicotinoids, can remain in citrus trees for more than one year (Byrne et al., 2014) and hence, while feeding on treated plants, numerous hemipterans can excrete honeydew that contains one or several systemic insecticides.

\subsection{Horticultural crops}

Brassicas such as cauliflower, broccoli, cabbage or kale represent 3.8 Mhas worldwide. In Europe and the U.S., these crops are grown in 278,234 has and 86,194 has, respectively (FAOSTAT, 2021). These crops are harvested before they flower; therefore, depending on the surrounding landscape, beneficial insects active in these crops may encounter the honeydew excreted by aphids or whiteflies during the whole cropping period. In fact, $80 \%$ of Cotesia glomerata (L.) (Hymenoptera: Braconidae) and 55\% of Microplitis mediator (Haliday) (Hymenoptera: Braconidae) parasitic wasps collected in cabbage fields had recently fed on honeydew, whereas only $16 \%$ of the C. glomerata collected in cabbage fields with flowering borders had exclusively fed on nectar (Wäckers and Steppuhn 2003). Planting seeds coated with neonicotinoids in brassica crops has been discussed in Europe because brassicas are harvested before the flowering period and therefore, these crops do not pose any risk to pollinators via nectar (European Food Safety Authority, 2013a). However, once they are tolerant to insecticide concentrations aphids and whiteflies can colonize seed-coated Brassica plants at any plant growth stage and are likely to produce insecticide-contaminated honeydew. The same situation might occur with fipronil. This phenyl-pyrazole was initially not considered a systemic insecticide, but some uptake by plants occurs (European Food Safety Authority, 2013c), especially if commercial formulations contain additional substances that alter the systemic properties (Bonmatin et al., 2015; Dieckmann et al., 2010b, 2010c, 2010a). It has been recently demonstrated that fipronil has sublethal effects on herbivorous insects that feed on brassicas grown from coated seeds (Gols et al., 2020). Therefore, it might be also excreted by hemipterans. Fipronil is currently not allowed to be used in Europe. In the U.S., however, fipronil is allowed for use on potatoes, although its applications are limited because of the potential environmental hazards (Knodel and Shrestha, 2018; Tingle et al., 2003). Horticultural crops such as tomatoes, cucumbers, eggplants, zucchini, etc. are important crops in the U.S. and Europe. For example, tomatoes are planted on 0.49 Mhas worldwide. In Europe and U.S., this crop is planted on 239,724 has and 130,270 has, respectively (FAOSTAT, 2021). Tomato flowers do not contain nectar and thus, honeydew might be an important carbohydrate source for beneficial insects foraging in tomato fields (Figure 5). In Europe, one of the most common systemic insecticides is chlorantraniliprole, which is used against the key pest Tuta absoluta (Meyrick) (Lepidoptera: Gelechiidae) (Biondi et al., 2018). Other systemic insecticides such as spirotetramat, sulfoxaflor, flonicamid or acetamiprid are used against whiteflies [B. tabaci 
and Trialeurodes vaporariorum (Westwood) (Hemiptera: Aleyrodidae)] and/or aphids [A. gossypii or Macrosiphum euphorbiae (Thomas) (Hemiptera: Aphididae)] (Castañé et al., 2020) (Insecticide Resistance Action Committee, 2020). In addition, the neonicotinoids imidacloprid, clothianidin and thiamethoxam can be used under greenhouse conditions (European Commission, 2018) against the above mentioned pests. In the U.S., similar insecticides are allowed as are pymetrozine, organophosphates or carbamates (Donley, 2019). As a result, we expect hemipterans in tomatoes to excrete honeydew contaminated with neonicotinoids, sulfoximines, spirotetramat, flonicamid, pymetrozine, CMs, or OPs for at least short periods of time (Figure 2a, d). In addition, whiteflies and aphids, which are tolerant to chlorantraniliprole (Barrania and Abou-Taleb, 2014), might excrete honeydew with chlorantraniliprole until residues degrade in the plant (Figure 2c,e).

\section{CONCLUSION}

This article shows how beneficial insects such as pollinators and biological control agents can be exposed to contaminated honeydew. Other plant-derived food sources such as nectar, extrafloral nectar or guttation are important routes of insecticide exposure, but their availability is restricted to brief flowering periods (i.e. nectar), a few crop plant species (i.e., those with extrafloral nectaries), or specific climatic conditions (i.e., guttation). In contrast, honeydew can be available during most of the growing season and for many crop species. Here, we first presented several pathways through which systemic insecticides might reach honeydew. Among hemipteran families, mealybugs, aphids and psyllids may excrete honeydew contaminated with systemic insecticides more often than whiteflies because the former families feed on both phloem and xylem. Among the insecticide groups, we suggest that neonicotinoids are the most likely to reach honeydew and negatively affect beneficial insects due to their high persistence in soil, water and plants, their high water solubility and high toxicity. Other insecticides that have lower persistence or toxicity, such as flonicamid or spirotetramat, are less likely to affect beneficial insects via honeydew. We then highlighted some valuable crop species for Europe and U.S. that are commonly infested with hemipterans and treated with different active ingredients, but it must be noted that many other crop species in other parts of the world may be infested with hemipteran species. In this sense, this perspective paper generates many questions in the field of ecotoxicology that will need to be answered in the coming years about the scope of the risk and the ubiquity of exposure to beneficial insect species. Another important aim of this perspective paper is to broadcast this route of exposure to environmental protection agencies and integrated pest management programs that include use of systemic insecticides. 


\section{SUPPORTING INFORMATION}

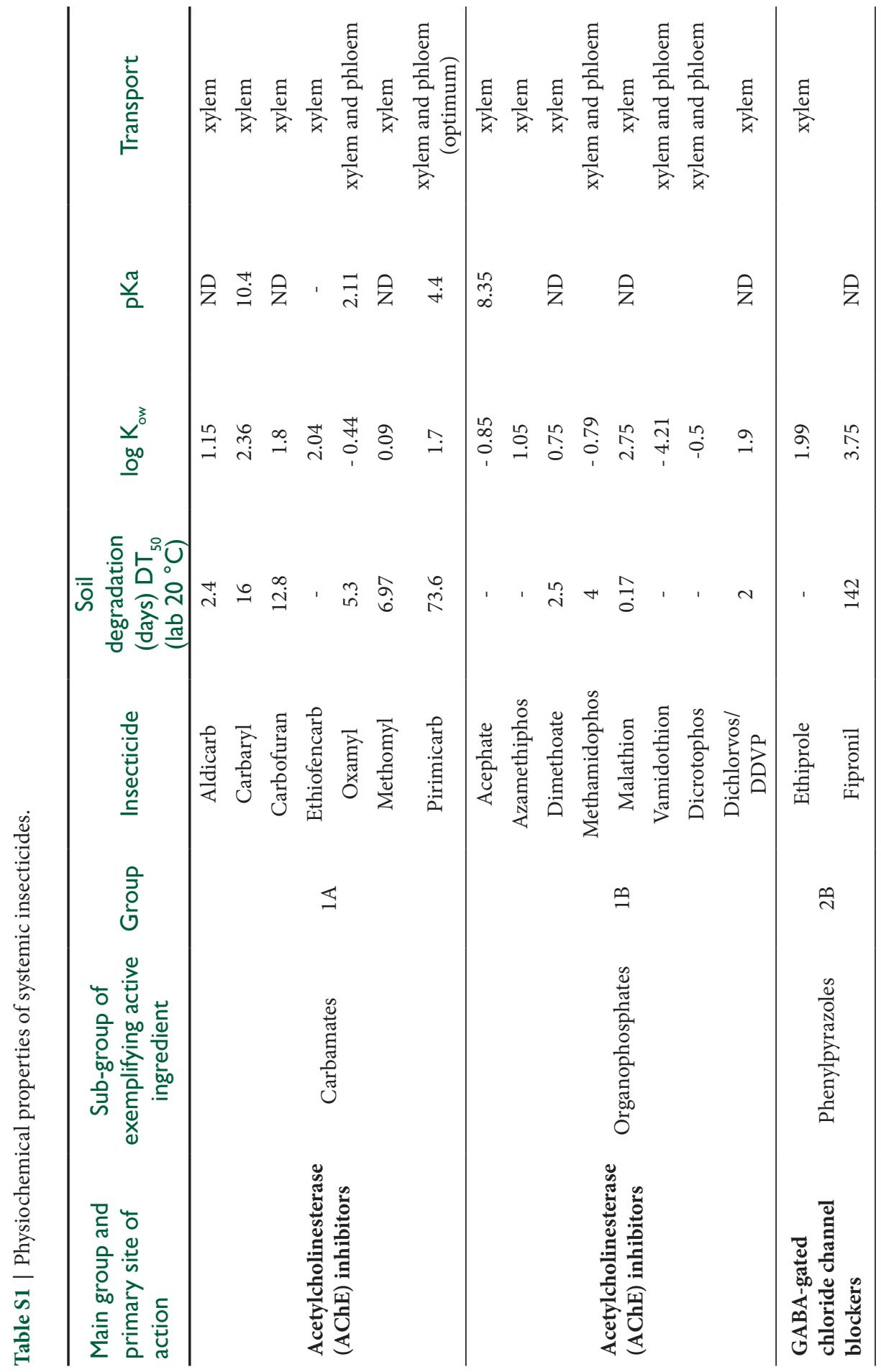




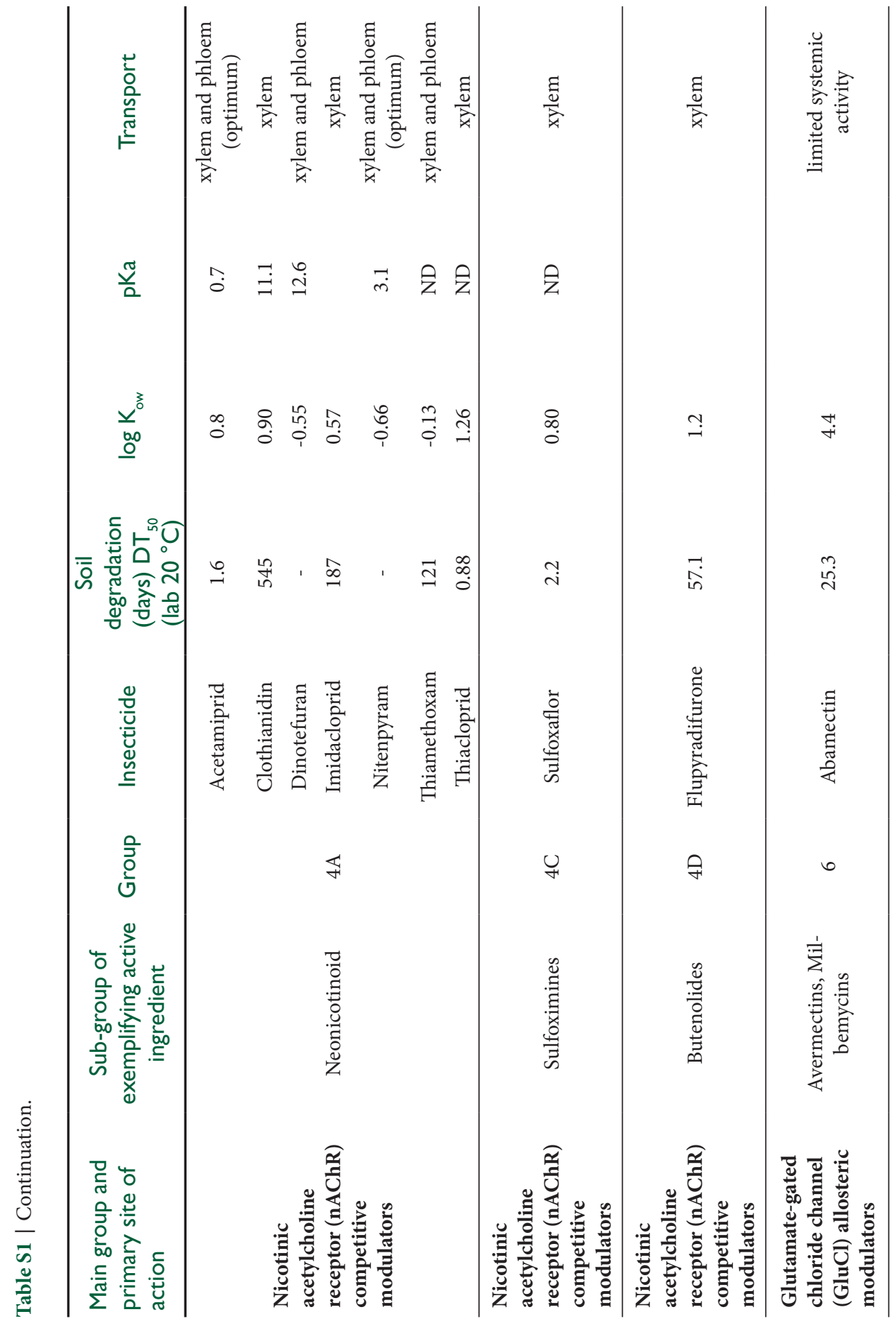




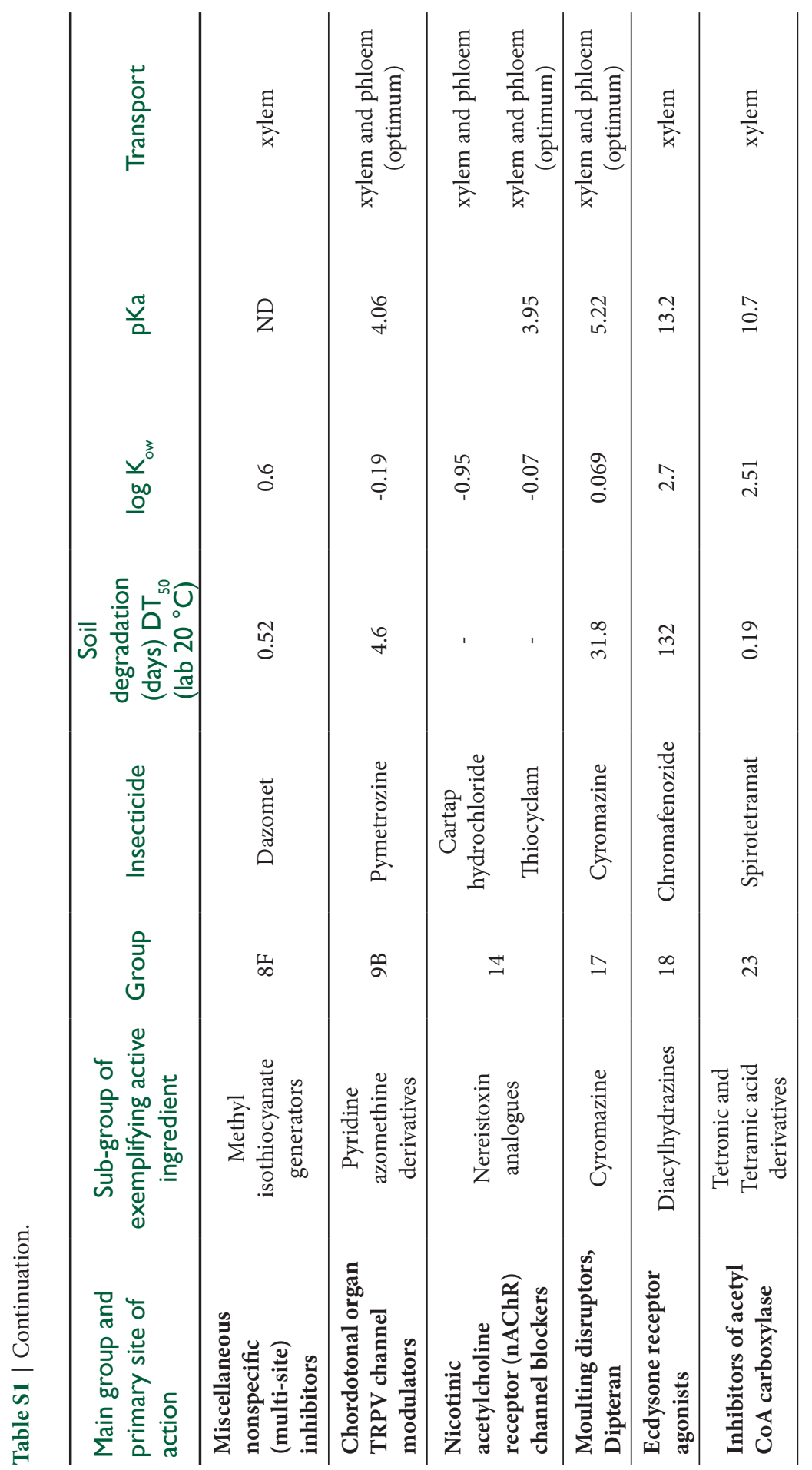




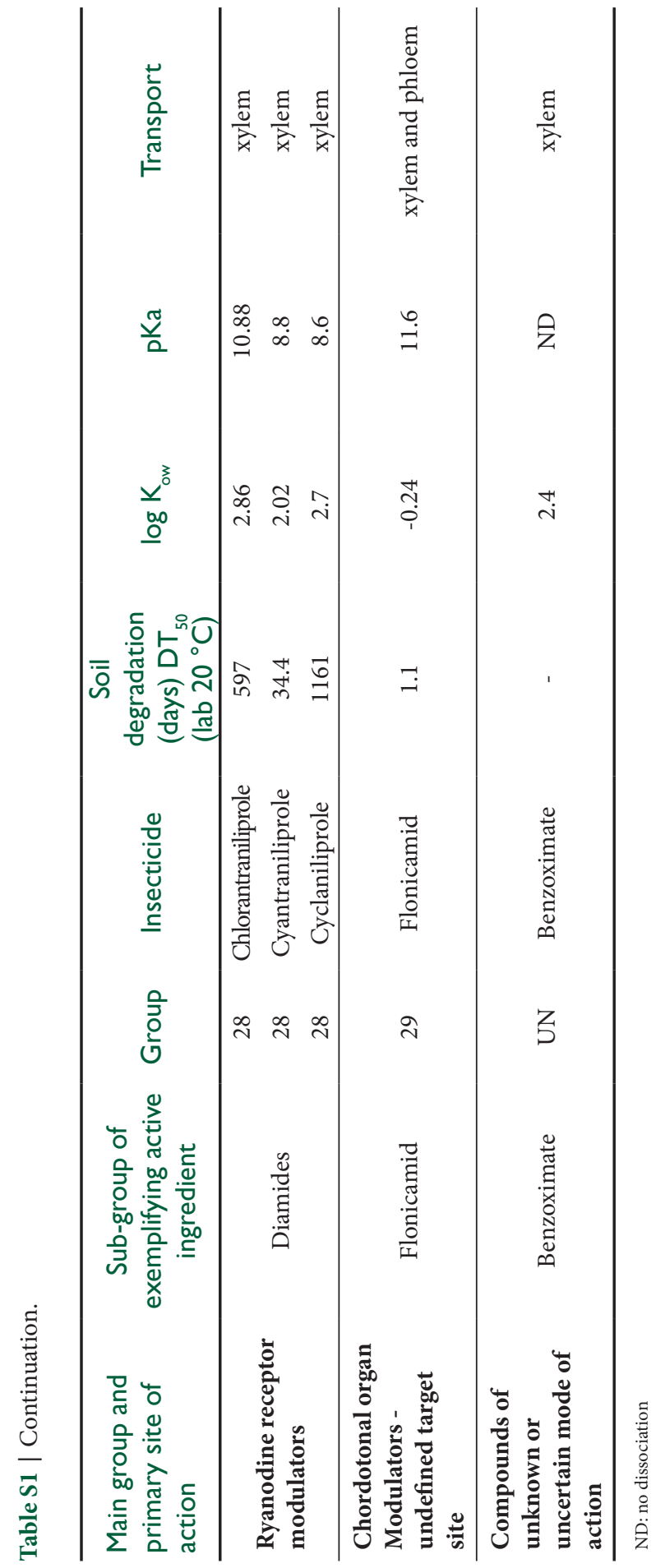




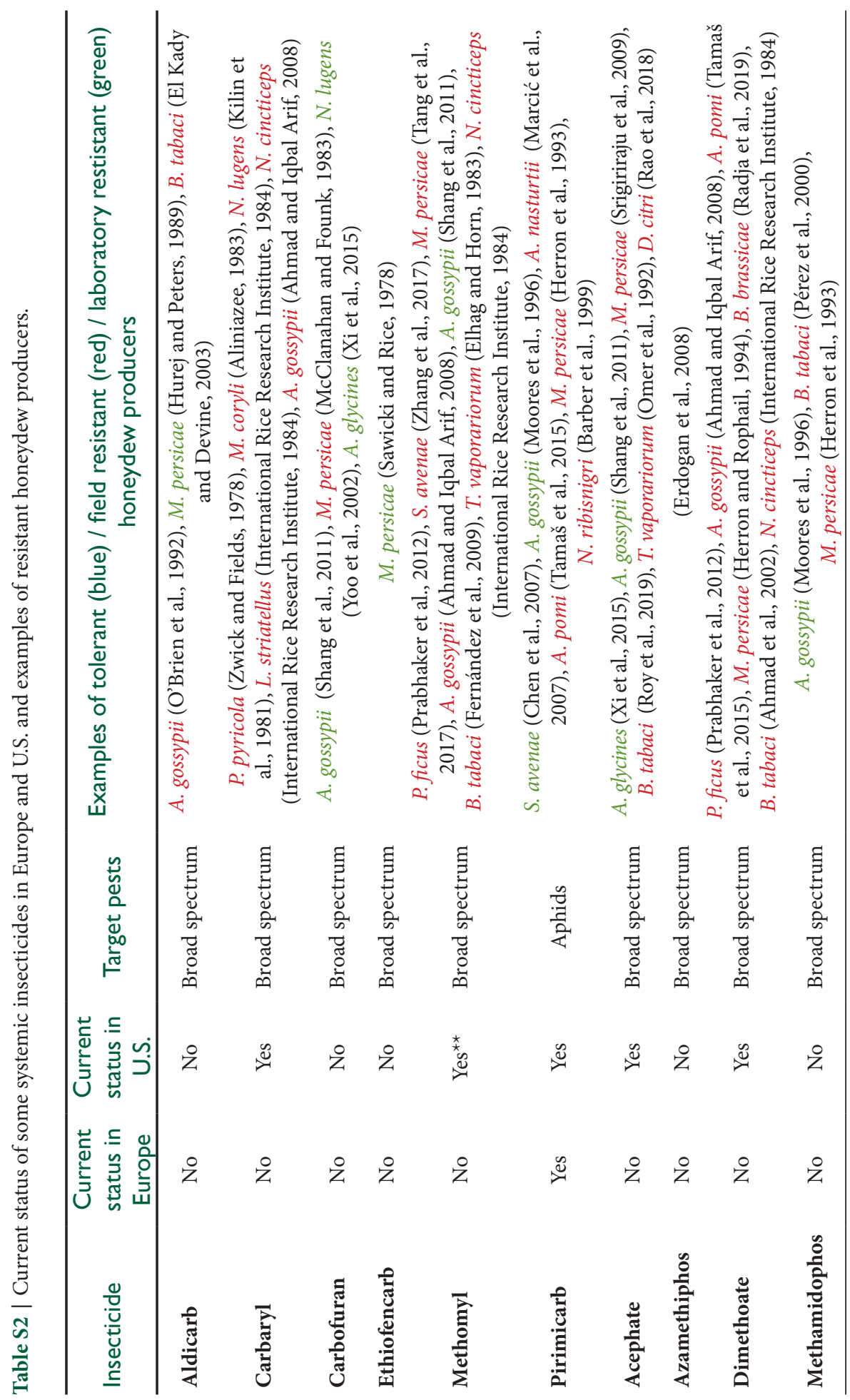




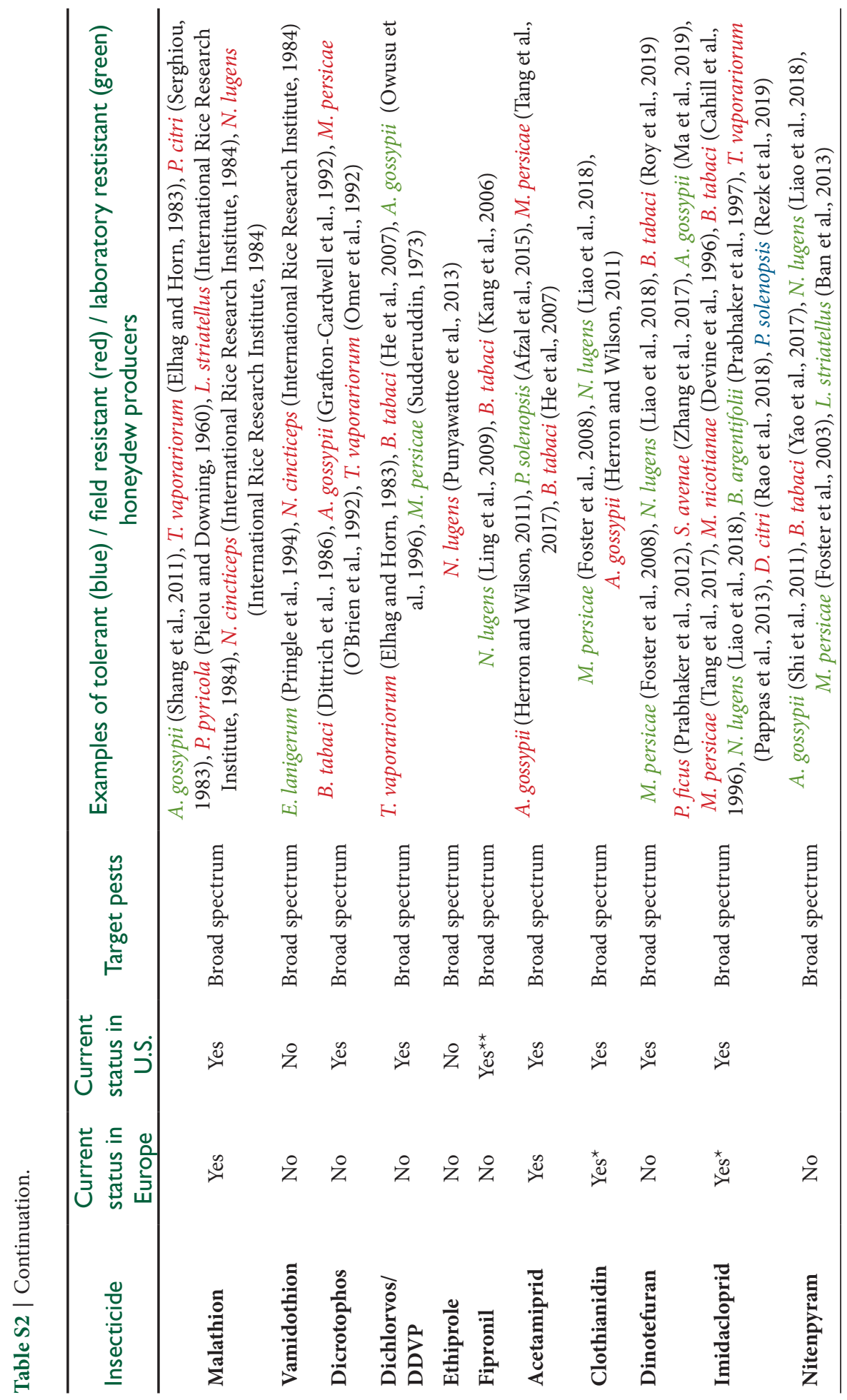




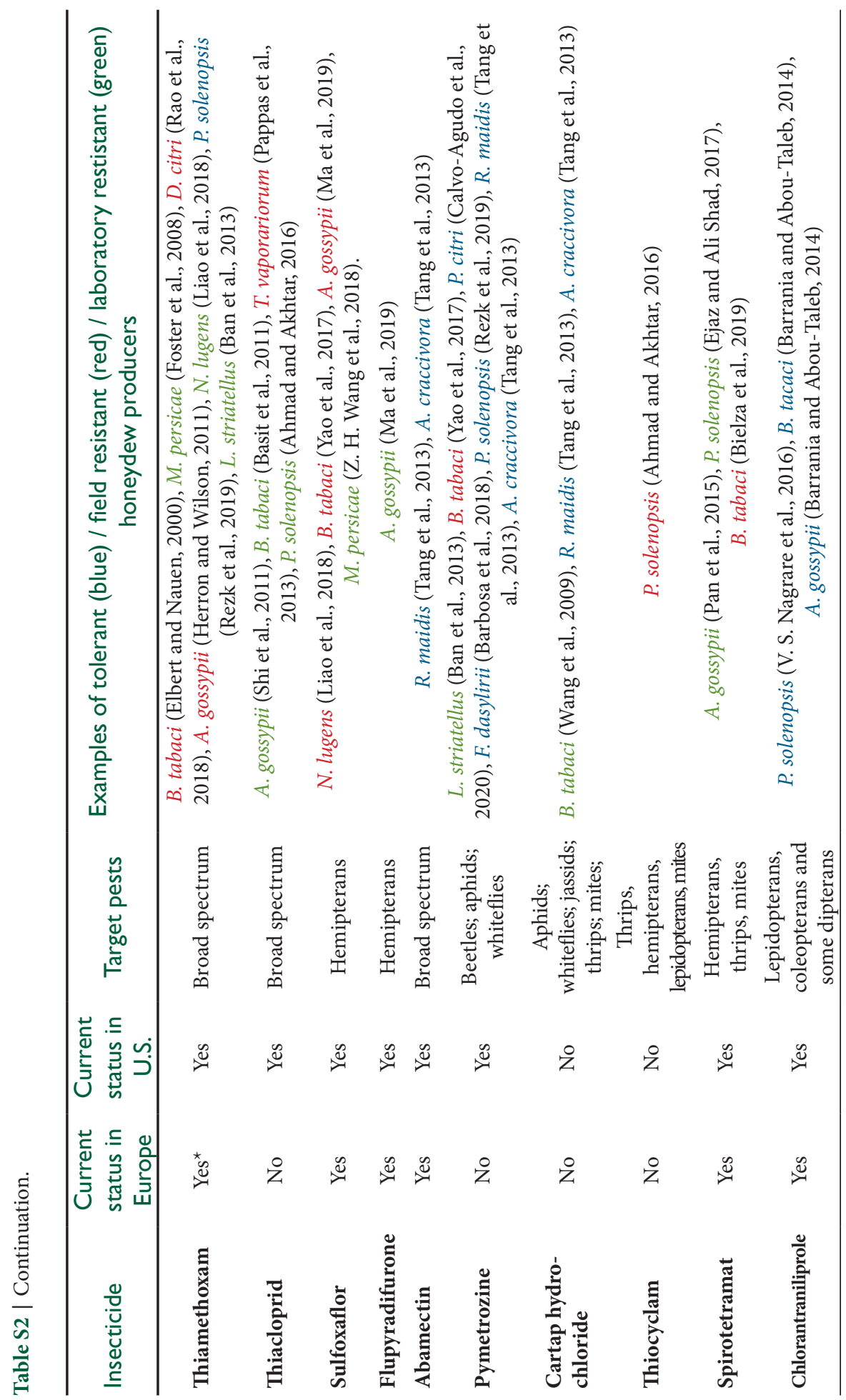




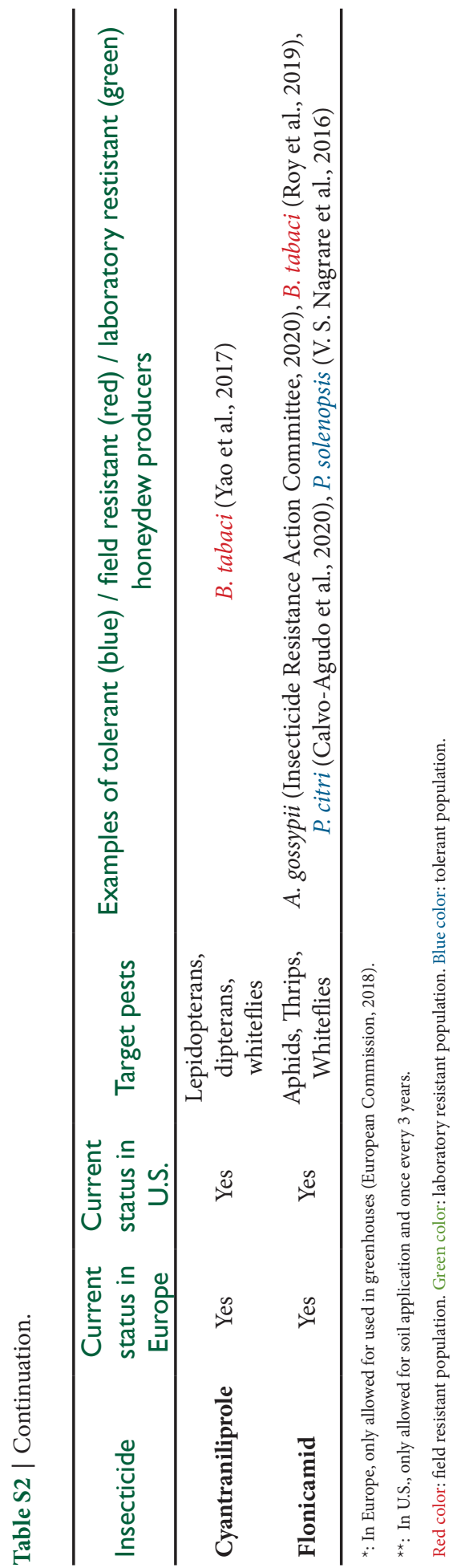





\section{Chapter 7}

General discussion
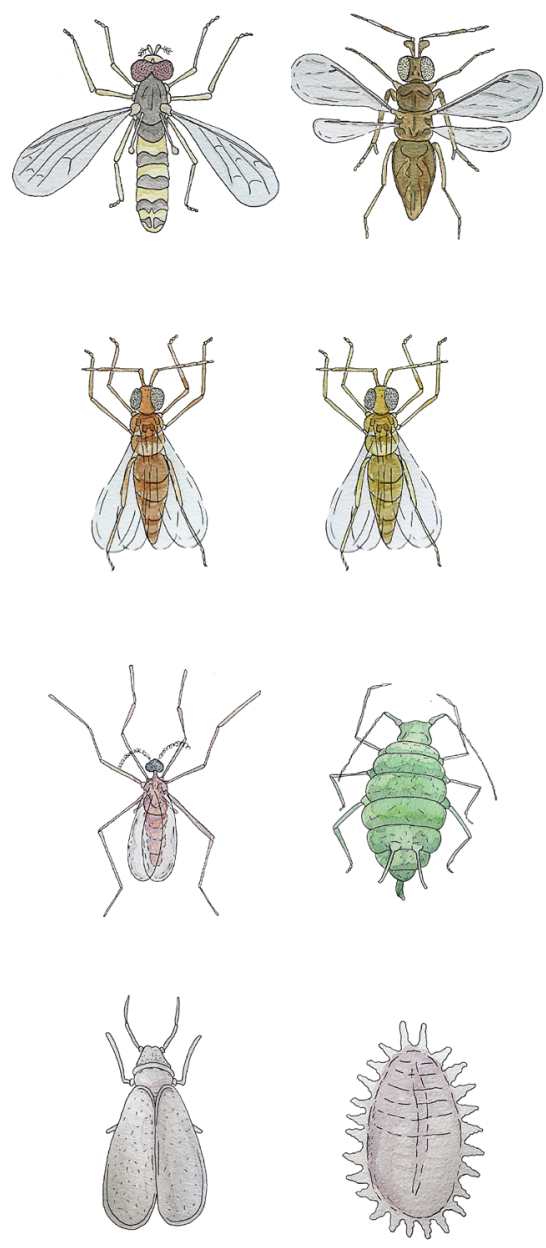

In agriculture, beneficial insects find carbohydrates mainly in floral and extrafloral nectar, guttation, fruit, or honeydew (Heimpel and Jervis, 2005; Lundgren, 2009; UrbanejaBernat et al., 2020; Wäckers et al., 2008, 2005). As explained extensively in this thesis, many of these carbohydrate sources are scarce in most agricultural landscapes. Nectar, for instance, is limited to the brief flowering period of the crop (if present), and to the blooming period of farmland flowers that appear along crop borders. Instead, honeydew is a ubiquitous carbohydrate source that is present during most of the growing season in many crops (Tena et al., 2016; Wäckers et al., 2008). It is well-known that beneficial insects feed on honeydew, either as a common food source as occurs with various species of parasitic wasps, predatory midges, and pollinators (Calabuig et al., 2015; Fratoni et al., 2019; Herrera, 1990; Hogervorst et al., 2007; Konrad et al., 2009; Lee et al., 2006; Lundgren, 2009; Luquet et al., 2021; Rogers et al., 2007; Tena et al., 2013a, 2016; Way, 1963), or as a substitute of nectar when the latter is scarce (Cameron et al., 2019; Konrad et al., 2009; Meiners et al., 2017).

Systemic insecticides are widely used to manage insect pests in agriculture. However, one disadvantage of using systemic insecticides is that plant-derived carbohydrate sources are usually contaminated via many different routes as explained in chapter $\mathbf{1}$ (section 4.2), and sugar-feeding beneficial insects are exposed to them (Goulson, 2013; Whitehorn et al., 2012; Woodcock et al., 2017). In this PhD project, I have discovered that the main carbohydrate in agriculture, honeydew, may be a frequent route of insecticide exposure that harms beneficial insects that feed on it. This has been demonstrated 
after studying: i) two crop species: citrus and soybean; ii) three honeydew-producing species from three different families: mealybugs, whiteflies and aphids; iii) four systemic insecticides: imidacloprid, thiamethoxam, flonicamid and pymetrozine, iv) three modes of application of systemic insecticides: foliar application, soil drench and seed coatings, and v) the toxicity of contaminated honeydew for five beneficial insect species: three parasitic wasps, a predatory midge, and a pollinator and predatory hoverfly (chapters 2, 3 and 4). In chapter 5, I have found that beneficial insects may not discriminate between honeydew contaminated with insecticides or uncontaminated honeydew. Finally, in chapter 6, I have opened this new area of research in ecotoxicology to research institutes and environmental protection agencies that can further study this route of exposure by exploring: i) potential pathways through which honeydew might be contaminated with insecticides, ii) which systemic insecticides that are more likely to reach honeydew, iii) which hemipteran species are more likely to excrete contaminated honeydew, and iv) some model scenarios in Europe and/or the U.S. where contaminated honeydew could be problematic for beneficial insects.

In this chapter, I discuss and connect the results of each chapter aiming to highlight the ecological and agricultural implications of this newly described route of insecticide exposure to beneficial insects. In detail, I address three major topics in which this route of exposure could be relevant nowadays: i) Invasive pests as honeydew producers; ii) New frameworks for environmental risk assessment; iii) Contaminated honeydew as a contributor to global insect decline. I end this general discussion with some concluding remarks on how this thesis contributes to society and science.

\section{INVASIVE PESTS AS HONEYDEW PRODUCERS}

Due to globalization, arthropod pests are increasingly invading new regions worldwide (Seebens et al., 2017). Many of these species, such as the soybean aphid Aphis glycines Matsumura (Hemiptera: Aphididae) that was used in chapter 4, excrete honeydew. As an example, the European and Mediterranean Plant Protection Organization (EPPO) has listed 39 invasive honeydew-producing species that may arrive soon or have recently arrived in Europe (Table 1). This list does not include unknown invasive pest species. For instance, in the last 15 years, 11 pests invaded Spanish citrus orchards, of which five excrete honeydew (personal observation of Alejandro Tena). Only one species of the five honeydew producing species, Trioza erytreae (Del Guercio) (Hemiptera: Triozidae), was included in the EPPO lists (Table 1). These invasive and honeydewproducing pests are generally treated with systemic insecticides until biological control strategies can be established (Frank and Tooker, 2020; GIP Citricos, 2021; Monzo and 


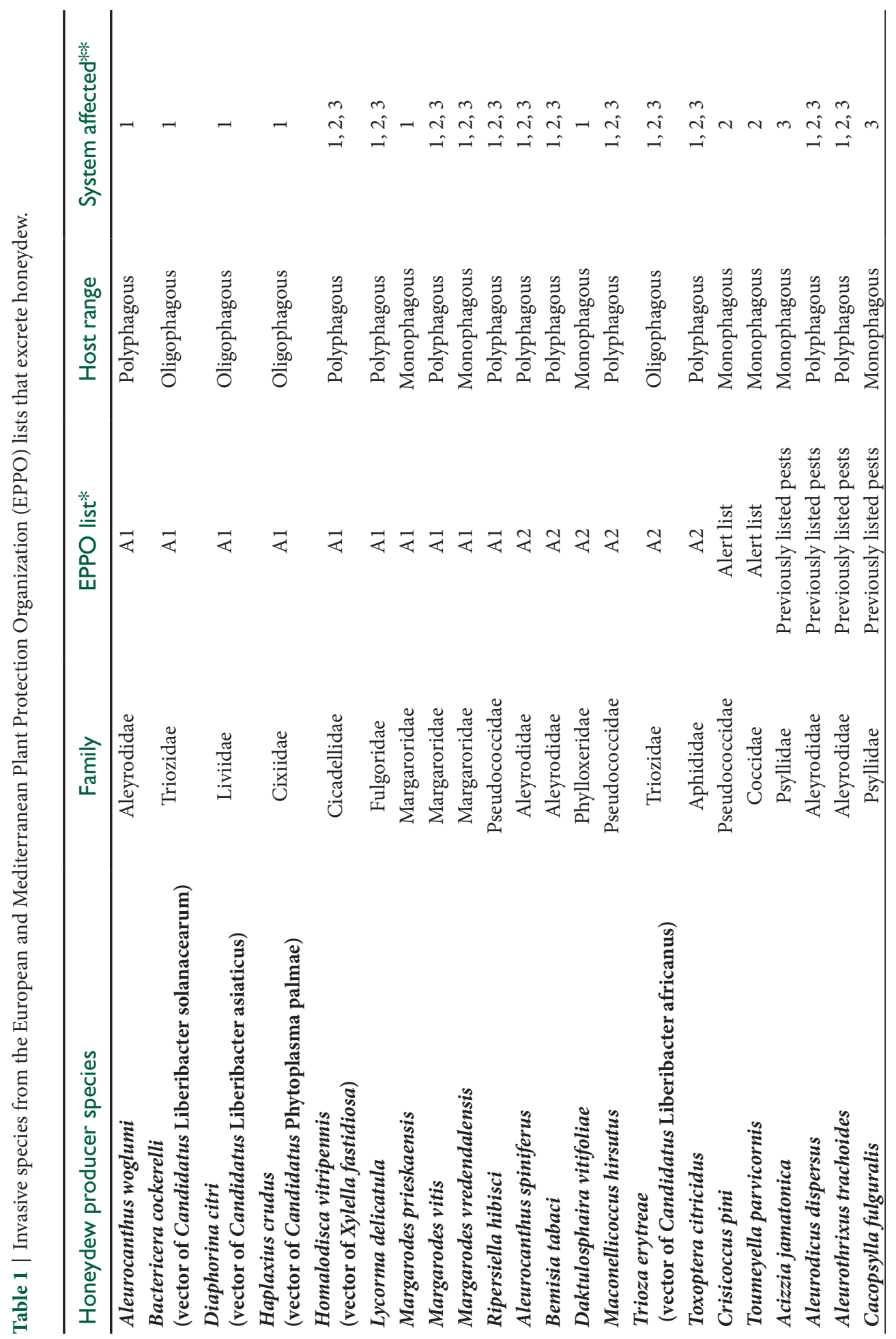




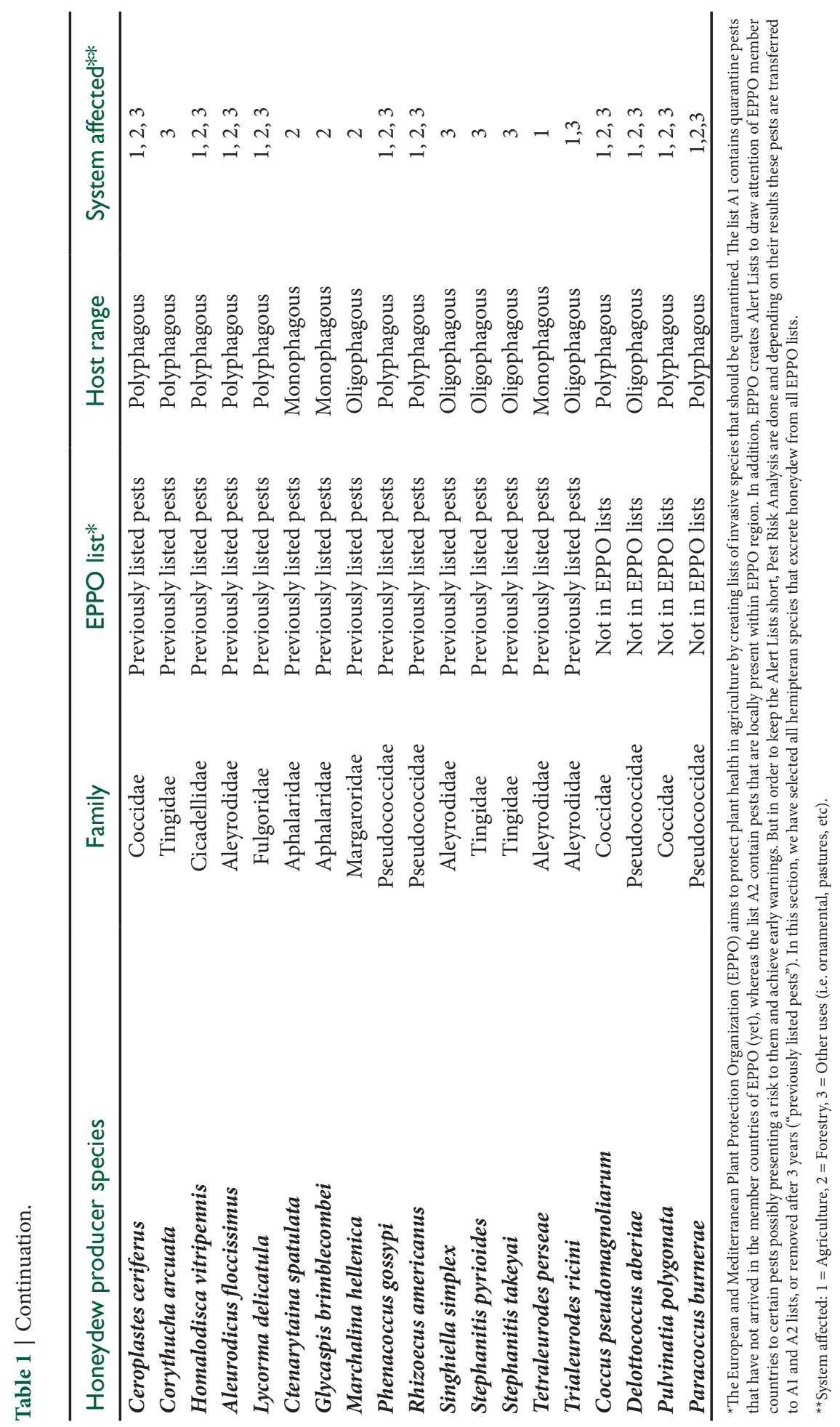


Stansly, 2017). For example, the mealybug Delottococcus aberiae De Lotto (Hemiptera: Pseudococcidae), which has recently invaded the Mediterranean citrus area (Beltra et al., 2015), is treated with systemic insecticides such as sulfoxaflor, acetamiprid and spirotetramat (GIP Citricos, 2021). Similarly, in the U.S., neonicotinoids are commonly applied against the Asian citrus psyllid Diaphorina citri Kuwayama (Hemiptera: Liviidae) in citrus, the soybean aphid in soybean or the polyphagous pest Lycorma deliculata (White) (Hemiptera: Fulgoridae) (Frank and Tooker, 2020; Leach et al., 2019; Monzo and Stansly, 2017). Therefore, applications with systemic insecticides and excretion of honeydew contaminated with these insecticides are likely to increase with the continuing introductions of exotic and invasive pest species in the coming years (Frank and Tooker, 2020). This thesis contributes to the understanding of how insecticide applications can indirectly impact beneficial insects when invasive species excrete honeydew contaminated with insecticides (chapter 4). Therefore, new strategies to control invasive pests while reducing the application of systemic insecticides are needed.

\section{I.I.A roadmap to reduce the use of systemic insecticides on invasive species}

When pests invade new areas, they arrive without their biological control agents that naturally control their populations in their country of origin (DeBach and Rosen, 1974). Therefore, chemical control, generally based on systemic insecticides, is used to manage invasive pest populations. At this point, alternative tools to reduce the number of invasive pest species and systemic insecticide applications are needed.

First, preventive measures need to be reinforced to avoid new invasive pests. For instance, national plant protection organizations (NPPO) of each importing and exporting country need to standardize protocols in order to prevent the introduction of invasive species. For instance, cold treatments or modified atmospheres are generally applied on the food containers during the transport to the importing country (Food and Agriculture Organization of the United Nations, 2018). However, some NPPO of importing countries do not receive the data to check whether the food containers travelled under the standard conditions established for the correct implementation of the treatment (Food Agriculture Organization, 2018).

Second, authorities should facilitate classical biological control programs because it is more economic than chemical control, and often it results in enormous economic benefits due to a permanent pest population reduction or eradication (Cock et al., 2010; van Lenteren et al., 2017). Over the past 120 years, introductions of more than 2,000 nonnative control agents in 196 countries or islands have been made with remarkably few 
environmental problems (Bale et al., 2008; Heimpel and Mills, 2017). Several constraints affect the implementation of classical biological control programs. The biology and biological control agents of the invasive pests are sometimes unknown and it takes time to gain sufficient knowledge to set up a classical biological control strategy. This is because some invasive pest species come from developing countries where the new invasive species does not have pest status and its biology and biological control agents are unknown. Establishing collaborations to study the biology of pests and their biological control agents with the countries of origin may ameliorate the speed of classical biological control programs.

Another constraint for the establishment of classical biological control is to comply with the requirements of the Nagoya Protocol. This protocol was created in 2014 with the objective of access and equitable sharing of benefits arising from the utilization of genetic resources, hence contributing to the conservation and sustainable use of biodiversity. The Nagoya Protocol aimed to create legal certainties and transparencies for both providers and users of genetic resources. To comply with the Nagoya Protocol, researchers who carry out classical biological control programs need to seek permissions to access the genetic resources at the planning stage, before collecting the biological control agents in the country of origin (Smith et al., 2018a). Nevertheless, obtaining these permissions is complicated, not transparent, and the information varies from one country to another. For all the above, facilitating the bureaucracy and creating common legislative framework among countries would reduce the amount of time and money invested in trying to understand how to import the natural enemies of the invasive pest (Smith et al., 2018b). This would facilitate biological control programs and reduce the amount of insecticides applied in agricultural lands.

Third, the application of the systems approaches within IPM frameworks may be key to control the establishment and spread of invasive species (Food and Agriculture Organization of the United Nations, 2017). Systems approaches integrate a set of tools to manage the risk of pests, thus providing an alternative to single measures and they might avoid the overuse of chemical control. At the pre-planning stage, one tool that may reduce the use of systemic insecticides to manage invasive pests is the use of hostplant resistant varieties (Heimpel et al., 2013). For instance, some soybean varieties that are resistant to the soybean aphid A. glycines have shown great potential to reduce insecticide use (Heimpel et al., 2013; Ragsdale et al., 2011). Moreover, the addition of flowering plants with high nectar content and diverse flowering periods in field margins and/or cover crops may enhance the efficacy of biological control agents by increasing 
their longevity and reproductive performance (Heimpel and Jervis, 2005; Landis et al., 2000). Other tools, such as the use of pheromones, have been implemented successfully for some invasive species. For instance, the sex pheromone of the invasive mealybug $D$. aberiae has been synthesized recently (Vacas et al., 2019), and may have great potential to reduce the populations of this mealybug.

\section{NEW FRAMEWORKS FOR ENVIRONMENTAL RISK ASSESSMENTS}

Environmental agencies such as the European Food Safety Authority (EFSA) and the U.S. Environmental Protection Agency (EPA) periodically carry out risk assessments to evaluate the safety of active ingredients. Risk assessments comprise Human and Ecological evaluations. Here, I will focus on environmental risk assessments (ERA) because the center of attention of this thesis is on the impact of honeydew on beneficial insects. ERA are necessary for making legal decisions regarding the usage of insecticides, both new and existing. One part of the current ERA is focused on evaluating the route of contamination of plant-derived food sources such as nectar, extrafloral nectar, or pollen (Gierer et al., 2019; Sanchez-Bayo and Goka, 2014). This thesis, in particular chapters 2, 3 and 4, provides information on a plant-derived food source, honeydew, which should be included in future ERA because it is a common food source in agroecosystems and forests (Calabuig et al., 2015; de-Miguel et al., 2014; Hogervorst et al., 2007; Lee et al., 2006; Luquet et al., 2021; Tena et al., 2013a; Ülgentürk et al., 2020) and it may be contaminated with systemic insecticides. In the following section, I explain why the safety of neonicotinoids and other insecticides with other mode of action than neonicotinoids should be reconsidered in future ERA. I also explain the importance of including more species in ERA than just a few surrogate species, i.e. non-target species representative for trophic levels in general (Hilbeck et al., 2011; Sanchez Bayo and Tennekes, 2017).

\section{I. Neonicotinoids}

Neonicotinoids have received most of the attention of this thesis because they are among the most widely used and toxic insecticides, accounting for more than $20 \%$ of the world's insecticide market in 2008 (Jeschke et al., 2011). Furthermore, the use of neonicotinoids is increasing, despite the scientific evidences of environmental harms demonstrated in literature (Douglas and Tooker, 2015; Tooker and Pearsons, 2021). Chapter 2 demonstrates, for the first time, that honeydew is a route of neonicotinoid exposure that harms beneficial insects that feed on it. Furthermore, chapter 4 shows that neonicotinoids reach honeydew excreted by aphids that are feeding on soybean plants whose seeds had been coated with neonicotinoids. 
Neonicotinoid seed coatings are the leading delivery method of neonicotinoid applications (Douglas and Tooker, 2015; Jeschke et al., 2011). However, this application method does not always provide economic benefits to farmers in the form of yield improvements (Bredeson and Lundgren, 2015; Labrie et al., 2020; Mourtzinis et al., 2019; Smith et al., 2020). For instance, two studies conducted in Canada found that neonicotinoids had the potential to protect soybean and corn yields in only less than $8 \%$ of the cases (Labrie et al., 2020; Smith et al., 2020). Even though yields rarely increase when using seed treatments, the use of neonicotinoids is now being reviewed by some environmental protection agencies. In the U.S., the EPA has recently scheduled a re-evaluation of neonicotinoid pesticides for 2022 (Environmental Protection Agency, 2021). In the European Union and UK, environmental agencies are also re-evaluating the use of neonicotinoid-coated seeds in crops that are harvested before the flowering period such as beets (European Commission, 2021; Government UK, 2021). Current evaluations have not considered that these plants can hold hemipterans that excrete contaminated honeydew during long periods, which can harm biological control agents. By including honeydew in future neonicotinoid ERA, environmental agencies will gain knowledge on the negative effects of insecticides on beneficial insects and the ecosystem services they provide.

\subsection{Systemic insecticides with other modes of action than neonicotinoids.}

A victory for conservation of biodiversity was thought when neonicotinoids were banned in Europe (Siviter and Muth, 2020). However, these restrictions to neonicotinoid applications will only benefit biodiversity if newer classes of insecticides are not toxic to non-target insect species. The toxic effects of other systemic insecticides such as pymetrozine, flonicamid, sulfoxaflor, flupyradifurone or spirotetramat have been less explored than that of neonicotinoids. Therefore, the goal of chapter 3 was to study whether non-neonicotinoids systemic insecticides reach honeydew and harm beneficial insects. We used flonicamid and pymetrozine because they are selective to whiteflies, aphids, planthoppers or leafhoppers but mealybugs are tolerant to them (El-Zahi et al., 2016; Rezk et al., 2019). Furthermore, in chapter 3, it was demonstrated that honeydew contaminated with flonicamid or pymetrozine is toxic for one of the two species of beneficial insect tested. For these reasons, honeydew should be included in next insecticide evaluations of all systemic insecticides taking into consideration the species of beneficial insects that they can reach. In these evaluations, all pathways of exposure should be contemplated (see point 2: "How can systemic insecticides reach honeydew" in chapter 6). 


\subsection{Mix of stressors or combination of pesticides}

Beneficial insects face many different stresses caused directly or indirectly by humans that include pesticides, climate change, pathogens, and habitat loss that causes depletion of their nutritional resources and nesting sites. Interaction between multiple stressors can exacerbate negative effects of pesticides (Ricupero et al., 2020; Siviter and Muth, 2020). For instance, the toxic effect of insecticides can be synergized when beneficial insects face hazardous temperatures (Ricupero et al., 2020). In addition, residues from multiple pesticides can be found in agroecosystems and plant-derived food sources (Azpiazu et al., 2019; David et al., 2016; Tosi and Nieh, 2019; Zioga et al., 2020).

Some fungicides can increase the toxicity of insecticides (Azpiazu et al., 2019; Sgolastra et al., 2017; Tosi and Nieh, 2019; Willow et al., 2019). One of the aims of chapter 4 was to determine whether honeydew excreted by aphids feeding on plants grown from seeds coated with a mix of fungicides and neonicotinoids was more toxic for beneficial insects than seeds coated only with neonicotinoids. In our study, honeydew derived from seeds coated with fungicides and neonicotinoids did not reduce the longevity of beneficial insects more than that of seeds coated only with neonicotinoids. However, other studies have demonstrated that the toxic effect of neonicotinoids is synergized by other fungicides than those used in chapter 4, such as tebuconazole (Willow et al., 2019) or propiconazole (Sgolastra et al., 2017; Tosi and Nieh, 2019). The differences in toxicities recorded in chapter 4 and those recorded in other studies may be attributed to the different physiochemical properties or mode of action of each active ingredient; mefenoxam is highly mobile in plants and soils, but has a low persistence in the environment that may make it safe to be used in IPM-programs (Camargo et al., 2019). Instead, tebuconazole and propiconazole have optimal translocation properties and high persistence in soil, water and plants. Therefore, it is likely that it reaches honeydew and synergize the toxic effect of neonicotinoids (Sgolastra et al., 2017; Willow et al., 2019).

In chapter 4, I aimed to explore other combinations of insecticides in seed coatings, but I could not do it because the experiments with the three treatments tested were already big for one person. For instance, soybean seeds may contain the insecticides thiamethoxam and abamectin, and two of the fungicides tested in chapter 4 (Avicta Complete Beans;, Syngenta). These seeds could have resulted in higher toxicity for beneficial insects than the seed coatings used in chapter $\mathbf{4}$ because abamectin can possibly reach hemipteran honeydew in addition to thiamethoxam. Abamectin has limited translocation properties (Dembilio et al., 2015; University of Herthfordshire, 2021) and hence, it is likely that it only reaches the first trifoliate leaves when plants are at their early developmental stages. 
In chapter 4, I collected honeydew contaminated with thiamethoxam when aphids were feeding on the first, second and third trifoliate leaves (V1-V3). Therefore, the limited systemic properties of abamectin could be enough to arrive to these trifoliate leaves and reach honeydew, as occurs with thiamethoxam. This honeydew may result more harmful for beneficial insects than the honeydew collected from aphids feeding on plants coated with thiamethoxam.

Some insecticides are highly persistent in soil, water and plants and highly mobile in water (see Table S2 in chapter 6 for properties). In some cases, they can be retained in soil for more than 10 years at low concentrations (Humann-Guilleminot et al., 2019), and may reach honeydew through many different pathways (See Table 5 in chapter 6), as they also reach nectar and pollen (Zioga et al., 2020). Low concentrations of an active ingredient may not be lethal for beneficial insects, but if they accumulate in the insect body, or they are combined with other active ingredients, they may harm them (Desneux et al., 2007; Goulson, 2013). Therefore, assessing the risk of different combinations of active ingredients that can be commonly found in agricultural landscapes is needed in future ERA.

\subsection{Number of species tested in ecotoxicological studies}

One of the main limitations of current ERA is that the entire framework is based on the acute toxicity of a few surrogate species. For instance, the honeybee Apis mellifera L. (Hymenoptera: Apidae) is the main insect species used in current ERA (Banks et al., 2014; Sanchez-Bayo and Goka, 2014). However, using one or few surrogate species to predict the fate of many species is widely variable (Banks et al., 2014). Also, the selection of organisms that should be included in each environmental test should be done on criteria including likeliness of being exposed and ecological importance of the species (Hilbeck et al., 2011). As an example, wild bee species and non-bee insects in ERA should be mandatory (Siviter and Muth, 2020) as they are often present in agricultural fields, and they are key to maintain yields of many crops by making $61 \%$ of the visits to crop flowers and sustaining life on Earth (Rader et al., 2015). In chapters 2 and 3, I used hoverflies because they are the most important non-bee pollinators (Rader et al., 2015). Sphaerophoria rueppellii (Wiedemann) (Diptera: Syrphidae) was evaluated in this Thesis because it is commonly found in the Mediterranean basin (Jiménez, 2013) and it is highly sensitive to insecticides (Abd-Ella, 2015; Jansen, 2000; Jansen et al., 2011; Moens et al., 2011). During the experiments of my thesis, I wanted to evaluate the toxicity of insecticides in honeydew using other pollinator species such as solitary bees or butterflies. I finally discarded this option because of the limited amount of honeydew and time to 
rear other species than those included in this thesis. With the honeydew that I collected, I could only evaluate the toxicity on three species and conduct chemical analyses to assess presence of insecticides and quantify their concentrations. Therefore, the toxic effects demonstrated for the hoverfly in chapter 2 and 3 should be complemented with further studies on other pollinator species, likely from other insect orders.

Furthermore, biological control agents are rarely included in ERA (Sanchez Bayo and Tennekes, 2017; Siviter and Muth, 2020). As for most pollinators, the ability to predict the fate of biological control agents in ERA depends exclusively on a simplistic extrapolation of data from the effects on acute toxicity on the honeybee (Banks et al., 2014). In chapters 2 and 4, I showed that honeydew contaminated with thiamethoxam was harmful for the parasitic wasps $A$. vladimiri and A. certus. These two parasitic wasps can be found commonly in citrus and soybean fields, respectively, they have a high ecological importance (Kaser and Heimpel, 2018; Noyes, 2021), but parasitic wasps have not been included in the ERA of neonicotinoids.

\subsection{Environmental fate of insecticides}

In addition to the evaluation of the toxicological profile of insecticides on non-target species, risk assessments study how insecticides move in the environment, and how they degrade in soil, water, light or within the plant. Chapter 5 aims to explore the routes of honeydew contamination, thus facilitating environmental protection agencies to regulate the use of pesticides according to their solubility in water and their persistence in the environment.

\section{CONTAMINATED HONEYDEW AS A CONTRIBUTOR TO GLOBAL INSECT DECLINE}

Insects are declining (Basset and Lamarre, 2019; Biesmeijer et al., 2006; Dirzo et al., 2014; Hallmann et al., 2021, 2017; Lister and Garcia, 2018). A growing number of studies provide evidence of declines in insect biomass and diversity across all functional groups i.e. herbivores, detritivores, biological control agents or pollinators (Harvey et al., 2020). The most important factors involving insect losses are habitat loss and fragmentation, invasive species and pathogens, climate change, and the use of pesticides such as those used in this thesis (Basset and Lamarre, 2019). However, the relative importance of each factor is still unknown. In this section, I will focus on how honeydew can play a role in insect decline when it is contaminated with insecticides. Honeydew is a hidden driver of direct and indirect interactions among insects (Moreno Ramírez, 2020), that indirectly impacts the population dynamics of herbivores, biological control agents, 
ants, and pollinators throughout different interactions such as mutualism, competition, commensalism, parasitism or predation (Moreno Ramírez, 2020; Tena et al., 2016). If honeydew is contaminated with insecticides as shown in chapters 2,3 and 4 , these interactions can be disrupted, thus altering trophic chains and ultimately, contributing to a decline of those insects that feed on contaminated honeydew (Kehoe et al., 2020).

\section{I. Honeydew mediating insect decline in agricultural landscapes}

With the intensification of modern agriculture to increase food production, more than six million tons of pesticides are applied every year, of which 600,000 tons are insecticides (Bernhardt et al., 2017; Zhang, 2018). A large amount of these insecticide applications corresponds to systemic insecticides, which are often highly toxic to nontarget insects. As an example, from summer 2010 to fall 2013, 31 insecticide applications were applied against the Asian citrus psyllid, D. citri in Florida (U.S.) (Monzo and Stansly, 2017). From these 31 applications, thirteen corresponded to systemic insecticides that included carbamates, organophosphates, neonicotinoids or tetronic and tetramic acid derivatives. Several hemipteran species may concurrently inhabit citrus orchards (Tena et al., 2013b) and excrete the systemic insecticides when the latter are applied against $D$. citri. In this scenario, honeydew-feeding insects can be exposed to one or more systemic insecticides applied to the target crop plant (See Figure $2 \mathrm{a}, \mathrm{b}, \mathrm{c}$ in chapter 6), or to adjacent non-target plants (See Figure 2 d, e, f, in chapter 6). For instance, $70-100 \%$ of the hoverflies, and 60-65\% of the parasitic wasps died when they were fed on honeydew excreted by mealybugs feeding on soil- or foliar-treated trees with thiamethoxam (chapter 2). Similarly, 22 and 56\% of the hoverflies died when they were fed on honeydew contaminated with pymetrozine and flonicamid, respectively (chapter 3 ). Therefore, honeydew contaminated with insecticides may be directly correlated with insect decline in those species that feed on honeydew.

The European environmental protection agencies are re-evaluating the use of neonicotinoid-coated seeds for emergency authorizations in beet crops (Syngenta's Cruiser SB) (European Commission, 2021; Government UK, 2021), a scenario that is likely to have negative effects on insect biomass and biodiversity (European Commission, 2021; Government UK, 2021). Beets are harvested before the flowering period and thereby, the risk for beneficial insects that will feed on nectar of beet plants has been categorized as acceptably low (Government UK, 2021). However, a higher risk might be expected when water-soluble neonicotinoids move to the nectar of flowering plants in and around the crop. To minimize this risk, the UK authorities, in accordance to the applicants, has recommended: i) herbicide programs to avoid flowering weeds surrounding treated 
beet crops; and ii) to ensure that no flowering crops are planted for a period of more than 22 months (or 32 months if this crop is oilseed rape) (Government UK, 2021). The application of these recommendations would turn huge surfaces of agricultural land (beets were planted in 108,000 hectare only in UK in 2019 according to FAOSTAT) into inhospitable areas for biological control agents and pollinators. In these agroecosystems, which would be depleted of nectar, beneficial insects would have to rely on honeydew excreted by aphids left as its main carbohydrate source. As I have demonstrated in chapter 4 for soybean, honeydew excreted by aphids feeding on beets grown from coated-seeds may be contaminated with neonicotinoids and be toxic for biological control agents, thus contributing to insect decline.

\subsection{Honeydew mediating insect decline in non-agricultural habitats}

Water-soluble systemic insecticides are transported from agricultural lands to adjacent habitats or ecosystems by movement in water or by insecticide drift (Greatti et al., 2006; Krupke et al., 2012; Pearsons et al., 2021; Tsvetkov et al., 2017). For instance, a study detected imidacloprid in $90 \%$ of the water samples collected from agricultural regions of California (Starner and Goh, 2012). In 20\% of the cases, concentrations exceeded the U.S. EPA chronic invertebrate Aquatic Life Benchmarks of $1.05 \mu \mathrm{g} / \mathrm{L}$. Similarly, the carbamate pirimicarb was found in more than $50 \%$ of the samples of surface water investigated in Ontario, Canada (Natale et al., 2018; Struger et al., 2016). Through water, systemic insecticides reach habitats such as nature reserves, abandoned areas, or forests (Frank and Tooker, 2020; Goulson, 2013). In this scenario, the insecticides can be absorbed by plants and ingested and excreted by hemipterans (See Figure $2 \mathrm{~d}, \mathrm{e}, \mathrm{f}$ in chapter 6). In the literature, we can find an example in which a forestry hemipteran pest, the striped pine scale Toumeyella pini (King) (Hemiptera: Coccidae), can excrete spirotetramat and imidacloprid when feeding on plants that contain those insecticides (Quesada et al., 2020). Furthermore, one of the most important undesirable effects of systemic insecticides on beneficial insects can be explained by their physiochemical properties. Persistent insecticides such as neonicotinoids can remain in the soil and plant for years at low concentrations (Byrne et al., 2014; Humann-Guilleminot et al., 2019). Some hemipterans may tolerate low insecticide concentrations in the plant and traces of insecticides may travel unnoticed to the honeydew (chapter 6). This honeydew with insecticides might cause a range of sublethal effects to numerous insect species through accumulation (Goulson, 2013). If we extrapolate the results obtained in this thesis, with the high-water solubility, mobility and persistence of some insecticides, the results may partly explain why honeydew contaminated with insecticides may be another factor contributing to insect decline. 


\section{CONCLUDING REMARKS ON HOW THIS THESIS CONTRIBUTES TO SCIENCE AND SOCIETY}

Modern agriculture relies heavily on the use of pesticides against invasive and cosmopolitan pests (Frank and Tooker, 2020; Sánchez-Bayo et al., 2013). Some pesticides are highly persistent and move through the water and as dust particles to other environments, reaching beneficial insects throughout different routes of exposure (Frank and Tooker, 2020; Goulson, 2013). This thesis contributes to science by discovering a new route of insecticide exposure that has never been included in ERA. In most agroecosystems, this route may be more accessible for some beneficial insects than the well-studied route of floral nectar. This new route has been proven for different: i) crop species, ii) hemipteran species, iii) active ingredients, iv) modes of application, and v) beneficial insect species. In addition, I have opened this area of research to other scientists and environmental protection agencies by studying the pathways of exposure through which honeydew might be contaminated with insecticides and the systemic insecticides that may reach honeydew more frequently. The results of this thesis may help further understanding of the effects of systemic insecticides on nature. For instance, honeydew could be included in the Worldwide Integrated Assessment on the risks of Systemic Pesticides to Biodiversity and Ecosystems (WIA) that has recently reviewed the risks of systemic insecticides (van Lexmond et al., 2015). In fact, this route, which was neglected in the WIA, could help having a more complete view of the effects of systemic insecticides on insect decline.

Science brings knowledge to society. By including honeydew in future environmental evaluations of insecticides, society may benefit of more sustainable agricultural policies that may allow us to produce food without compromising future generations. 


\section{References}
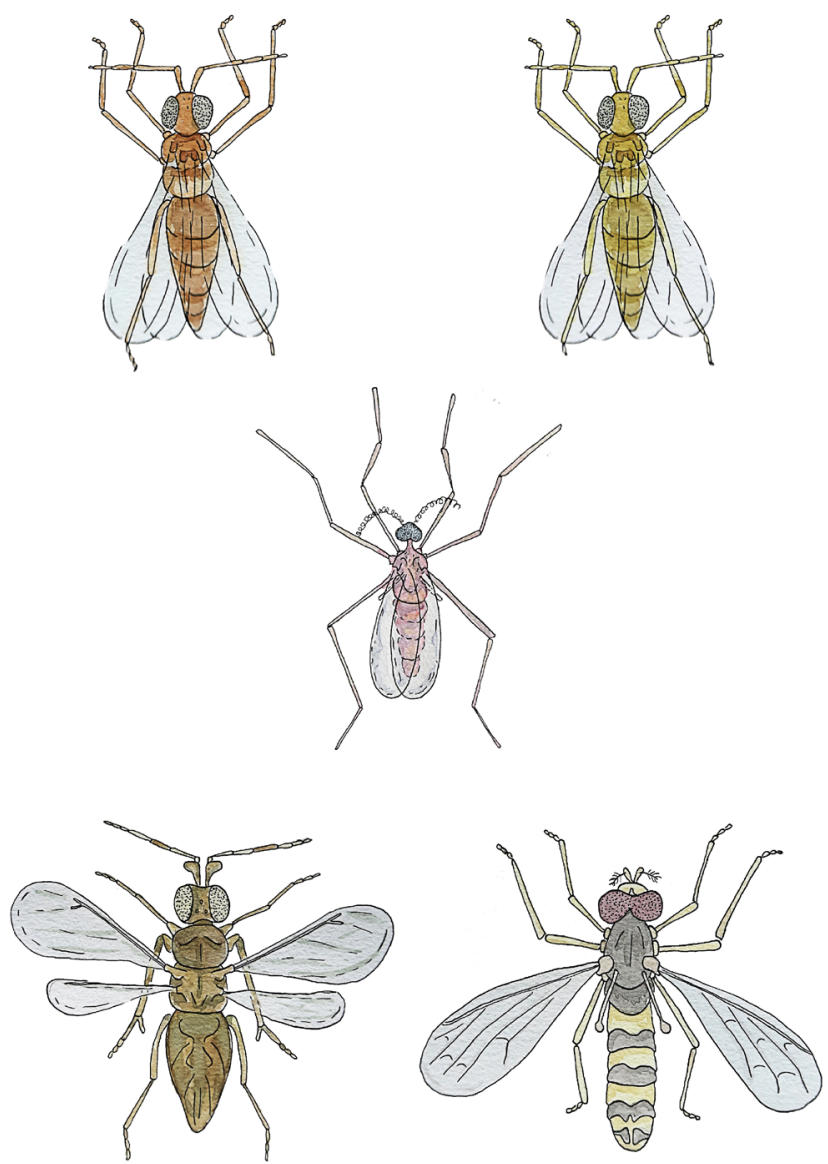

Abd-Ella, A.A., 2015. Effect of several insecticides on pomegranate aphid, Aphis punicae (Passerini) (Homoptera: Aphididae) and its predators under field conditions. Bull. EPPO 45, 90-98. https://doi.org/10.1111/epp.12192

Abdourahime, H., Arena, M., Auteri, D., Barmaz, S., Ctverackova, L., De Lentdecker, C., Ippolito, A., Kardassi, D., Messinetti, S., Molnar, T., Saari, K.E., Sharp, R., Streissl, F., Sturma, J., Szentes, C., Tiramani, M., Vagenende, B., Van Dijk, J., Villamar-Bouza, L., 2019. Peer review of the pesticide risk assessment for the active substance sulfoxaflor in light of confirmatory data submitted. EFSA J. 17, 5633. https://doi.org/10.2903/j.efsa.2019.5633

Afzal, M.B.S., Shad, S.A., Abbas, N., Ayyaz, M., Walker, W.B., 2015. Cross-resistance, the stability of acetamiprid resistance and its effect on the biological parameters of cotton mealybug, Phenacoccus solenopsis (Homoptera: Pseudococcidae), in Pakistan. Pest Manag. Sci. 71, 151-158. https://doi.org/10.1002/ps.3783

Ahmad, M., Akhtar, S., 2016. Development of resistance to insecticides in the invasive mealybug Phenacoccus solenopsis (Hemiptera: Pseudococcidae) in Pakistan. Crop Prot. 88, 96-102. https://doi.org/10.1016/j.cropro.2016.06.006

Ahmad, M., Arif, M.I., Ahmad, Z., Denholm, I., 2002. Cotton whitefly (Bemisia tabaci) resistance to organophosphate andpyrethroid insecticides in pakistan. Pest Manag. Sci. 58, 203-208. https://doi.org/10.1002/ps.440

Ahmad, M., Iqbal Arif, M., 2008. Susceptibility of Pakistani populations of cotton aphid Aphis gossypii (Homoptera: Aphididae) to endosulfan, organophosphorus and carbamate insecticides. Crop Prot. 27, 523-531. https://doi.org/10.1016/j. cropro.2007.08.006 
Aliniazee, M.T., 1983. Carbaryl resistance in the filbert aphid (Homoptera: Aphididae). J. Econ. Entomol. 76, 1002-1004. https://doi.org/10.1093/jee/76.5.1002

Ammar, E.D., Alessandro, R., Shatters, R.G., Hall, D.G., 2013. Behavioral, ultrastructural and chemical studies on the honeydew and waxy secretions by nymphs and adults of the Asian Citrus Psyllid Diaphorina citri (Hemiptera: Psyllidae). PLoS One 8, e64938. https://doi.org/10.1371/journal.pone.0064938

Amoros-Jiménez, R., 2013. Biología, interacciones y uso del depredador Sphaerophoria rueppellii (Diptera: Syrphidae) en el Control Integrado de plagas de áfidos en cultivos de invernadero. $\mathrm{PhD}$ thesis, Universidad de Alicante. Alicante, Spain.

Amorós-Jiménez, R., Pineda, A., Fereres, A., Marcos-García, M.Á., 2014. Feeding preferences of the aphidophagous hoverfly Sphaerophoria rueppellii affect the performance of its offspring. BioControl 59, 427-435. https://doi.org/10.1007/ s10526-014-9577-8

Anderson, N.L., Harmon-Threatt, A.N., 2019. Chronic contact with realistic soil concentrations of imidacloprid affects the mass, immature development speed, and adult longevity of solitary bees. Sci. Rep. 9, 3724. https://doi.org/10.1038/s41598-01940031-9

Arce, A.N., Rodrigues, A.R., Yu, J., Colgan, T.J., Wurm, Y., Gill, R.J., 2018. Foraging bumblebees acquire a preference for neonicotinoid-treated food with prolonged exposure. Proc. R. Soc. B Biol. Sci. 285, 20180655. https://doi.org/10.1098/ rspb.2018.0655

Azpiazu, C., 2020. Evaluation of Flowering Plant strips and the risk of pesticides on pollinators in melon agro-ecosystems. PhD thesis at Universidad Politécnica de Madrid. Madrid, Spain

Azpiazu, C., Bosch, J., Viñuela, E., Medrzycki, P., Teper, D., Sgolastra, F., 2019. Chronic oral exposure to field- realistic pesticide combinations via pollen and nectar : effects on feeding and thermal performance in a solitary bee. Sci. Rep. 9, 13770. https://doi. org/10.1038/s41598-019-50255-4

Baker, H.G., Opler, P.A., Baker, I., 1978. A comparison of the amino acid complements of floral and extrafloral nectars. Bot. Gaz. 139, 322-332. https://doi.org/10.1007/9783-540-36849-6_2

Bale, J.S., Van Lenteren, J.C., Bigler, F., 2008. Biological control and sustainable food production. Philos. Trans. R. Soc. B Biol. Sci. 363, 761-776. https://doi.org/10.1098/ rstb.2007.2182

Ban, L., Zhang, S., Huang, Z., He, Y., Peng, Y., Gao, C., 2013. Resistance monitoring and assessment of resistance risk to pymetrozine in Laodelphax striatellus (Hemiptera: Delphacidae). J. Econ. Entomol. 105, 2129-2135. https://doi.org/10.1603/ec12213 
Banks, John E., Stark, J.D., Vargas, R.I., Ackleh, A.S., 2014. Deconstructing the surrogate species concept: A life history approach to the protection of ecosystem services. Ecol. Appl. 24, 770-778. https://doi.org/10.1890/13-0937.1

Barber, M.D., Moores, G.D., Tatchell, G.M., Vice, W.E., Denholm, I., 1999. Insecticide resistance in the currant-lettuce aphid, Nasonovia ribisnigri (Hemiptera: Aphididae) in the UK. Bull. Entomol. Res. 89, 17-23. https://doi.org/10.1017/s0007485399000036

Barbosa, P.R.R., Oliveira, M.D., Barros, E.M., Michaud, J.P., Torres, J.B., 2018. Differential impacts of six insecticides on a mealybug and its coccinellid predator. Ecotoxicol. Environ. Saf. 147, 963-971. https://doi.org/10.1016/j.ecoenv.2017.09.021

Barrania, A.A., Abou-Taleb, H.K., 2014. Field efficiency of some insecticide treatments against whitefly, Bemisia tabaci, cotton aphid, Aphis gossypii and their associated predator, Chrysopa vulgaris, in cotton plants. Alex. J. Agric. Res 59, 105-111.

Basit, M., Sayyed, A.H., Saleem, M.A., Saeed, S., 2011. Cross-resistance, inheritance and stability of resistance to acetamiprid in cotton whitefly, Bemisia tabaci Genn (Hemiptera: Aleyrodidae). Crop Prot. 30, 705-712. https://doi.org/10.1016/j. cropro.2011.02.020

Basset, Y., Lamarre, G.P.A., 2019. Toward a world that values insects: Rapid adoption of conservation measures is key to protecting insect populations. Science 364, 12301231. https://doi.org/10.1126/science.aaw7071

Bayer Crop Science,2019. Imidacloprid (Confidor). URL https://www.cropscience.bayer. es/Productos/Insecticides/Confidor-20-LS.aspx.

Bayer Crop Science, 2020. Spirotetramat (Movento). URL https://www.cropscience. bayer.es/Productos/Insecticides/Movento-150-O-Teq

Belchim, 2021. Flonicamid (Teppeki). URL http://www.belchim.es/index.php/teppeki.

Bell, H.A., Kirkbride-Smith, A.E., Marris, G.C., Edwards, J.P., Gatehouse, A.M.R., 2004. Oral toxicity and impact on fecundity of three insecticidal proteins on the gregarious ectoparasitoid Eulophus pennicornis (Hymenoptera: Eulophidae). Agric. For. Entomol. 6, 215-222. https://doi.org/10.1111/j.1461-9555.2004.00225.x

Beltra, A., Addison, P., Ávalos, J.A., Crochard, D., Garcia-Marí, F., Guerrieri, E., Giliomee, J.H., Malausa, T., Navarro-Campos, C., Palero, F., Soto, A.S., 2015. Guiding classical biological control of an invasive mealybug using integrative taxonomy. PLoS One 10, e0128685. https://doi.org/10.1371/journal.pone.0128685

Berndt, L.A., Wratten, S.D., 2005. Effects of alyssum flowers on the longevity, fecundity, and sex ratio of the leafroller parasitoid Dolichogenidea tasmanica. Biol. Control 32, 65-69. https://doi.org/10.1016/j.biocontrol.2004.07.014

Bernhardt, E.S., Rosi, E.J., Gessner, M.O., 2017. Synthetic chemicals as agents of global change. Front. Ecol. Environ. 15, 84-90. https://doi.org/10.1002/fee.1450 
Bielza, P., Moreno, I., Belando, A., Grávalos, C., Izquierdo, J., Nauen, R., 2019. Spiromesifen and spirotetramat resistance in field populations of Bemisia tabaci Gennadius in Spain. Pest Manag. Sci. 75, 45-52. https://doi.org/10.1002/ps.5144

Biesmeijer, J.C., Roberts, S.P.M., Reemer, M., Ohlemüller, R., Edwards, M., Peeters, T., Schaffers, A.P., Potts, S.G., Kleukers, R., Thomas, C.D., Settele, J., Kunin, W.E., 2006. Parallel declines in pollinators and insect-pollinated plants in Britain and the Netherlands. Science 313, 351-354. https://doi.org/10.1126/science.1127863

Biondi, A., Guedes, R.N.C., Wan, F.-H., Desneux, N., 2018. Ecology, worldwide spread, and management of the invasive South American tomato pinworm, Tuta absoluta: past, present, and future. Annu. Rev. Entomol. 63, 239-258. https://doi.org/10.1146/ annurev-ento-031616-034933

Boina, D.R., Bloomquist, J.R., 2015. Chemical control of the Asian citrus psyllid and of huanglongbing disease in citrus. Pest Manag. Sci. 71, 808-823. https://doi.org/10.1002/ ps.3957

Bollard, E.G., 1960. Transport in the Xylem. Annu. Rev. Plant Physiol. 11, 141-166. https://doi.org/10.1146/annurev.pp.11.060160.001041

Bonmatin, J.M., Giorio, C., Girolami, V., Goulson, D., Kreutzweiser, D.P., Krupke, C., Liess, M., Long, E., Marzaro, M., Mitchell, E.A., Noome, D.A., Simon-Delso, N., Tapparo, A., 2015. Environmental fate and exposure; neonicotinoids and fipronil. Environ. Sci. Pollut. Res. 22, 35-67. https://doi.org/10.1007/s11356-014-3332-7

Botías, C., David, A., Horwood, J., Abdul-Sada, A., Nicholls, E., Hill, E., Goulson, D., 2015. Neonicotinoid residues in wildflowers, a potential route of chronic exposure for bees. Environ. Sci. Technol. 49, 12731-12740. https://doi.org/10.1021/acs.est.5b03459

Boulanger, F.X., Jandricic, S., Bolckmans, K., Wäckers, F.L., Pekas, A., 2019. Optimizing aphid biocontrol with the predator Aphidoletes aphidimyza, based on biology and ecology. Pest Manag. Sci. 75, 1479-1493. https://doi.org/10.1002/ps.5270

Boxall, A.B., Sinclair, C.J., Fenner, K., Kolpin, D., Maund, S.J., 2004. Peer reviewed: when synthetic chemicals degrade in the environment. Environ. Sci. Technol. 369-375.

Bredeson, M.M., Lundgren, J.G., 2015. Thiamethoxam seed treatments have no impact on pest numbers or yield in cultivated sunflowers. J. Econ. Entomol. 108, 2665-2671. https://doi.org/10.1093/jee/tov249

Bromilow, R.H., Chamberlain, K., 1991. Pathways and mechanisms of transport of herbicides in plants. Target Sites Herbic. Action 245-284. https://doi.org/10.1007/9781-4899-2433-9_9

Bromilow, R.H., Chamberlain, K., Evans, A.A., 1990. Physicochemical aspects of phloem translocation of herbicides. Weed Sci. Soc. Am. 38, 305-314. 
Brown, J.K., Forhlich, D.R., Rosell, R.C., 1995. The sweetpotato or silverleaf whiteflies: biotypes of Bemisia tabaci or a species complex? Annu. Rev. Entomol. 40, 511-534. https://doi.org/10.1146/annurev.ento.40.1.511

Budenberg, W.J.\& P.W., 1992. The role of honeydew as an ovipositional stimulant for syrphids. Entomol. Exp. Appl. 64, 57-61. https://doi.org/10.1111/j.1570-7458.1992. tb01594.x

Budenberg, W.J., 1990. Honeydew as a contact kairomone for aphid parasitoids. Entomol. Exp. Appl. 55, 139-148. https://doi.org/10.1111/j.1570-7458.1990.tb01357.x

Byrne, F.J., Urena, A.A., Robinson, L.J., Krieger, R.I., Doccola, J., Morse, J.G., 2012. Evaluation of neonicotinoid, organophosphate and avermectin trunk injections for the management of avocado thrips in California avocado groves. Pest Manag. Sci. 68, 811-817. https://doi.org/10.1002/ps.2337

Byrne, F.J., Visscher, P.K., Leimkuehler, B., Fischer, D., Grafton-Cardwell, E.E., Morse, J.G., 2014. Determination of exposure levels of honey bees foraging on flowers of mature citrus trees previously treated with imidacloprid. Pest Manag. Sci. 70, 470482. https://doi.org/10.1002/ps.3596

Cahill, M.R., Macey, M.G., Dawson, J.R., Newland, A.C., 1996. Platelet surface activation antigen expression at baseline and during elective angioplasty in patients with mild to moderate coronary artery disease. Blood Coagul. Fibrinolysis 7, 165-168. https://doi. org/10.1097/00001721-199603000-00013

Calabuig, A., Tena, A., Wäckers, F.L., Fernández-Arrojo, L., Plou, F.J., Garcia-Marí, F., Pekas, A., 2015. Ants impact the energy reserves of natural enemies through the shared honeydew exploitation. Ecol. Entomol. 40, 687-695. https://doi.org/10.1111/ een. 12237

Calatayud-Vernich, P., Calatayud, F., Simó, E., Morales Suarez-Varela, M., Picó, Y., 2016. Influence of pesticide use in fruit orchards during blooming on honeybee mortality in 4 experimental apiaries. Sci. Total Environ. 541, 33-41. https://doi.org/10.1016/j. scitotenv.2015.08.131

Caldwell, D.L., Gerhardt, K.O., 1986. Chemical analysis of peach extrafloral nectary exudate. Phytochemistry 25, 411-413. https://doi.org/10.1016/S0031-9422(00)85491-6

Calvo-Agudo, M., Dregni, J., González-Cabrera, J., Dicke, M., Heimpel, G.E., Tena, A., 2021. Neonicotinoids from coated seeds toxic for honeydew-feeding biological control agents. Environ. Pollut. 289, 117813. https://doi.org/10.1016/j.envpol.2021.117813

Calvo-Agudo, M., González-Cabrera, J., Picó, Y., Calatayud-Vernich, P., Urbaneja, A., Dicke, M., Tena, A., 2019. Neonicotinoids in excretion product of phloem-feeding insects kill beneficial insects. Proc. Natl. Acad. Sci. U.S.A. 116, 16817-16822. https:// doi.org/10.1073/pnas.1904298116 
Calvo-Agudo, M., González-Cabrera, J., Sadutto, D., Picó, Y., Urbaneja, A., Dicke, M., Tena, A., 2020. IPM-recommended insecticides harm beneficial insects through contaminated honeydew. Environ. Pollut. 267, 115581. https://doi.org/10.1016/j.envpol.2020.115581

Camargo, C., 2016. Ecological risks of the conventional insecticide/fungicide seed treatment mixture of thiamethoxam and mefenoxam in soybean on beneficial insects. $\mathrm{PhD}$ thesis, University of Nebraska - Lincoln. Nebraska, U.S.A.

Camargo, C., Snow, D.D., Onanong, S., Hunt, T.E., Siegfried, B.D., 2019. Residues of thiamethoxam and mefenoxam in vegetative and floral tissue of soybean at the early reproductive stage resulting from seed treatments. Crop Prot. 119, 134-140. https:// doi.org/10.1016/j.cropro.2019.01.019

Cameron, S.A., Corbet, S.A., Whitfield, J.B., 2019. Bumble bees (Hymenoptera: Apidae: Bombus terrestris) collecting honeydew from the giant willow aphid (Hemiptera: Aphididae). J. Hymenopt. Res. 68, 75-83. https://doi.org/10.3897/jhr.68.30495

Castañé, C., van der Blom, J., Nicot, P.C., 2020. Tomatoes, in: Gullino, M.L., Albajes, R., Nicot, P.C., Integrated Pest and Disease Management in Greenhouse Crops. pp. 487-511. https://doi.org/10.1016/s0168-9452(00)00461-1

Cen, Y., Yang, C., Holford, P., Beattie, G.A.C., Spooner-Hart, R.N., Liang, G., Deng, X., 2011. Feeding behaviour of the Asiatic citrus psyllid, Diaphorina citri, on healthy and huanglongbing-infected citrus. Entomol. Exp. Appl. 143, 13-22. https://doi. org/10.1111/j.1570-7458.2012.01222.x

Chen, M., Han, Z., Qiao, X., Qu, M., 2007. Resistance mechanisms and associated mutations in acetylcholinesterase genes in Sitobion avenae (Fabricius). Pestic. Biochem. Physiol. 87, 189-195. https://doi.org/10.1016/j.pestbp.2006.07.009

Chen, X., Meng, Z., Zhang, Y., Gu, H., Ren, Y., Lu, C., 2016. Degradation kinetics and pathways of spirotetramat in different parts of spinach plant and in the soil. Environ. Sci. Pollut. Res. 23, 15053-15062. https://doi.org/10.1007/s11356-016-6665-6

Cheng, S., Lin, R., Wang, L., Qiu, Q., Qu, M., Ren, X., Zong, F., Jiang, H., Yu, C., 2018. Comparative susceptibility of thirteen selected pesticides to three different insect egg parasitoid Trichogramma species. Ecotoxicol. Environ. Saf. 166, 86-91. https://doi. org/10.1016/j.ecoenv.2018.09.050

Choh, Y., Takabayashi, J., 2006. Herbivore-induced extrafloral nectar production in lima bean plants enhanced by previous exposure to volatiles from infested conspecifics. J. Chem. Ecol. 32, 2073-2077. https://doi.org/10.1007/s10886-006-9130-Z

Choi, M.-Y., Roitberg, B.D., Shani, A., Raworth, D.A., Lee, G.H., 2004. Olfactory response by the aphidophagous gall midge, Aphidoletes aphidimyza to honeydew from green peach aphid, Myzus persicae. Entomol. Exp. Appl. 111, 37-45. https://doi.org/10.1111/ j.0013-8703.2004.00151.x 
Chowdhury, A.Z., Jahan, S.A., Islam, M.N., Moniruzzaman, M., Alam, M.K., Zaman, M.A., Karim, N., Gan, S.H., 2012. Occurrence of organophosphorus and carbamate pesticide residues in surface water samples from the Rangpur district of Bangladesh. Bull. Environ. Contam. Toxicol. 89, 202-207. https://doi.org/10.1007/s00128-0120641-8

Clem, C.S., Sparbanie, T.M., Luro, A.B., \& Harmon-Threatt, A.N., 2020. Can anthophilous hover flies (Diptera: Syrphidae) discriminate neonicotinoid insecticides in sucrose solution?. PloS One 15, e0234820.

Clout, M.N., Gaze, P.D., 1984. Effects of plantation forestry on birds in New Zealand. J. Appl. Ecol. 21, 795-815. https://doi.org/10.2307/2405048

Cock, M.J.W., van Lenteren, J.C., Brodeur, J., Barratt, B.I.P., Bigler, F., Bolckmans, K., Cônsoli, F.L., Haas, F., Mason, P.G., Parra, J.R.P., 2010. Do new access and benefit sharing procedures under the convention on biological diversity threaten the future of biological control? BioControl 55, 199-218. https://doi.org/10.1007/s10526-0099234-9

Cocuzza, G.E.M., Alberto, U., Hernández-Suárez, E., Siverio, F., Di Silvestro, S., Tena, A., Rapisarda, C., 2017. A review on Trioza erytreae (African citrus psyllid), now in mainland Europe, and its potential risk as vector of huanglongbing (HLB) in citrus. J. Pest Sci. 90, 1-17. https://doi.org/10.1007/s10340-016-0804-1

Colomer, I., Aguado, P., Medina, P., Heredia, R.M., Fereres, A., Belda, J.E., Viñuela, E., 2011. Field trial measuring the compatibility of methoxyfenozide and flonicamid with Orius laevigatus Fieber (Hemiptera: Anthocoridae) and Amblyseius swirskii (AthiasHenriot) (Acari: Phytoseiidae) in a commercial pepper greenhouse. Pest Manag. Sci. 67, 1237-1244. https://doi.org/10.1002/ps.2173

Costanza, R., D’arge, R., De Groot, R., Farber, S., Grasso, M., Hannon, B., Limburg, K., Naeem, S., O’neill, R. V, Paruelo, J., Raskin, R.G., Sutton, P., van den Belt, M., 1998. The value of the world's ecosystem services and natural capital. Nature 387, 253-260.

Cowles, R.S., Montgomery, M.E., Cheah, C.A.S.J., 2006. Activity and residues of imidacloprid applied to soil and tree trunks to control hemlock woolly adelgid (Hemiptera: Adelgidae) in forests. J. Econ. Entomol. 99, 1258-1267. https://doi. org/10.1093/jee/99.4.1258

Cresswell, J.E., Robert, F.X.L., Florance, H., Smirnoff, N., 2014. Clearance of ingested neonicotinoid pesticide (imidacloprid) in honey bees (Apis mellifera) and bumblebees (Bombus terrestris). Pest Manag. Sci. 70, 332-337.

David, A., Botías, C., Abdul-Sada, A., Nicholls, E., Rotheray, E.L., Hill, M., Goulson, D., 2016. Widespread contamination of wildflower and bee-collected pollen. Environ. Int. $88,169-178$. 
de-Miguel, S., Pukkala, T., Yeşil, A., 2014. Integrating pine honeydew honey production into forest management optimization. Eur. J. For. Res. 133, 423-432. https://doi. org/10.1007/s10342-013-0774-2

DeBach, P., Rosen, D., 1974. Biological control by natural enemies. Cambridge Univ. Press. London

Dembilio, Ó., Riba, J.M., Gamón, M., Jacas, J.A., 2015. Mobility and efficacy of abamectin and imidacloprid against Rhynchophorus ferrugineus in Phoenix canariensis by different application methods. Pest Manag. Sci. 71, 1091-1098. https://doi.org/10.1002/ps.3889

Desneux, N., Barta, R.J., Hoelmer, K.A., Hopper, K.R., Heimpel, G.E., 2009. Multifaceted determinants of host specificity in an aphid parasitoid. Oecologia 160, 387-398. https://doi.org/10.1007/s00442-009-1289-x

Desneux, N., Decourtye, A., Delpuech, J.-M., 2007. The sublethal effects of pesticides on beneficial arthropods. Annu. Rev. Entomol. 52, 81-106. https://doi.org/10.1146/ annurev.ento.52.110405.091440

Devine, G.J., Harling, Z.K., Scarr, A.W., Devonshire, A.L., 1996. Lethal and sublethal effects of imidacloprid on nicotine-tolerant Myzus nicotianae and Myzus persicae. Pestic. Sci. 48, 57-62. https://doi.org/10.1002/(SICI)1096-9063(199609)48:1<57::AIDPS435 $>3.0 . C O ; 2-9$

DeVries, P.J., Baker, I., 1989. Butterfly exploitation of an ant-plant mutualism: adding insult to herbivory. J. N. Y. Entomol. Soc. 97, 332-340.

DiBartolomeis, M., Kegley, S., Mineau, P., Radford, R., Klein, K., 2019. An assessment of acute insecticide toxicity loading (AITL) of chemical pesticides used on agricultural land in the United States. PLoS One 14, e0220029. https://doi.org/10.1371/journal. pone.0220029

Dieckhoff, C., Theobald, J.C., Wäckers, F.L., Heimpel, G.E., 2014. Egg load dynamics and the risk of egg and time limitation experienced by an aphid parasitoid in the field. Ecol. Evol. 4, 1739-1750. https://doi.org/10.1002/ece3.1023

Dieckmann, Y., Ishaque, M., Muenster, I., Picard, L., Benz, A., Langewald, J., Kreuz, K., Koehle, H., Goerth, F.C., Raether, R.B., Montag, J., Huber-Moulliet, U., Kerl, W., 2010a. Systemicity enhancers. Patent No. US, 204045, A1.

Dieckmann, Y., Ishaque, M., Münster, I., Picard, L., Kerl, W., Langewald, J., Kreuz, K., Köhle, H., Christian, F., 2010b. Agrochemical Formulations Comprising Co-Polymers Based on Ethylenically Unsaturated Dicarboxylic Mono and Diesters. Patent No. US 2010/0063167, A1.

Dieckmann, Y., Ishaque, M., Münster, I., Picard, L., Kerl, W., Langewald, J., Kreuz, K., Köhle, H., Görth, F.C., 2010c. Agrochemical Formulations Comprising 1-vinyl-2pyrrolidinone Co-Polymers. Patent No. US 2010/0075849, A1 
Dirzo, R., Young, H.S., Galetti, M., Ceballos, G., Isaac, N.J.B., Collen, B., 2014. Defaunation in the Antrhopocene. Science. 345, 401-406. https://doi.org/10.1126/science.1251817

Dittrich, V., Hassan, S.O., Ernst, G.H., 1986. Development of a new primary pest of cotton in the Sudan: Bemisia tabaci, the whitefly. Agric. Ecosyst. Environ. 17, 137-142.

Dively, G.P., Kamel, A., 2012. Insecticide residues in pollen and nectar of a cucurbit crop and their potential exposure to pollinators. J. Agric. Food Chem. 60, 4449-4456. https://doi.org/10.1021/jf205393x

Donley, N., 2019. The USA lags behind other agricultural nations in banning harmful pesticides. Environ. Heal. A Glob. Access Sci. Source 18, 44. https://doi.org/10.1186/ s12940-019-0488-0

Dos Santos, F.C., Halinski, R., de Souza dos Santos, D.P., Almeida, E.A.B., Blochtein, B., 2019. Looking beyond the flowers: associations of stingless bees with sap-sucking insects. Sci. Nat. 106, 12. https://doi.org/https://doi.org/10.1007/s00114-019-1608-y

Douglas, M.R., Tooker, J.F., 2015. Large-scale deployment of seed treatments has driven rapid increase in use of neonicotinoid insecticides and preemptive pest management in U.S. Field crops. Environ. Sci. Technol. 49, 5088-5097. https://doi.org/10.1021/ es506141g

Downes, W.L., Dahlem, G.A., 1987. Keys to the evolution of Diptera: role of Homoptera. Environ. Entomol. 16, 847-854. https://doi.org/10.1093/ee/16.4.847

Easton, A.H., Goulson, D., 2013. The neonicotinoid insecticide imidacloprid repels pollinating flies and beetles at field-realistic concentrations. PLoS One 8, e54819. https://doi.org/10.1371/journal.pone.0054819

Eddleston, M., Buckley, N.A., Eyer, P., Dawson, A.H., 2008. Management of acute organophosphorus pesticide poisoning. Lancet 371, 597-607. https://doi.org/10.1016/ S0140-6736(07)61202-1

Ejaz, M., Ali Shad, S., 2017. Spirotetramat resistance selected in the Phenacoccus solenopsis (Homoptera: Pseudococcidae): cross-resistance patterns, stability, and fitness costs analysis. J. Econ. Entomol. 110, 1226-1234. https://doi.org/10.1093/jee/tox045

El-Zahi, E.Z.S., El-Salam Aref, S.A., Mohammad Korish, S.K., 2016. The cotton mealybug, Phenacoccus solenopsis Tinsley (Hemiptera: Pseudococcidae) as a new menace to cotton in Egypt and its chemical control. J. Plant Prot. Res. 56, 111-115. https://doi. org/10.1515/jppr-2016-0017

El Kady, H., Devine, G.J., 2003. Insecticide resistance in Egyptian populations of the cotton whitefly, Bemisia tabaci (Hemiptera: Aleyrodidae). Pest Manag. Sci. 59, 865871. https://doi.org/10.1002/ps.687

Elbert, A., Nauen, R., 2000. Resistance of Bemisia tabaci (Homoptera: Aleyrodidae) to insecticides in Southern Spain with special reference to neonicotinoids. Pest Manag. 
Sci. 56, 60-64. https://doi.org/10.1002/(SICI)1526-4998(200001)56:1<60::AIDPS88>3.0.CO;2-K

Elhag, E.A., Horn, D.J., 1983. Resistance of greenhouse whitefly (Homoptera: Aleyrodidae) to insecticides in selected Ohio greenhouses. J. Econ. Entomol. 76, 945-948. https:// doi.org/10.1093/jee/76.4.945

Environmental Protection Agency, 2021. Schedule for review of neonicotinoid pesticides. URL https://www.epa.gov/pollinator-protection/schedule-review-neonicotinoidpesticides

EPA, 2016. Addendum to the environmental fate and ecological risk assessment for sulfoxaflor registration. United States Environ. Prot. Agency.

Erdogan, C., Moores, G.D., Oktay Gurkan, M., Gorman, K.J., Denholm, I., 2008. Insecticide resistance and biotype status of populations of the tobacco whitefly Bemisia tabaci (Hemiptera: Aleyrodidae) from Turkey. Crop Prot. 27, 600-605. https://doi. org/10.1016/j.cropro.2007.09.002

European Commission, 2021. Neonicotinoids, emergency authorisations in the Member States. URL https://ec.europa.eu/food/plants/pesticides/approval-active-substances/ renewal-approval/neonicotinoids_en

European Commission, 2018. Implementing Commission Regulations 2018/783/EC, 2018/784/EC, 2018/785/EC. L 132, 30.5.2018. Official Journal of the European Union.

European Commission, 2020. Neonicotinoids, emergency authorisations in the Member States. URL https://ec.europa.eu/food/plant/pesticides/approval_active_substances/ approval_renewal/neonicotinoids_en

European Food Safety Authority, 2018. Evaluation of the data on clothianidin, imidacloprid and thiamethoxam for the updated risk assessment to bees for seed treatments and granules in the EU. EFSA J. 15, 1378E. https://doi.org/10.2903/sp.efsa.2018.EN-1378

European Food Safety Authority, 2014. Conclusion on the peer review of the pesticide risk assessment of the active substance sulfoxaflor. EFSA J. 12, 3692. https://doi. org/10.2903/j.efsa.2014.3692

European Food Safety Authority, 2013a. Conclusion on the peer review of the pesticide risk assessment for bees for the active substance imidacloprid. EFSA J. 11, 3068. https://doi.org/10.2903/j.efsa.2013.3068.

European Food Safety Authority, 2013b. Conclusion on the peer review of the pesticide risk assessment of the active substance spirotetramat. EFSA J. 11, 3243 . https://doi.org/10.2903/j.efsa.2013.3243

European Food Safety Authority, 2013c. Conclusion on the peer review of the pesticide risk assessment for bees for the active substance fipronil. EFSA J. 11, 3158. https://doi. org/10.2903/j.efsa.2013.3158 
Ewart, W.H., Metcalf, R.L., 1956. Preliminary studies of sugars and amino acids in the honeydews of five species of coccids feeding on citrus in California. Ann. Entomol. Soc. Am. 49, 441-447. https://doi.org/10.1093/aesa/49.5.441

Fand, B.B., Amala, U., Yadav, D.S., Rathi, G., Mhaske, S.H., Upadhyay, A., Ahammed Shabeer, T.P., Kumbhar, D.R., 2020. Bacterial volatiles from mealybug honeydew exhibit kairomonal activity toward solitary endoparasitoid Anagyrus dactylopii. J. Pest Sci. 93, 195-206. https://doi.org/10.1007/s10340-019-01150-4

Fenner, K., Canonica, S., Wackett, L., Elsner, M., 2013. Evaluating pesticide degradation in the environment: blind spots and emerging opportunities. Science 341, 752-758. https://doi.org/10.1126/science.1236281

Fernández, E., Grávalos, C., Haro, P.J., Cifuentes, D., Bielza, P., 2009. Insecticide resistance status of Bemisia tabaci Q-biotype in south-eastern Spain. Pest Manag. Sci. 65, 885891. https://doi.org/10.1002/ps.1769

Fernández, V., Khayet, M., Montero-Prado, P., Heredia-Guerrero, J., Liakopoulos, G., Karabourniotis, G., del Río, V., Domínguez, E., Tacchini, I., Nerín, C., Val, J., Heredia, A., 2011. New insights into the properties of pubescent surfaces: Peach fruit as a model. Plant Physiol. 156, 2098-2108. https://doi.org/10.1104/ pp.111.176305

Fischer, M.K., Shingleton, A.W., 2001. Host plant and ants influence the honeydew sugar composition of aphids. Funct. Ecol. 15, 544-550. https://doi.org/10.1046/j.02698463.2001.00550.x

Foley, J.A., Ramankutty, N., Brauman, K.A., Cassidy, E.S., Gerber, J.S., Johnston, M., Mueller, N.D., O’Connell, C., Ray, D.K., West, P.C., Balzer, C., Bennett, E.M., Carpenter, S.R., Hill, J., Monfreda, C., Polasky, S., Rockström, J., Sheehan, J., Siebert, S., Tilman, D., Zaks, D.P.M., 2011. Solutions for a cultivated planet. Nature 478, 337-342. https:// doi.org/10.1038/nature10452

Food and Agriculture Organization of the United Nations, 2017. The use of integrated measures in a systems approach for pest risk managmeent. ISPM 14

Food and Agriculture Organization of the United Nations, 2018. Requirements for the use of temperature treatments as phytosanitary measures. ISPM 42

FAOSTAT, 2021. Food and Agriculture Organization of the United Nations. URL http:// www.fao.org/faostat/en/\#data/QC

Foster, S.P., Cox, D., Oliphant, L., Mitchinson, S., Denholm, I., 2008. Correlated responses to neonicotinoid insecticides in clones of the peach-potato aphid, Myzus persicae (Hemiptera: Aphididae). Pest Manag. Sci. 64, 1111-1114. https://doi.org/10.1002/ps 
Foster, S.P., Denholm, I., Thompson, R., 2003. Variation in response to neonicotinoid insecticides in peach-potato aphids, Myzus persicae (Hemiptera: Aphididae). Pest Manag. Sci. 59, 166-173. https://doi.org/10.1002/ps.570

Franco, J.C., Silva, E.B., Cortegano, E., Campos, L., Branco, M., Zada, A., Mendel, Z., 2008. Kairomonal response of the parasitoid Anagyrus spec. nov. near pseudococci to the sex pheromone of the vine mealybug. Entomol. Exp. Appl. 126, 122-130. https:// doi.org/10.1111/j.1570-7458.2007.00643.x

Frank, S.D., Tooker, J.F., 2020. Neonicotinoids pose undocumented threats to food webs. Proc. Natl. Acad.Sci. U.S.A. 117, 22609-22613.https://doi.org/10.1073/pnas.2017221117

Fratoni, S., Duarte, M.V.A., Vangansbeke, D., Wäckers, F.L., Dicke, M., Pekas, A., 2019. A bittersweet meal: the impact of sugar solutions and honeydew on the fitness of two predatory gall midges. Biol. Control 140, 104098. https://doi.org/10.1016/J. BIOCONTROL.2019.104098

Frewin, A.J., Schaafsma, A.W., Hallett, R.H., 2014. Susceptibility of Aphelinus certus (Hymenoptera: Aphelinidae) to neonicotinoid seed treatments used for soybean pest management. J. Econ. Entomol. 107, 1450-1457. https://doi.org/10.1603/ec13523

Frewin, A.J., Xue, Y., Welsman, J.A., Broadbent, B.A., Schaafsma, A.W., Hallett, R.H., 2010. Development and parasitism by Aphelinus certus (Hymenoptera: Aphelinidae), a parasitoid of Aphis glycines (Hemiptera: Aphididae). Environ. Entomol. 39, 15701578. https://doi.org/10.1603/en09312

Gallai, N., Salles, J.M., Settele, J., Vaissière, B.E., 2009. Economic valuation of the vulnerability of world agriculture confronted with pollinator decline. Ecol. Econ. 68, 810-821. https://doi.org/10.1016/j.ecolecon.2008.06.014

Garibaldi, L.A., Steffan-dewenter, I., Winfree, R., Aizen, M.A., Bommarco, R., Cunningham, S.A., Kremen, C., Carvalheiro, L.G., Harder, L.D., Ohad, A., Bartomeus, I., Benjamin, F., Boreux, V., Cariveau, D., Chacoff, N.P., Dudenhöffer, J.H., Freitas, B.M., Ghazoul, J., Greenleaf, S., Hipólito, J., Holzschuh, A., Howlett, B., Isaacs, R., Javorek, S.K., Kennedy, C.M., Krewenka, K.M., Krishnan, S., Mandelik, Y., Mayfield, M.M., Motzke, I., Munyuli, T., Nault, B.A., Otieno, M., Petersen, J., Pisanty, G., Potts, S.G., Rader, R., Ricketts, T.H., Rundlöf, M., Seymour, C.L., Schüepp, C., Szentgyörgyi, H., Taki, H., Tscharntke, T., Vergara, C.H., Viana, B.F., Wanger, T.C., Westphal, C., Williams, N., Klein, A.M., 2013. Wild Pollinators Enhance Fruit Set of Crops Regardless of Honey Bee Abundance. Science 339, 1608-1611.

Gierer, F., Vaughan, S., Slater, M., Thompson, H.M., Elmore, J.S., Girling, R.D., 2019. A review of the factors that influence pesticide residues in pollen and nectar: Future research requirements for optimising the estimation of pollinator exposure. Environ. Pollut. 249, 236-247. https://doi.org/10.1016/j.envpol.2019.03.025 
GIP Citricos, 2021. Instituto Valenciano de Investigaciones Agrarias. URL http:// gipcitricos.ivia.es/area/plagas-principales/diaspididos/piojo_rojo_california.

Girolami, V., Marzaro, M., Vivan, L., Mazzon, L., Greatti, M., Giorio, C., Marton, D., Tapparo, A., 2012. Fatal powdering of bees in flight with particulates of neonicotinoids seed coating and humidity implication. J. Appl. Entomol. 136, 17-26. https://doi. org/10.1111/j.1439-0418.2011.01648.x

Girolami, V., Mazzon, L., Squartini, A., Mori, N., Marzaro, M., Di bernardo, A., Greatti, M., Giorio, C., Tapparo, A., 2009. Translocation of neonicotinoid insecticides from coated seeds to seedling guttation drops: a novel way of intoxication for bees. J. Econ. Entomol. 102, 1808-1815. https://doi.org/10.1603/029.102.0511

Goatley, J.L., Lewis, R.W., 1966. Composition of guttation fluid from rye, wheat, and barley seedlings. Plant Physiol. 41, 373-375. https://doi.org/10.1104/pp.41.3.373

Gols, R., WallisDeVries, M.F., van Loon, J.J.A., 2020. Reprotoxic effects of the systemic insecticide fipronil on the butterfly Pieris brassicae. Proc. R. Soc. B Biol. Sci. 287, 20192665. https://doi.org/10.1098/rspb.2019.2665

Gómez, J.A., Campos, M., Guzmán, G., Castillo-Llanque, F., Vanwalleghem, T., Lora, Á., Giráldez, J. V., 2018. Soil erosion control, plant diversity, and arthropod communities under heterogeneous cover crops in an olive orchard. Environ. Sci. Pollut. Res. 25, 977-989. https://doi.org/10.1007/s11356-016-8339-9

Gontijo, P.C., Moscardini, V.F., Michaud, J., Carvalho, G.A., 2015. Non-target effects of two sunflower seed treatments on Orius insidiosus (Hemiptera: Anthocoridae). Pest Manag. Sci. 71, 515-522. https://doi.org/10.1002/ps.3798

Gorman, K., Slater, R., Blande, J.D., Clarke, A., Wren, J., McCaffery, A., Denholm, I., 2010. Cross-resistance relationships between neonicotinoids and pymetrozine in Bemisia tabaci (Hemiptera: Aleyrodidae). Pest Manag. Sci. 66, 1186-1190. https:// doi.org/10.1002/ps.1989

Goulson, D., 2013. An overview of the environmental risks posed by neonicotinoid insecticides. J. Appl. Ecol. 50, 977-987. https://doi.org/10.1111/1365-2664.12111

Goulson, D., Lye, G.C., Darvill, B., 2008. Decline and conservation of bumblebees. Annu. Rev. Entomol. 53, 191-208.

Government UK, 2021. Statement on the decision to issue - with strict conditions - emergency authorisation to use a product containing a neonicotinoid to treat sugar beet seed in 2021. URL https://www.gov.uk/government/publications/ neonicotinoid-product-as-seed-treatment-for-sugar-beet-emergency-authorisationapplication/statement-on-the-decision-to-issue-with-strict-conditions-emergencyauthorisation-to-use-a-product-containing-a-neonicoti 
Grafton-Cardwell, E.E., 1996. UC IPM pest management guidelines: citrus. University of California, DANR/Communications Services. URL http://ipm.ucanr.edu/PMG/ selectnewpest.citrus.html.

Grafton-Cardwell, E.E., Leigh, T.F., Bentley, W.J., Goodell, P.B., 1992. Cotton aphids have become resistant to commonly used pesticides. Calif. Agric. 46, 4-7.

Grafton-Cardwell, E.E., Stelinski, L.L., Stansly, P.A., 2013. Biology and management of Asian citrus psyllid, vector of the huanglongbing pathogens. Annu. Rev. Entomol. 58, 413-432. https://doi.org/10.1146/annurev-ento-120811-153542

Greatti, M., Barbattini, R., Stravisi, A., Sabatini, A.G., Rossi, S., 2006. Presence of the a.i. imidacloprid on vegetation near corn fields sown with Gaucho ${ }^{\circledR}$ dressed seeds. Bull. Insectology 59, 99-103.

Grunwald, I., Rupprecht, I., Schuster, G., Kloppstech, K., 2003. Identification of guttation fluid proteins: The presence of pathogenesis-related proteins in non-infected barley plants. Physiol. Plant. 119, 192-202. https://doi.org/10.1034/j.1399-3054.2003.00202.x

Guedes, R.N.C., Smagghe, G., Stark, J.D., Desneux, N., 2016. Pesticide-induced stress in arthropod pests for optimized integrated pest management programs. Annu. Rev. Entomol. 61, 43-62. https://doi.org/10.1146/annurev-ento-010715-023646

Gunstone, T., Cornelisse, T., Klein, K., Dubey, A., Donley, N., 2021. Pesticides and soil invertebrates: a hazard assessment. Front. Environ. Sci. 9, 1-21. https://doi. org/10.3389/fenvs.2021.643847

Gupta, R.C., Miller Mukherjee, I.R., Doss, R.B., Malik, J.K., Milatovic, D., 2017. Organophosphates and carbamates. Reprod. Dev. Toxicol. 609-631. https://doi. org/10.1016/B978-0-12-804239-7.00035-4

Gurr, G.M., Wratten, S.D., Landis, D.A., You, M., 2017. Habitat management to suppress pest populations: progress and prospects. Annu. Rev. Entomol. 62, 91-109.

Hagen, K.S., 1962. Biology and ecology of predaceous Coccinellidae. Annu. Rev. Entomol. 7, 289-326. https://doi.org/10.1146/annurev.en.07.010162.001445

Hagenbucher, S., Wäckers, F.L., Romeis, J., 2014. Aphid honeydew quality as a food source for parasitoids is maintained in Bt cotton. PLoS One 9, e107806. https://doi. org/10.1371/journal.pone.0107806

Hallmann, C.A., Foppen, R.P.B., Van Turnhout, C.A.M., De Kroon, H., Jongejans, E., 2014. Declines in insectivorous birds are associated with high neonicotinoid concentrations. Nature 511, 341. https://doi.org/10.1038/nature13531

Hallmann, C.A., Sorg, M., Jongejans, E., Siepel, H., Hofland, N., Schwan, H., Stenmans, W., Müller, A., Sumser, H., Hörren, T., Goulson, D., De Kroon, H., 2017. More than 75 percent decline over 27 years in total flying insect biomass in protected areas. PLoS One 12, e0185809. https://doi.org/10.1371/journal.pone.0185809 
Hallmann, C.A., Ssymank, A., Sorg, M., de Kroon, H., Jongejans, E., 2021. Insect biomass decline scaled to species diversity: General patterns derived from a hoverfly community. Proc. Natl. Acad. Sci. U. S. A. 118, e2002554117. https://doi.org/10.1073/ PNAS.2002554117

Harrewijn, P., Kayser, H., 1997. Pymetrozine, a fast-acting and selective inhibitor of aphid feeding. In-situ studies with electronic monitoring of feeding behaviour. Pestic. Sci. 49, 130140. https://doi.org/10.1002/(SICI)1096-9063(199702)49:2<130::AID-PS509>3.0.CO;2-U

Harvey, J.A., Heinen, R., Armbrecht, I., Basset, Y., Baxter-Gilbert, J.H., Bezemer, T.M., Böhm, M., Bommarco, R., Borges, P.A.V., Cardoso, P., Clausnitzer, V., Cornelisse, T., Crone, E.E., Dicke, M., Dijkstra, K.D.B., Dyer, L., Ellers, J., Fartmann, T., Forister, M.L., Furlong, M.J., Garcia-Aguayo, A., Gerlach, J., Gols, R., Goulson, D., Habel, J.C., Haddad, N.M., Hallmann, C.A., Henriques, S., Herberstein, M.E., Hochkirch, A., Hughes, A.C., Jepsen, S., Jones, T.H., Kaydan, B.M., Kleijn, D., Klein, A.M., Latty, T., Leather, S.R., Lewis, S.M., Lister, B.C., Losey, J.E., Lowe, E.C., Macadam, C.R., Montoya-Lerma, J., Nagano, C.D., Ogan, S., Orr, M.C., Painting, C.J., Pham, T.H., Potts, S.G., Rauf, A., Roslin, T.L., Samways, M.J., Sanchez-Bayo, F., Sar, S.A., Schultz, C.B., Soares, A.O., Thancharoen, A., Tscharntke, T., Tylianakis, J.M., Umbers, K.D.L., Vet, L.E.M., Visser, M.E., Vujic, A., Wagner, D.L., WallisDeVries, M.F., Westphal, C., White, T.E., Wilkins, V.L., Williams, P.H., Wyckhuys, K.A.G., Zhu, Z.R., de Kroon, H., 2020. International scientists formulate a roadmap for insect conservation and recovery. Nat. Ecol. Evol. 4, 174-176. https://doi.org/10.1038/s41559-019-1079-8

He, Y.-X., Weng, Q.-Y., Huang, J., Liang, Z.-S., Lin, G.-J., Wu, D.-D., 2007. Insecticide resistance of Bemisia tabaci field populations. Chinese J. Appl. Ecol. 18, 1578-1582.

Heil, M., 2015. Extrafloral nectar at the plant-insect interface: a spotlight on chemical ecology, phenotypic plasticity, and food webs. Annu. Rev. Entomol. 60, 213-232. https://doi.org/10.1146/annurev-ento-010814-020753

Heimpel, G.E., Jervis, M., 2005. Does floral nectar improve biological control by parasitoids?, in: Wackers, F.L., van Rijn, P.C.J., Bruin, J. Plant-provided food for carnivorous insects: a protective mutualism and its applications. Cambridge University Press, Cambridge, UK. pp. 267-304. https://doi.org/10.1017/CBO9780511542220.010

Heimpel, G.E., Jervis, M.A., 2005. Phytophagy, in: Insects as natural enemies. Springer, The Netherlands. https://doi.org/doi.org/10.1007/978-1-4020-2625-6_8

Heimpel, G.E., Lee, J.C., Wu, Z., Weiser, L., Wäckers, F., Jervis, M.A., 2004. Gut sugar analysis in field-caught parasitoids: Adapting methods originally developed for biting flies. Int. J. Pest Manag. 50, 193-198. https://doi.org/10.1080/09670870410001731925 Heimpel, G.E., Lundgren, J.G., 2000. Sex ratios of commercially reared biological control agents. Biol. Control 19, 77-93. https://doi.org/10.1006/bcon.2000.0849 
Heimpel, G.E., Mills, N.J., 2017. Biological control: ecology and applications. Cambridge University Press. Cambridge

Heimpel, G.E., Yang, Y., Hill, J.D., Ragsdale, D.W., 2013. Environmental consequences of invasive species: greenhouse gas emissions of insecticide use and the role of biological control in reducing emissions. PLoS One 8, e72293. https://doi.org/10.1371/journal. pone. 0072293

Henry, M., Béguin, M., Requier, F., Rollin, O., Odoux, J.F., Aupinel, P., Aptel, J., Tchamitchian, S., Decourtye, A., 2012. A common pesticide decreases foraging success and survival in honey bees. Science 336, 348-350. https://doi.org/10.1126/ science. 1215039

Herren, H.R., Neuenschwander, P., 1991. Biological control of cassava pests in Africa. Annu. Rev. Entomol. 36, 257-283. https://doi.org/10.1146/annurev.en.36.010191.001353

Herrera, C.M., 1990. Bumble bees feeding on non-plant food sources. Bee World 71, 6769. https://doi.org/10.1080/0005772X.1990.11099039

Herron, G.A., Gibson, T.S., Horwood, M.A., 1993. Insecticide resistance in Myzus persicae (Sulzer) (Hemiptera: Aphididae) in Southeastern Australia. Aust. J. Entomol. 32, 23-27. https://doi.org/10.1111/j.1440-6055.1993.tb00537.x

Herron, G.A., Rophail, J., 1994. Insecticide resistance detected in Myzus persicae (Sulzer) (Hemiptera: Aphididae) from New South Wales cotton. Aust. J. Entomol. 33, 263264. https://doi.org/10.1111/j.1440-6055.1994.tb01227.x

Herron, G.A., Wilson, L.J., 2011. Neonicotinoid resistance in Aphis gossypii Glover (Aphididae: Hemiptera) from Australian cotton. Aust. J. Entomol. 50, 93-98. https:// doi.org/10.1111/j.1440-6055.2010.00788.x

Hilbeck, A., Meier, M., Römbke, J., Jänsch, S., Teichmann, H., Tappeser, B., 2011. Environmental risk assessment of genetically modified plants - concepts and controversies. Environ. Sci. Eur. 23, 1-12. https://doi.org/10.2478/ffp-2014-0011

Hladik, M.L., Kolpin, D.W., Kuivila, K.M., 2014. Widespread occurrence of neonicotinoid insecticides in streams in a high corn and soybean producing region, USA. Environ. Pollut. 193, 189-196. https://doi.org/10.1016/j.envpol.2014.06.033

Hogervorst, P.A.M., Wäckers, F.L., Carette, A.C., Romeis, J., 2008. The importance of honeydew as food for larvae of Chrysoperla carnea in the presence of aphids. J. Appl. Entomol. 132, 18-25. https://doi.org/10.1111/j.1439-0418.2007.01247.x

Hogervorst, P.A.M., Wäckers, F.L., Romeis, J., 2007. Detecting nutritional state and food source use in field-collected insects that synthesize honeydew oligosaccharides. Funct. Ecol. 21, 936-946. https://doi.org/10.1111/j.1365-2435.2007.01297.x

Holland, J.M., Bianchi, F.J., Entling, M.H., Moonen, A.C., Smith, B.M., Jeanneret, P., 2016. Structure, function and management of semi-natural habitats for conservation 
biological control: a review of European studies. Pest Manag. Sci. 72, 1638-1651. https://doi.org/10.1002/ps.4318

Hölldobler, B., Wilson, E.O., 1990. The Ants, Harvard University Press. https://doi. org/10.1002/ejoc.201200111

Holman, J., 2009. Host plant catalog of aphids, Springer Science \& Business Media. https://doi.org/10.1007/978-1-4020-8286-3

Hopper, K.R., Lanier, K., Rhoades, J.H., Hoelmer, K.A., Meikle, W.G., Heimpel, G.E., O’Neil, R.J., Voegtlin, D.G., Woolley, J.B., 2017. Host specificity of Aphelinus species collected from soybean aphid in Asia. Biol. Control 115, 55-73. https://doi. org/10.1016/j.biocontrol.2017.09.004

Humann-Guilleminot, S., Binkowski, Ł.J., Jenni, L., Hilke, G., Glauser, G., Helfenstein, F., 2019. A nation-wide survey of neonicotinoid insecticides in agricultural land with implications for agri-environment schemes. J. Appl. Ecol. 56, 1502-1514. https://doi. org/10.1111/1365-2664.13392

Hurej, M., Peters, D., 1989. Sublethal effects of aldicarb on the behaviour of Aphis fabae and two clones of Myzus persicae and on the transmission of beet mosaic virus by these aphids. Entomol. Exp. Appl. 81-86. https://doi.org/10.1111/j.1570-7458.1989.tb02318.x

Hurley, T., Mitchell, P., 2017. Value of neonicotinoid seed treatments to US soybean farmers. Pest Manag. Sci. 73, 102-112. https://doi.org/10.1002/ps.4424

Ide, T., Suzuki, N., Katayama, N., 2007. The use of honeydew in foraging for aphids by larvae of the ladybird beetle, Coccinella septempunctata L. (Coleoptera: Coccinellidae). Ecol. Entomol. 32, 455-460. https://doi.org/10.1111/j.1365-2311.2007.00896.x

Insecticide Resistance Action Committee, 2020. Modes of Action (MoA) Classification | IRAC. URL https://www.irac-online.org/modes-of-action/.

International Rice Research Institute, 1984. Present and future directions of chemical control research. In: Proceeding of the FAO/IRRI Workshop on Judicious and Efficient Use of Insecticides on Rice. pp. 80-81. https://doi.org/10.1177/003072708501400322 Jansen, J.P., 2000. A three-year field study on the short-term effects of insecticides used to control cereal aphids on plant-dwelling aphid predators in winter wheat. Pest Manag. Sci. 56, 533-539. https://doi.org/10.1002/(SICI)1526-4998(200006)56:6<533::AIDPS165>3.0.CO;2-S

Jansen, J.P., Defrance, T., Warnier, A.M., 2011. Side effects of flonicamide and pymetrozine on five aphid natural enemy species. BioControl 56, 759-770. https://doi.org/10.1007/ s10526-011-9342-1

Jervis, M., 2005. Insects as natural enemies : a practical perspective. Springer. The Netherlands 
Jeschke, P., Nauen, R., Schindler, M., Elbert, A., 2011. Overview of the status and global strategy for neonicotinoids. J. Agric. Food Chem. 59, 2897-2908. https://doi. org/10.1021/jf101303g

Jiang, J., Ma, D., Zou, N., Yu, X., Zhang, Z., Liu, F., Mu, W., 2018. Concentrations of imidacloprid and thiamethoxam in pollen, nectar and leaves from seed-dressed cotton crops and their potential risk to honeybees (Apis mellifera L.), Chemosphere 201, 159-167. https://doi.org/10.1016/j.chemosphere.2018.02.168

Jones, A.G., Hoover, K., Pearsons, K., Tooker, J.F., Felton, G.W., 2020. Potential impacts of translocation of neonicotinoid insecticides to cotton (Gossypium hirsutum (Malvales: Malvaceae)) extrafloral nectar on parasitoids. Environ. Entmol. 49, 159-168. https:// doi.org/10.1093/ee/nvz157

Joseph, J.R., Ameline, A., Couty, A., 2011. Effects on the aphid parasitoid Aphidius ervi of an insecticide (Plenum ${ }^{\oplus}$, pymetrozine) specific to plant-sucking insects. Phytoparasitica 39, 35-41. https://doi.org/10.1007/s12600-010-0134-4

Kaiser, M.E., Noma, T., Brewer, M.J., Pike, K.S., Vockeroth, J.R., Gaimari, S.D., 2007. Ecology and population biology: Hymenopteran parasitoids and Dipteran predators found using soybean aphid after its midwestern United States invasion. Ann. Entomol. Soc. Am. 100, 196-205. https://doi.org/10.1603/0013-8746(2007)100[196:HPADPF]2.0.CO;2

Kampfraath, A.A., Giesen, D., van Gestel, C.A.M., Le Lann, C., 2017. Pesticide stress on plants negatively affects parasitoid fitness through a bypass of their phytophage hosts. Ecotoxicology 26, 383-395. https://doi.org/10.1007/s10646-017-1771-x

Kang, C.Y., Wu, G., Miyata, T., 2006. Synergism of enzyme inhibitors and mechanisms of insecticide resistance in Bemisia tabaci (Gennadius) (Hom., Aleyrodidae). J. Appl. Entomol. 130, 377-385. https://doi.org/10.1111/j.1439-0418.2006.01075.x

Kaser, J.M., Heimpel, G.E., 2018. Impact of the parasitoid Aphelinus certus on soybean aphid populations. Biol. Control 127, 17-24. https://doi.org/10.1016/j. biocontrol.2018.08.014

Keeler, K.H., 1977. The extrafloral nectaries of Ipomoea carnea (Convolvulaceae). Am. J. Bot. 64, 1182. https://doi.org/10.2307/2442480

Kehoe, R., Frago, E., Sanders, D., 2020. Cascading extinctions as a hidden driver of insect decline. Ecol. Entomol. https://doi.org/10.1111/een.12985

Kessler, S.C., Tiedeken, E.J., Simcock, K.L., Derveau, S., Mitchell, J., Softley, S., Stout, J.C., Wright, G.A., 2015. Bees prefer foods containing neonicotinoid pesticides. Nature 521, 74-76. https://doi.org/10.1038/nature14414

Kilin, D., Nagata, T., Masuda, T., 1981. Development of carbamate resistance in the brown planthopper Nilaparvata lugens Stal (Homoptera: Delphacidae). Chem. Pharm. Bull. $16,1-6$. 
Klein, A.M., Vaissière, B.E., Cane, J.H., Steffan-Dewenter, I., Cunningham, S.A., Kremen, C., Tscharntke, T., 2007. Importance of pollinators in changing landscapes for world crops. Proc. R. Soc. B Biol. Sci. 274, 303-313. https://doi.org/10.1098/rspb.2006.3721

Knodel, J.J., Shrestha, G., 2018. Pulse crops: pest management of wireworms and cutworms in the northern great plains of United States and Canada. Ann. Entomol. Soc. Am. 111, 195-204. https://doi.org/10.1093/aesa/say018

Komarnytsky, S., Borisjuk, N. V., Borisjuk, L.G., Alam, M.Z., Raskin, I., 2000. Production of recombinant proteins in tobacco guttation fluid. Plant Physiol. 124, 927-933. https://doi.org/10.1104/pp.124.3.927

Konrad, R., Wäckers, F.L., Romeis, J., Babendreier, D., 2009. Honeydew feeding in the solitary bee Osmia bicornis as affected by aphid species and nectar availability. J. Insect Physiol. 55, 1158-1166. https://doi.org/10.1016/j.jinsphys.2009.08.012

Krischik, V., Landmark, A.L., Heimpel, G.E., 2007. Soil-applied imidacloprid is translocated to nectar and kills nectar-feeding Anagyrus pseudococci (Girault) (Hymenoptera: Encyrtidae). Environ. Entomol. 36, 1238-45. https://doi. org/10.1603/0046-225X(2007)36

Krupke, C., Alford, A.M., Cullen, E.M., Hodgson, E.W., Knodel, J.J., McCornack, B., Potter, B.D., Spigler, M.I., Tilmon, K., Welch, K., 2017. Assessing the value and pest management window provided by neonicotinoid seed treatments for management of soybean aphid (Aphis glycines Matsumura) in the Upper Midwestern United States. Pest Manag. Sci. 73, 2184-2193. https://doi.org/10.1002/ps.4602

Krupke, C.H., Hunt, G.J., Eitzer, B.D., Andino, G., Given, K., 2012. Multiple routes of pesticide exposure for honey bees living near agricultural fields. PLoS One 7, e29268. https://doi.org/10.1371/journal.pone.0029268

Krupke, C.H., Tooker, J.F., 2020. Beyond the headlines: the influence of insurance pest management on an unseen, silent entomological majority. Front. Sustain. Food Syst. 4, 1-11. https://doi.org/10.3389/fsufs.2020.595855

Kyriakopoulou, K., Kandris, I., Pachiti, I., Kasiotis, K.M., Spyropoulou, A., Santourian, A., Kitromilidou, S., Pappa, G., Glossioti, M., 2017. Collection and analysis of pesticide residue data for pollen and nectar - Final Report. EFSA Support. Publ. 14. https://doi. org/10.2903/sp.efsa.2017.en-1303

Labrie, G., Gagnon, A.E., Vanasse, A., Latraverse, A., Tremblay, G., 2020. Impacts of neonicotinoid seed treatments on soil-dwelling pest populations and agronomic parameters in corn and soybean in Quebec (Canada). PLoS One 15, e0229136. https:// doi.org/10.1371/journal.pone.0229136 
Landis, D.A., Wratten, S.D., Gurr, G.M., 2000. Habitat management to conserve natural enemies of arthropod pests in agriculture. Annu. Rev. Entomol. 45, 175-201. https:// doi.org/10.1146/annurev.ento.45.1.175

Leach, H., Biddinger, D.J., Krawczyk, G., Smyers, E., Urban, J.M., 2019. Evaluation of insecticides for control of the spotted lanternfly, Lycorma delicatula, (Hemiptera: Fulgoridae), a new pest of fruit in the Northeastern U.S. Crop Prot. 124. https://doi. org/10.1016/j.cropro.2019.05.027

Lee, J.C., Andow, D.A., Heimpel, G.E., 2006. Influence of floral resources on sugar feeding and nutrient dynamics of a parasitoid in the field. Ecol. Entomol. 31, 470-480. https:// doi.org/10.1111/j.1365-2311.2006.00800.x

Lei, H., Tjallingii, W.F., Lenteren, J.C., 1997. Effect of tethering during EPG recorded probing by adults of the greenhouse whitefly. J. Appl. Entomol. 121, 211-217. https:// doi.org/10.1111/j.1439-0418.1997.tb01395.x

Leroy, P.D., Sabri, A., Heuskin, S., Thonart, P., Lognay, G., Verheggen, F.J., Francis, F., Brostaux, Y., Felton, G.W., Haubruge, E., 2011. Microorganisms from aphid honeydew attract and enhance the efficacy of natural enemies. Nat. Commun. 2. 348. https://doi. org/10.1038/ncomms 1347

Li, Y., Miao, R., Khanna, M., 2020. Neonicotinoids and decline in bird biodiversity in the United States. Nat. Sustain. 3. 1027-1035 https://doi.org/10.1038/s41893-020-0582-x Liao, X., Jin, R., Zhang, X., Ali, E., Mao, K., Xu, P., Li, J., Wan, H., 2018. Characterization of sulfoxaflor resistance in the brown planthopper, Nilaparvata lugens (Stål). Pest Manag. Sci. 75, 1646-1654. https://doi.org/10.1002/ps.5282

Ling, S., Zhang, J., HU, L., Zhang, R., 2009. Effect of fipronil on the reproduction, feeding, and relative fitness of brown planthopper, Nilaparvata lugens. Appl. Entomol. Zool. 44, 543-548. https://doi.org/10.1303/aez.2009.543

Lister, B.C., Garcia, A., 2018. Climate-driven declines in arthropod abundance restructure a rainforest food web. Proc. Nat. Acad. Sci. 201722477. https://doi.org/10.1073/ pnas. 1722477115

Liu, X., Zhu, Y., Dong, F., Xu, J., Zheng, Y., 2014. Dissipation and residue of flonicamid in cucumber, apple and soil under field conditions. Int. J. Environ. Anal. Chem. 94, 652-660. https://doi.org/10.1080/03067319.2013.871714

Losey, J.E., Vaughan, M., 2006. The Economic Value of Ecological Services Provided by Insects. Bioscience 56, 311-323.

Loukola, O.J., Perry, C.J., Coscos, L., Chittka, L., 2017. Bumblebees show cognitive flexibility by improving on an observed complex behavior. Science 355, 833-836. https://doi.org/10.1126/science.aag2360 
Lundgren, J.G., 2009. Relationships of Natural Enemies and Non-Prey Foods, Springer Science \& Business Media. Springer Science \& Business Media. https://doi. org/10.1007/978-1-4020-9235-0

Luquet, M., Peñalver-Cruz, A., Satour, P., Anton, S., Cortesero, A.M., Lavandero, B., Jaloux, B., 2021. Aphid honeydew may be the predominant sugar source for Aphidius parasitoids even in nectar-providing intercrops. Biol. Control 158. 104596. https:// doi.org/10.1016/j.biocontrol.2021.104596

Ma, K., Tang, Q., Zhang, B., Liang, P., Wang, B., Gao, X., 2019. Overexpression of multiple cytochrome $\mathrm{P} 450$ genes associated with sulfoxaflor resistance in Aphis gossypii Glover. Pestic. Biochem. Physiol. 157, 204-210. https://doi.org/10.1016/j.pestbp.2019.03.021

Magalhaes, L.C., Hunt, T.E., Siegfried, B.D., 2009. Efficacy of neonicotinoid seed treatments to reduce soybean aphid populations under field and controlled conditions in Nebraska. J. Econ. Entomol. 102, 187-195. https://doi.org/10.1603/029.102.0127

Manjon, C., Troczka, B.J., Zaworra, M., Beadle, K., Randall, E., Hertlein, G., Singh, K.S., Zimmer, C.T., Homem, R.A., Lueke, B., Reid, R., Kor, L., Kohler, M., Benting, J., Williamson, M.S., Davies, T.G.E., Field, L.M., Bass, C., Nauen, R., 2018. Unravelling the molecular determinants of bee sensitivity to neonicotinoid insecticides. Curr. Biol. 28, 1137-1143.e5. https://doi.org/10.1016/j.cub.2018.02.045

Marcić, D., Kljajić, P., Krnjajić, S., Perić, I., 2007. Studies of the efficacy of insecticides against pepper-infesting aphids (Aphididae). Acta Hortic. 729, 483-487. https://doi. org/10.17660/ActaHortic.2007.729.82

Martinou, A.F., Seraphides, N., Stavrinides, M.C., 2014. Lethal and behavioral effects of pesticides on the insect predator Macrolophus pygmaeus. Chemosphere 96, 167-173. https://doi.org/10.1016/j.chemosphere.2013.10.024

Masiá, A., Campo, J., Vázquez-Roig, P., Blasco, C., Picó, Y., 2013. Screening of currently used pesticides in water, sediments and biota of the Guadalquivir River Basin (Spain). J. Hazard. Mater. 263, 95-104. https://doi.org/10.1016/j.jhazmat.2013.09.035

Matsuda, K., Ihara, M., Sattelle, D.B., 2020. Neonicotinoid insecticides: molecular targets, resistance, and toxicity. Annu. Rev. Pharmacol. Toxicol. 60, 241-255. https://doi. org/10.1146/annurev-pharmtox-010818-021747

McClanahan, R.J., Founk, J., 1983. Toxicity of insecticides to the green peach aphid (Homoptera: Aphididae) in laboratory and field tests. J. Econ. Entomol. 76, 899-905. https://doi.org/10.1093/jee/76.4.899

Mccornack, B.P., Ragsdale, D.W., 2006. Efficacy of thiamethoxam to suppress soybean aphid populations in Minnesota soybean crop management. Crop Manag. 5, 1-8. https://doi.org/10.1094/CM-2006-0915-01-RS.Abstract 
Meiners, J.M., Griswold, T.L., Harris, D.J., Ernest, S.K.M., 2017. Bees without flowers: Before peak bloom, diverse native bees find insect-produced honeydew sugars. Am. Nat. 190, 281-291. https://doi.org/10.1086/692437

Michaud, J.P., Qureshi, J.A., 2006. Reproductive diapause in Hippodamia convergens (Coleoptera: Coccinellidae) and its life history consequences. Biol. Control 39, 193200. https://doi.org/10.1016/j.biocontrol.2006.04.004

Miksanek, J.R., 2020. Population ecology of Aphelinus certus, an adventive parasitoid of soybean aphid in North America, with implications for biological control. PhD thesis at University of Minnesota. Saint Paul, U.S.A.

Miksanek, J.R., Heimpel, G.E., 2019. A matrix model describing host-parasitoid population dynamics: The case of Aphelinus certus and soybean aphid. PLoS One 14, e0218217. https://doi.org/10.1371/journal.pone.0218217

Moens, J., de Clercq, P., Tirry, L., 2011. Side effects of pesticides on the larvae of the hoverfly Episyrphus balteatus in the laboratory. Phytoparasitica 39, 1-9. https://doi. org/10.1007/s 12600-010-0127-3

Mommaerts, V., Smagghe, G., 2011. Side-effects of pesticides on the pollinator bombus: an overview. In: Pesticides of the modern World. InTech, Rijeka, pp 507-552. https:// doi.org/10.5772/25254

Monkiedje, A., Spiteller, M., Maniepi, S.J.N., Sukul, P., 2007. Influence of metalaxyl- and mefenoxam-based fungicides on chemical and biochemical attributes of soil quality under field conditions in a southern humid forest zone of Cameroon. Soil Biol. Biochem. 39, 836-842. https://doi.org/10.1016/j.soilbio.2006.10.002

Monzo, C., Stansly, P.A., 2017. Economic injury levels for Asian citrus psyllid control in process oranges from mature trees with high incidence of huanglongbing. PLoS One 12, e0175333. https://doi.org/10.1371/journal.pone.0175333

Moores, G.D., Gao, X., Denholm, I., Devonshire, A.L., 1996. Characterisation of insensitive acetylcholinesterase in insecticide-resistant cotton aphids, Aphis gossypii Glover (Homoptera: Aphididae). Pestic. Biochem. Physiol. 56, 102-110. https://doi. org/10.1006/pest.1996.0064

Moreno-Ramírez, N., 2020. Honeydew: The hidden and sweet driver of direct and indirect interactions among insects. MSc thesis, Wageningen University, Wageningen, The Netherlands.

Morita, M., Ueda, T., Yoneda, T., Koyanagi, T., Haga, T., 2007. Flonicamid, a novel insecticide with a rapid inhibitory effect on aphid feeding. Pest Manag. Sci. 63, 969973. https://doi.org/10.1002/ps.1423 
Moser, S.E., Obrycki, J.J., 2009. Non-target effects of neonicotinoid seed treatments; mortality of coccinellid larvae related to zoophytophagy. Biol. Control 51, 487-492. https://doi.org/10.1016/j.biocontrol.2009.09.001

Mourtzinis, S., Krupke, C.H., Esker, P.D., Varenhorst, A., Arneson, N.J., Bradley, C.A., Byrne, A.M., Chilvers, M.I., Giesler, L.J., Herbert, A., Kandel, Y.R., Kazula, M.J., Hunt, C., Lindsey, L.E., Malone, S., Mueller, D.S., Naeve, S., Nafziger, E., Reisig, D.D., Ross, W.J., Rossman, D.R., Taylor, S., Conley, S.P., 2019. Neonicotinoid seed treatments of soybean provide negligible benefits to US farmers. Sci. Rep. 9, 11207. https://doi. org/10.1038/s41598-019-47442-8

Nagrare, V S, Kranthi, S., Kranthi, K.R., Naik, V.C.B., Deshmukh, V., 2016. Relative toxicity of insecticides against cotton mealybug Phenacoccus solenopsis Tinsley ( Hemiptera : Pseudococcidae ) and its fortuous parasitod Aenasius bambawalei Hayat ( Hymenoptera : Encyrtidae ). J. Appl. Nat. Sci. 8, 987-994.

Nagrare, V. S., Kranthi, S., Kranthi, K.R., Naik, V.C.B., Deshmukh, V., Naikwadi, B., Dahekar, A., 2016. Relative toxicity of insecticides against cotton mealybug Phenacoccus solenopsis Tinsley (Hemiptera:Pseudococcidae) and its fortuous parasitod Aenasius bambawalei Hayat (Hymenoptera: Encyrtidae). J. Appl. Nat. Sci. 8, 987-994. https://doi.org/10.31018/jans.v8i2.909

Natale, G.S., Vera-Candioti, J., Ruiz de Arcaute, C., Soloneski, S., Larramendy, M.L., Ronco, A.E., 2018. Lethal and sublethal effects of the pirimicarb-based formulation Aficida $^{\oplus}$ on Boana pulchella (Duméril and Bibron, 1841) tadpoles (Anura, Hylidae). Ecotoxicol. Environ. Saf. 147, 471-479. https://doi.org/10.1016/j.ecoenv.2017.09.007

Nauen, R., Ebbinghaus-Kintscher, U., Salgado, V.L., Kaussmann, M., 2003. Thiamethoxam is a neonicotinoid precursor converted to clothianidin in insects and plants. Pestic. Biochem. Physiol. 76, 55-69. https://doi.org/10.1016/S0048-3575(03)00065-8

Nicolson, S.W., Nepi, M., Pacini, E., 2007. Nectaries and nectar. Springer, Dordrecht.

Noyes, J.S., 2021. Universal Chalcidoidea Database. World Wide Web electronic publication. URL https://www.nhm.ac.uk/our-science/data/chalcidoids/

Nuyttens, D., Devarrewaere, W., Verboven, P., Foqué, D., 2013. Pesticide-laden dust emission and drift from treated seeds during seed drilling: A review. Pest Manag. Sci. 69, 564-575. https://doi.org/10.1002/ps.3485

O’Brien, P.J., Abdel-Aal, Y.A., Ottea, J.A., Graves, J.B., 1992. Relationship of insecticide resistance to carboxylesterases in Aphis gossypii (Homoptera: Aphididae) from Midsouth Cotton. J. Econ. Entomol. 85, 651-657. https://doi.org/10.1093/jee/85.3.651

Obok, E., Wetten, A., Allainguillaume, J., 2018. Electropenetrography application and molecular-based virus detection in mealybug (Hemiptera: Pseudococcidae) vectors of Cacao swollen shoot virus on Theobroma cacao L. Ann. Agric. Sci. 63, 55-65. https:// doi.org/10.1016/j.aoas.2018.04.004 
Ollerton, J., Erenler, H., Edwards, M., Crockett, R., 2014. Extinctions of aculeate pollinators in Britain and the role of large-scale agricultural changes. Science 346, 1360-1362. https://doi.org/10.1126/science.1257259

Ollerton, J., Winfree, R., Tarrant, S., 2011. How many flowering plants are pollinated by animals? Oikos 120, 321-326. https://doi.org/10.1111/j.1600-0706.2010.18644.x

Omer, A.D., Leigh, T.F., Granett, J., 1992. Insecticide resistance in field populations of greenhouse whitefly (Homoptera: Aleyrodidae) in the San Joaquin Valley (California) cotton cropping system. J. Econ. Entomol. 85, 21-27. https://doi.org/10.1093/ jee/85.1.21

Owusu, E.O., Horiike, M., Hirano, C., 1996. Polyacrylamide gel electrophoretic assessments of esterases in cotton aphid (Homoptera: Aphididae) resistance to dichlorvos. J. Econ. Entomol. 89, 302-306. https://doi.org/10.1093/jee/89.2.302

Oya, S., 1980. Feeding habits and honeydew components of the green rice leafhopper, Nephotettix cincticeps Uhler (Hemiptera: Deltocephalidae). Appl. Entomol. Zool. 15, 392-399.

Pan, Y., Yang, C., Gao, X., Peng, T., Bi, R., Xi, J., Xin, X., Zhu, E., Wu, Y., Shang, Q., 2015. Spirotetramat resistance adaption analysis of Aphis gossypii Glover by transcriptomic survey. Pestic. Biochem. Physiol. 124, 73-80. https://doi.org/10.1016/j. pestbp.2015.04.007

Pappas, M.L., Migkou, F., Broufas, G.D., 2013. Incidence of resistance to neonicotinoid insecticides in greenhouse populations of the whitefly, Trialeurodes vaporariorum (Hemiptera: Aleyrodidae) from Greece. Appl. Entomol. Zool. 48, 373-378. https:// doi.org/10.1007/s13355-013-0197-z

Pearsons, K.A., Rowen, E.K., Elkin, K.R., Wickings, K., Smith, R.G., Tooker, J.F., 2021. Small-grain cover crops have limited effect on neonicotinoid contamination from seed coatings. Environ. Sci. Technol. https://doi.org/10.1021/acs.est.0c05547

Pekas, A., De Craecker, I., Boonen, S., Wäckers, F.L., Moerkens, R., 2020. One stone; two birds: concurrent pest control and pollination services provided by aphidophagous hoverflies. Biol.Control 149, 104328. https://doi.org/10.1016/j.biocontrol.2020.104328

Pekas, A., Tena, A., Aguilar, A., Garcia-Marí, F., 2011. Spatio-temporal patterns and interactions with honeydew-producing Hemiptera of ants in a Mediterranean citrus orchard. Agric. For. Entomol. 13, 89-97. https://doi.org/10.1111/j.14619563.2010.00501.x

Pérez, C.J., Alvarado, P., Narváez, C., Miranda, F., Hernández, L., Vanegas, H., Hruska, A., Shelton, A.M., 2000. Assessment of insecticide resistance in five insect pests attacking field and vegetable crops in Nicaragua. J. Econ. Entomol. 93, 1779-1787. https://doi. org/10.1603/0022-0493-93.6.1779 
Phillips, B.B., Shaw, R.F., Holland, M.J., Fry, E.L., Bardgett, R.D., Bullock, J.M., Osborne, J.L., 2018. Drought reduces floral resources for pollinators. Glob. Chang. Biol. 24, 3226-3235. https://doi.org/10.1111/gcb.14130

Pielou, D.P., Downing, R.S., 1960. Dimethoate, a systemic of low mammalian toxicity, as an orchard insecticide in British Columbia. J. Entomol. Soc. Br. Columbia 57, 52-57.

Pisa, L.W., Amaral-Rogers, V., Belzunces, L.P., Bonmatin, J.M., Downs, C.A., Goulson, D., Kreutzweiser, D.P., Krupke, C., Liess, M., Mcfield, M., Morrissey, C.A., Noome, D.A., Settele, J., Simon-Delso, N., Stark, J.D., Van Der Sluijs, J.P., Van Dyck, H., Wiemers, M., 2015. Effects of neonicotinoids and fipronil on non-target invertebrates. Environ. Sci. Pollut. Res. 22, 68-102. https://doi.org/10.1007/s11356-014-3471-x

Planes, L., Catalan, J., Tena, A., Porcuna, J.L., Jacas, J.A., Izquierdo, J., Urbaneja, A., 2013. Lethal and sublethal effects of spirotetramat on the mealybug destroyer, Cryptolaemus montrouzieri. J. Pest Sci. 86, 321-327. https://doi.org/10.1007/s10340-012-0440-3

Potts, S.G., Biesmeijer, J.C., Kremen, C., Neumann, P., Schweiger, O., Kunin, W.E., 2010. Global pollinator declines: trends, impacts and drivers. Trends Ecol. Evol. 25, 345353. https://doi.org/10.1016/j.tree.2010.01.007

Powney, G.D., Carvell, C., Edwards, M., Morris, R.K.A., Roy, H.E., Woodcock, B.A., Isaac, N.J.B., 2019. Widespread losses of pollinating insects in Britain. Nat. Commun. 10, 1018. https://doi.org/10.1038/s41467-019-08974-9

Prabhaker, N., Gispert, C., Castle, S.J., 2012. Baseline susceptibility of Planococcus ficus (Hemiptera: Pseudococcidae) from California to select insecticides. J. Econ. Entomol. 105, 1392-1400. https://doi.org/10.1603/ec11340

Prabhaker, N., Toscano, N.C., Castle, S.J., Henneberry, T.J., 1997. Selection for imidacloprid resistance in silverleaf whiteflies from the imperial valley and development of a hydroponic bioassay for resistance monitoring. Pestic. Sci. 51, 419-428. https://doi. org/10.1002/(SICI)1096-9063(199712)51:4<419::AID-PS658>3.0.CO;2-L

Pringle, K.L., Giliomee, J.H., Addison, M.F., 1994. Vamidothion tolerance in a strain of the woolly apple aphid, Eriosoma lanigerum (Hausmann) (Hemiptera: Aphididae). African Entomol. 2, 123-125.

Punyawattoe, P., Han, Z., Sriratanasak, W., Arunmit, S., Chaiwong, J., Bullangpoti, V., 2013. Ethiprole resistance in Nilaparvata lugens (Hemiptera: Delphacidae): Possible mechanisms and cross-resistance. Appl. Entomol. Zool. 48, 205-211. https://doi. org/10.1007/s13355-013-0174-6

Qiong, R., Yong-Hua, X.U., Chen, L., Zhang, H.-Y., Jones, C.M., Devine, G.J., Gorman, K., Denholm, I., 2012. Characterisation of neonicotinoid and pymetrozine resistance in strains of Bemisia tabaci (Hemiptera: Aleyrodidae) from China. J. Integr. Agric. 11, 321-326. https://doi.org/10.1016/S2095-3119(12)60016-1 
Quesada, C.R., Scharf, M.E., Sadof, C.S., 2020. Excretion of non-metabolized insecticides in honeydew of striped pine scale. Chemosphere 249, 126167. https://doi.org/10.1016/j. chemosphere.2020.126167

Qureshi, J.A., Kostyk, B.C., Stansly, P.A., 2014. Insecticidal suppression of asian citrus psyllid Diaphorina citri (Hemiptera: Liviidae) vector of huanglongbing pathogens. PLoS One 9, e112331. https://doi.org/10.1371/journal.pone.0112331

Rader, R., Bartomeus, I., Garibaldi, L.A., Garratt, M.P.D., Howlett, B.G., Winfree, R., Cunningham, S.A., Mayfield, M.M., Arthur, A.D., Andersson, G.K.S., Bommarc, R., Brittain, C., Carvalheiro, L.G., Chacoff, N.P., Entling, M.H., Foully, B., Freitas, B.M., Gemmill-Herren, B., Ghazoul, J., Griffin, S.R., Gross, C.L., Herbertsson, L., Herzog, F., Hipólito, J., Jaggar, S., Jauker, F., Klein, A.-M., Kleijn, D., Krishnan, S., Lemos, C.Q., Lindström, S.A.M., Mandelik, Y., Monteiro, V.M., Nelson, W., Nilssonl, L., Pattemore, D.E., Pereira, N. de O., Pisanty, G., Potts, S.G., Reemer, M., Rundlöf, M., Sheffield, C.S., Scheper, J., Schüepp, C., Smith, H.G., Stanley, D.A., Stout, J.C., Szentgyörgy, H., Taki, H., Vergara, C.H., Viana, B.F., Woyciechowski, M., 2015. Non-bee insects are important contributors to global crop pollination. Proc. Natl. Acad. Sci. U.S.A. 113, 146-151. https://doi.org/10.1073/pnas.1517092112

Radja, K.H., Mikani, A., Mosallanejad, H., 2019. Biochemical resistance mechanisms to dimethoate in cabbage aphid Brevicoryne brassicae (L.) (Hom.: Aphididae). J. Agric. Sci. Technol. 22, 187-196.

Ragsdale, D.W., Landis, D.A., Brodeur, J., Heimpel, G.E., Desneux, N., 2011. Ecology and management of the soybean aphid in North America. Annu. Rev. Entomol. 56, 375-399. https://doi.org/10.1146/annurev-ento-120709-144755

Rand, T.A., Waters, D.K., 2020. Aphid honeydew enhances parasitoid longevity to the same extent as a high-quality floral resource: implications for conservation biological control of the wheat stem sawfly (Hymenoptera: Cephidae). J. Econ. Entomol. 113, 2022-2025. https://doi.org/10.1093/jee/toaa076

Rao, C.N., George, A., Rahangadale, S., 2018. Monitoring of resistance in field populations of Scirtothrips dorsalis (Thysanoptera: Thripidae) and Diaphorina citri (Hemiptera: Liviidae) to commonly used insecticides in citrus in Central India. J. Econ. Entomol. 112, 324-328. https://doi.org/10.1093/jee/toy311

Resh, V.H., Cardé, R.T., 2009. Encyclopedia of Insects. Academic Press publications. London. https://doi.org/10.1016/B978-0-12-374144-8.X0001-X

Rexrode, M., Barrett, M., Ellis, J., Gabe, P., Vaughan, A., Felkel, J., Melendez, J., 2003. EFED risk assessment for the seed treatment of clothianidin 600FS on corn and canola. United States Environmental Protection Agency, 20. 
Rezk, M., Hassan, A.N.T., El-Deeb, M.F., Shaarawy, N., Dewer, Y., 2019. The impact of insecticides on the cotton mealybug, Phenacoccus solenopsis (Tinsley): efficacy on potato, a new record of host plant in Egypt. J. Plant Prot. Res. 59, 50-59. https://doi. org/10.10.24425/jppr.2019.126042

Ricupero, M., Abbes, K., Haddi, K., Kurtulus, A., Desneux, N., Russo, A., Siscaro, G., Biondi, A., Zappalà, L., 2020. Combined thermal and insecticidal stresses on the generalist predator Macrolophus pygmaeus. Sci. Total Environ. 104743. https://doi. org/10.1016/j.phrs.2020.104743

Rogers, C.E., 1985. Extrafloral Nectar : Entomological Implications. Bull. Entomol. Soc. Am. 31, 15-20.

Rogers, M.A., Krischik, V.A., Martin, L.A., 2007. Effect of soil application of imidacloprid on survival of adult green lacewing, Chrysoperla carnea (Neuroptera: Chrysopidae), used for biological control in greenhouse. Biol. Control 42, 172-177. https://doi. org/10.1016/j.biocontrol.2007.05.006

Rondeau, G., Sánchez-Bayo, F., Tennekes, H.A., Decourtye, A., Ramírez-Romero, R., Desneux, N., 2015. Delayed and time-cumulative toxicity of imidacloprid in bees, ants and termites. Sci. Rep. 4, 5566. https://doi.org/10.1038/srep05566

Roy, D., Bhattacharjee, T., Biswas, A., Ghosh, A., Sarkar, S., Mondal, D., Sarkar, P.K., 2019. Resistance monitoring for conventional and new chemistry insecticides on Bemisia tabaci genetic group Asia-I in major vegetable crops from India. Phytoparasitica 47, 55-66. https://doi.org/10.1007/s12600-018-00707-w

Rundlöf, M., Andersson, G.K.S., Bommarco, R., Fries, I., Hederström, V., Herbertsson, L., Jonsson, O., Klatt, B.K., Pedersen, T.R., Yourstone, J., Smith, H.G., 2015. Seed coating with a neonicotinoid insecticide negatively affects wild bees. Nature 521, 7780. https://doi.org/10.1038/nature14420

Sanchez-Bayo, F., 2014. The trouble with neonicotinoids. Science 346, 806-807. https:// doi.org/10.1126/science.1259159

Sánchez-Bayo, F., Belzunces, L., Bonmatin, J.M., 2017. Lethal and sublethal effects, and incomplete clearance of ingested imidacloprid in honey bees (Apis mellifera). Ecotoxicology 26, 1199-1206. https://doi.org/10.1007/s10646-017-1845-9

Sanchez-Bayo, F., Goka, K., 2014. Pesticide residues and bees - A risk assessment. PLoS One 9, e94482. https://doi.org/10.1371/journal.pone.0094482

Sánchez-Bayo, F., Tennekes, H.A., Goka, K., 2013. Impact of systemic insecticides on organisms and ecosystems, in: Insecticides - development of safer and more effective technologies. Trdan S, editor, pp. 365-416. https://doi.org/10.1016/j.colsurfa.2011.12.014

Sánchez-Bayo, F., Wyckhuys, K.A.G., 2019. Worldwide decline of the entomofauna: A review of its drivers. Biol. Conserv. 232, 8-27. https://doi.org/10.1016/j.biocon.2019.01.020 
Sanchez Bayo, F., Tennekes, H.A., 2017. Assessment of ecological risks of agrochemicals requires a new framework. Environ. Risk Assess. Remediat. 1, 20-28. https://doi. org/10.4066/2529-8046.100025

Saska, P., Skuhrovec, J., Lukáš, J., Vlach, M., Chi, H., Tuan, S.J., Honek, A., 2017. Treating prey with glyphosate does not alter the demographic parameters and predation of the Harmonia axyridis (Coleoptera: Coccinellidae). J. Econ. Entomol. 110, 392-399. https://doi.org/10.1093/jee/tow325

Sawicki, R.M., Rice, A.D., 1978. Response of susceptible and resistant peach-potato aphids Myzus persicae (Sulz.) to insecticides in leaf-dip bioassays. Pestic. Sci. 9, $513-$ 516. https://doi.org/10.1002/ps.2780090604

Seagraves, M.P., Lundgren, J.G., 2012. Effects of neonicotinoid seed treatments on soybean aphid and its natural enemies. J. Pest Sci. 85, 125-132. https://doi.org/10.1007/s10340011-0374-1

Seebens, H., Blackburn, T.M., Dyer, E.E., Genovesi, P., Hulme, P.E., Jeschke, J.M., Pagad, S., Pyšek, P., Winter, M., Arianoutsou, M., Bacher, S., Blasius, B., Brundu, G., Capinha, C., Celesti-Grapow, L., Dawson, W., Dullinger, S., Fuentes, N., Jäger, H., Kartesz, J., Kenis, M., Kreft, H., Kühn, I., Lenzner, B., Liebhold, A., Mosena, A., Moser, D., Nishino, M., Pearman, D., Pergl, J., Rabitsch, W., Rojas-Sandoval, J., Roques, A., Rorke, S., Rossinelli, S., Roy, H.E., Scalera, R., Schindler, S., Štajerová, K., TokarskaGuzik, B., Van Kleunen, M., Walker, K., Weigelt, P., Yamanaka, T., Essl, F., 2017. No saturation in the accumulation of alien species worldwide. Nat. Commun. 8, 14435. https://doi.org/10.1038/ncomms14435

Serghiou, C.S., 1983. The citrus mealybug , Planococcus Citri Risso Carob Moth , Ectomyelois Ceratoniae Zeller, pest complex on grapefruit and its chemical control. Technical bulletin-Agricultural Research Institute.

Sgolastra, F., Medrzycki, P., Bortolotti, L., Renzi, M.T., Tosi, S., Bogo, G., Teper, D., Porrini, C., Molowny-Horas, R., Bosch, J., 2017. Synergistic mortality between a neonicotinoid insecticide and an ergosterol-biosynthesis-inhibiting fungicide in three bee species. Pest Manag. Sci. 73, 1236-1243. https://doi.org/10.1002/ps.4449

Sgolastra, F., Renzi, T., Draghetti, S., Medrzycki, P., Lodesani, M., Maini, S., Porrini, C., 2012. Effects of neonicotinoid dust from maize seed-dressing on honey bees. Bull. Insectology 65, 273-280. https://doi.org/10.5073/jka.2012.437.012

Shaaban, B., Seeburger, V., Schroeder, A., Lohaus, G., 2020. Sugar, amino acid and inorganic ion profiling of the honeydew from different hemipteran species feeding on Abies alba and Picea abies. PLoS One 15, e0228171. https://doi.org/10.1371/journal.pone.0228171 Shang, Q., Pan, Y., Fang, K., Xi, J., Brennan, J.A., 2011. Biochemical characterization of acetylcholinesterase, cytochrome $\mathrm{P} 450$ and cross-resistance in an omethoate-resistant 
strain of Aphis gossypii Glover. Crop Prot. 31, 15-20. https://doi.org/10.1016/j. cropro.2011.09.014

Shawki, M.A.-A., Titěra, D., Kazda, J., Kohoutková, J., Táborský, V., 2018. Toxicity to honeybees of water guttation and dew collected from winter rape treated with Nurelle $D^{\circledR}$. Plant Prot. Sci. 42, 9-14. https://doi.org/10.17221/2690-pps

Shen, G., Hu, X., Hu, Y., 2009. Kinetic study of the degradation of the insecticide pymetrozine in a vegetable-field ecosystem. J. Hazard. Mater. 164, 497-501. https:// doi.org/10.1016/j.jhazmat.2008.08.020

Shi, X., Jiang, L., Wang, H., Qiao, K., Wang, D., Wang, K., 2011. Toxicities and sublethal effects of seven neonicotinoid insecticides on survival, growth and reproduction of imidacloprid-resistant cotton aphid, Aphis gossypii. Pest Manag. Sci. 67, 1528-1533. https://doi.org/10.1002/ps.2207

Silva, V., Mol, H.G.J., Zomer, P., Tienstra, M., Ritsema, C.J., Geissen, V., 2019. Pesticide residues in European agricultural soils - A hidden reality unfolded. Sci. Total Environ. 653, 1532-1545. https://doi.org/10.1016/j.scitotenv.2018.10.441

Simon-Delso, N., Amaral-Rogers, V., Belzunces, L.P., Bonmatin, J.M., Chagnon, M., Downs, C., Furlan, L., Gibbons, D.W., Giorio, C., Girolami, V., Goulson, D., Kreutzweiser, D.P., Krupke, C.H., Liess, M., Long, E., McField, M., Mineau, P., Mitchell, E.A.D., Morrissey, C.A., Noome, D.A., Pisa, L., Settele, J., Stark, J.D., Tapparo, A., Van Dyck, H., Van Praagh, J., Van der Sluijs, J.P., Whitehorn, P.R., Wiemers, M., 2014. Systemic insecticides (neonicotinoids and fipronil): trends, uses, mode of action and metabolites. Environ. Sci. Pollut. Res. 22, 5-34. https://doi.org/10.1007/s11356-0143470-y

Singh, A., Mayer, V.E., Zytynska, S.E., Hesse, B., Weisser, W.W., 2021. The efficiency of plant defense: aphid pest pressure does not alter production of food rewards by okra plants in ant presence. Front. Plant Sci. 12, 1-13. https://doi.org/10.3389/fpls.2021.627570

Singh, S., 2016. Guttation: mechanism, momentum and modulation. Bot. Rev. 82, 149182. https://doi.org/10.1007/s12229-016-9165-y

Singh, S., Singh, T.N., 2013. Guttation 1: chemistry, crop husbandry and molecular farming. Phytochem. Rev. 12, 147-172. https://doi.org/10.1007/s11101-012-9269-x

Siviter, H., Brown, M.J.F., Leadbeater, E., 2018. Sulfoxaflor exposure reduces bumblebee reproductive success. Nature. 561, 109-112 https://doi.org/10.1038/s41586-018-0430-6

Siviter, H., Muth, F., 2020. Do novel insecticides pose a threat to beneficial insects? Proc. R. Soc. B Biol. Sci. 287, 20201265. https://doi.org/10.1098/rspb.2020.1265

Smith, D., Hinz, H., Mulema, J., Weyl, P., Ryan, M.J., 2018a. Biological control and the Nagoya Protocol on access and benefit sharing-a case of effective due diligence. Biocontrol Sci. Technol. 28, 914-926. https://doi.org/10.1080/09583157.2018.1460317 
Smith, J.L., Baute, T.S., Schaafsma, A.W., 2020. Quantifying early-season pest injury and yield protection of insecticide seed treatments in corn and soybean production in Ontario, Canada. J. Econ. Entomol. 113, 2197-2212. https://doi.org/10.1093/jee/toaa132 Spiller, N.J., Koenders, L., Tjallingii, W.F., 1990. Xylem ingestion by aphids - a strategy for maintaining water balance. Entomol. Exp. Appl. 55, 101-104. https://doi. org/10.1111/j.1570-7458.1990.tb01352.x

Srigiriraju, L., Semtner, P.J., Anderson, T.D., Bloomquist, J.R., 2009. Esterase-based resistance in the tobacco-adapted form of the green peach aphid, Myzus persicae (Sulzer) (Hemiptera: Aphididae) in the Eastern United States. Arch. Insect Biochem. Physiol. 72, 105-123. https://doi.org/10.1002/arch.20326

Stanley, D.A., Smith, K.E., Raine, N.E., 2015. Bumblebee learning and memory is impaired by chronic exposure to a neonicotinoid pesticide. Sci. Rep. 5, 16508. https:// doi.org/10.1038/srep16508

Stapel, J.O., Cortesero, A.M., De Moraes, C.M., Tumlinson, J.H., Lewis, W.J., 1997. Extrafloral nectar, honeydew, and sucrose effects on searching behavior and efficiency of Microplitis croceipes (Hymenoptera: Braconidae) in cotton. Environ. Entomol. 26, 617-623. https://doi.org/10.1093/ee/26.3.617

Stapel, J.O., Cortesero, A.M., Lewis, W.J., 2000. Disruptive sublethal effects of insecticides on biological control: altered foraging ability and life span of a parasitoid after feeding on extrafloral nectar of cotton treated with systemic insecticides. Biol. Control 17, 243-249. https://doi.org/10.1006/bcon

Starner, K., Goh, K.S., 2012. Detections of the neonicotinoid insecticide imidacloprid in surface waters of three agricultural regions of California, USA, 2010-2011. Bull. Environ. Contam. Toxicol. 88, 316-321. https://doi.org/10.1007/s00128-011-0515-5

Stehle, S., Schulz, R., 2015. Agricultural insecticides threaten surface waters at the global scale. Proc. Natl. Acad. Sci. U. S. A. 112, 5750-5755. https://doi.org/10.1073/ pnas. 1500232112

Steppuhn, A., Wäckers, F.L., 2004. HPLC sugar analysis reveals the nutritional state and the feeding history of parasitoids. Funct. Ecol. 18, 812-819. https://doi.org/10.1111/ j.0269-8463.2004.00920.x

Stoner, K.A., Eitzer, B.D., 2012. Movement of soil-applied imidacloprid and thiamethoxam into nectar and pollen of squash (Cucurbita pepo). PLoS One 7, e39114. https://doi. org/10.1371/journal.pone.0039114

Struger, J., Grabuski, J., Cagampan, S., Sverko, E., Marvin, C., 2016. Occurrence and distribution of carbamate pesticides and metalaxyl in Southern Ontario surface waters 2007-2010. Bull. Environ. Contam. Toxicol. 96, 423-431. https://doi.org/10.1007/ s00128-015-1719-x 
Sudderuddin, K.I., 1973. Studies of insecticide resistance in Myzus persicae (Sulz.) (Hem., Aphididae). Bull. Entomol. Res. 62, 533-539. https://doi.org/10.1017/ S0007485300005411 Syngenta, 2020.

Syngenta, 2020. Pymetrozine (Plenum). URL: https://www.agroterra.com/p/plenuminsecticida-sistemico-syngenta/3108589

Syngenta, 2019. Thiamethoxam (Actara). URL https://www.syngenta.es/product/cropprotection/insecticida/actara-25-wg.

Szeto, S.Y., Vernon, R.S., Brown, M.J., 1985. Degradation of dimethoate and pirimicarb in asparagus. J. Agric. Food Chem. 33, 763-767. https://doi.org/10.1021/jf00064a051

Tamaš, N., Dojnov, B., Margetić, A., Vujčić, M., Špirović, B., Miletić, N., Stević, M., Vujčić, Z., 2015. Resistance to common organophosphate and carbamate insecticides in Aphis pomi (Hemiptera: Aphididae). Fruits 70, 135-142. https://doi.org/10.1051/ fruits/2015005

Tang, L. De, Wu, J.H., Ali, S., Ren, S.X., 2013. Establishment of baseline toxicity data to different insecticides for Aphis craccivora Koch and Rhopalosiphum maidis (Fitch) (Homoptera: Aphididae) by glass tube residual film technique. Pak. J. Zool. 45, 411-415.

Tang, Q.L., Ma, K.S., Hou, Y.M., Gao, X.W., 2017. Monitoring insecticide resistance and diagnostics of resistance mechanisms in the green peach aphid, Myzus persicae (Sulzer) (Hemiptera: Aphididae) in China. Pestic. Biochem. Physiol. 143, 39-47. https://doi.org/10.1016/j.pestbp.2017.09.013

Tappert, L., Pokorny, T., Hofferberth, J., Ruther, J., 2017. Sublethal doses of imidacloprid disrupt sexual communication and host finding in a parasitoid wasp. Sci. Rep. 7, 42756. https://doi.org/10.1038/srep42756

Tasman, K., Hidalgo, S., Zhu, B., Rands, S.A., Hodge, J.J.L., 2021. Neonicotinoids disrupt memory, circadian behaviour and sleep. Sci. Rep. 11, 2061. https://doi.org/10.1038/ s41598-021-81548-2

Taylor, S. V., Burrack, H.J., Michael Roe, R., Bacheler, J.S., Sorenson, C.E., 2015. Systemic imidacloprid affects intraguild parasitoids differently. PLoS One 10, e0144598. https:// doi.org/10.1371/journal.pone.0144598

Teicher, H.B., 2017. Pesticide formulation, in: Pesticides \& Biopesticides Formulation \& Mode of Action. Biocomm Press, Denmark. pp. 59-85.

Tena, A., Llácer, E., Urbaneja, A., 2013a. Biological control of a non-honeydew producer mediated by a distinct hierarchy of honeydew quality. Biol. Control 67, 117-122. https://doi.org/10.1016/j.biocontrol.2013.07.018

Tena, A., Pekas, A., Wäckers, F.L., Urbaneja, A., 2013b. Energy reserves of parasitoids depend on honeydew from non-hosts. Ecol. Entomol. 38, 278-289. https://doi. org/10.1111/een.12018 
Tena, A., Senft, M., Desneux, N., Dregni, J., Heimpel, G.E., 2018. The influence of aphidproduced honeydew on parasitoid fitness and nutritional state: A comparative study. Basic Appl. Ecol. 29, 55-68. https://doi.org/10.1016/j.baae.2018.04.003

Tena, A., Wäckers, F.L., Heimpel, G.E., Urbaneja, A., Pekas, A., 2016. Parasitoid nutritional ecology in a community context: the importance of honeydew and implications for biological control. Curr. Opin. Insect Sci. 14, 100-104. https://doi.org/10.1016/j. cois.2016.02.008

Thomas, J.A., Telfer, M.G., Roy, D.B., Preston, C.D., Greenwood, J.J.D., Asher, J., Fox, R., Clarke, R.T., Lawton, J.H., 2004. Comparative losses of British butterflies, birds, and plants and the global extinction crisis. Science 303, 1879-1881. https://doi. org/10.1126/science.1095046

Thompson, H.M., 2001. Assessing the exposure and toxicity of pesticides to bumblebees (Bombus sp .). Apidologie 32, 305-321. https://doi.org/10.1051/apido:2001131

Tilmon, K.J., Hodgson, E.W., O’Neal, M.E., Ragsdale, A.D.W., 2011. Biology of the soybean aphid, Aphis glycines (Hemiptera: Aphididae) in the United States invasion history and distribution. J. Integr. Pest Manag. 2, A1-A7. https://doi.org/10.1603/ IPM10016

Tingle, C.C.D., Rother, J.A., Dewhurst, C.F., Lauer, S., King, W.J., 2003. Fipronil: environmental fate, ecotoxicology, and human health concerns. Rev. Environ. Contam. Toxicol. 176, 1-66. https://doi.org/10.1007/978-1-4899-7283-5_1

Tomizawa, M., Casida, J.E., 2005. Neonicotinoid insecticide toxicology: mechanisms of selective action. Annu. Rev. Pharmacol. Toxicol. 45, 247-268. https://doi.org/10.1146/ annurev.pharmtox.45.120403.095930

Tooker, J.F., Douglas, M.R., Krupke, C.H., 2017. Neonicotinoid seed treatments: limitations and compatibility with Integrated Pest Management. Agric. Environ. Lett. 2. https://doi.org/10.2134/ael2017.08.0026

Tooker, J.F., Pearsons, K., 2021. Newer characters, same story: neonicotinoid insecticides disrupt food webs through direct and indirect effects. Curr. Opin. Insect Sci. S22145745, 00021-3. https://doi.org/https://doi.org/10.1016/j.cois.2021.02.013

Tooming, E., Merivee, E., Must, A., Merivee, M.I., Sibul, I., Nurme, K., Williams, I.H., 2017. Behavioural effects of the neonicotinoid insecticide thiamethoxam on the predatory insect Platynus assimilis. Ecotoxicology 26, 902-913. https://doi.org/10.1007/s10646-017-1820-5

Tosi, S., Nieh, J.C., 2019. Lethal and sublethal synergistic effects of a new systemic pesticide, flupyradifurone (Sivantow), on honeybees. Proc. R. Soc. B Biol. Sci. 286, 20190433. https://doi.org/10.1098/rspb.2019.0433

Tran, D.H., Takagi, M., Takasu, K., 2004. Effects of selective insecticides on host searching and oviposition behavior of Neochrysocharis formosa (Westwood) (Hymenoptera: 
Eulophidae), a larval parasitoid of the American serpentine leafminer. Appl. Entomol. Zool. 39, 435-441. https://doi.org/10.1303/aez.2004.435

Tsvetkov, N., Samson-Robert, O., Sood, K., Patel, H.S., Malena, D.A., Gajiwala, P.H., Maciukiewicz, P., Fournier, V., Zayed, A., 2017. Chronic exposure to neonicotinoids reduces honey bee health near corn crops. Science 356, 1395-1397. https://doi. org/10.1126/science.aam7470

Ülgentürk, S., Cosic, B., Özdemir, I., İpek, A., Sorkun, K., 2020. Honeydew producing insects in some forests of turkey and their potential to produce of honeydew honey. Balt. For. 26, 125-131. https://doi.org/10.46490/BF397

University of California, 2021. Agriculture: Pest Management Guidelines. Cotton. URL http://ipm.ucanr.edu/PMG/r114300111.html

University of Herthfordshire, 2021. Pesticide Properties DataBase. URL https://sitem. herts.ac.uk/aeru/footprint/es/Reports/562.htm\#trans

Urbaneja-Bernat, P., Hernández-Suárez, E., Tena, A., Urbaneja, A., 2020a. Preventive measures to limit the spread of Trioza erytreae (Del Guercio) (Hemiptera: Triozidae) in mainland Europe. J. Appl. Entomol. 144, 553-559. https://doi.org/10.1111/jen.12771

Urbaneja-Bernat, P., Pérez-Rodríguez, J., Krüger, K., Catalán, J., Rizza, R., HernándezSuárez, E., Urbaneja, A., Tena, A., 2019. Host range testing of Tamarixia dryi (Hymenoptera: Eulophidae) sourced from South Africa for classical biological control of Trioza erytreae (Hemiptera: Psyllidae) in Europe. Biol. Control 135, 110-116. https://doi.org/10.1016/j.biocontrol.2019.04.018

Urbaneja-Bernat, P., Tena, A., González-Cabrera, J., Rodriguez-Saona, C., 2020b. Plant guttation provides nutrient-rich food for insects. Proceedings. Biol. Sci. 287, 20201080. https://doi.org/10.1098/rspb.2020.1080

Urbaneja, A., Grout, T.G., Gravena, S., Wu, F., Cen, Y., Stansly, P.A., 2020. Citrus pests in a global world., The Genus Citrus. Elsevier Inc. https://doi.org/10.1016/B978-0-12812163-4.00016-4

Urbaneja, A., Pascual-Ruiz, S., Pina, T., Abad-Moyano, R., Vanaclocha, P., Montón, H., Dembilio, O., Castañera, P., Jacas, J.A., 2008. Efficacy of five selected acaricides against Tetranychus urticae (Acari: Tetranychidae) and their side effects on relevant natural enemies occurring in citrus orchards. Pest Manag. Sci. 64, 834-842.

US Climate Data, 2019. URL https://www.usclimatedata.com/climate/minneapolis/ minnesota/united-states/usmn0503.

Vacas, S., Navarro, I., Marzo, J., Navarro-Llopis, V., Primo, J., 2019. Sex pheromone of the invasive mealybug citrus pest, Delottococcus aberiae (Hemiptera:Pseudococcidae). A new monoterpenoid with a necrodane skeleton. J. Agric. Food Chem. 67, 9441-9449. https://doi.org/10.1021/acs.jafc.9b01443 
van den Brink, P.J., Van Smeden, J.M., Bekele, R.S., Dierick, W., De Gelder, D.M., Noteboom, M., Roessink, I., 2016. Acute and chronic toxicity of neonicotinoids to nymphs of a mayfly species and some notes on seasonal differences. Environ. Toxicol. Chem. 35, 128-133. https://doi.org/10.1002/etc.3152

van Leeuwen, T., Vontas, J., Tsagkarakou, A., Dermauw, W., Tirry, L., 2010. Acaricide resistance mechanisms in the two-spotted spider mite Tetranychus urticae and other important Acari: A review. Insect Biochem. Mol. Biol. 40, 563-572. https://doi. org/10.1016/j.ibmb.2010.05.008

van Lenteren, J.C., Bolckmans, K., Köhl, J., Ravensberg, W.J., Urbaneja, A., 2017. Biological control using invertebrates and microorganisms: plenty of new opportunities. BioControl 63, 39-59. https://doi.org/10.1007/s10526-017-9801-4

van Lexmond, M.B., Bonmatin, J.M., Goulson, D., Noome, D.A., 2015. Worldwide integrated assessment on systemic pesticides global collapse of the entomofauna: exploring the role of systemic insecticides. Environ. Sci. Pollut. Res. 22, 1-4. https:// doi.org/10.1007/s11356-014-3220-1

van Neerbos, F.A.C., de Boer, J.G., Salis, L., Tollenaar, W., Kos, M., Vet, L.E.M., Harvey, J.A., 2020. Honeydew composition and its effect on life-history parameters of hyperparasitoids. Ecol. Entomol. 45, 278-289. https://doi.org/10.1111/een.12799

van Scoy, A., Pennell, A., Zhang, X., 2016. Environmental fate and toxicology of dimethoate. Rev. Environ. Contam. Toxicol. 237, 53-70. https://doi.org/10.1007/978-3-319-23573-8_3 Vanaclocha, P., Vidal-Quist, C., Oheix, S., Montón, H., Planes, L., Catalán, J., Tena, A., Verdú, M.J., Urbaneja, A., 2013. Acute toxicity in laboratory tests of fresh and aged residues of pesticides used in citrus on the parasitoid Aphytis melinus. J. Pest Sci. 86, 329-336. https://doi.org/10.1007/s10340-012-0448-8

Vogel, G., 2017. Where have all the insects gone? Science. 356, 576-579. https://doi. org/10.1126/science.356.6338.576

Vosteen, I., Gershenzon, J., Kunert, G., 2016. Hoverfly preference for high honeydew amounts creates enemy-free space for aphids colonizing novel host plants. J. Anim. Ecol. 85, 1286-1297. https://doi.org/10.1111/1365-2656.12564

Wäckers, F.L., 2000. Do oligosaccharides reduce the suitability of honeydew for predators and parasitoids? A further facet to the function of insect-synthesized honeydew sugars. Oikos 90, 197-201. https://doi.org/10.1034/j.1600-0706.2000.900124.x

Wäckers, F.L., Steppuhn, A., 2003. Characterizing nutritional state and food source use of parasitoids collected in fields with high and low nectar availability. Int. Organ. Biol. Integr. Control Noxious Anim. Plants, West Palearct. Reg. Sect. Bull. 26, 203-208.

Wäckers, F.L., van Rijn, P.C.J., Bruin, J., 2005. Suitability of (extra-) floral nectar, pollen and honeydew as insect food sources, in: Plant-provided food for carnivorous insects: 
a protective mutualism and its applications. pp. 17-74. https://doi.org/10.1017/ CBO9780511542220.003

Wäckers, F.L., van Rijn, P.C.J., Heimpel, G.E., 2008. Honeydew as a food source for natural enemies: making the best of a bad meal? Biol. Control 45, 176-184. https:// doi.org/10.1016/j.biocontrol.2008.01.007

Wajnberg, E., Bernstein, C., Van Alphen, J., 2008. Behavioral ecology of insect parasitoids: from theoretical approaches to field applications. John Wiley \& Sons, Ltd.

Wang, Jin, F., Cao, X., Shao, Y., Wang, Jian, She, Y., Qi, Y., Zhang, C., Li, H., Jin, M., Wang, Jing, Shao, H., Zheng, L., 2018. Residue behaviors and risk assessment of flonicamid and its metabolites in the cabbage field ecosystem. Ecotoxicol. Environ. Saf. 161, 420429. https://doi.org/10.1016/j.ecoenv.2018.05.074

Wang, Z., Yao, M., Wu, Y., 2009. Cross-resistance, inheritance and biochemical mechanisms of imidacloprid resistance in B-biotype Bemisia tabaci. Pest Manag. Sci. 65, 1189-1194. https://doi.org/10.1002/ps.1808

Wang, Z.H., Gong, Y.J., Chen, J.C., Su, X.C., Cao, L.J., Hoffmann, A.A., Wei, S.J., 2018. Laboratory selection for resistance to sulfoxaflor and fitness costs in the green peach aphid Myzus persicae. J. Asia. Pac. Entomol. 21, 408-412. https://doi.org/10.1016/j. aspen.2018.01.024

Watanabe, H., Katayama, N., Yano, E., Sugiyama, R., Nishikawa, S., Endou, T., Watanabe, K., Takabayashi, J., Ozawa, R., 2014. Effects of aphid honeydew sugars on the longevity and fecundity of the aphidophagous gall midge Aphidoletes aphidimyza. Biol. Control 78, 55-60. https://doi.org/10.1016/j.biocontrol.2014.07.007

Way, M.J., 1963. Mutualism between ants and honeydew producing homopterans. Annu. Rev. Entomol. 8, 307-344. https://doi.org/10.1146/annurev.en.08.010163.001515

Weichel, L., Nauen, R., 2004. Uptake, translocation and bioavailability of imidacloprid in several hop varieties. Pest Manag. Sci. 60, 440-446. https://doi.org/10.1002/ps.831

Whitehorn, P.R., O’Connor, S., Wackers, F.L., Goulson, D., 2012. Neonicotinoid pesticide reduces bumble bee colony growth and queen production. Science 336, 351-352. https://doi.org/10.1126/science.1215025

Willow, J., Silva, A., Veromann, E., Smagghe, G., 2019. Acute effect of low-dose thiacloprid exposure synergised by tebuconazole in a parasitoid wasp. PLoS One 14, e0212456. https://doi.org/10.1371/journal.pone.0212456

Woodcock, B.A., Bullock, J.M., Shore, R.F., Heard, M.S., Pereira, M.G., Redhead, J., Ridding, L., Dean, H., Sleep, D., Henrys, P., Peyton, J., Hulmes, S., Hulmes, L., Sárospataki, M., Saure, C., Edwards, M., Genersch, E., Knäbe, S., Pywell, R.F., 2017. Country-specific effects of neonicotinoid pesticides on honey bees and wild bees. Science 356, 1393-1395. https://doi.org/10.1126/science.aaa1190 
Woodcock, B.A., Isaac, N.J.B., Bullock, J.M., Roy, D.B., Garthwaite, D.G., Crowe, A., Pywell, R.F., 2016. Impacts of neonicotinoid use on long-term population changes in wild bees in England. Nat. Commun. 7, 12459. https://doi.org/10.1038/ncomms12459 Worldbank, 2020. URL https://data.worldbank.org/indicator/AG.LND.AGRI.K2

Wyckhuys, K.A., Zhang, W., Prager, S.D., Kramer, D.B., Delaquis, E., Gonzalez, C.E., van der Werf, W., 2018. Biological control of an invasive pest eases pressures on global commodity markets. Environ. Res. Lett. 13, 094005. https://doi.org/10.1088/17489326/aad8f0

Wyckhuys, K.A.G., Strange-George, J.E., Kulhanek, C.A., Wäckers, F.L., Heimpel, G.E., 2008. Sugar feeding by the aphid parasitoid Binodoxys communis: How does honeydew compare with other sugar sources? J. Insect Physiol. 54, 481-491. https:// doi.org/10.1016/j.jinsphys.2007.11.007

Xi, J., Pan, Y., Bi, R., Gao, X., Chen, X., Peng, T., Zhang, M., Zhang, H., Hu, X., Shang, Q., 2015. Elevated expression of esterase and cytochrome P450 are related with lambdacyhalothrin resistance and lead to cross resistance in Aphis glycines Matsumura. Pestic. Biochem. Physiol. 118, 77-81. https://doi.org/10.1016/j.pestbp.2014.12.002

Yao, F., Zheng, Y., Zhao, J., Desneux, N., He, Y., Weng, Q., 2015. Lethal and sublethal effects of thiamethoxam on the whitefly predator Serangium japonicum (Coleoptera : Coccinellidae ) through different exposure routes. Chemosphere 128, 49-55. https:// doi.org/10.1016/j.chemosphere.2015.01.010

Yao, F.L., Zheng, Y., Huang, X.Y., Ding, X.L., Zhao, J.W., Desneux, N., He, Y.X., Weng, Q.Y., 2017. Dynamics of Bemisia tabaci biotypes and insecticide resistance in Fujian province in China during 2005-2014. Sci. Rep. 7, 40803. https://doi.org/10.1038/srep40803

Yao, I., Akimoto, S.I., 2001. Ant attendance changes the sugar composition of the honeydew of the drepanosiphid aphid Tuberculatus quercicola. Oecologia 128, 36-43. https://doi.org/10.1007/s004420100633

Yoo, J.K., Lee, S.W., Ahn, Y.J., Nagata, T., Shono, T., 2002. Altered acetylcholinesterase as a resistance mechanism in the brown planthopper (Homoptera: Delphacidae), Nilaparvata lugens Stål. Appl. Entomol. Zool. 37, 37-41. https://doi.org/10.1303/ aez.2002.37

Yu, B., Chen, Z., Lu, X., Huang, Y., Zhou, Y., Zhang, Q., Wang, D., Li, J., 2020. Effects on soil microbial community after exposure to neonicotinoid insecticides thiamethoxam and dinotefuran. Sci. Total Environ. 725. https://doi.org/10.1016/j.scitotenv.2020.138328

Zeddies, J., Schaab, R.P., Neuenschwander, P., Herren, H.R., 2001. Economics of biological control of cassava mealybug in Africa. Agric. Econ. 24, 209-219. https:// doi.org/10.1111/j.1574-0862.2001.tb00024.x 
Zeun, R., Scalliet, G., Oostendorp, M., 2013. Biological activity of sedaxane - a novel broad-spectrum fungicide for seed treatment. Pest Manag. Sci. 69, 527-534. https:// doi.org/10.1002/ps.3405

Zhang, L., Lu, H., Guo, K., Yao, S., Cui, F., 2017. Insecticide resistance status and detoxification enzymes of wheat aphids Sitobion avenae and Rhopalosiphum padi. Sci. China Life Sci. 60, 927-930. https://doi.org/10.1007/s11427-017-9105-x

Zhang, W., 2018. Global pesticide use: Profile, trend, cost / benefit and more. Proc. Int. Acad. Ecol. Environ. Sci. 8, 1-27.

Zioga, E., Kelly, R., White, B., Stout, J.C., 2020. Plant protection product residues in plant pollen and nectar: a review of current knowledge. Environ. Res. 189, 109873. https:// doi.org/10.1016/j.envres.2020.109873

Züst, T., Agrawal, A.A., 2015. Population growth and sequestration of plant toxins along a gradient of specialization in four aphid species on the common milkweed Asclepias syriaca. Funct. Ecol. 30, 547-556. https://doi.org/10.1111/1365-2435.12523

Zwick, R.W., Fields, G.J., 1978. Field and laboratory evaluations of fenvalerate against several insect and mite pests of apple and pear in Oregon. J. Econ. Entomol. 71, 793796. https://doi.org/10.1093/jee/71.5.793 



\section{Summary}
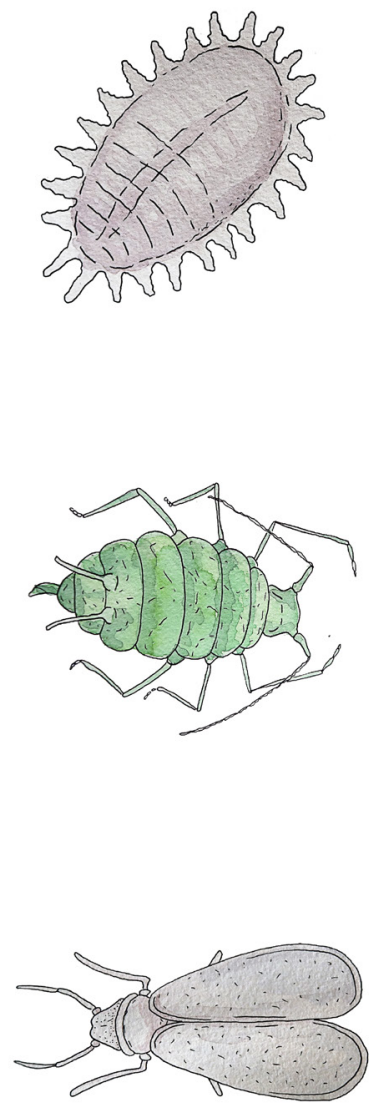

A myriad of beneficial insects such as pollinators and biological control agents need carbohydrates to meet their requirements for daily physical activities and metabolic processes. In agricultural lands, beneficial insects find carbohydrates mainly in nectar and honeydew. Nectar, the sugar produced by flowering plants to attract pollinators and thus accomplish reproduction, is often scarce in most agroecosystems because it is limited to the brief flowering period of the crop (if present). In addition, nectar is available in the spontaneous presence of flowering plants that appear along crop borders, ditches and roadsides, but these plants are often removed to avoid competition with the crop. Honeydew, the excretion product of many hemipterans such as aphids, whiteflies, coccids, mealybugs or psyllids, is on the contrary, highly abundant and accessible in agricultural lands throughout the year. For this reason, many beneficial insects rely on honeydew as a main carbohydrate source and some others feed on it in periods when nectar is scarce.

The use of systemic insecticides has been considered an excellent option for integrated pest management programs because once applied, they move systemically to all plant tissues, harming only herbivores that feed on the plant. Nevertheless, some systemic insecticides can reach plant-derived food sources such as nectar or pollen, and many insects that feed on them are exposed. Most environmental risk assessments have considered nectar and pollen as key routes of exposure to evaluate the ecological safety of systemic insecticides. The aim of my PhD thesis was to explore whether honeydew is a route of systemic insecticide exposure for beneficial insects. This route 
of exposure could be more impactful than the route of nectar because honeydew is ubiquitous in agroecosystems.

Neonicotinoids are the most widely used and toxic systemic insecticides. These insecticides are used in many crops against a broad-spectrum of insect pests, but they have been banned in Europe due to their toxic effect on pollinators. In chapter 2, I investigated whether honeydew excreted by mealybugs feeding on treated-trees contained neonicotinoids that could harm insects feeding on it. In this chapter, I applied the neonicotinoids thiamethoxam and imidacloprid into the soil of citrus plants infested with the mealybug Planococcus citri (Risso) (Hemiptera: Pseudococcidae) at the recommended doses to test one of the most common modes of applications. Additionally, I sprayed the neonicotinoids onto the leaves at half of the recommended dose to simulate other potential scenarios in which low doses of neonicotinoids can reach hemipterans. For both experiments, I collected honeydew from mealybugs feeding on trees treated with neonicotinoids during five days after the treatment and chemical analysis and bioassays were carried out with this honeydew. Imidacloprid and thiamethoxam were commonly present in the hemipteran honeydew at concentrations higher than those found in nectar. Furthermore, I carried out bioassays to study the toxicity of contaminated honeydew for beneficial organisms. Honeydew contaminated with neonicotinoids was harmful to the hoverfly Spaherophoria rueppellii (Wiedemann) (Diptera: Syrphidae) and the parasitic wasp Anagyrus vladimiri (Girault) (Hymenoptera: Encyrtidae). These results demonstrate that honeydew is a route of exposure to neonicotinoids for beneficial insects.

Recently, the European Commission banned the use of neonicotinoids in outdoor crops due to their high toxicity on non-target organisms and their high persistence in soil, water and plants. As a consequence, the use of neonicotinoids has been substituted by other less persistent insecticides such as flonicamid or pymetrozine. In other countries, these insecticides are recommended in IPM programs because they are considered selective and less toxic to beneficial insects than neonicotinoids. In chapter 3, I explored whether flonicamid and pymetrozine can reach honeydew excreted by the mealybug $P$. citri feeding on treated plants. We detected both systemic insecticides in the mealybug honeydew. Moreover, honeydew with flonicamid or pymetrozine was moderately toxic to S. rueppellii and harmless to A. vladimiri. The results of this chapter are important because pymetrozine and flonicamid are toxic to aphids and whiteflies, but mealybugs are tolerant to these insecticides, and may excrete honeydew contaminated with flonicamid or pymetrozine until the insecticide is degraded in the plant. Therefore, we expected 
that tolerant mealybugs excrete honeydew contaminated with flonicamid or pymetrozine during long periods when they are applied against whiteflies or aphids.

Seed coating is the leading delivery method of neonicotinoid insecticides in major crops such as soybean, wheat, cotton or maize. The protection period of coated seeds lasts approximately 3-4 weeks after planting, but the active ingredient remains in the plant for a long periods at low concentrations. Hemipterans colonize plants and may excrete honeydew with insecticides. In chapter 4, I demonstrate for the first time that neonicotinoids from soybean coated seeds reach honeydew excreted by the soybean aphid Aphis glycines Matsumura (Hemiptera: Aphididae) 30-40 days after sowing the seeds. This contaminated honeydew reduced the longevity of the biological control agents of the soybean aphid, the predatory midge Aphidoletes aphidimyza Rondani (Diptera: Cecidomyiidae) and the parasitic wasp Aphelinus certus Yanosh (Hymenoptera: Aphelinidae). The results of this chapter are important because environmental agencies are now evaluating the use of neonicotinoid-coated seeds and the data show that they should consider that these plants can hold hemipterans that excrete contaminated honeydew.

Several studies have demonstrated that beneficial insects do not discriminate between food sources uncontaminated and contaminated with neonicotinoids. In fact, it has been shown that some pollinator species prefer food with neonicotinoids. However, this has never been demonstrated for using honeydew as food source. In chapter 5, I carried out behavioral assays to study whether the parasitic wasp A. vladimiri and the hoverfly $S$. rueppellii can discriminate between uncontaminated honeydew or honeydew contaminated with either imidacloprid or thiamethoxam. Hoverflies did not discriminate between honeydew contaminated with insecticides or uncontaminated honeydew. Instead, parasitic wasps discriminated between uncontaminated honeydew or honeydew with thiamethoxam, but they preferred the contaminated food source. The results of this chapter may have important consequences because in the previous chapters, we collected uncontaminated honeydew and honeydew contaminated with systemic insecticides in samples from the same plant and day.

In chapter 6, I review in a perspective paper the importance of honeydew as a route of insecticide exposure to beneficial insects. First, I explored the potential pathways through which honeydew might be contaminated with insecticides; Second, I describe the hemipteran families that are more likely to excrete contaminated honeydew due to their different feeding behaviour. Third, I evaluate the systemic insecticides that are 
more likely to contaminate honeydew due to their physicochemical properties. Finally, I analyse several model crops where contaminated honeydew can be highly accessible for beneficial organisms and commonly contaminated with systemic insecticides.

In conclusion, my thesis describes a new route that is highly common, in which beneficial insects can be harmed by insecticides. In this thesis, I demonstrate that this route of exposure has different degrees of toxicity on beneficial insects that depend on: i) the active ingredient; ii) the mode of application of the insecticide; iii) the hemipteran species that excrete the honeydew; iv) the plant species; and v) the beneficial insect species tested. Therefore, this route of exposure is variable and complex and further studies are needed. In addition, I recommend including honeydew in environmental risk assessments, as it is likely to affect a wider range of beneficial insects than the route of contaminated nectar. 
Acknowledgements
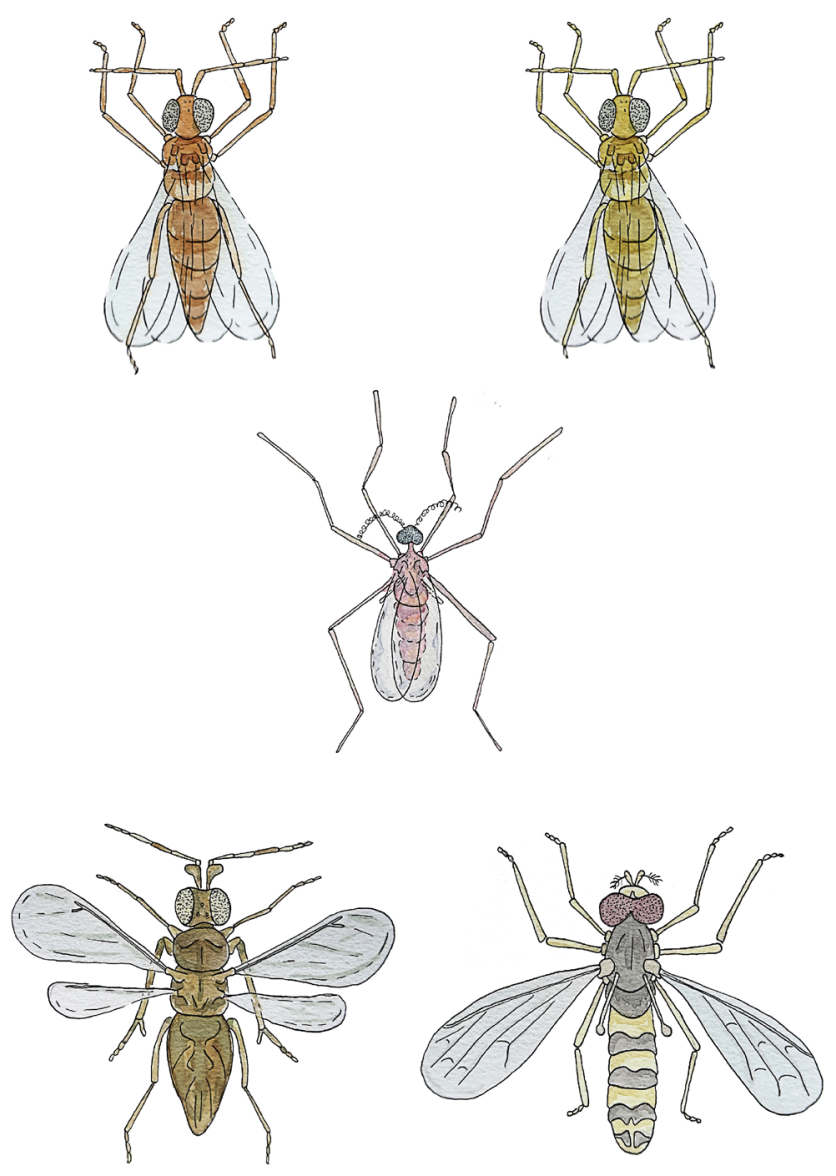

It seems like yesterday that I started this journey! I remember that when I submitted the $\mathrm{PhD}$ application I was working in a small village in Belgium. I had to cross this tiny country to submit my application at the embassy in Brussels. For many different reasons, I arrived there $2 \mathrm{~min}$ before closing. Those two minutes would have changed my life. Looking back, it is hard for me to think of a sweeter journey, more adventurous and fuller of learning than that of my $\mathrm{PhD}$. Now that this life chapter is coming to an end, it's time for me to acknowledge the people involved in the project or outsiders that have supported me.

In the first place, I want to thank my supervisors Marcel and Alejandro. Alejandro, you are a brilliant scientist, the perfect supervisor and a great person. You have given me confidence and freedom to discover this impactful route of exposure to beneficial insects. You have helped me to plan my PhD efficiently, and supported me when I went to Minnesota (you even visited me!). More importantly, when I arrived at IVIA, I was not feeling confident of my skills as a researcher, and you gave me the opportunity to develop my career. I will be forever grateful for that. Marcel, you are a special person and you inspired me in many different ways. I admire your ability of multi-tasking, your leadership skills and how you disseminate science (even your insect T-shirts in important talks are an example to me!). You have been far away from IVIA, but it seemed that you were in an office next to mine because you have been highly involved during my whole thesis project. It is incredible how much value you have added to this project, which would have been completely different without your supervision. Thank you. 
I would like to thank other important people involved in this project. Joel, you have been in charge of all the things that we, as entomologists and ecologists, did not master. With your methodology for the chemical analysis, you have succeeded in detecting a few particles of insecticides in billion parts of honeydew! You have been motivated during the whole project and again, you have been a main player in this project. Alberto, you have always given me credits and made me feel confident of myself. Thanks for being there when I needed to, as a scientist and as a colleague! George and Jonathan, my experience in Minnesota was a great success because of you. Because of your efforts in planning and organizing everything before and during my stay. With the rest of the team, we have discovered something highly relevant for nature, science and society. Thanks for your help; I must say that my stay was perfect because of you. The technicians Miquel, Mariano, Jose, and Ana thanks for your help and suggestions during the experiments, and for making IVIA the way it is. Finally, Yolanda, Daniele and Pau, thanks for helping us with the chemical analysis.

Al departamento de E.T.S.I. Agrónomos de Madrid, por haberme enseñado mi pasión por los insectos. Creo que no habría llegado hasta aquí sin haberos conocido. Sois pasión, bondad y profesionalidad. A ti especialmente, Ángeles, por haberme pasado la información sobre la beca, gracias a la cual he cosechado todos estos frutos. Y a ti Celeste, por todo el intercambio de información que hemos tenido a lo largo de todos estos años. También estoy muy agradecido a la gente del IVIA. A mis compañer@s de despachos pre doctorales o zulo: Ruth, te deseo lo mejor, me enseñaste muchísimo cuando llegué al IVIA. Sarra, te admiro por todo lo que has conseguido ante cualquier adversidad. Eres una guerrera. Milena, mi "hermanis": para seguir siendo hermanos, ¡tienes que llevarme a Costa Rica! Me encanta tu energía y positivismo. Angeliki, creo que Margarita ha sido el primer bebe por el que me he emocionado en mi vida. Supongo que es porque sé lo feliz que va a ser contigo, ¡madraza! Alice, you are just wonderful. Thanks for all your help with my English and for being my vegetarian support at IVIA! More importantly, don't leave Science, it needs you! Marta, no tengo palabras para describir la gran persona que eres. Eres la Gandhi del IVIA. No cambies. Jesica, cada día echo de menos llorar juntos sobre la tesis y la vida. Mi compa de lloros, te deseo lo mejor. Ángel, gran entomólogo y mejor persona. Eres increíble, me encanta tener debates de ciencia y fútbol contigo. Solo te falta un corazón colchonero. Muchísimas gracias por las fotos de la tesis, sin ti, sería una tesis mucho menos apetecible y aburrida. A otros compañeros que están o han estado en algún momento u otro en el IVIA, y que su ayuda ha sido también importante. Angelos, por enseñarme tanto de entomología y por ser mi apoyo en tantos momentos. Mi paraninfo y mi gran amigo. Maite, mi paloma mensajera de Wageningen, jte vas a 
convertir en la mejor profe de biología de Valencia!, gracias por tu apoyo. Makrina y Lucia, mis grandes motivadoras para convivir en una sociedad con un modo de vida normalmente distinto al nuestro. Martina y Elena, sois las mejores estadistas que la ciencia ha parido! Gracias por vuestra ayuda. Pablo, ¿por qué has llegado tan tarde al IVIA y no el primer día que llegué? Carlos, por esos debates mesetariano-valencianos que tenemos a diario. Terence, I have no words to describe the great person you are! I miss you since the day you left. Meritxell, muchísimas gracias por tus apoyos, consejos de vida y de carrera. Paco, haces del pasillo de abajo un lugar cálido y agradable; se agradece, y mucho. Cesar, si alguna vez dejas la ciencia, te quiero ver como presentador del tiempo en la televisión. Omar, Carolina, Chaymaa, Edu, Paula, David, Josep, Rachele, Delia, Diego, David, Michelle, Carl, Marian, Dora, Almu, David, Leo, Elena, Mamen, Maria José, Raúl, Azu, María, Miriam, Julieta, Tamara, todo el personal del IVIA, y todas las personas que me haya olvidado, gracias por vuestra ayuda, charlas, tartas, o simplemente, por haberme hecho sonreír durante el doctorado. Os deseo lo mejor. También me gustaría agradecer a la cafetería. ¡Sois las mejores! El pensar en el bocata que tomaré durante el almuerzo me ayuda a levantarme a diario. Finally, I would like to thank my loyal squire, the manual counter, for being present always when I needed it. You allowed me to count more efficiently 184,791 droplets of honeydew throughout my thesis project! Thank you so much.

A mis amigos de Madrid. A los del cole: Nuria, mi divulgadora de tesis. Gracias por tu ayuda. A Jaime, el mejor artista del mundo, gracias por todos tus diseños de tesis y por tu apoyo general. A los doctores Pilin, Parra y Pablo M., por las charlas científicas que hemos tenido a lo largo de estos años. A Juan, mi apoyo colchonero y gran camarenero. A Diego, Wini, Explorador, Luigi, Pablo A., Cris, Titeon, Elena, Felipe, White, Álvaro, Eva y Bea, Alcu, Peps, Lozac, Danilo, Pastor, Marina, y tod@s los demás amig@s que me he dejado. La lista es infinita pero no excluyente. Muchas gracias a tod@s por hacerme sentir en casa cuando voy a Madrid. No os imagináis cuanta fuerza me ha dado teneros cerca durante estos 4 años.

A los agrónomos, gracias por todas las risas y locuras, por hacer de la carrera los años más locos de mi vida y por hacer que a un urbanita le guste la agronomía. Creo que fue por el buen rollo que tuvimos. Hemos aprendido, destruido, bebido, bailado, llorado, estudiado, compartido, y sobretodo, reído. Ana, Miguel, Rodri, Erni, Luci, Esterete, Maite, Nugget, Marcos, Sego, Castaño, Pacs, Helena, Belén, Dudu, Tere, Jessica, gracias de corazón a tod@s. A Lisanne, Lucas, Soto, y demás gente de la Asociación Internacional de Estudiantes de Agricultura, por hacerme disfrutar de la carrera y 
complementarla con una de las mejores experiencias que he vivido, os quiero. Estáis tan lejos y sin embargo os siento tan cerca.

A mis compañeros de Master de Wageningen, a Catania: Claves, Mae, Jose, Pau, Lord y Fre. For those parties, nights playing Catan, and meetings that made my Erasmus and Masters at Wageningen an unforgettable experience.

A mi amigo Carlos, a quien le debo haberlo pasado bien en Minnesota y haber podido disfrutar de mi estancia. A Mimmo, Carlos, Elena, Melisa, Aitor, Epi, y Laura, que sois mi familia en Valencia.

A los colegas de la Asociación Valenciana de Apicultura Urbana, y en especial a Raúl, que tantas energías dais y das. Pronto legalizaremos la apicultura urbana en Valencia primero y en España después, y llenaremos Valencia de flores para que sea un lugar con mucha más biodiversidad de polinizadores. Ojalá podamos realizar todos los proyectos que tenemos entre manos. ¡Nos queda poco! A la gente de mi huerto urbano, y en especial a Juan, que tanto te lo curras. No sabes lo bien que me ha venido el huerto para desconectar de la tesis, y en general, de los agobios cotidianos que me han ido surgiendo. Gracias.

Alla mia famiglia italiana. Siete tanti che non voglio scrivere tutti nomi perché sicuro dimentico a qualcuno, ma vi ringrazio a tutti. Nos sapete quanto ho imparato da vuoi, della vostra coltura, cibo, storia, lingua, modo di vita e soprattutto, per farmi scoprire le vere pizze. Sono una persona molto più felice grazie a voi.

A mi familia que tanto quiero. A mi abuela, que poco a poco pierde su cabeza, pero siempre recuerda cuanto me acariciaba la espalda cuando era pequeño. A mi abuelo, que seguramente esté jugando una partida de cartas allá donde esté, y apoyándome desde lo más lejano. A mi madre, que además de ser la mejor madre del mundo, creo que no conozco una persona tan buena y generosa como tú. Gracias por tu apoyo, por querernos tanto y preocuparte por nosotros. A mi padre, que tanto me ha apoyado con todas las decisiones de mi vida. Me apoyas todos los días, con "charletas", mensajes, cartas, o por teléfono, y yo lo agradezco infinitamente. A los dos, que me habéis mimado tanto, me habéis dado todas las oportunidades que he querido para formarme, viajar, aprender, crecer como persona y estudiar lo que he querido. No dudéis ni un segundo que todo lo que he hecho es fruto de vuestro apoyo, animo, educación y amor. A mi hermano, que tanto me apoyas, me haces reír, festejar, me ayudas a creer en lo que hago, y me enseñas a valorarme. Eres generoso, buena persona, honesta y el mejor hermano que uno 
puede pedir. A mi cuñada Carol, no conozco un mejor match para Gabo. Me encanta lo enérgica, buena persona y positiva que eres. A Kobe, y a Gin, mis dos soles, que me dais alegría cada segundo que os veo o acaricio. A Río, que inesperadamente te has ido tan pronto de la familia, pero que de alguna manera sigues estando con nosotros.

A Lorenza. Que no sé ni por dónde empezar, porque entonces no acabo. Eres todo, eres mi energía, el azúcar de mi vida, porque derrochas felicidad, apoyo, amor, mucho amor, locura, mucha locura, eres fuente de conocimiento y un laboratorio de ideas, eres mi gran motivadora, una persona con muchos valores, bondadosa, tus consejos no sé cómo lo haces, pero son oro para mí, y no sé cómo decirte que eres la mejor compañera de vida que uno puede tener y que te quiero tanto. 

About the author
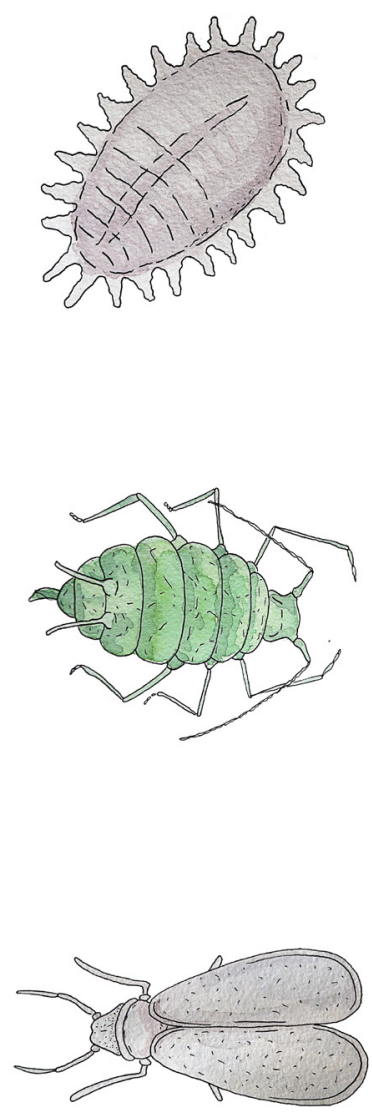

Miguel Calvo Agudo was born on July $5^{\text {th }}, 1991$ in Madrid, Spain. He studied agricultural engineering in a 5-year program at the Polytechnic University in Madrid. During his studies, he became the National Director of the International Association of Agricultural Students and related sciences (IAAS), where he organized and participated in numerous congresses and seminars worldwide. During his last year, he went on an Erasmus exchange to Wageningen University (WUR) in The Netherlands. He liked WUR so much that he decided to do a 2-year Masters in Plant Science there. He carried out the research for his MSc Thesis at Biobest Belgium, one of the most important companies on biological control and pollination. There, he studied how to increase hive productivity and flight activity of the bumblebee Bombus terrestris using pheromones. For his internship, he moved to the Instituto Valenciano de Investigaciones Agrarias (IVIA) in Valencia, Spain, where he studied whether honeydew is a route of insecticide exposure to beneficial insects. The results were so promising that he decided to continue his investigations for his $\mathrm{PhD}$, which was done at IVIA and WUR.

His PhD project was supervised by Dr. Alejandro Tena and Prof. Dr. Marcel Dicke. He discovered an important route of exposure to insecticides for beneficial insects that was published in the prestigious journal Proceedings of the Natural Academy of Sciences of the USA (PNAS). During his $\mathrm{PhD}$, he moved to the University of Minnesota (U.S.A.) to work with Prof. Dr. George E. Heimpel on the negative impacts of seeds treated with insecticides on biological control agents. During his last year of $\mathrm{PhD}$, he also wrote a perspective manuscript to openthis area of research in the field of ecotoxicology to other research institutes and environmental protection agencies. The last year of his $\mathrm{PhD}$, he started teaching gardeners at the Escuela de Jardins de Valencia (OAM-Escuela de Jardinería) in different courses focused on biodiversity and control of pests in gardens.

Miguel is a board member of the Urban Apiculture Association in Valencia (AVAU), which aims to legalize apiculture in Spanish cities, and works together with the local government to make the city of València a sanctuary for all pollinators. In his spare time, Miguel likes to work on his urban allotment where he grows sustainably the vegetables he eats all year round.

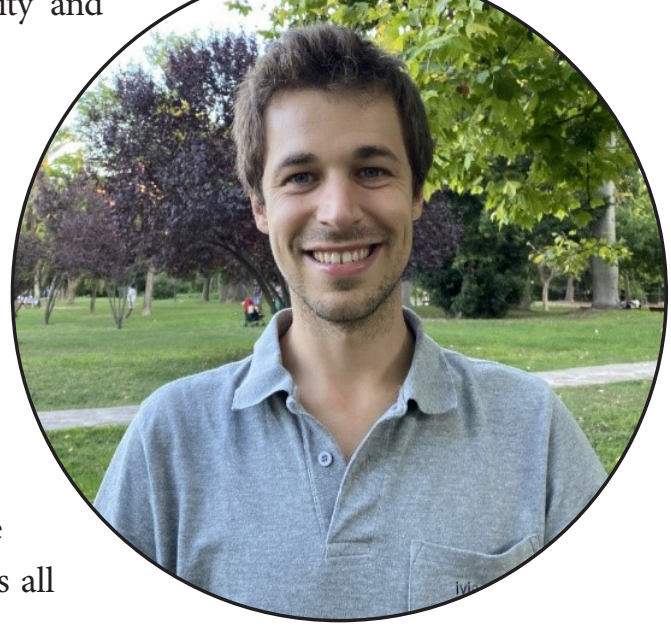



List of publications by Miguel Calvo-Agudo
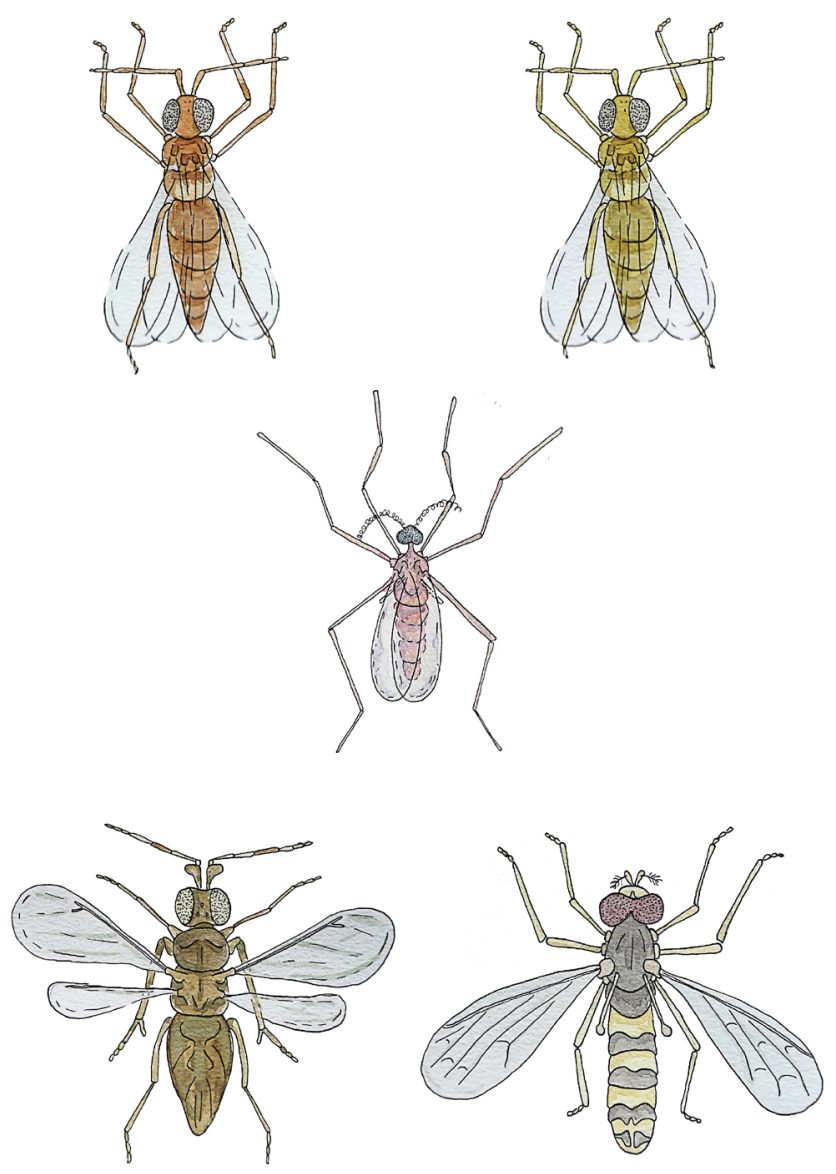



\section{LIST OF PUBLICATIONS BY MIGUEL CALVO-AGUDO}

\section{Published papers}

Calvo-Agudo, M., González-Cabrera, J., Picó, Y., Calatayud-Vernich, P., Urbaneja, A., Dicke, M., \& Tena, A. (2019). Neonicotinoids in excretion product of phloem-feeding insects kill beneficial insects. Proceedings of the National Academy of Sciences of the U.S.A., 116(34), 16817-16822.

Calvo-Agudo, M., González-Cabrera, J., Sadutto, D., Picó, Y., Urbaneja, A., Dicke, M., \& Tena, A. (2020). IPM-recommended insecticides harm beneficial insects through contaminated honeydew. Environmental Pollution, 267, 115581.

Calvo-Agudo, M., Dregni, J., González-Cabrera, J., Dicke, M., Heimpel, GE., \& Tena, A. (2021). Neonicotinoids from coated seeds toxic for honeydew-feeding biological control agents. Environmental Pollution, 289, 117813.

\section{In preparation and submitted}

Calvo-Agudo, M., Tooker, J.F., Dicke, M., \& Tena, A. (2021). Insecticide-contaminated honeydew: risks for beneficial insects in agriculture. Submitted.

Calvo-Agudo, M., González-Cabrera, J., Dicke, M., \& Tena, A. (2021). Beneficial insects do not avoid feeding on honeydew contaminated with neonicotinoids and may even prefer feeding on it. 
This research was conducted under the auspices of the Instituto Valenciano de Investigaciones Agrarias (IVIA, Spain) (Project RTA2017-00095) and granted by Instituto Nacional de Investigaciones Agrarias (INIA, Spain) (CPD2016-0085) and "Europa Excelencia" project (EUR2020-112293).

Cover illustrations by Ángel Plata Sánchez

Cover design and thesis layout by Miguel Calvo Agudo and Jaime Castilla Santos

Typesetting edition by Blanca Moncunill Solé

Printed by GVO drukkers en vormgevers B.V., Ede, The Netherlands 


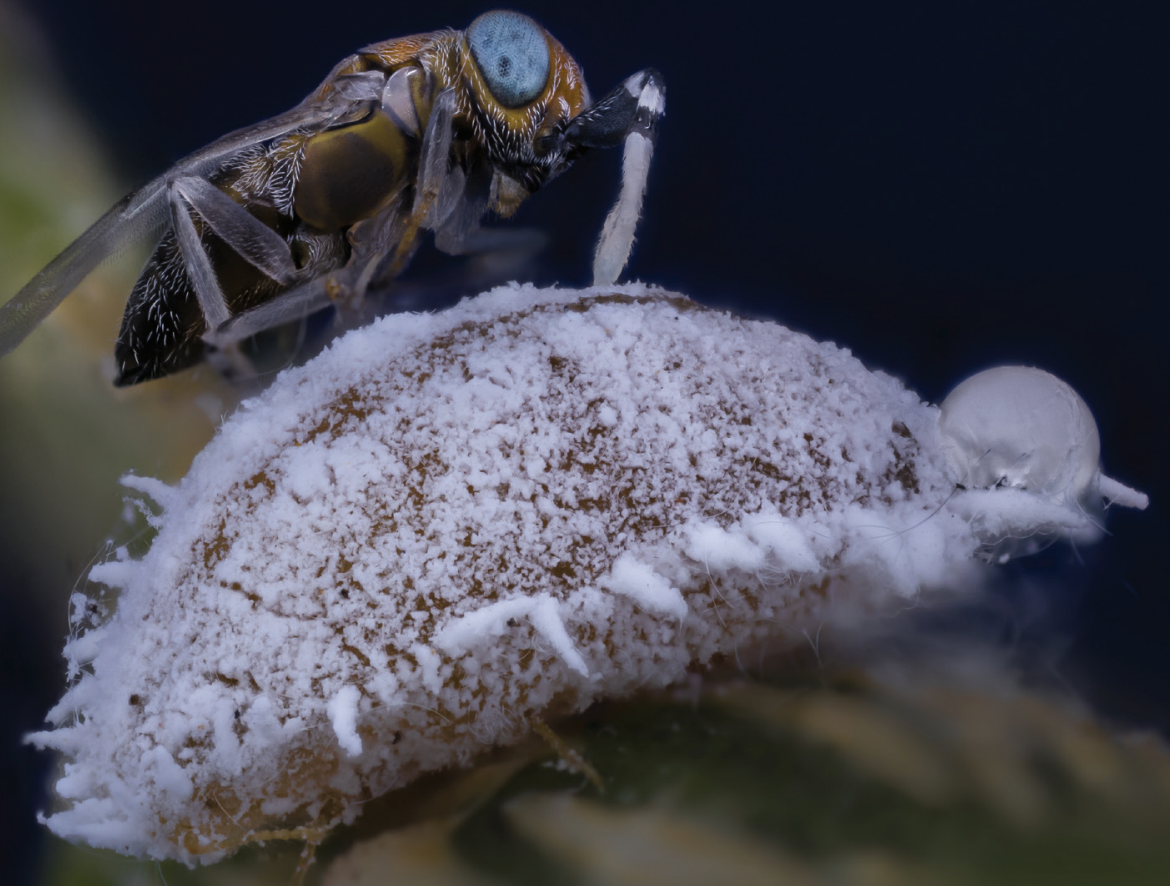

